\title{
Supplemntary Materials
}

\section{Enantioselective [4+2] Cycloaddition Reaction of Vinylquinolines with Dienals Enabled by Synergistic Organocatalysis}

\author{
Jing Chen, ${ }^{\dagger,+}, \#$ Yiwei Fu, ${ }^{\dagger, \#}$ Yang Yu, Jian-Rong Wang, ${ }^{\dagger}$ Yue-Wei Guo, ${ }^{\dagger *}$ Hao Li $^{\dagger *}$ and Wei \\ Wang ${ }^{\dagger}, *$ \\ State Key Laboratory of Bioengineering Reactor, Shanghai Key Laboratory of New Drug \\ Design, and School of Pharmacy, East China University of Science and Technology, 130 \\ Meilong Road, Shanghai 200237, China \\ ${ }^{7}$ State Key Laboratory of Drug Research, Shanghai Institute of Materia Medica, Chinese \\ Academy of Sciences, 555 Zuchongzhi Road, Shanghai 201203, China \\ §Departments of Pharmacology \& Toxicology and Chemistry \& Biochemistry University of \\ Arizona, Tucson, AZ 85721, USA
}

\section{Table of Contents}

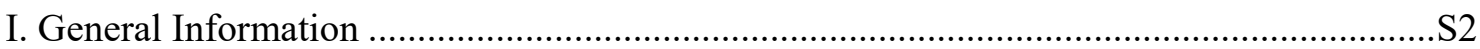

II. General procedure for the preparation of vinylquinoline and characterization. ................S3

III. General Procedure for Asymmetric Formal [4+2] cycloaddition reactions and

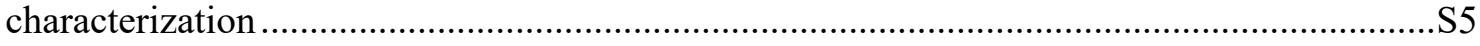

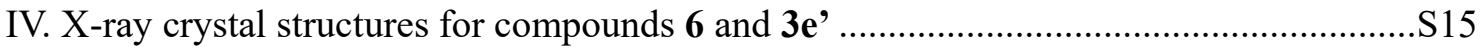

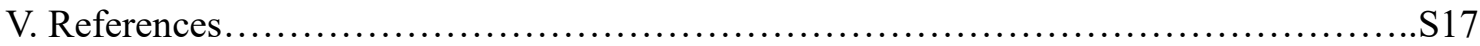

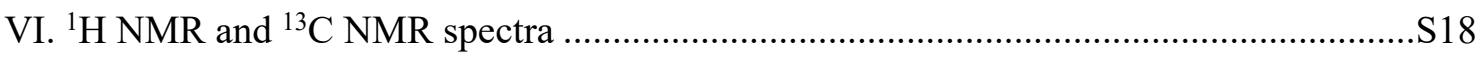

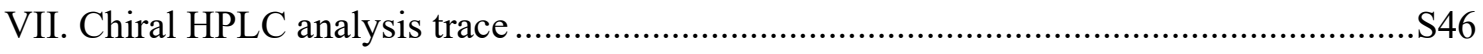


I. General Information: Commercial reagents were used as received, unless otherwise stated. ${ }^{1} \mathrm{H}$ and ${ }^{13} \mathrm{C}$ NMR spectra were recorded on Bruke DRX 400 (400 MHz), and tetramethylsilane (TMS) was used as a reference. Data for ${ }^{1} \mathrm{H}$ are reported as follows: chemical shift (ppm), and multiplicity $(\mathrm{s}=$ singlet, $\mathrm{bs}=$ broad singlet, $\mathrm{d}=$ doublet, $\mathrm{t}=$ triplet, $\mathrm{q}=$ quartet, $\mathrm{m}=$ multiplet $)$. Data for ${ }^{13} \mathrm{C}$ NMR are reported as ppm. Chromatography was carried out with silica gel (200$300 \mathrm{mesh}$ ) using mixtures of petroleum ether (b.p. $60-80^{\circ} \mathrm{C}$ ) and ethyl acetate as eluents. The enantiomeric excess of products was detected on HPLC (Shimadzu LC-LabSolutions). Mass Spectra were obtained from East China University of Science and Technology mass spectral facility. 


\section{General procedure for the preparation of vinylquinoline and characterization.}

Vinylquinoline derivatives were synthesized via suzuki cross-coupling reaction ${ }^{1}$ : To a solution of 4-haloquinolines (5.0 mmol), $\mathrm{Pd}\left(\mathrm{Ph}_{3} \mathrm{P}\right)_{2} \mathrm{Cl}_{2}(0.5 \mathrm{mmol})$ and $\mathrm{CsF}(15 \mathrm{mmol})$ in dioxane $(10$ $\mathrm{mL})$ and $\mathrm{H}_{2} \mathrm{O}(5 \mathrm{~mL})$ was added 4,4,5,5-tetramethyl-2-vinyl-1,3,2-dioxaborolane (1.5 $\left.\mathrm{mL}\right)$. The mixture was stirred for 12 hours at $85{ }^{\circ} \mathrm{C}$ in oil bath under $\mathrm{N}_{2}$ atmosphere. Then the suspension was cooled to room temperature and extract with EtOAc $(15 \mathrm{~mL})$. Dried by $\mathrm{MgSO}_{4}$, concentrated, the crude product was purified by a short column chromatography (PE / EA) on silica gel to give 4-vinylquinoline derivatives.

4-Vinylquinoline and 6-methoxy-4-vinylquinoline was synthesized following the procedure in reference $2 .^{2}$

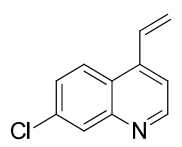

7-Chloro-4-vinylquinoline The title compound was prepared according to the general procedure using 4,7-dichloroquinoline as the starting material in $71 \%$ yield $(700 \mathrm{mg})$ as a brown solid. ${ }^{1} \mathrm{H}$ NMR $\left(\mathrm{CDCl}_{3}, 400 \mathrm{MHz}\right): \delta 8.87-8.86(\mathrm{~d}, J=4.4 \mathrm{~Hz}, 1 \mathrm{H}), 8.12(\mathrm{~s}, 1 \mathrm{H}), 8.04-$ $8.02(\mathrm{~d}, J=8.9 \mathrm{~Hz}, 1 \mathrm{H}), 7.52-7.50(\mathrm{~d}, J=8.9 \mathrm{~Hz}, 1 \mathrm{H}), 7.46-7.45(\mathrm{~d}, J=4.4 \mathrm{~Hz}, 1 \mathrm{H}), 7.40-7.33$ $\left(\mathrm{dd}, J_{l}=17.3 \mathrm{~Hz}, J_{2}=11.0 \mathrm{~Hz}, 1 \mathrm{H}\right), 6.01-5.97(\mathrm{~d}, J=17.3 \mathrm{~Hz}, 1 \mathrm{H}), 5.71-5.68(\mathrm{~d}, J=11.0 \mathrm{~Hz}$, $1 \mathrm{H}) ;{ }^{13} \mathrm{C} \mathrm{NMR}\left(\mathrm{CDCl}_{3}, 100 \mathrm{MHz}\right): \delta 151.2,148.8,143.7,135.3,131.6,128.8,127.6,125.0$, 124.6, 121.5, 117.6; HRMS (EI) m/z: Calcd for $\mathrm{C}_{11} \mathrm{H}_{8} \mathrm{ClN}$ 189.0345; Found 189.0347.

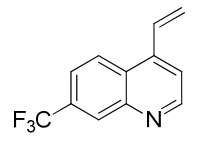

7-(Trifluoromethyl)-4-vinylquinoline The title compound was prepared according to the general procedure using 4-bromo-7-(trifluoromethyl)quinoline as the starting material in 78\% yield $(870 \mathrm{mg})$ as a white solid. ${ }^{1} \mathrm{H} \mathrm{NMR}\left(\mathrm{CDCl}_{3}, 400 \mathrm{MHz}\right): \delta 8.98-8.97 \quad(\mathrm{~d}, J=4.5 \mathrm{~Hz}$, $1 \mathrm{H}), 8.43(\mathrm{~s}, 1 \mathrm{H}), 8.23-8.21(\mathrm{~d}, J=8.8 \mathrm{~Hz}, 1 \mathrm{H}), 7.75-7.73(\mathrm{~d}, J=8.8 \mathrm{~Hz}, 1 \mathrm{H}), 7.58-7.57(\mathrm{~d}$, $J=4.5 \mathrm{~Hz}, 1 \mathrm{H}), 7.45-7.37\left(\mathrm{dd}, J_{l}=17.3 \mathrm{~Hz}, J_{2}=11.0 \mathrm{~Hz}, 1 \mathrm{H}\right), 6.05-6.01(\mathrm{~d}, J=17.3 \mathrm{~Hz}, 1 \mathrm{H})$, 5.76-5.73 (d, $J=11.0 \mathrm{~Hz}, 1 \mathrm{H}) ;{ }^{13} \mathrm{C} \mathrm{NMR}\left(\mathrm{CDCl}_{3}, 100 \mathrm{MHz}\right): \delta 151.4,151.0,147.3,143.8$, $131.3,131.2,127.7,125.1,125.0,122.5,122.4,122.4,122.4,122.2,122.2,122.0,119.1$. HRMS (EI) m/z calcd for $\mathrm{C}_{9} \mathrm{H}_{6} \mathrm{~F}_{3} \mathrm{NO}_{3}$ (M-1) 223.0609, found 223.0610.

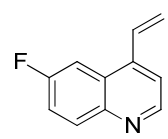

6-Fluoro-4-vinylquinoline The title compound was prepared according to the general procedure using 4-chloro-6-fluoroquinoline as the starting material in $75 \%$ yield $(649 \mathrm{mg})$ as white solid. ${ }^{1} \mathrm{H}$ NMR $\left(\mathrm{CDCl}_{3}, 400 \mathrm{MHz}\right): \delta 8.80-8.79(\mathrm{~d}, J=4.5 \mathrm{~Hz}, 1 \mathrm{H}), 8.09-8.05\left(\mathrm{dd}, J_{1}=\right.$ $\left.9.2 \mathrm{~Hz}, J_{2}=5.6 \mathrm{~Hz}, 1 \mathrm{H}\right), 7.65-7.62\left(\mathrm{dd}, J_{1}=10.2 \mathrm{~Hz}, J_{2}=2.8 \mathrm{~Hz}, 1 \mathrm{H}\right), 7.47-7.42(\mathrm{~m}, 2 \mathrm{H})$, 7.27-7.20 (dd, $\left.J_{1}=17.3 \mathrm{~Hz}, J_{2}=11.0 \mathrm{~Hz}, 1 \mathrm{H}\right), 5.96-5.91(\mathrm{~d}, J=17.3 \mathrm{~Hz}, 1 \mathrm{H}), 5.65-5.62(\mathrm{~d}, J$ 
$=11.0 \mathrm{~Hz}, 1 \mathrm{H}) ;{ }^{13} \mathrm{C} \mathrm{NMR}\left(\mathrm{CDCl}_{3}, 100 \mathrm{MHz}\right): \delta 161.6,159.2,149.4,149.4,145.6,142.9,142.8$, 132.4, 132.3, 131.5, 126.8, 126.8, 121.0, 119.5, 119.3, 117.8, 107.1, 107.0; HRMS (EI) m/z: Calcd for $\mathrm{C}_{11} \mathrm{H}_{8} \mathrm{FN}$ 173.0641; Found 173.0640.

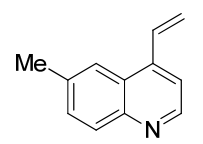

6-Methyl-4-vinylquinoline The title compound was prepared according to the general procedure using 4-chloro-6-methylquinoline as the starting material in $81 \%$ yield (684 $\mathrm{mg}$ ) as brown solid. ${ }^{1} \mathrm{H}$ NMR $\left(\mathrm{CDCl}_{3}, 400 \mathrm{MHz}\right): \delta 8.80-8.79(\mathrm{~d}, J=4.6 \mathrm{~Hz}, 1 \mathrm{H}), 8.01-7.99(\mathrm{~d}, J=$ $8.6 \mathrm{~Hz}, 1 \mathrm{H}), 7.83(\mathrm{~s}, 1 \mathrm{H}), 7.54-7.52\left(\mathrm{dd}, J_{1}=8.5 \mathrm{~Hz}, J_{2}=1.7 \mathrm{~Hz}, 1 \mathrm{H}\right), 7.44-7.37(\mathrm{~m}, 2 \mathrm{H}), 2.54$ $(\mathrm{s}, 3 \mathrm{H}) ;{ }^{13} \mathrm{C} \mathrm{NMR}\left(\mathrm{CDCl}_{3}, 100 \mathrm{MHz}\right): \delta 149.3,147.0,142.7,136.4,132.2,131.4,129.6,126.1$, 122.4, 120.3, 117.4, 21.9; HRMS (EI) m/z: Calcd for $\mathrm{C}_{12} \mathrm{H}_{11} \mathrm{~N}$ 169.0891; Found 169.0890.

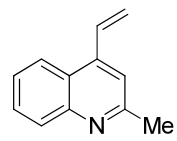

2-Methyl-4-vinylquinoline The title compound was prepared according to the general procedure using 4-chloro-2-methylquinoline as the starting material in $65 \%$ yield $(550 \mathrm{mg})$ as a white solid. ${ }^{1} \mathrm{H}$ NMR $\left(\mathrm{CDCl}_{3}, 400 \mathrm{MHz}\right): \delta 8.04-8.02(\mathrm{~d}, J=8.7 \mathrm{~Hz}, 2 \mathrm{H}), 7.69-7.65(\mathrm{~m}, 1 \mathrm{H})$, 7.51-7.47 (m, 1H), 7.42-7.35 (dd, $\left.J_{l}=17.3 \mathrm{~Hz}, J_{2}=11.0 \mathrm{~Hz}, 1 \mathrm{H}\right), 7.35$ (s, 1H), 5.97-5.93 (dd, $\left.J_{l}=17.3 \mathrm{~Hz}, J_{2}=1.0 \mathrm{~Hz}, 1 \mathrm{H}\right), 5.64-5.61\left(\mathrm{dd}, J_{1}=11.0 \mathrm{~Hz}, J_{2}=1.0 \mathrm{~Hz}, 1 \mathrm{H}\right), 2.74(\mathrm{~s}, 3 \mathrm{H}) ;{ }^{13} \mathrm{C}$ NMR (CDCl3, $100 \mathrm{MHz}$ ): $\delta$ 158.7, 148.1, 143.5, 132.2, 129.2, 129.1, 125.6, 124.5, 123.3, 120.3, 118.2, 25.3; HRMS (EI) m/z: Calcd for $\mathrm{C}_{12} \mathrm{H}_{11} \mathrm{~N}$ 169.0891; Found 169.0889.

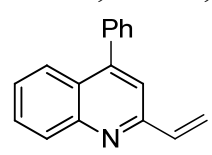

4-Phenyl-2-vinylquinoline The title compound was prepared according to the general procedure using 4-chloro-6-methylquinoline as the starting material in $81 \%$ yield $(936 \mathrm{mg})$ as a white solid. ${ }^{1} \mathrm{H} \mathrm{NMR}\left(\mathrm{CDCl}_{3}, 400 \mathrm{MHz}\right): \delta 8.15-8.13(\mathrm{~d}, J=8.4 \mathrm{~Hz}, 1 \mathrm{H}), 7.87-7.85$ (dd, $\left.J_{l}=0.9 \mathrm{~Hz}, J_{2}=8.4 \mathrm{~Hz}, 1 \mathrm{H}\right), 7.72-7.68\left(\mathrm{ddd}, J_{1}=1.4 \mathrm{~Hz}, J_{2}=6.9 \mathrm{~Hz}, J_{3}=8.4 \mathrm{~Hz}, 1 \mathrm{H}\right), 7.56-$ $7.49(\mathrm{~m}, 6 \mathrm{H}), 7.47-7.43\left(\mathrm{ddd}, J_{1}=1.2 \mathrm{~Hz}, J_{2}=6.99 \mathrm{~Hz}, J_{3}=8.2 \mathrm{~Hz}, 1 \mathrm{H}\right), 7.11-7.04\left(\mathrm{dd}, J_{l}=\right.$ $\left.11.0 \mathrm{~Hz}, J_{2}=17.7 \mathrm{~Hz}, 1 \mathrm{H}\right), 6.32-6.28(\mathrm{~d}, J=17.7 \mathrm{~Hz}, 1 \mathrm{H}), 5.70-5.67(\mathrm{~d}, J=11.0 \mathrm{~Hz}, 1 \mathrm{H}) ;{ }^{13} \mathrm{C}$ NMR (CDCl $3,100 \mathrm{MHz}): \delta 155.5,148.7,148.5,138.2,137.9,129.7,129.5,129.4,128.5,128.4$, 126.3, 125.6, 119.9, 118.6; HRMS (EI) m/z: Calcd for $\mathrm{C}_{17} \mathrm{H}_{13} \mathrm{~N} 231.1048$; Found 231.1046. 
III. General Procedure for Asymmetric Formal [4+2] cycloaddition reactions and characterization (3a as example): To a solution of 4-vinylquinoline 1a (31 mg, $0.2 \mathrm{mmol}$ ) and 4-phenylhexa-2,4-dienal $\mathbf{2 a}(69 \mathrm{mg}, 0.4 \mathrm{mmol})$ in $\mathrm{CH}_{2} \mathrm{Cl}_{2}(0.8 \mathrm{~mL})$ was added catalyst $\mathbf{I}$ (13 mg, $0.04 \mathrm{mmol})$ and $\mathrm{CH}_{3} \mathrm{SO}_{3} \mathrm{H}(9.6 \mathrm{mg}, 0.1 \mathrm{mmol})$. The resulting solution was stirred for $72 \mathrm{~h}$ at room temperature. The crude reaction mixture was directly purified by column chromatography on silica gel $(\mathrm{PE}: \mathrm{EA}=1: 2)$ to give the corresponding product $27 \mathrm{mg}$ in total $94 \%$ yield $\left(\mathbf{3 a}: 3 \mathbf{a}^{\prime}=42: 52\right)$.<smiles>O=CC[C@@H]1C(c2ccnc3ccccc23)=CCCC1c1ccccc1</smiles>

2-((2R,3S)-3-(Quinolin-4-yl)-2,3,4,5-tetrahydro-[1,1'-biphenyl]-2-yl)acetaldehyde (3a): The title compound was prepared according to the general procedure, as described above in $42 \%$ yield $(27 \mathrm{mg})$ as yellow oil. ${ }^{1} \mathrm{H} \mathrm{NMR}\left(\mathrm{CDCl}_{3}, 400 \mathrm{MHz}\right): \delta 9.57-9.56(\mathrm{t}, J=1.3 \mathrm{~Hz}, 1 \mathrm{H})$, $8.85-8.84(\mathrm{~d}, J=4.6 \mathrm{~Hz}, 1 \mathrm{H}), 8.19-8.17(\mathrm{~d}, J=8.3 \mathrm{~Hz}, 1 \mathrm{H}), 8.10-8.08(\mathrm{~d}, J=8.4 \mathrm{~Hz}, 1 \mathrm{H})$, $7.75-7.71\left(\mathrm{dt}, J_{I}=7.0 \mathrm{~Hz}, J_{2}=1.1 \mathrm{~Hz}, 1 \mathrm{H}\right), 7.62-7.58\left(\mathrm{dt}, J_{l}=7.0 \mathrm{~Hz}, J_{2}=1.1 \mathrm{~Hz}, 1 \mathrm{H}\right), 7.45-$ $7.36(\mathrm{~m}, 5 \mathrm{H}), 7.33-7.31(\mathrm{~m}, 1 \mathrm{H}), 6.13(\mathrm{~s}, 1 \mathrm{H}), 3.84-3.83(\mathrm{~m}, 1 \mathrm{H}), 3.73-3.72(\mathrm{~m}, 1 \mathrm{H}), 2.66-2.59$ $\left(\mathrm{ddd}, J_{1}=17.7 \mathrm{~Hz}, J_{2}=8.3 \mathrm{~Hz}, J_{3}=1.8 \mathrm{~Hz}, 1 \mathrm{H}\right), 2.52-2.47\left(\mathrm{ddd}, J_{1}=17.7 \mathrm{~Hz}, J_{2}=3.2 \mathrm{~Hz}, J_{3}\right.$ $=1.1 \mathrm{~Hz}, 1 \mathrm{H}), 2.30-2.25(\mathrm{~m}, 1 \mathrm{H}), 2.07-1.96(\mathrm{~m}, 1 \mathrm{H}) ;{ }^{13} \mathrm{C} \mathrm{NMR}\left(\mathrm{CDCl}_{3}, 100 \mathrm{MHz}\right): \delta 201.9$, $150.8,149.9,148.1,141.1,139.4,130.3,129.2,128.8,127.9,127.5,127.0,126.7,126.3,122.9$, 118.6, 47.6, 38.0, 35.7, 25.4, 23.5; HRMS (EI) m/z: Calcd for $\mathrm{C}_{23} \mathrm{H}_{21} \mathrm{NO}$ 327.1623; Found 327.1619; HPLC (Chiralpak AD-3, $i-\mathrm{PrOH} / \mathrm{h}$ exane $=20 / 80$, flow rate $=1.0 \mathrm{~mL} / \mathrm{min}, \lambda=254$ $\mathrm{nm}): \mathrm{t}_{\text {minor }}=11.6, \mathrm{t}_{\text {major }}=10.7$, ee $=98 \%$.<smiles>O=CC[C@H]1C(c2ccccc2)=CCC[C@H]1c1ccnc2ccccc12</smiles>

2-((2R,3R)-3-(Quinolin-4-yl)-2,3,4,5-tetrahydro-[1,1'-biphenyl]-2-yl)acetaldehyde (3a'): The title compound was prepared according to the general procedure, as described above in $52 \%$ yield $(34 \mathrm{mg})$ as yellow oil. ${ }^{1} \mathrm{H} \mathrm{NMR}\left(\mathrm{CDCl}_{3}, 400 \mathrm{MHz}\right): \delta 9.77(\mathrm{~s}, 1 \mathrm{H}), 8.66(\mathrm{~d}, J=2.4$ $\mathrm{Hz}, 1 \mathrm{H}), 8.45(\mathrm{~d}, J=2.4 \mathrm{~Hz}, 1 \mathrm{H}), 7.64(\mathrm{~s}, 1 \mathrm{H}), 7.13-7.16(\mathrm{~m}, 1 \mathrm{H}), 6.85(\mathrm{~d}, J=8.0,1 \mathrm{H}), 6.66-$ $6.70(\mathrm{~m}, 2 \mathrm{H}), 4.77\left(\mathrm{dd}, J_{l}=2.0 \mathrm{~Hz}, J_{2}=8.8 \mathrm{~Hz}, 1 \mathrm{H}\right), 3.81(\mathrm{~s}, 3 \mathrm{H}), 3.69\left(\mathrm{dd}, J_{l}=2.0 \mathrm{~Hz}, J_{2}=\right.$ $18.4 \mathrm{~Hz}, 1 \mathrm{H}), 3.39$ (dd, $\left.J_{1}=8.8 \mathrm{~Hz}, J_{2}=18.4 \mathrm{~Hz}, 1 \mathrm{H}\right) ;{ }^{13} \mathrm{C} \mathrm{NMR}\left(\mathrm{CDCl}_{3}, 100 \mathrm{MHz}\right): \delta 199.4$, 150.0, 149.4, 147.6, 140.6, 140.4, 138.7, 130.2, 129.6, 128.6, 127.6, 127.4, 127.2, 126.3, 126.3, 122.9, 119.0, 44.6, 38.7, 35.6, 26.5, 22.1; HRMS (EI) m/z: Calcd for $\mathrm{C}_{23} \mathrm{H}_{21} \mathrm{NO} 327.1623$; Found 327.1632; HPLC (Chiralpak AD-3, $i$-PrOH $/$ hexane $=20 / 80$, flow rate $=1.0 \mathrm{~mL} / \mathrm{min}, \lambda$ $=254 \mathrm{~nm}): \mathrm{t}_{\text {minor }}=8.6, \mathrm{t}_{\text {major }}=11.2$, ee $=95 \%$. Note: Our lab made three attempts to get a pure chromatogram for racemic 3a, unfortunately, only worse chromatograms were obtained. 
<smiles>COc1ccc2nccc(C3CCC=C(c4ccccc4)C3C=O)c2c1</smiles>

2-((2R,3S)-3-(6-Methoxyquinolin-4-yl)-2,3,4,5-tetrahydro-[1,1'-biphenyl]-2-

yl)acetaldehyde (3b): The title compound was prepared according to the general procedure, as described above in $41 \%$ yield $(29 \mathrm{mg})$ as yellow oil. ${ }^{1} \mathrm{H} \mathrm{NMR}\left(\mathrm{CDCl}_{3}, 400 \mathrm{MHz}\right)$ : $\delta$ 9.57$9.56(\mathrm{t}, \mathrm{J}=1.5 \mathrm{~Hz}, 1 \mathrm{H}), 8.70-8.70(\mathrm{~d}, J=3.5 \mathrm{~Hz}, 1 \mathrm{H}), 8.06-8.03(\mathrm{~d}, J=9.1 \mathrm{~Hz}, 1 \mathrm{H}), 7.41-7.35$ $(\mathrm{m}, 6 \mathrm{H}), 7.32-7.29(\mathrm{~m}, 2 \mathrm{H}), 6.12-6.11(\mathrm{t}, J=3.4 \mathrm{~Hz}, 1 \mathrm{H}), 3.96(\mathrm{~s}, 3 \mathrm{H}), 3.75-3.71(\mathrm{~m}, 1 \mathrm{H}), 3.68-$ $3.66(\mathrm{~m}, 1 \mathrm{H}), 2.67-2.60\left(\mathrm{ddd}, J_{l}=1.2 \mathrm{~Hz}, J_{2}=8.0 \mathrm{~Hz}, J_{3}=17.5 \mathrm{~Hz}, 1 \mathrm{H}\right), 2.47-2.42\left(\mathrm{ddd}, J_{l}=\right.$ $\left.1.4 \mathrm{~Hz}, J_{2}=3.2 \mathrm{~Hz}, J_{3}=17.5 \mathrm{~Hz}, 1 \mathrm{H}\right), 2.33-2.26(\mathrm{~m}, 1 \mathrm{H}), 2.07-2.00(\mathrm{~m}, 3 \mathrm{H}) ;{ }^{13} \mathrm{C} \mathrm{NMR}\left(\mathrm{CDCl}_{3}\right.$, $100 \mathrm{MHz}): \delta 201.4,157.9,148.9,147.7,144.5,141.1,139.4,131.9,128.7,127.9,127.4,126.3$, 121.3, 118.9, 101.4, 55.6, 47.2, 38.1, 36.4, 25.3, 23.8; HRMS (EI) m/z: Calcd for $\mathrm{C}_{24} \mathrm{H}_{23} \mathrm{NO}_{2}$ 357.1729, Found 357.1728; HPLC (Chiralpak AD-3, $i$-PrOH/hexane $=20 / 80$, flow rate $=1.0$ $\mathrm{mL} / \mathrm{min}, \lambda=254 \mathrm{~nm}): \mathrm{t}_{\mathrm{minor}}=12.7, \mathrm{t}_{\mathrm{major}}=17.2$, ee $=99 \%$.<smiles>COc1ccc2nccc([C@@H]3CCC=C(c4ccccc4)C3C=O)c2c1</smiles>

2-((2R,3R)-3-(6-Methoxyquinolin-4-yl)-2,3,4,5-tetrahydro-[1,1'-biphenyl]-2-

yl)acetaldehyde (3b'): The title compound was prepared according to the general procedure, as described above in $48 \%$ yield $(34 \mathrm{mg})$ as yellow oil. ${ }^{1} \mathrm{H} \mathrm{NMR}\left(\mathrm{CDCl}_{3}, 400 \mathrm{MHz}\right): \delta 8.73-$ $8.72(\mathrm{~m}, 2 \mathrm{H}), 8.07-8.05(\mathrm{~d}, J=9.0 \mathrm{~Hz}, 1 \mathrm{H}), 7.45-7.41(\mathrm{~m}, 2 \mathrm{H}), 7.38-7.27(\mathrm{~m}, 5 \mathrm{H}), 7.20-7.19$ (d, $J=4.5 \mathrm{~Hz}, 1 \mathrm{H}), 6.16-6.14$ (t, $J=3.7 \mathrm{~Hz}, 1 \mathrm{H}), 4.03$ (s, 3H), 4.01-3.99 (m, 1H), 3.94-3.89 $(\mathrm{m}, 1 \mathrm{H}), 2.56-2.54(\mathrm{~m}, 2 \mathrm{H}), 2.37-2.31\left(\mathrm{ddd}, J_{l}=2.2 \mathrm{~Hz}, J_{2}=7.7 \mathrm{~Hz}, J_{3}=17.1 \mathrm{~Hz}, 1 \mathrm{H}\right), 2.24-$ $2.14(\mathrm{~m}, 1 \mathrm{H}), 2.11-2.05\left(\mathrm{ddd}, J_{1}=1.8 \mathrm{~Hz}, J_{2}=4.1 \mathrm{~Hz}, J_{3}=17.1 \mathrm{~Hz}, 1 \mathrm{H}\right), 2.00-1.98(\mathrm{~m}, 1 \mathrm{H})$; ${ }^{13} \mathrm{C} \mathrm{NMR}\left(\mathrm{CDCl}_{3}, 100 \mathrm{MHz}\right): \delta 199.5,158.0,147.5,147.4,144.3,140.6,140.5,132.1,128.7$, 128.2, 127.6, 126.4, 126.2, 121.3, 119.2, 101.4, 55.6, 44.6, 38.8, 35.0, 26.6, 22.1; HRMS (EI) $\mathrm{m} / \mathrm{z}$ : Calcd for $\mathrm{C}_{24} \mathrm{H}_{23} \mathrm{NO}_{2} 357.1729$; found 357.1732; HPLC (Chiralpak AD-3, $i-\mathrm{PrOH} /$ hexane $=20 / 80$, flow rate $=1.0 \mathrm{~mL} / \mathrm{min}, \lambda=254 \mathrm{~nm}): \mathrm{t}_{\text {minor }}=14.1, \mathrm{t}_{\text {major }}=12.0$, ee $=99 \%$.<smiles>O=CC[C@@H]1C(c2ccccc2)=CCCC1c1ccnc2cc(Cl)ccc12</smiles>

2-((2R,3S)-3-(7-Chloroquinolin-4-yl)-2,3,4,5-tetrahydro-[1,1'-biphenyl]-2-

yl)acetaldehyde (3c): The title compound was prepared according to the general procedure, as described above in $42 \%$ yield $(30 \mathrm{mg})$ as yellow oil. ${ }^{1} \mathrm{H} \mathrm{NMR}\left(\mathrm{CDCl}_{3}, 400 \mathrm{MHz}\right): \delta 9.56-9.56$ (t, $J=1.4 \mathrm{~Hz}, 1 \mathrm{H}), 8.83-8.82(\mathrm{~d}, J=4.6 \mathrm{~Hz}, 1 \mathrm{H}), 8.14-8.14$ (d, $J=2.2 \mathrm{~Hz}, 1 \mathrm{H}), 8.03-8.01$ (d, $J=9.1 \mathrm{~Hz}, 1 \mathrm{H}), 7.55-7.52\left(\mathrm{dd}, J_{l}=2.2 \mathrm{~Hz}, J_{2}=9.1 \mathrm{~Hz}, 1 \mathrm{H}\right), 7.42-7.36(\mathrm{~m}, 5 \mathrm{H}), 7.33-7.29(\mathrm{~m}$, 
$1 \mathrm{H}), 6.13-6.11(\mathrm{t}, J=3.3 \mathrm{~Hz}, 1 \mathrm{H}), 3.79-3.76(\mathrm{~m}, 1 \mathrm{H}), 3.70-3.69(\mathrm{~m}, 1 \mathrm{H}), 2.67-2.60$ (ddd, $J_{1}=$ $\left.1.7 \mathrm{~Hz}, J_{2}=8.3 \mathrm{~Hz}, J_{3}=17.7 \mathrm{~Hz}, 1 \mathrm{H}\right), 2.51-2.46\left(\mathrm{ddd}, J_{1}=1.1 \mathrm{~Hz}, J_{2}=3.1 \mathrm{~Hz}, J_{3}=17.7 \mathrm{~Hz}\right.$, $1 \mathrm{H}), 2.30-2.23(\mathrm{~m}, 1 \mathrm{H}), 2.07-1.92(\mathrm{~m}, 3 \mathrm{H}) ;{ }^{13} \mathrm{C} \mathrm{NMR}\left(\mathrm{CDCl}_{3}, 100 \mathrm{MHz}\right): \delta 201.0,151.2,150.6$, 149.0, 141.0, 139.3, 135.0, 129.4, 128.8, 127.8, 127.6, 127.5, 126.3, 125.4, 124.4, 118.8, 47.6, 38.0, 35.6, 25.4, 23.4; HRMS (EI) $\mathrm{m} / \mathrm{z}$ : Calcd for $\mathrm{C}_{23} \mathrm{H}_{20} \mathrm{CINO} 361.1233$; found 361.1236; HPLC (Chiralpak AD-3, $i$-PrOH $/$ hexane $=20 / 80$, flow rate $=1.0 \mathrm{~mL} / \mathrm{min}, \lambda=254 \mathrm{~nm}$ ): $t_{\text {minor }}$ $=9.9, \mathrm{t}_{\text {major }}=11.3$, ee $=98 \%$.

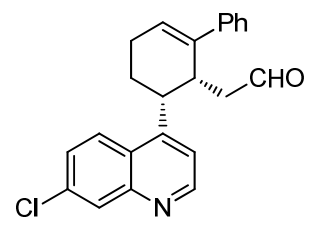

2-((2R,3R)-3-(7-Chloroquinolin-4-yl)-2,3,4,5-tetrahydro-[1,1'-biphenyl]-2-

yl)acetaldehyde ( $\left.\mathbf{3} \mathbf{c}^{\prime}\right)$ : The title compound was prepared according to the general procedure, as described above in $37 \%$ yield $(26 \mathrm{mg})$ as yellow oil. ${ }^{1} \mathrm{H} \mathrm{NMR}\left(\mathrm{CDCl}_{3}, 400 \mathrm{MHz}\right): \delta 8.86-$ $8.85(\mathrm{~d}, J=4.5 \mathrm{~Hz}, 1 \mathrm{H}), 8.76-8.75(\mathrm{t}, J=1.7 \mathrm{~Hz}, 1 \mathrm{H}), 8.21-8.18(\mathrm{~d}, J=9.1 \mathrm{~Hz}, 1 \mathrm{H}), 8.15-8.14$ $(\mathrm{d}, J=2.1 \mathrm{~Hz}, 1 \mathrm{H}), 7.66-7.63\left(\mathrm{dd}, J_{1}=2.1 \mathrm{~Hz}, J_{2}=9.1 \mathrm{~Hz}, 1 \mathrm{H}\right), 7.38-7.36(\mathrm{~m}, 2 \mathrm{H}), 7.34-7.30$ $(\mathrm{m}, 2 \mathrm{H}), 7.28-7.26(\mathrm{~m}, 1 \mathrm{H}), 7.23-7.22(\mathrm{~d}, J=4.6 \mathrm{~Hz}, 1 \mathrm{H}), 6.17-6.16(\mathrm{t}, J=3.7 \mathrm{~Hz}, 1 \mathrm{H}), 4.00-$ $3.94(\mathrm{~m}, 2 \mathrm{H}), 2.54-2.52(\mathrm{~m}, 2 \mathrm{H}), 2.40-2.34\left(\mathrm{ddd}, J_{1}=2.0 \mathrm{~Hz}, J_{2}=7.7 \mathrm{~Hz}, J_{3}=17.5 \mathrm{~Hz}, 1 \mathrm{H}\right)$, $2.22-2.13(\mathrm{~m}, 1 \mathrm{H}), 2.11-2.06\left(\mathrm{ddd}, J_{l}=1.4 \mathrm{~Hz}, J_{2}=3.5 \mathrm{~Hz}, J_{3}=17.5 \mathrm{~Hz}, 1 \mathrm{H}\right), 1.99-1.97(\mathrm{~m}$, $1 \mathrm{H}) ;{ }^{13} \mathrm{C} \mathrm{NMR}\left(\mathrm{CDCl}_{3}, 100 \mathrm{MHz}\right): \delta 199.1,151.0,149.4,148.7,140.4,140.2,135.2,129.5$, 128.7, 127.9, 127.6, 126.3, 126.2, 125.8, 124.3, 119.1, 44.5, 38.7, 35.4, 26.5, 22.1; HRMS (EI) $\mathrm{m} / \mathrm{z}$ : Calcd for $\mathrm{C}_{23} \mathrm{H}_{20} \mathrm{CINO}$ 361.1233; Found 361.1237; HPLC (Chiralpak AD-3, $i$ $\mathrm{PrOH} /$ hexane $=20 / 80$, flow rate $=1.0 \mathrm{~mL} / \mathrm{min}, \lambda=254 \mathrm{~nm}): \mathrm{t}_{\text {minor }}=7.9, \mathrm{t}_{\text {major }}=10.4$, ee $=99 \%$.<smiles>O=CC[C@H]1C(c2ccccc2)=CCCC1c1ccnc2cc(C(F)(F)F)ccc12</smiles>

2-((2R,3S)-3-(7-(Trifluoromethyl)quinolin-4-yl)-2,3,4,5-tetrahydro-[1,1'-biphenyl]-2yl)acetaldehyde (3d): The title compound was prepared according to the general procedure, as described above in $18 \%$ yield $(14 \mathrm{mg})$ as yellow oil. ${ }^{1} \mathrm{H} \mathrm{NMR}\left(\mathrm{CDCl}_{3}, 400 \mathrm{MHz}\right): \delta 9.59$ (s, $1 \mathrm{H}), 8.93-8.92(\mathrm{~d}, J=4.5 \mathrm{~Hz}, 1 \mathrm{H}), 8.45(\mathrm{~s}, 1 \mathrm{H}), 8.22-8.20(\mathrm{~d}, J=8.9 \mathrm{~Hz}, 1 \mathrm{H}), 7.77-7.74(\mathrm{dd}$, $\left.J_{1}=1.4 \mathrm{~Hz}, J_{2}=8.9 \mathrm{~Hz}, 1 \mathrm{H}\right), 7.54-7.52(\mathrm{~d}, J=4.6 \mathrm{~Hz}, 1 \mathrm{H}), 7.43-7.37(\mathrm{~m}, 4 \mathrm{H}), 7.34-7.30(\mathrm{t}, J$ $=6.8 \mathrm{~Hz}, 1 \mathrm{H}), 6.14(\mathrm{~s}, 1 \mathrm{H}), 3.86-3.81(\mathrm{~m}, 1 \mathrm{H}), 3.74-3.71(\mathrm{~m}, 1 \mathrm{H}), 2.71-2.64\left(\mathrm{ddd}, J_{1}=1.6 \mathrm{~Hz}\right.$, $\left.J_{2}=8.5 \mathrm{~Hz}, J_{3}=18.1 \mathrm{~Hz}, 1 \mathrm{H}\right), 2.55-2.50\left(\mathrm{dd}, J_{1}=2.7 \mathrm{~Hz}, J_{2}=17.7 \mathrm{~Hz}, 1 \mathrm{H}\right), 2.32-2.25(\mathrm{~m}$, $1 \mathrm{H}), 2.12-2.05(\mathrm{~m}, 1 \mathrm{H}), 2.00-1.94(\mathrm{~m}, 2 \mathrm{H}) ;{ }^{13} \mathrm{C} \mathrm{NMR}\left(\mathrm{CDCl}_{3}, 100 \mathrm{MHz}\right): \delta 201.0,151.6,150.5$, $147.6,140.8,139.2,128.8,128.5,128.4,128.4,128.3,127.8,127.6,126.2,124.4,122.2,122.2$, 120.3 , 47.7, 37.9, 35.3, 25.1, 23.2; HRMS (EI) m/z: Calcd for $\mathrm{C}_{24} \mathrm{H}_{20} \mathrm{~F}_{3} \mathrm{NO} 395.1497$; Found 395.1504; HPLC (Chiralpak AD-3, $i$-PrOH $/$ hexane $=20 / 80$, flow rate $=1.0 \mathrm{~mL} / \mathrm{min}, \lambda=254$ $\mathrm{nm}): \mathrm{t}_{\text {minor }}=9.9, \mathrm{t}_{\text {major }}=11.3$, ee $=98 \%$. 
<smiles>O=CC[C@H]1C(c2ccccc2)=CCC[C@H]1c1ccnc2cc(C(F)(F)F)ccc12</smiles>

2-((2R,3R)-3-(7-(Trifluoromethyl)quinolin-4-yl)-2,3,4,5-tetrahydro-[1,1'-biphenyl]-2yl)acetaldehyde (3d'): The title compound was prepared according to the general procedure, as described above in $14 \%$ yield $(11 \mathrm{mg})$ as yellow oil. ${ }^{1} \mathrm{H} \mathrm{NMR}\left(\mathrm{CDCl}_{3}, 400 \mathrm{MHz}\right)$ : $\delta$ 8.97$8.95(\mathrm{~d}, J=4.5 \mathrm{~Hz}, 1 \mathrm{H}), 8.77(\mathrm{~s}, 1 \mathrm{H}), 8.46(\mathrm{~s}, 1 \mathrm{H}), 8.40-8.38(\mathrm{~d}, J=8.8 \mathrm{~Hz}, 1 \mathrm{H}), 7.88-7.86$ $\left(\mathrm{dd}, J_{l}=1.6 \mathrm{~Hz}, J_{2}=8.8 \mathrm{~Hz}, 1 \mathrm{H}\right), 7.39-7.37(\mathrm{~d}, J=8.6 \mathrm{~Hz}, 2 \mathrm{H}), 7.35-7.31(\mathrm{~m}, 3 \mathrm{H}), 6.19-6.17$ $(\mathrm{t}, J=3.7 \mathrm{~Hz}, 1 \mathrm{H}), 4.05-4.01(\mathrm{~m}, 1 \mathrm{H}), 3.99-3.96(\mathrm{~m}, 1 \mathrm{H}), 2.56-2.55(\mathrm{~m}, 2 \mathrm{H}), 2.42-2.36$ (ddd, $\left.J_{l}=1.9 \mathrm{~Hz}, J_{2}=7.9 \mathrm{~Hz}, J_{3}=17.5 \mathrm{~Hz}, 1 \mathrm{H}\right), 2.25-2.16(\mathrm{~m}, 1 \mathrm{H}), 2.14-2.09$ (ddd, $J_{1}=1.1 \mathrm{~Hz}, J_{2}$ $\left.=3.5 \mathrm{~Hz}, J_{3}=17.5 \mathrm{~Hz}, 1 \mathrm{H}\right), 2.01-1.99(\mathrm{~m}, 1 \mathrm{H}) ;{ }^{13} \mathrm{C} \mathrm{NMR}\left(\mathrm{CDCl}_{3}, 100 \mathrm{MHz}\right): \delta 199.1,151.3$, 149.5, 147.4, 140.4, 140.2 , 128.7, 128.5, 128.4, 127.6, 126.3, 126.3, 124.3, 122.6, 122.5, 120.6, 44.5, 38.7, 35.3, 26.5, 22.1; HRMS (EI) m/z: Calcd for $\mathrm{C}_{24} \mathrm{H}_{20} \mathrm{~F}_{3} \mathrm{NO} 395.1497$; Found 395.1495; HPLC (Chiralpak AD-3, $i$-PrOH/hexane $=20 / 80$, flow rate $=1.0 \mathrm{~mL} / \mathrm{min}, \lambda=254 \mathrm{~nm}$ ): $\mathrm{t}_{\text {minor }}$ $=7.9, \mathrm{t}_{\text {major }}=10.4$, ee $=99 \%$.<smiles>O=CCC1C(c2ccccc2)=CCCC1c1ccnc2ccc(F)cc12</smiles>

\section{2-((2R,3S)-3-(6-Fluoroquinolin-4-yl)-2,3,4,5-tetrahydro-[1,1'-biphenyl]-2-}

yl)acetaldehyde (3e): The title compound was prepared according to the general procedure, as described above in $34 \%$ yield $(23 \mathrm{mg})$ as yellow oil. ${ }^{1} \mathrm{H} \mathrm{NMR}\left(\mathrm{CDCl}_{3}, 400 \mathrm{MHz}\right): \delta 9.58(\mathrm{~s}$, $1 \mathrm{H}), 8.80-8.79(\mathrm{~d}, J=4.5 \mathrm{~Hz}, 1 \mathrm{H}), 8.16-8.12\left(\mathrm{dd}, J_{l}=5.7 \mathrm{~Hz}, J_{2}=9.2 \mathrm{~Hz}, 1 \mathrm{H}\right), 7.69-7.66$ (dd, $\left.J_{l}=2.6 \mathrm{~Hz}, J_{2}=11.1 \mathrm{~Hz}, 1 \mathrm{H}\right), 7.51-7.46\left(\mathrm{dt}, J_{1}=2.6 \mathrm{~Hz}, J_{2}=9.2 \mathrm{~Hz}, 1 \mathrm{H}\right), 7.44-7.36(\mathrm{~m}, 5 \mathrm{H})$, 7.33-7.29 (t, $J=7.1 \mathrm{~Hz}, 1 \mathrm{H}), 6.12(\mathrm{~s}, 1 \mathrm{H}), 3.72-3.72(\mathrm{~m}, 1 \mathrm{H}), 3.67-3.65(\mathrm{~m}, 1 \mathrm{H}), 2.68-2.61$ $\left(\mathrm{dd}, J_{1}=8.3 \mathrm{~Hz}, J_{2}=17.9 \mathrm{~Hz}, 1 \mathrm{H}\right), 2.54-2.49\left(\mathrm{dd}, J_{1}=3.0 \mathrm{~Hz}, J_{2}=17.9 \mathrm{~Hz}, 1 \mathrm{H}\right), 2.30-2.22$ (m, 1H), 2.08-1.93 (m, 3H); ${ }^{13} \mathrm{C} \mathrm{NMR}\left(\mathrm{CDCl}_{3}, 100 \mathrm{MHz}\right): \delta 200.9,161.9,159.4,149.7,149.7$, 149.5, 149.5, 145.6, 141.0, 139.3, 133.1, 133.0, 128.8, 127.8, 127.7, 127.6, 127.5, 126.3, 119.3, 119.2, 119.0, 106.8, 106.6, 47.8, 38.2, 35.3, 24.9, 23.3; HRMS (EI) m/z: Calcd for $\mathrm{C}_{23} \mathrm{H}_{20} \mathrm{FNO}$ 345.1529; Found 345.1533; HPLC (Chiralpak AD-3, $i-\mathrm{PrOH} /$ hexane $=20 / 80$, flow rate $=1.0$ $\mathrm{mL} / \mathrm{min}, \lambda=254 \mathrm{~nm}): \mathrm{t}_{\text {minor }}=8.4, \mathrm{t}_{\text {major }}=11.8$, ee $=99 \%$.<smiles>O=CCC1C(c2ccccc2)=CCCC1c1ccnc2ccc(F)cc12</smiles>

\section{2-((2R,3R)-3-(6-Fluoroquinolin-4-yl)-2,3,4,5-tetrahydro-[1,1'-biphenyl]-2-}

yl)acetaldehyde (3e'): The title compound was prepared according to the general procedure, as described above in $44 \%$ yield $(30 \mathrm{mg})$ as yellow oil. ${ }^{1} \mathrm{H} \mathrm{NMR}\left(\mathrm{CDCl}_{3}, 400 \mathrm{MHz}\right): \delta 8.83-$ 
$8.82(\mathrm{~d}, J=4.5 \mathrm{~Hz}, 1 \mathrm{H}), 8.77-8.76(\mathrm{t}, J=1.7 \mathrm{~Hz}, 1 \mathrm{H}), 8.17-8.14\left(\mathrm{dd}, J_{l}=5.7 \mathrm{~Hz}, J_{2}=9.2 \mathrm{~Hz}\right.$, $1 \mathrm{H}), 7.85-7.82\left(\mathrm{dd}, J_{1}=2.7 \mathrm{~Hz}, J_{2}=10.3 \mathrm{~Hz}, 1 \mathrm{H}\right), 7.56-7.51\left(\mathrm{ddd}, J_{1}=2.7 \mathrm{~Hz}, J_{2}=8.0 \mathrm{~Hz}, J_{3}\right.$ $=9.2 \mathrm{~Hz}, 1 \mathrm{H}), 7.39-7.37(\mathrm{~m}, 2 \mathrm{H}), 7.35-7.31(\mathrm{~m}, 2 \mathrm{H}), 7.28-7.24(\mathrm{~m}, 2 \mathrm{H}), 6.17-6.15(\mathrm{t}, J=3.8$ $\mathrm{Hz}, 1 \mathrm{H}), 3.93-3.92(\mathrm{~m}, 1 \mathrm{H}), 3.90-3.86(\mathrm{~m}, 1 \mathrm{H}), 2.55-2.52(\mathrm{~m}, 2 \mathrm{H}), 2.40-2.33$ (ddd, $J_{l}=2.0 \mathrm{~Hz}$, $\left.J_{2}=7.7 \mathrm{~Hz}, J_{3}=17.3 \mathrm{~Hz}, 1 \mathrm{H}\right), 2.23-2.14(\mathrm{~m}, 1 \mathrm{H}), 2.12-2.07\left(\mathrm{ddd}, J_{1}=1.5 \mathrm{~Hz}, J_{2}=3.7 \mathrm{~Hz}, J_{3}\right.$ $=17.3 \mathrm{~Hz}, 1 \mathrm{H}), 1.99-1.96(\mathrm{~m}, 1 \mathrm{H}) ;{ }^{13} \mathrm{C} \mathrm{NMR}\left(\mathrm{CDCl}_{3}, 100 \mathrm{MHz}\right): \delta 199.3,162.1,159.6,149.2$, $149.2,148.7,148.6,145.4,140.5,140.3,133.2,133.1,128.7,128.2,128.2 .127 .6,126.4,126.2$, 119.6, 119.5, 119.3, 106.8, 106.5, 44.6, 38.8, 35.2, 26.5, 22.1; HRMS (EI) m/z: Calcd for $\mathrm{C}_{23} \mathrm{H}_{20} \mathrm{FNO} 345.1529$; Found 345.1532; HPLC (Chiralpak AD-3, $i$-PrOH/hexane $=10 / 90$, flow rate $=1.0 \mathrm{~mL} / \mathrm{min}, \lambda=254 \mathrm{~nm}): \mathrm{t}_{\text {minor }}=8.1, \mathrm{t}_{\text {major }}=9.7$, ee $=98 \%$.<smiles>Cc1ccc2nccc(C3CCC=C(c4ccccc4)C3C=O)c2c1</smiles>

2-((2R,3S)-3-(6-Methylquinolin-4-yl)-2,3,4,5-tetrahydro-[1,1'-biphenyl]-2-

yl)acetaldehyde (3f): The title compound was prepared according to the general procedure, as described above in $42 \%$ yield $(29 \mathrm{mg})$ as yellow oil. ${ }^{1} \mathrm{H} \mathrm{NMR}\left(\mathrm{CDCl}_{3}, 400 \mathrm{MHz}\right): \delta 9.58-9.57$ (t, $J=1.4 \mathrm{~Hz}, 1 \mathrm{H}), 8.77-8.75(\mathrm{~d}, J=4.6 \mathrm{~Hz}, 1 \mathrm{H}), 8.04-8.02(\mathrm{~d}, J=8.6 \mathrm{~Hz}, 1 \mathrm{H}), 7.81(\mathrm{~s}, 1 \mathrm{H})$, 7.56-7.53 (dd, $\left.J_{1}=1.7 \mathrm{~Hz}, J_{2}=8.6 \mathrm{~Hz}, 1 \mathrm{H}\right), 7.43-7.36(\mathrm{~m}, 5 \mathrm{H}), 7.33-7.30(\mathrm{~m}, 1 \mathrm{H}), 6.13-6.12$ $(\mathrm{t}, J=3.4 \mathrm{~Hz}, 1 \mathrm{H}), 3.81-3.77(\mathrm{~m}, 1 \mathrm{H}), 3.73-3.69(\mathrm{~m}, 1 \mathrm{H}), 2.66-2.59\left(\mathrm{ddd}, J_{l}=1.9 \mathrm{~Hz}, J_{2}=8.3\right.$ $\left.\mathrm{Hz}, J_{3}=17.6 \mathrm{~Hz}, 1 \mathrm{H}\right), 2.58(\mathrm{~s}, 3 \mathrm{H}), 2.52-2.47\left(\mathrm{ddd}, J_{1}=1.4 \mathrm{~Hz}, J_{2}=3.3 \mathrm{~Hz}, J_{3}=17.7 \mathrm{~Hz}, 1 \mathrm{H}\right)$,

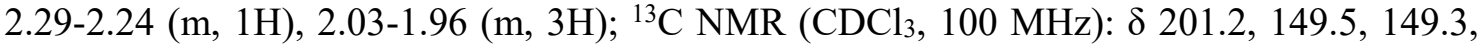
146.9, 141.1, 139.4, 136.4, 131.2, 130.2, 128.7, 127.9, 127.4, 126.8, 126.3, 121.8, 118.5, 47.7, 37.8, 35.7, 25.3, 23.4, 22.1; HRMS (EI) m/z: Calcd for $\mathrm{C}_{24} \mathrm{H}_{23} \mathrm{NO} 341.1780$; Found 341.1782; HPLC (Chiralpak AD-3, $i$-PrOH/hexane $=20 / 80$, flow rate $=1.0 \mathrm{~mL} / \mathrm{min}, \lambda=254 \mathrm{~nm}$ ): $t_{\text {minor }}$ $=9.6, \mathrm{t}_{\text {major }}=11.6$, ee $=98 \%$.<smiles>Cc1ccc2nccc(C3CCC=C(c4ccccc4)C3C=O)c2c1</smiles>

2-((2R,3R)-3-(6-Methylquinolin-4-yl)-2,3,4,5-tetrahydro-[1,1'-biphenyl]-2-

yl)acetaldehyde (3f'): The title compound was prepared according to the general procedure, as described above in $45 \%$ yield $(31 \mathrm{mg})$ as yellow oil. ${ }^{1} \mathrm{H} \mathrm{NMR}\left(\mathrm{CDCl}_{3}, 400 \mathrm{MHz}\right): \delta 8.80-$ $8.79(\mathrm{~d}, J=4.6 \mathrm{~Hz}, 1 \mathrm{H}), 8.74-8.73(\mathrm{t}, J=1.9 \mathrm{~Hz}, 1 \mathrm{H}), 8.08-8.06$ (d, $J=8.6 \mathrm{~Hz}, 1 \mathrm{H}), 7.98$ (s, $1 \mathrm{H}), 7.61-7.59\left(\mathrm{dd}, J_{l}=1.7 \mathrm{~Hz}, J_{2}=8.6 \mathrm{~Hz}, 1 \mathrm{H}\right), 7.39-7.27(\mathrm{~m}, 5 \mathrm{H}), 7.22-7.21(\mathrm{~d}, J=4.6 \mathrm{~Hz}$, $1 \mathrm{H}), 6.18-6.16(\mathrm{t}, J=3.6 \mathrm{~Hz}, 1 \mathrm{H}), 4.03-3.98(\mathrm{~m}, 1 \mathrm{H}), 3.97-3.93(\mathrm{~m}, 1 \mathrm{H}), 2.66(\mathrm{~s}, 3 \mathrm{H}), 2.56-$ $2.53(\mathrm{~m}, 1 \mathrm{H}), 2.39-2.33\left(\mathrm{ddd}, J_{1}=2.1 \mathrm{~Hz}, J_{2}=7.7 \mathrm{~Hz}, J_{3}=17.1 \mathrm{~Hz}, 1 \mathrm{H}\right), 2.20-2.10(\mathrm{~m}, 1 \mathrm{H})$,

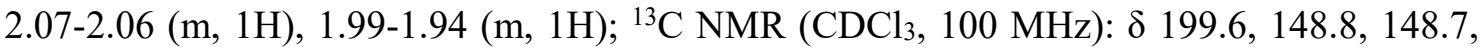
146.5, 140.6, 140.4, 136.9, 131.6, 130.1, 128.7, 127.6, 127.2, 126.4, 126.3, 121.7, 118.9, 44.6, 38.5, 35.6, 26.6, 22.3, 22.1; HRMS (EI) m/z: Calcd for $\mathrm{C}_{24} \mathrm{H}_{23} \mathrm{NO} 341.1780$; Found 341.1783; 
HPLC (Chiralpak AD-3, $i$-PrOH/hexane $=20 / 80$, flow rate $=1.0 \mathrm{~mL} / \mathrm{min}, \lambda=254 \mathrm{~nm}): \mathrm{t}_{\text {minor }}$ $=10.1, \mathrm{t}_{\text {major }}=8.3$, ee $=98 \%$.<smiles>Cc1cc(C2CCC=C(c3ccccc3)C2C=O)c2ccccc2n1</smiles>

2-((2R,3S)-3-(2-Methylquinolin-4-yl)-2,3,4,5-tetrahydro-[1,1'-biphenyl]-2-

yl)acetaldehyde (3g): The title compound was prepared according to the general procedure, as described above in $33 \%$ yield $(23 \mathrm{mg})$ as yellow oil. ${ }^{1} \mathrm{H} \mathrm{NMR}\left(\mathrm{CDCl}_{3}, 400 \mathrm{MHz}\right)$ : $\delta$ 9.53$9.52(\mathrm{t}, J=1.4 \mathrm{~Hz}, 1 \mathrm{H}), 8.07-8.04(\mathrm{~d}, J=8.4 \mathrm{~Hz}, 1 \mathrm{H}), 8.03-8.01(\mathrm{~d}, J=9.3 \mathrm{~Hz}, 1 \mathrm{H}), 7.70-7.66$ $\left(\mathrm{ddd}, J_{1}=1.2 \mathrm{~Hz}, J_{2}=7.0 \mathrm{~Hz}, J_{3}=8.2 \mathrm{~Hz}, 1 \mathrm{H}\right), 7.53-7.49\left(\mathrm{ddd}, J_{1}=1.2 \mathrm{~Hz}, J_{2}=7.0 \mathrm{~Hz}, J_{3}=\right.$ $8.2 \mathrm{~Hz}, 1 \mathrm{H}), 7.42-7.36(\mathrm{~m}, 4 \mathrm{H}), 7.32-7.28(\mathrm{~m}, 2 \mathrm{H}), 6.11(\mathrm{t}, J=2.2 \mathrm{~Hz}, 1 \mathrm{H}), 3.78-3.76(\mathrm{~m}, 1 \mathrm{H})$, 3.70 (brs, $1 \mathrm{H}), 2.70(\mathrm{~s}, 3 \mathrm{H}), 2.63-2.56\left(\mathrm{ddd}, J_{I}=1.7 \mathrm{~Hz}, J_{2}=7.9 \mathrm{~Hz}, J_{3}=17.7 \mathrm{~Hz}, 1 \mathrm{H}\right), 2.48-$ $2.42\left(\mathrm{ddd}, J_{1}=1.3 \mathrm{~Hz}, J_{2}=3.3 \mathrm{~Hz}, J_{3}=17.7 \mathrm{~Hz}, 1 \mathrm{H}\right), 2.31-2.25(\mathrm{~m}, 1 \mathrm{H}), 2.05-1.96(\mathrm{~m}, 3 \mathrm{H})$;

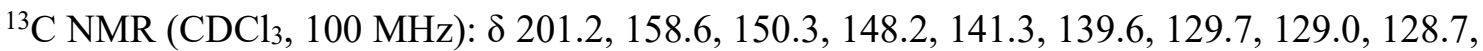
127.9, 127.4, 126.4, 125.7, 125.2, 122.7, 119.4, 47.4, 38.1, 36.0, 25.9, 25.6, 23.8; HRMS (EI) $\mathrm{m} / \mathrm{z}$ : Calcd for $\mathrm{C}_{24} \mathrm{H}_{23} \mathrm{NO} 341.1780$; Found 341.1779; HPLC (Chiralpak OD-3, $i$-PrOH/hexane $=15 / 85$, flow rate $=1.0 \mathrm{~mL} / \mathrm{min}, \lambda=254 \mathrm{~nm}): t_{\text {minor }}=13.1, \mathrm{t}_{\text {major }}=19.3$, ee $=98 \%$.<smiles>Cc1cc(C2CCC=C(c3ccccc3)C2C=O)c2ccccc2n1</smiles>

\section{2-((2R,3R)-3-(2-Methylquinolin-4-yl)-2,3,4,5-tetrahydro-[1,1'-biphenyl]-2-}

yl)acetaldehyde (3g'): The title compound was prepared according to the general procedure, as described above in $56 \%$ yield $(38 \mathrm{mg})$ as yellow oil. ${ }^{1} \mathrm{H} \mathrm{NMR}\left(\mathrm{CDCl}_{3}, 400 \mathrm{MHz}\right): \delta 8.70-$ $8.69(\mathrm{t}, J=2.0 \mathrm{~Hz}, 1 \mathrm{H}), 8.20-8.18(\mathrm{~d}, J=8.2 \mathrm{~Hz}, 1 \mathrm{H}), 8.08-8.06(\mathrm{~d}, J=8.4 \mathrm{~Hz}, 1 \mathrm{H}), 7.74-7.70$ $\left(\mathrm{dt}, J_{l}=1.2 \mathrm{~Hz}, J_{2}=6.9 \mathrm{~Hz}, 1 \mathrm{H}\right), 7.64-7.60\left(\mathrm{dt}, J_{l}=1.1 \mathrm{~Hz}, J_{2}=7.0 \mathrm{~Hz}, 1 \mathrm{H}\right), 7.39-7.36(\mathrm{~m}$, 2H), 7.34-7.30 (m, 2H), 7.27-7.23 (m, 1H), $7.11(\mathrm{~s}, 1 \mathrm{H}), 6.15-6.14(\mathrm{t}, J=3.8 \mathrm{~Hz}, 1 \mathrm{H}), 4.02-$ $3.97(\mathrm{~m}, 1 \mathrm{H}), 3.93-3.92(\mathrm{~m}, 1 \mathrm{H}), 2.74(\mathrm{~s}, 3 \mathrm{H}), 2.54-2.53(\mathrm{~m}, 2 \mathrm{H}), 2.36-2.30$ (ddd, $J_{1}=2.3 \mathrm{~Hz}$, $\left.J_{2}=7.7 \mathrm{~Hz}, J_{3}=17.0 \mathrm{~Hz}, 1 \mathrm{H}\right), 2.21-2.12(\mathrm{~m}, 1 \mathrm{H}), 2.10-2.05\left(\mathrm{ddd}, J_{1}=1.9 \mathrm{~Hz}, J_{2}=4.2 \mathrm{~Hz}, J_{3}\right.$ $=17.0 \mathrm{~Hz}, 1 \mathrm{H}), 1.98-1.95(\mathrm{~m}, 1 \mathrm{H}) ;{ }^{13} \mathrm{C} \mathrm{NMR}\left(\mathrm{CDCl}_{3}, 100 \mathrm{MHz}\right): \delta 199.8,158.5,148.9,148.0$, $140.6,140.5,129.8,129.2,128.6,127.5,126.3,126.3,126.0,125.4,122.6,119.9,44.6,38.5$, 35.8, 26.6, 25.5, 22.0; HRMS (EI) m/z: Calcd for $\mathrm{C}_{24} \mathrm{H}_{23} \mathrm{NO} 341.1780$; Found 341.1781; HPLC $($ Chiralpak OD-3, $i-\mathrm{PrOH} /$ hexane $=15 / 85$, flow rate $=1.0 \mathrm{~mL} / \mathrm{min}, \lambda=254 \mathrm{~nm}): \mathrm{t}_{\text {minor }}=11.2$, $\mathrm{t}_{\text {major }}=16.6$, ee $=89 \%$.<smiles>CC1=CCCC(c2ccnc3ccccc23)[C@@H]1CC=O</smiles> 
2-((1R,6S)-2-Methyl-6-(quinolin-4-yl)cyclohex-2-en-1-yl)acetaldehyde $(3 \mathrm{~h}):$ The title compound was prepared according to the general procedure, as described above in $47 \%$ yield $(25 \mathrm{mg})$ as yellow oil. ${ }^{1} \mathrm{H}$ NMR $\left(\mathrm{CDCl}_{3}, 400 \mathrm{MHz}\right): \delta 9.75-9.74(\mathrm{t}, J=1.6 \mathrm{~Hz}, 1 \mathrm{H}), 8.85-8.84$ $(\mathrm{d}, J=4.6 \mathrm{~Hz}, 1 \mathrm{H}), 8.15-8.13(\mathrm{~d}, J=8.3 \mathrm{~Hz}, 1 \mathrm{H}), 8.06-8.04(\mathrm{~d}, J=8.5 \mathrm{~Hz}, 1 \mathrm{H}), 7.74-7.70(\mathrm{dt}$, $\left.J_{l}=1.1 \mathrm{~Hz}, J_{2}=7.0 \mathrm{~Hz}, 1 \mathrm{H}\right), 7.59-7.55\left(\mathrm{dt}, J_{l}=1.1 \mathrm{~Hz}, J_{2}=7.1 \mathrm{~Hz}, 1 \mathrm{H}\right), 7.30-7.28(\mathrm{~d}, J=4.6$ $\mathrm{Hz}, 1 \mathrm{H}), 5.66(\mathrm{~s}, 1 \mathrm{H}), 3.68-3.63(\mathrm{~m}, 1 \mathrm{H}), 3.02-3.01(\mathrm{~m}, 1 \mathrm{H}), 2.62-2.51(\mathrm{~m}, 2 \mathrm{H}), 2.17-2.12(\mathrm{~m}$, 1H), 1.98-1.83 (m, 3H), $1.81(\mathrm{~s}, 3 \mathrm{H}) ;{ }^{13} \mathrm{C} \mathrm{NMR}\left(\mathrm{CDCl}_{3}, 100 \mathrm{MHz}\right): \delta 201.7,150.8,150.2$, $148.4,133.9,130.5,129.0,127.1,126.6,124.5,122.8,118.5,46.4,38.9,38.6,27.5,23.9,22.2$; HRMS (EI) m/z: Calcd for $\mathrm{C}_{18} \mathrm{H}_{19} \mathrm{NO}$ 265.1467; Found 265.1468; HPLC (Chiralpak AD-3, $i$ $\mathrm{PrOH} /$ hexane $=20 / 80$, flow rate $=1.0 \mathrm{~mL} / \mathrm{min}, \lambda=254 \mathrm{~nm}): \mathrm{t}_{\text {minor }}=8.8, \mathrm{t}_{\text {major }}=10.6$, ee $=97 \%$.<smiles>CC1=CCCC(c2ccnc3ccccc23)C1CC=O</smiles>

2-((1R,6R)-2-Methyl-6-(quinolin-4-yl)cyclohex-2-en-1-yl)acetaldehyde (3h'): The title compound was prepared according to the general procedure, as described above in $44 \%$ yield $(23 \mathrm{mg})$ as yellow oil. ${ }^{1} \mathrm{H} \mathrm{NMR}\left(\mathrm{CDCl}_{3}, 400 \mathrm{MHz}\right): \delta 8.95-8.94(\mathrm{~d}, J=1.1 \mathrm{~Hz}, 1 \mathrm{H}), 8.86-8.85$ $(\mathrm{d}, J=4.4 \mathrm{~Hz}, 1 \mathrm{H}), 8.14-8.12(\mathrm{~d}, J=8.6 \mathrm{~Hz}, 2 \mathrm{H}), 7.75-7.71(\mathrm{t}, J=7.1 \mathrm{~Hz}, 1 \mathrm{H}), 7.64-7.60(\mathrm{t}, J$ $=7.1 \mathrm{~Hz}, 1 \mathrm{H}), 7.22-7.21(\mathrm{~d}, J=4.4 \mathrm{~Hz}, 1 \mathrm{H}), 5.60(\mathrm{~s}, 1 \mathrm{H}), 3.89-3.86(\mathrm{~m}, 1 \mathrm{H}), 3.14-3.13(\mathrm{~m}$, $1 \mathrm{H}), 2.37-2.19(\mathrm{~m}, 3 \mathrm{H}), 2.11-2.00(\mathrm{~m}, 1 \mathrm{H}), 1.90-1.84(\mathrm{~m}, 2 \mathrm{H}), 1.76(\mathrm{~s}, 3 \mathrm{H}) ;{ }^{13} \mathrm{C} \mathrm{NMR}\left(\mathrm{CDCl}_{3}\right.$, $100 \mathrm{MHz}): \delta 200.6,150.0,149.2,148.2,135.7,130.5,129.2,127.2,126.7,123.1,122.9,119.1$, 44.2, 38.5, 38.2, 25.7, 22.5, 22.4; HRMS (EI) m/z: Calcd for $\mathrm{C}_{18} \mathrm{H}_{19} \mathrm{NO}$ 265.1467; Found 265.1469; HPLC (Chiralpak AD-3, $i$-PrOH $/$ hexane $=20 / 80$, flow rate $=1.0 \mathrm{~mL} / \mathrm{min}, \lambda=254$ $\mathrm{nm}): \mathrm{t}_{\text {minor }}=8.9, \mathrm{t}_{\text {major }}=11.3$, ee $=92 \%$.<smiles>CC1C=C(c2ccccc2)[C@H](CC=O)C(c2ccnc3ccccc23)C1</smiles>

2-((2R,3S)-5-Methyl-3-(quinolin-4-yl)-2,3,4,5-tetrahydro-[1,1'-biphenyl]-2-

yl)acetaldehyde (3i): The title compound was prepared according to the general procedure, as described above in $42 \%$ yield $(29 \mathrm{mg})$ as yellow oil. ${ }^{1} \mathrm{H} \mathrm{NMR}\left(\mathrm{CDCl}_{3}, 400 \mathrm{MHz}\right): \delta 9.66(\mathrm{~s}$, $1 \mathrm{H}), 8.81-8.80(\mathrm{~d}, J=4.6 \mathrm{~Hz}, 1 \mathrm{H}), 8.16-8.14(\mathrm{~d}, J=8.1 \mathrm{~Hz}, 1 \mathrm{H}), 8.08-8.06(\mathrm{~d}, J=8.3 \mathrm{~Hz}, 1 \mathrm{H})$, 7.74-7.70 (dt, $\left.J_{l}=1.1 \mathrm{~Hz}, J_{2}=7.1 \mathrm{~Hz}, \mathrm{H}\right), 7.61-7.57\left(\mathrm{dt}, J_{1}=1.1 \mathrm{~Hz}, J_{2}=7.1 \mathrm{~Hz}, 1 \mathrm{H}\right), 7.49-$ $7.47(\mathrm{~d}, J=7.2 \mathrm{~Hz}, 2 \mathrm{H}), 7.43-7.38(\mathrm{~m}, 3 \mathrm{H}), 7.36-7.31(\mathrm{~m}, 1 \mathrm{H}), 5.99(\mathrm{~s}, 1 \mathrm{H}), 3.86-3.85(\mathrm{~m} .1 \mathrm{H})$, 3.74-3.72 (m, $1 \mathrm{H}), 2.72-2.65\left(\mathrm{ddd}, J_{1}=1.9 \mathrm{~Hz}, J_{2}=9.1 \mathrm{~Hz}, J_{3}=17.8 \mathrm{~Hz}, 1 \mathrm{H}\right), 2.61-2.56(\mathrm{dd}$, $\left.J_{l}=2.5 \mathrm{~Hz}, J_{2}=17.8 \mathrm{~Hz}, 1 \mathrm{H}\right), 2.08-2.03(\mathrm{~m}, 1 \mathrm{H}), 2.00-1.97(\mathrm{~m}, 1 \mathrm{H}), 1.72-1.67(\mathrm{~m}, 1 \mathrm{H}), 1.08-$ $1.06(\mathrm{~d}, \mathrm{~J}=7.0 \mathrm{~Hz}, 3 \mathrm{H}) ;{ }^{13} \mathrm{C} \mathrm{NMR}\left(\mathrm{CDCl}_{3}, 100 \mathrm{MHz}\right): \delta 201.1,150.3,150.2,148.4,140.7$, 138.5, 133.9, 130.6, 128.9, 128.8, 127.6, 126.7, 126.6, 126.3, 123.0, 118.5, 48.6, 37.0, 34.6, 32.4, 27.5, 20.7; HRMS (EI) m/z: Calcd for $\mathrm{C}_{24} \mathrm{H}_{23} \mathrm{NO} 341.1780$; Found 341.1779; HPLC 
$($ Chiralpak AD-3, $i-\mathrm{PrOH} /$ hexane $=20 / 80$, flow rate $=1.0 \mathrm{~mL} / \mathrm{min}, \lambda=254 \mathrm{~nm}): \mathrm{t}_{\text {minor }}=8.1$, $\mathrm{t}_{\text {major }}=7.1$, ee $=92 \%$.<smiles>CC1C=C(c2ccccc2)[C@@H](CC=O)C(c2ccnc3ccccc23)C1</smiles>

2-((2R,3R)-5-Methyl-3-(quinolin-4-yl)-2,3,4,5-tetrahydro-[1,1'-biphenyl]-2-

yl)acetaldehyde (3i'): The title compound was prepared according to the general procedure, as described above in $49 \%$ yield $(33 \mathrm{mg})$ as yellow oil. ${ }^{1} \mathrm{H} \mathrm{NMR}\left(\mathrm{CDCl}_{3}, 400 \mathrm{MHz}\right)$ : $\delta$ 8.87$8.86(\mathrm{~d}, J=4.5 \mathrm{~Hz}, 1 \mathrm{H}), 8.70-8.69(\mathrm{t}, J=1.8 \mathrm{~Hz}, 1 \mathrm{H}), 8.27-8.25(\mathrm{~d}, J=8.1 \mathrm{~Hz}, 1 \mathrm{H}), 8.17-8.15$ $(\mathrm{d}, J=8.3 \mathrm{~Hz}, 1 \mathrm{H}), 7.79-7.75\left(\mathrm{dt}, J_{1}=1.2 \mathrm{~Hz}, J_{2}=6.9 \mathrm{~Hz}, 1 \mathrm{H}\right), 7.72-7.67\left(\mathrm{dt}, J_{1}=1.2 \mathrm{~Hz}, J_{2}\right.$ $=7.0 \mathrm{~Hz}, 1 \mathrm{H}), 7.40-7.38(\mathrm{~d}, J=7.3 \mathrm{~Hz}, 2 \mathrm{H}), 7.34-7.30(\mathrm{t}, J=7.1 \mathrm{~Hz}, 2 \mathrm{H}), 7.27-7.25(\mathrm{~m}, 1 \mathrm{H})$, 7.24-7.23 (d, $J=4.4 \mathrm{~Hz}, 1 \mathrm{H}), 5.99(\mathrm{~s}, 1 \mathrm{H}), 4.10-4.06(\mathrm{~m}, 1 \mathrm{H}), 3.95$ (brs, 1H), 2.72-2.66 (m, $1 \mathrm{H}), 2.36-2.30\left(\mathrm{ddd}, J_{1}=2.1 \mathrm{~Hz}, J_{2}=7.8 \mathrm{~Hz}, J_{3}=17.2 \mathrm{~Hz}, 1 \mathrm{H}\right), 2.07-2.00(\mathrm{~m}, 2 \mathrm{H}), 1.78-1.75$ $(\mathrm{m}, 1 \mathrm{H}), 1.27-1.26(\mathrm{~d}, J=7.1 \mathrm{~Hz}, 3 \mathrm{H}) ;{ }^{13} \mathrm{C} \mathrm{NMR}\left(\mathrm{CDCl}_{3}, 100 \mathrm{MHz}\right): \delta 199.4,150.0,149.0$, 148.2 , 140.2 139.9, 132.6, 130.6, 129.2, 128.6, 127.6, 127.3, 126.9, 126.4, 122.8, 119.0, 44.8, 38.7, 35.6, 32.6, 31.3, 21.6; HRMS (EI) m/z: Calcd for $\mathrm{C}_{24} \mathrm{H}_{23} \mathrm{NO} 341.1780$; Found 341.1781; HPLC (Chiralpak AD-3, $i$-PrOH $/$ hexane $=10 / 90$, flow rate $=1.0 \mathrm{~mL} / \mathrm{min}, \lambda=254 \mathrm{~nm}): \mathrm{t}_{\text {major }}=$ $8.2, \mathrm{t}_{\text {minor }}=10.1$, ee $=86 \%$.<smiles>CCC1=CCCC(C=O)C1c1ccnc2ccccc12</smiles>

2-((1R,6S)-2-Ethyl-6-(quinolin-4-yl)cyclohex-2-en-1-yl)acetaldehyde $\quad(3 \mathbf{j}):$ The title compound was prepared according to the general procedure, as described above in $41 \%$ yield (23 mg) as yellow oil. ${ }^{1} \mathrm{H}$ NMR $\left(\mathrm{CDCl}_{3}, 400 \mathrm{MHz}\right): \delta 9.74-9.73(\mathrm{t}, J=1.8 \mathrm{~Hz}, 1 \mathrm{H}), 8.84-8.83$ $(\mathrm{d}, J=4.6 \mathrm{~Hz}, 1 \mathrm{H}), 8.14-8.12\left(\mathrm{dd}, J_{l}=0.8 \mathrm{~Hz}, J_{2}=8.5 \mathrm{~Hz}, 1 \mathrm{H}\right), 8.05-8.03(\mathrm{~d}, J=8.4 \mathrm{~Hz}, 1 \mathrm{H})$, $7.72-7.68\left(\mathrm{ddd}, J_{l}=1.2 \mathrm{~Hz}, J_{2}=6.8 \mathrm{~Hz}, J_{3}=8.2 \mathrm{~Hz}, 1 \mathrm{H}\right), 7.58-7.54\left(\mathrm{ddd}, J_{1}=1.2 \mathrm{~Hz}, J_{2}=6.9\right.$ $\left.\mathrm{Hz}, J_{3}=8.3 \mathrm{~Hz}, 1 \mathrm{H}\right), 7.31-7.30(\mathrm{~d}, J=4.6 \mathrm{~Hz}, 1 \mathrm{H}), 5.67(\mathrm{~m}, 1 \mathrm{H}), 3.69-3.65(\mathrm{~m}, 1 \mathrm{H}), 3.06-3.04$ $(\mathrm{m}, 1 \mathrm{H}), 2.59-2.57\left(\mathrm{dd}, J_{1}=1.8 \mathrm{~Hz}, J_{2}=5.7 \mathrm{~Hz}, 2 \mathrm{H}\right), 2.19-2.05(\mathrm{~m}, 3 \mathrm{H}), 1.99-1.78(\mathrm{~m}, 3 \mathrm{H})$, $1.16-1.12(\mathrm{t}, J=7.4 \mathrm{~Hz}, 3 \mathrm{H}) ;{ }^{13} \mathrm{C} \mathrm{NMR}\left(\mathrm{CDCl}_{3}, 100 \mathrm{MHz}\right): \delta 201.8,150.8,150.2,148.4,139.3$, 130.5, 129.0, 127.1, 126.5, 122.9, 122.5, 118.5, 46.7, 38.6, 37.1, 27.8, 26.9, 23.5, 12.5; HRMS (EI) $\mathrm{m} / \mathrm{z}$ : Calcd for $\mathrm{C}_{19} \mathrm{H}_{21} \mathrm{NO}$ 279.1623; Found 279.1624; HPLC (Chiralpak AD-3, $i$ $\mathrm{PrOH} /$ hexane $=20 / 80$, flow rate $=1.0 \mathrm{~mL} / \mathrm{min}, \lambda=254 \mathrm{~nm}): \mathrm{t}_{\text {minor }}=13.0, \mathrm{t}_{\text {major }}=11.2$, ee $=93 \%$.<smiles>CCC1=CCC[C@H](c2ccnc3ccccc23)[C@@H]1CC=O</smiles> 
2-((1R,6R)-2-Ethyl-6-(quinolin-4-yl)cyclohex-2-en-1-yl)acetaldehyde $\quad\left(3 \mathbf{j} \mathbf{j}^{\prime}\right):$ The title compound was prepared according to the general procedure, as described above in $45 \%$ yield $(25 \mathrm{mg})$ as yellow oil. ${ }^{1} \mathrm{H}$ NMR $\left(\mathrm{CDCl}_{3}, 400 \mathrm{MHz}\right): \delta 8.92-8.91(\mathrm{t}, J=2.0 \mathrm{~Hz}, 1 \mathrm{H}), 8.86-8.85$ $(\mathrm{d}, J=4.5 \mathrm{~Hz}, 1 \mathrm{H}), 8.14-8.12(\mathrm{~d}, J=8.5 \mathrm{~Hz}, 1 \mathrm{H}), 8.11-8.09$ (d, $J=8.5 \mathrm{~Hz}, 1 \mathrm{H}), 7.74-7.70$ (ddd, $\left.J_{1}=1.2 \mathrm{~Hz}, J_{2}=6.9 \mathrm{~Hz}, J_{3}=8.2 \mathrm{~Hz}, 1 \mathrm{H}\right), 7.63-7.59\left(\mathrm{ddd}, J_{1}=1.2 \mathrm{~Hz}, J_{2}=7.0 \mathrm{~Hz}, J_{3}=8.3\right.$ $\mathrm{Hz}, 1 \mathrm{H}), 7.21-7.20$ (d, $J=4.5 \mathrm{~Hz}, 1 \mathrm{H}), 5.59$ (brs, $1 \mathrm{H}), 3.86-3.81(\mathrm{~m}, 1 \mathrm{H}), 3.21-3.19(\mathrm{~m}, 1 \mathrm{H})$, 2.39-2.32 (m, 3H), 2.22-2.16 (m, 1H), 2.13-2.01 (m, 2H), 1.99-1.88 (m, 2H), 1.12-1.09 (t, $J=$ $7.4 \mathrm{~Hz}, 3 \mathrm{H}) ;{ }^{13} \mathrm{C} \mathrm{NMR}\left(\mathrm{CDCl}_{3}, 100 \mathrm{MHz}\right): \delta 200.6,150.0,149.3,148.2,141.4,130.6,129.2$, 127.2, 126.7, 122.9, 120.9, 119.1, 44.6, 38.6, 36.6, 28.1, 25.7, 22.4, 12.5; HRMS (EI) m/z: Calcd for $\mathrm{C}_{19} \mathrm{H}_{21} \mathrm{NO}$ 279.1623; Found 279.1624. HPLC (Chiralpak AD-3, $i$-PrOH/hexane = 10/90, flow rate $=1.0 \mathrm{~mL} / \mathrm{min}, \lambda=254 \mathrm{~nm}): \mathrm{t}_{\text {minor }}=7.4$, $\mathrm{t}_{\text {major }}=9.2$, ee $=99 \%$.

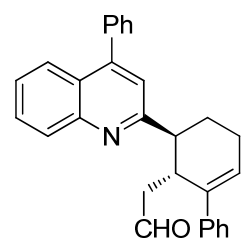

2-((2R,3S)-3-(4-Phenylquinolin-2-yl)-2,3,4,5-tetrahydro-[1,1'-biphenyl]-2-

yl)acetaldehyde (5): The title compound was prepared according to the general procedure, as described above in $31 \%$ yield $(25 \mathrm{mg})$ as yellow oil. ${ }^{1} \mathrm{H} \mathrm{NMR}\left(\mathrm{CDCl}_{3}, 400 \mathrm{MHz}\right): \delta 9.00-8.99$ $(\mathrm{q}, J=1.2 \mathrm{~Hz}, 1 \mathrm{H}), 8.14-8.12(\mathrm{~d}, J=8.3 \mathrm{~Hz}, 1 \mathrm{H}), 7.88-7.85\left(\mathrm{dd}, J_{l}=0.8 \mathrm{~Hz}, J_{2}=8.4 \mathrm{~Hz}, 1 \mathrm{H}\right)$, 7.71-7.67 (ddd, $\left.J_{l}=1.4 \mathrm{~Hz}, J_{2}=6.9 \mathrm{~Hz}, J_{3}=8.3 \mathrm{~Hz}, 1 \mathrm{H}\right), 7.55-7.49(\mathrm{~m}, 4 \mathrm{H}), 7.47-7.43(\mathrm{~m}, 4 \mathrm{H})$, 7.36-7.30 (m, 3H), 7.26-7.23 (m, 1H), 6.13-6.11 (t, $J=3.6 \mathrm{~Hz}, 1 \mathrm{H}), 4.07-4.05(\mathrm{~m}, 1 \mathrm{H}), 3.60-$ $3.55\left(\mathrm{ddd}, J_{l}=3.0 \mathrm{~Hz}, J_{2}=4.7 \mathrm{~Hz}, J_{3}=12.6 \mathrm{~Hz}, 1 \mathrm{H}\right), 2.77-2.70\left(\mathrm{ddd}, J_{l}=2.4 \mathrm{~Hz}, J_{2}=8.1 \mathrm{~Hz}\right.$, $\left.J_{3}=17.3 \mathrm{~Hz}, 1 \mathrm{H}\right), 2.49-2.46(\mathrm{~m}, 2 \mathrm{H}), 2.29-2.20(\mathrm{~m}, 1 \mathrm{H}), 2.18-2.15(\mathrm{~m}, 1 \mathrm{H}), 2.14-2.09$ (ddd, $\left.J_{1}=1.0 \mathrm{~Hz}, J_{2}=3.3 \mathrm{~Hz}, J_{3}=17.3 \mathrm{~Hz}, 1 \mathrm{H}\right) ;{ }^{13} \mathrm{C} \mathrm{NMR}\left(\mathrm{CDCl}_{3}, 100 \mathrm{MHz}\right): \delta 200.7,162.7,148.8$, 148.3, 140.8, 140.8, 138.2, 129.6, 129.4, 129.3, 128.5, 128.3, 127.2 , 126.4, 126.2, 126.1, 125.7, 125.5, 121.5, 46.0, 44.5, 35.8, 26.5, 21.5; HRMS (EI) m/z: Calcd for $\mathrm{C}_{29} \mathrm{H}_{25} \mathrm{NO} 403.1936$; Found 403.1935. HPLC (Chiralpak AD-3, $i$-PrOH $/$ hexane $=20 / 80$, flow rate $=1.0 \mathrm{~mL} / \mathrm{min}, \lambda$ $=254 \mathrm{~nm}): \mathrm{t}_{\text {minor }}=23.8, \mathrm{t}_{\text {major }}=30.7$, ee $=97 \%$.<smiles>CC1C(c2ccccc2)CCCC1c1cc(-c2ccccc2)c2ccccc2n1</smiles>

\section{2-((2R,3R)-3-(4-Phenylquinolin-2-yl)-2,3,4,5-tetrahydro-[1,1'-biphenyl]-2-}

yl)acetaldehyde (5'): The title compound was prepared according to the general procedure, as described above in $38 \%$ yield $(31 \mathrm{mg})$ as yellow oil. ${ }^{1} \mathrm{H} \mathrm{NMR}\left(\mathrm{CDCl}_{3}, 400 \mathrm{MHz}\right): \delta 9.46-9.45$ $(\mathrm{t}, J=2.1 \mathrm{~Hz}, 1 \mathrm{H}), 8.14-8.12(\mathrm{~d}, J=7.9 \mathrm{~Hz}, 1 \mathrm{H}), 7.90-7.88\left(\mathrm{dd}, J_{1}=0.9 \mathrm{~Hz}, J_{2}=8.4 \mathrm{~Hz}, 1 \mathrm{H}\right)$, 7.72-7.68 (ddd, $\left.J_{1}=1.3 \mathrm{~Hz}, J_{2}=6.8 \mathrm{~Hz}, J_{3}=8.3 \mathrm{~Hz}, 1 \mathrm{H}\right), 7.54-7.44(\mathrm{~m}, 6 \mathrm{H}), 7.40-7.32(\mathrm{~m}$, $5 \mathrm{H}), 7.27-7.24(\mathrm{~m}, 1 \mathrm{H}), 6.01-5.99(\mathrm{~m}, 1 \mathrm{H}), 4.10$ (brs, $1 \mathrm{H}), 3.27-3.22\left(\mathrm{ddd}, J_{1}=3.8 \mathrm{~Hz}, J_{2}=\right.$ $\left.7.8 \mathrm{~Hz}, J_{3}=8.3 \mathrm{~Hz}, 1 \mathrm{H}\right), 2.57-2.50\left(\mathrm{ddd}, J_{1}=2.1 \mathrm{~Hz}, J_{2}=7.2 \mathrm{~Hz}, J_{3}=17.0 \mathrm{~Hz}, 1 \mathrm{H}\right), 2.44-2.38$ $\left(\mathrm{ddd}, J_{1}=2.2 \mathrm{~Hz}, J_{2}=3.8 \mathrm{~Hz}, J_{3}=17.0 \mathrm{~Hz}, 1 \mathrm{H}\right), 2.37-2.24(\mathrm{~m}, 2 \mathrm{H}), 2.20-2.07(\mathrm{~m}, 2 \mathrm{H}) ;{ }^{13} \mathrm{C}$ 
$\mathrm{NMR}\left(\mathrm{CDCl}_{3}, 100 \mathrm{MHz}\right): \delta 202.1,163.4,148.9,148.3,142.1,140.3,138.2,129.6,129.5,129.2$, 128.5, 128.5, 128.3, 127.6, 127.0, 126.8, 126.0, 125.7, 125.6, 120.9, 48.4, 46.8, 36.1, 27.7, 25.1; HRMS (EI) m/z: Calcd for $\mathrm{C}_{29} \mathrm{H}_{25} \mathrm{NO}$ 403.1936; Found 403.1938. HPLC (Chiralpak AD-3, $i$ $\mathrm{PrOH} /$ hexane $=20 / 80$, flow rate $=1.0 \mathrm{~mL} / \mathrm{min}, \lambda=254 \mathrm{~nm}): \mathrm{t}_{\text {minor }}=12.4, \mathrm{t}_{\text {major }}=13.2$, ee $=$ $97 \%$.

1 mmol scale for the synthesis of 3a and 3a': To a solution of 4-vinylquinoline 1a (165 mg, $1.0 \mathrm{mmol}$ ) and 4-phenylhexa-2,4-dienal $2 \mathbf{a}(345 \mathrm{mg}, 2.0 \mathrm{mmol})$ in $\mathrm{CH}_{2} \mathrm{Cl}_{2}(4.0 \mathrm{~mL})$ was added catalyst $\mathbf{I}(65 \mathrm{mg}, 0.2 \mathrm{mmol})$ and $\mathrm{CH}_{3} \mathrm{SO}_{3} \mathrm{H}(48 \mathrm{mg}, 0.5 \mathrm{mmol})$. The resulting solution was stirred for $72 \mathrm{~h}$ at room temperature. The crude reaction mixture was directly purified by column chromatography on silica gel $(\mathrm{PE}: \mathrm{EA}=1: 2)$ to give the corresponding product in total $92 \%$ yield $(300 \mathrm{mg})\left(\mathbf{3 a}: \mathbf{3 a} \mathbf{a}^{\prime}=41: 51\right)$.

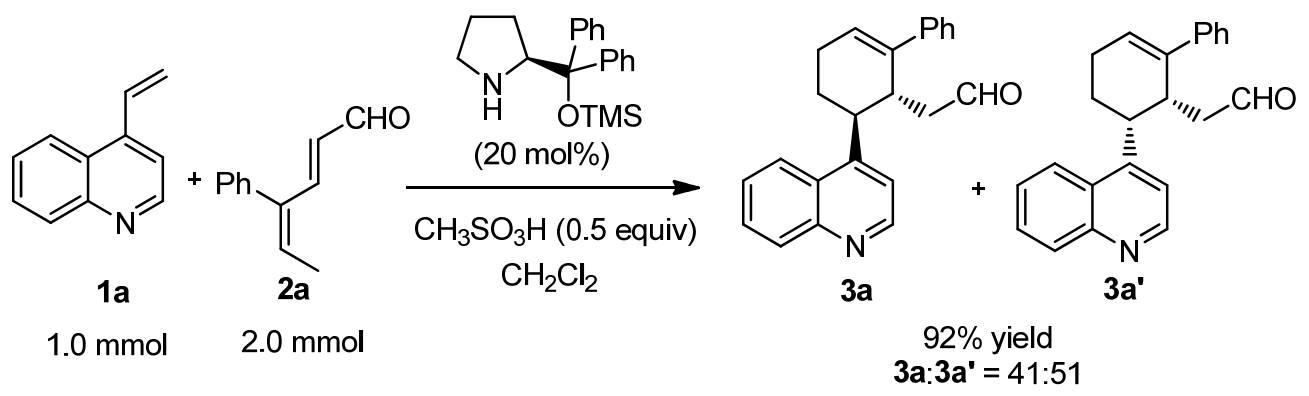




\section{X-ray crystal structures for compounds 6 and $3 e^{\text {' }}$}

The sample 3e was hard to recrystallize. Herein it was reduced by $\mathrm{NaBH}_{4}$ in $\mathrm{MeOH}$ to get compound $\mathbf{6}$. The purified compound $\mathbf{6}$ was recrystallized in $\mathrm{CDCl}_{3} / \mathrm{Et}_{2} \mathrm{O}$ solution to get crystal for single crystal X-ray analysis.

Data collection: Bruker APEX2; cell refinement: Bruker SAINT; data reduction: Bruker SAINT; program(s) used to solve structure: SHELXS97 (Sheldrick, 2008); program(s) used to refine structure: SHELXL97 (Sheldrick, 2008); molecular graphics: Bruker SHELXTL; software used to prepare material for publication: Bruker SHELXTL.

Crystal data and structure refinement of compound $6($ Prob $=50)$.

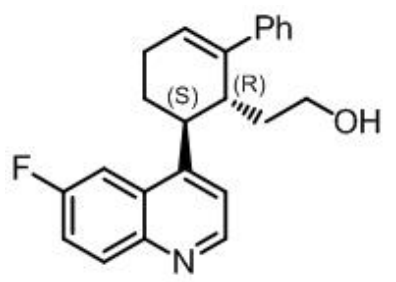

6

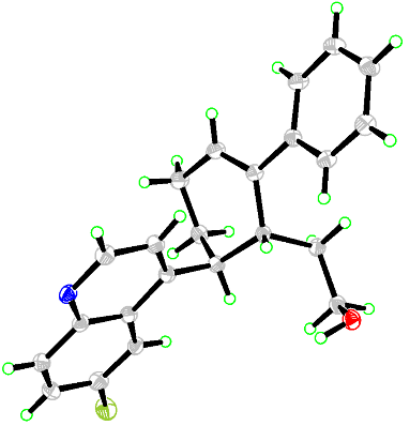

Crystal data

\begin{tabular}{|c|c|}
\hline $\mathrm{C}_{46} \mathrm{H}_{46} \mathrm{~F}_{2} \mathrm{~N}_{2} \mathrm{O}_{3}$ & $F(000)=1496$ \\
\hline$M_{r}=712.85$ & $D_{\mathrm{x}}=1.297 \mathrm{Mg} \mathrm{m}^{-3}$ \\
\hline Orthorhombic, $P 2_{1} 2_{1} 2_{1}$ & $\mathrm{Cu} K \alpha$ radiation, $\lambda=1.54178 \AA$ \\
\hline$a=9.3260(2) \AA$ & $\mu=0.68 \mathrm{~mm}^{-1}$ \\
\hline$b=15.1974(3) \AA$ & $T=296 \mathrm{~K}$ \\
\hline$c=25.7548(5) \AA$ & Block, colorless \\
\hline$V=3650.25(13) \quad \AA^{3}$ & $0.15 \times 0.10 \times 0.10 \mathrm{~mm}$ \\
\hline
\end{tabular}

Data collection

\begin{tabular}{|c|c|}
\hline Bruker APEX-II CCD diffractometer & 6161 independent reflections \\
\hline Radiation source: fine-focus sealed tube & 5598 reflections with $I>2 \sigma(I)$ \\
\hline graphite & $R_{\text {int }}=0.104$ \\
\hline phi and $\omega$ scans & $\theta_{\max }=64.8^{\circ}, \theta_{\min }=3.4^{\circ}$ \\
\hline Absorption correction: multi-scan $S A D A B S$ & $h=-10 \rightarrow 10$ \\
\hline$T_{\min }=0.968, T_{\max }=0.989$ & $k=-17 \rightarrow 17$ \\
\hline 115849 measured reflections & $l=-30 \rightarrow 30$ \\
\hline
\end{tabular}

Refinement

\begin{tabular}{|c|c|}
\hline Refinement on $F^{2}$ & Hydrogen site location: inferred from neighbouring sites \\
\hline Least-squares matrix: full & $\begin{array}{c}\mathrm{H} \text { atoms treated by a mixture of independent and } \\
\text { constrained refinement }\end{array}$ \\
\hline$R\left[F^{2}>2 \sigma\left(F^{2}\right)\right]=0.049$ & $w=1 /\left[\sigma^{2}\left(F_{\mathrm{o}}^{2}\right)+(0.0597 P)^{2}+2.5299 P\right]$ where $P=\left(F_{\mathrm{o}}^{2}+\right.$ \\
$\left.2 F_{\mathrm{c}}{ }^{2}\right) / 3$
\end{tabular}




\begin{tabular}{|c|c|}
\hline 491 parameters & $\begin{array}{c}\text { Extinction correction: SHELXL, } \\
\mathrm{Fc}^{*}=\mathrm{kFc}\left[1+0.001 \mathrm{xFc}^{2} \lambda^{3} / \mathrm{sin}(2 \theta)\right]^{-1 / 4}\end{array}$ \\
\hline 3 restraints & Extinction coefficient: 0.00051 (10) \\
\hline $\begin{array}{c}\text { Primary atom site location: structure-invariant } \\
\text { direct methods }\end{array}$ & $\begin{array}{c}\text { Absolute structure: Flack H D (1983), Acta Cryst. A39, } \\
\text { Fecondary atom site location: difference } \\
\text { Fourier map }\end{array}$ \\
\hline \begin{tabular}{c} 
Seck parameter: 0.03 (19) \\
\hline
\end{tabular} & \\
\hline
\end{tabular}

Special details

Geometry. All esds (except the esd in the dihedral angle between two 1.s. planes) are estimated using the full covariance matrix. The cell esds are taken into account individually in the estimation of esds in distances, angles and torsion angles; correlations between esds in cell parameters are only used when they are defined by crystal symmetry. An approximate (isotropic) treatment of cell esds is used for estimating esds involving 1.s. planes.

Refinement. Refinement of $\mathrm{F}^{2}$ against ALL reflections. The weighted R-factor $\mathrm{wR}$ and goodness of fit $\mathrm{S}$ are based on $\mathrm{F}^{2}$, conventional $\mathrm{R}$-factors $\mathrm{R}$ are based on $\mathrm{F}$, with $\mathrm{F}$ set to zero for negative $\mathrm{F}^{2}$. The threshold expression of $\mathrm{F}^{2}>2$ sigma $\left(\mathrm{F}^{2}\right)$ is used only for calculating R-factors (gt) etc. and is not relevant to the choice of reflections for refinement. R-factors based on $\mathrm{F}^{2}$ are statistically about twice as large as those based on F, and R- factors based on ALL data will be even larger.

Crystal data and structure refinement of $3 \mathbf{e}^{\prime}(\mathrm{Prob}=50)$.

The sample 3e' was recrystallized in $\mathrm{CDCl}_{3} / \mathrm{Et}_{2} \mathrm{O}$ solution to get the crystal for single crystal X-ray analysis.
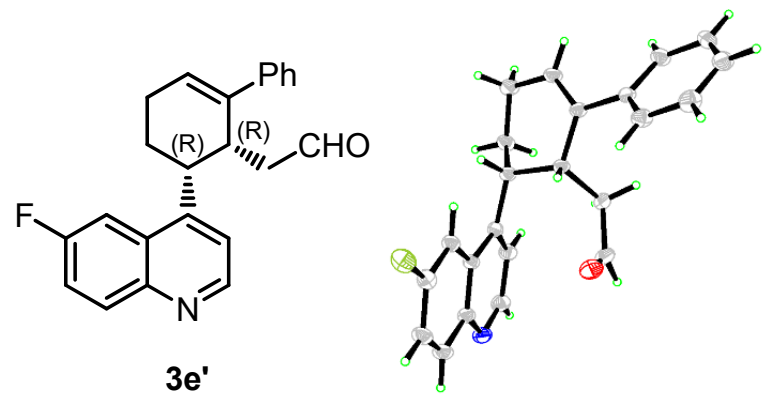

Crystal data

\begin{tabular}{|c|c|}
\hline $\mathrm{C}_{23} \mathrm{H}_{20} \mathrm{FNO}$ & $Z=2$ \\
\hline$M_{r}=345.40$ & $F(000)=366$ \\
\hline Monoclinic, $P 2_{1}$ & $D_{\mathrm{x}}=1.295 \mathrm{Mg} \mathrm{m}^{-3}$ \\
\hline$a=6.7007(19) \AA$ & $\mathrm{Cu} K \alpha$ radiation, $\lambda=1.54178 \AA$ \\
\hline$b=7.379(2) \AA$ & $\mu=0.69 \mathrm{~mm}^{-1}$ \\
\hline$c=17.978(5) \AA$ & $T=296 \mathrm{~K}$ \\
\hline$\beta=92.104(17)^{\circ}$ & Block, colorless \\
\hline$V=888.3(4) \AA^{3}$ & $0.15 \times 0.10 \times 0.10 \mathrm{~mm}$ \\
\hline
\end{tabular}

Data collection

\begin{tabular}{|c|c|}
\hline Bruker APEX-II CCD diffractometer & 2962 independent reflections \\
\hline Radiation source: fine-focus sealed tube & 2372 reflections with $I>2 \sigma(I)$ \\
\hline graphite & $R_{\text {int }}=0.076$ \\
\hline
\end{tabular}




\begin{tabular}{|c|c|}
\hline phi and $\omega$ scans & $\Theta_{\max }=64.7^{\circ}, \Theta_{\min }=2.5^{\circ}$ \\
\hline Absorption correction: multi-scan $S A D A B S$ & $h=-7 \rightarrow 7$ \\
\hline$T_{\min }=0.968, T_{\max }=0.989$ & $k=-8 \rightarrow 8$ \\
\hline 12982 measured reflections & $l=-21 \rightarrow 20$ \\
\hline
\end{tabular}

Refinement
\begin{tabular}{|c|c|}
\hline Refinement on $F^{2}$ & Hydrogen site location: inferred from neighbouring sites \\
\hline Least-squares matrix: full & $\begin{array}{c}\mathrm{H} \text { atoms treated by a mixture of independent and } \\
\text { constrained refinement }\end{array}$ \\
\hline$R\left[F^{2}>2 \sigma\left(F^{2}\right)\right]=0.038$ & $\begin{array}{c}w=1 /\left[\sigma^{2}\left(F_{\mathrm{o}}{ }^{2}\right)+(0.0702 P)^{2}\right] \\
\text { where } P=\left(F_{\mathrm{o}}{ }^{2}+2 F_{\mathrm{c}}{ }^{2}\right) / 3\end{array}$ \\
\hline$w R\left(F^{2}\right)=0.124$ & $(\Delta / \sigma)_{\max }<0.001$ \\
\hline$S=1.12$ & $\Delta\rangle_{\max }=0.19 \mathrm{e} \AA^{-3}$ \\
\hline 2962 reflections & $\Delta\rangle_{\min }=-0.18 \mathrm{e} \AA^{-3}$ \\
\hline 236 parameters & $\begin{array}{c}\text { Extinction correction: } S H E L X L, \\
\mathrm{Fc}^{*}=\mathrm{kFc}\left[1+0.001 \mathrm{xFc} \mathrm{c}^{2} / \mathrm{sin}(2 \Theta)\right]^{-1 / 4}\end{array}$ \\
\hline 1 restraint & Extinction coefficient: $0.0040(12)$ \\
\hline $\begin{array}{c}\text { Primary atom site location: structure-invariant } \\
\text { direct methods }\end{array}$ & $\begin{array}{c}\text { Absolute structure: Flack H D }(1983), \text { Acta Cryst. A39, } \\
876-881\end{array}$ \\
\hline Secondary atom site location: difference & Flack parameter: $0.1(2)$ \\
\hline
\end{tabular}

Special details

Geometry. All esds (except the esd in the dihedral angle between two 1.s. planes) are estimated using the full covariance matrix. The cell esds are taken into account individually in the estimation of esds in distances, angles and torsion angles; correlations between esds in cell parameters are only used when they are defined by crystal symmetry. An approximate (isotropic) treatment of cell esds is used for estimating esds involving 1.s. planes.

Refinement. Refinement of $\mathrm{F}^{2}$ against ALL reflections. The weighted R-factor $w \mathrm{R}$ and goodness of fit $\mathrm{S}$ are based on $\mathrm{F}^{2}$, conventional $\mathrm{R}$-factors $\mathrm{R}$ are based on $\mathrm{F}$, with $\mathrm{F}$ set to zero for negative $F^{2}$. The threshold expression of $F^{2}>2 \operatorname{sigma}\left(F^{2}\right)$ is used only for calculating $R$ factors (gt) etc. and is not relevant to the choice of reflections for refinement. R-factors based on $\mathrm{F}^{2}$ are statistically about twice as large as those based on $\mathrm{F}$, and $\mathrm{R}$ - factors based on ALL data will be even larger.

\section{References:}

1. S. Wang, X. Li, H. Liu, L. Xu, J. Zhuang, J. Li, H. Li, W. Wang, J. Am. Chem. Soc. 2015, 137, 2303.

2. J. G. Rodriguez, Y. Benito, J. Heterocyclic Chem. 1988, 25(3), 819. 
VI. ${ }^{1} \mathrm{H}$ NMR and ${ }^{13} \mathrm{C}$ NMR spectra

7-Chloro-4-vinylquinoline $\left[{ }^{1} \mathrm{H} \_\mathrm{NMR} \_400 \mathrm{MHz}\left(\mathrm{CDCl}_{3}: 7.26 \mathrm{ppm}\right)\right]$

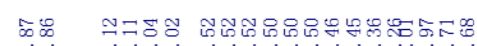

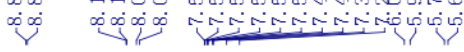

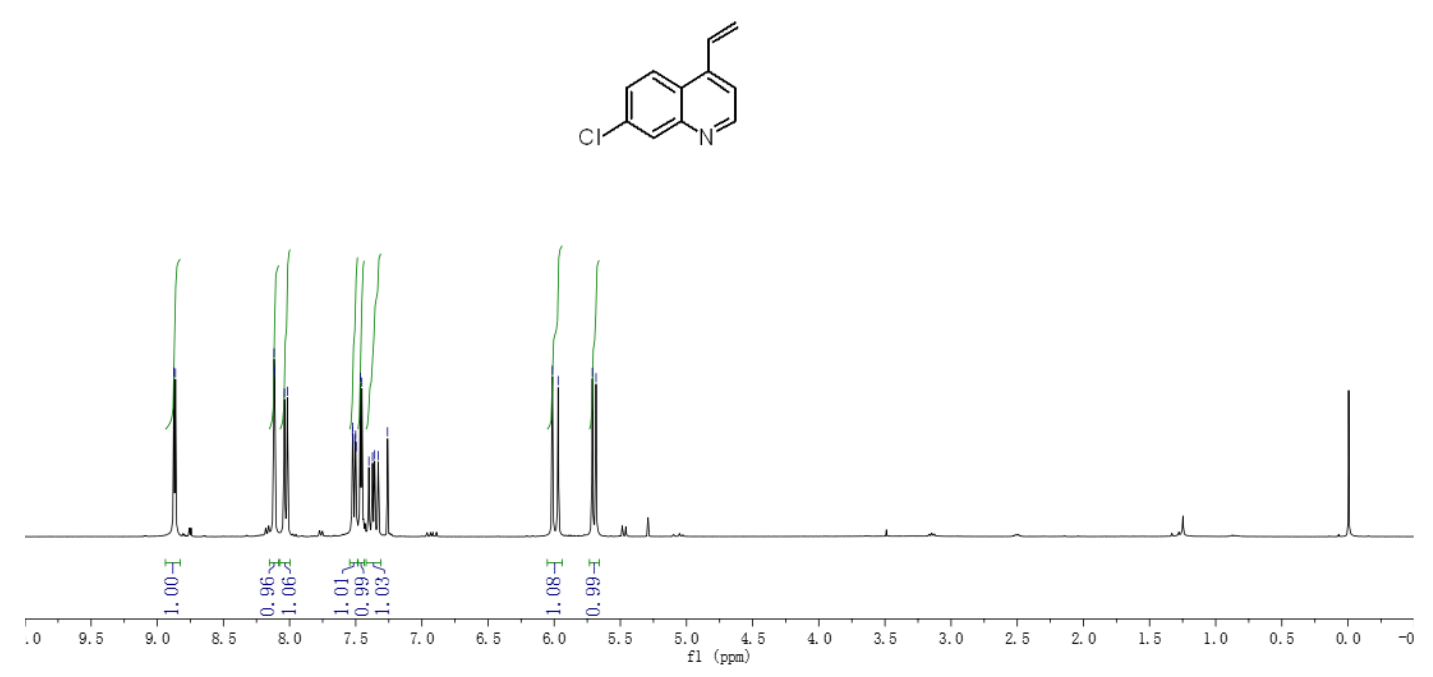

${ }^{13} \mathrm{C} \_N M R \_100 \mathrm{MHz}\left(\mathrm{CDCl}_{3}: 77.00 \mathrm{ppm}\right)$

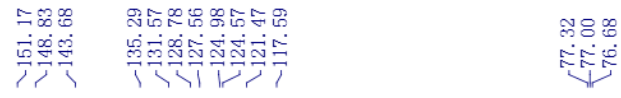
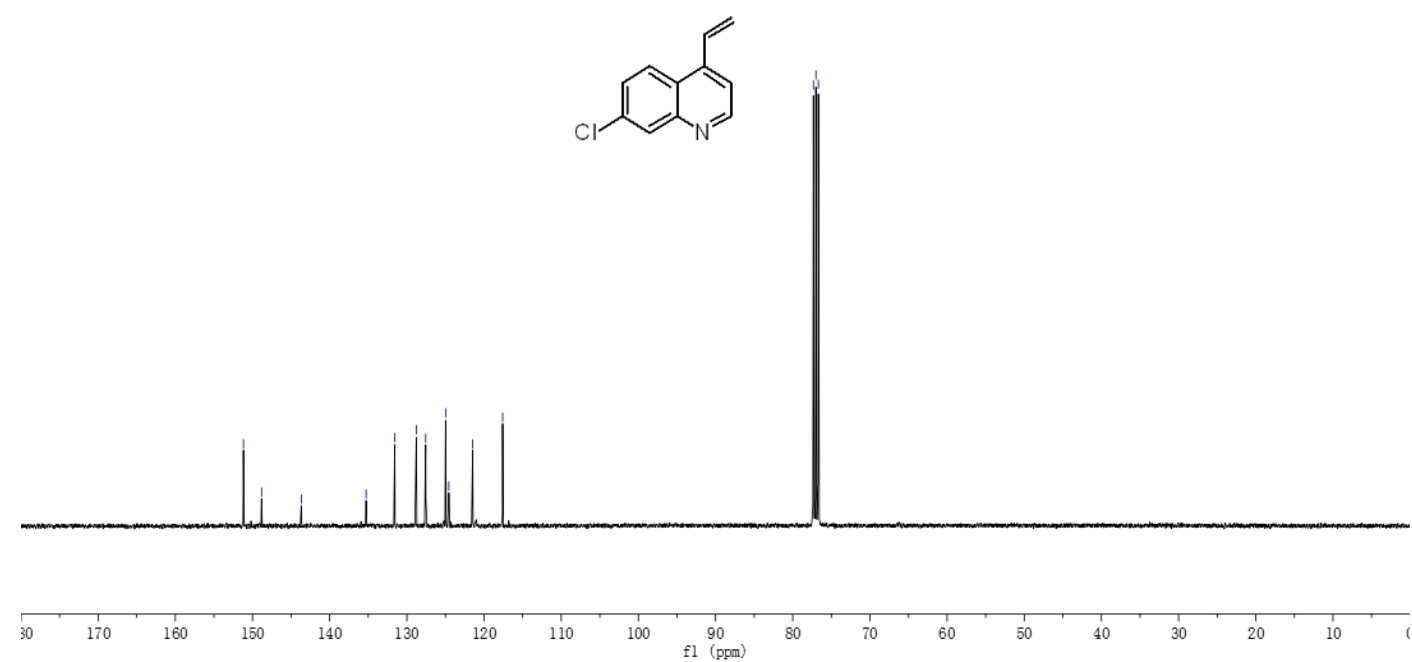
7-(Trifluoromethyl)-4-vinylquinoline [ $\left.{ }^{1} \mathrm{H} \_\mathrm{NMR} \_400 \mathrm{MHz}\left(\mathrm{CDCl}_{3}: 7.26 \mathrm{ppm}\right)\right]$

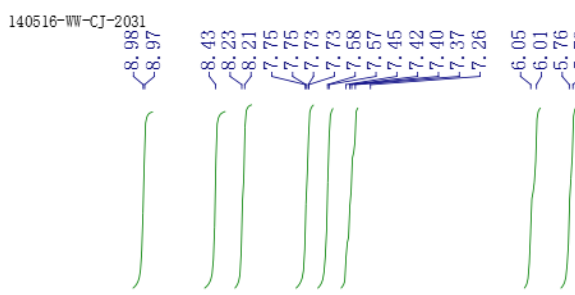<smiles>C=Cc1ccnc2cc(C(F)(F)F)ccc12</smiles>

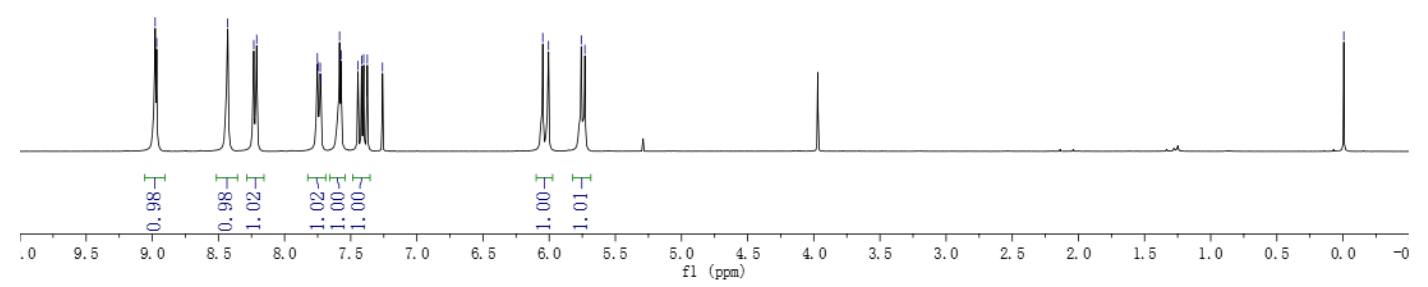

${ }^{13} \mathrm{C} \_N M R \_100 \mathrm{MHz}\left(\mathrm{CDCl}_{3}: 77.00 \mathrm{ppm}\right)$

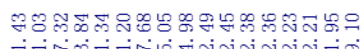

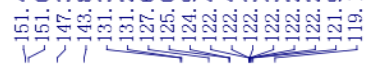
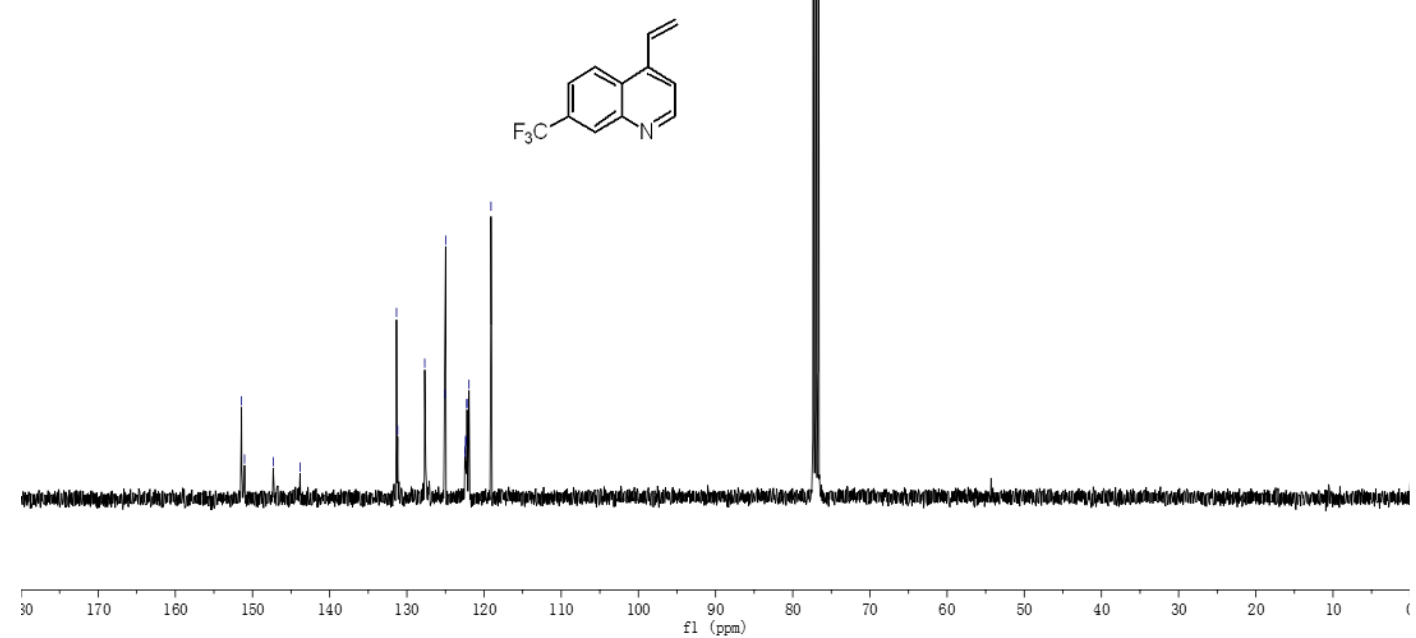
6-Fluoro-4-vinylquinoline $\left[{ }^{1} \mathrm{H}_{-} \mathrm{NMR} 4400 \mathrm{MHz}\left(\mathrm{CDCl}_{3}: 7.26 \mathrm{ppm}\right)\right]$
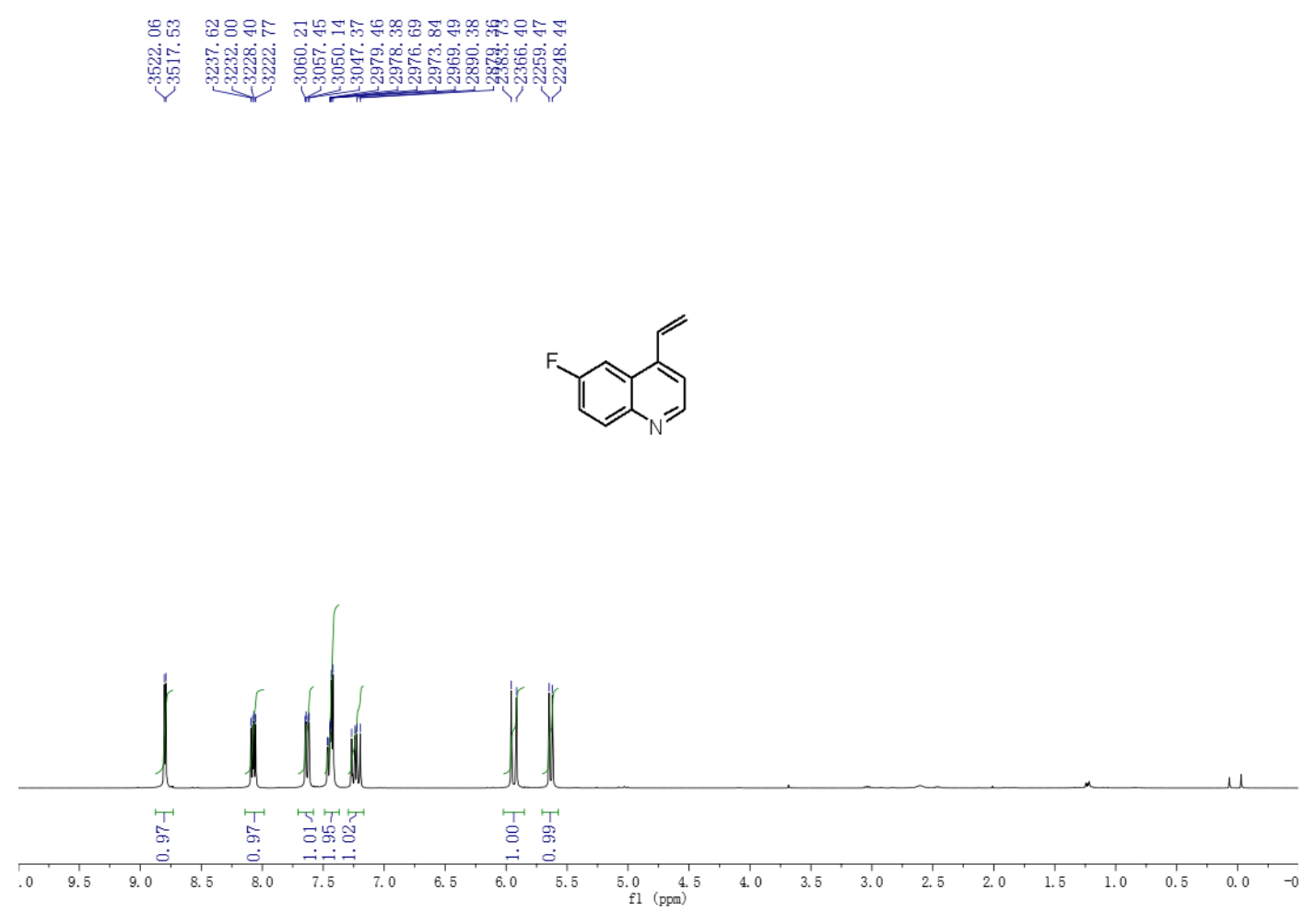

${ }^{13} \mathrm{C} \_\mathrm{NMR} \_100 \mathrm{MHz}\left(\mathrm{CDCl}_{3}: 77.00 \mathrm{ppm}\right)$
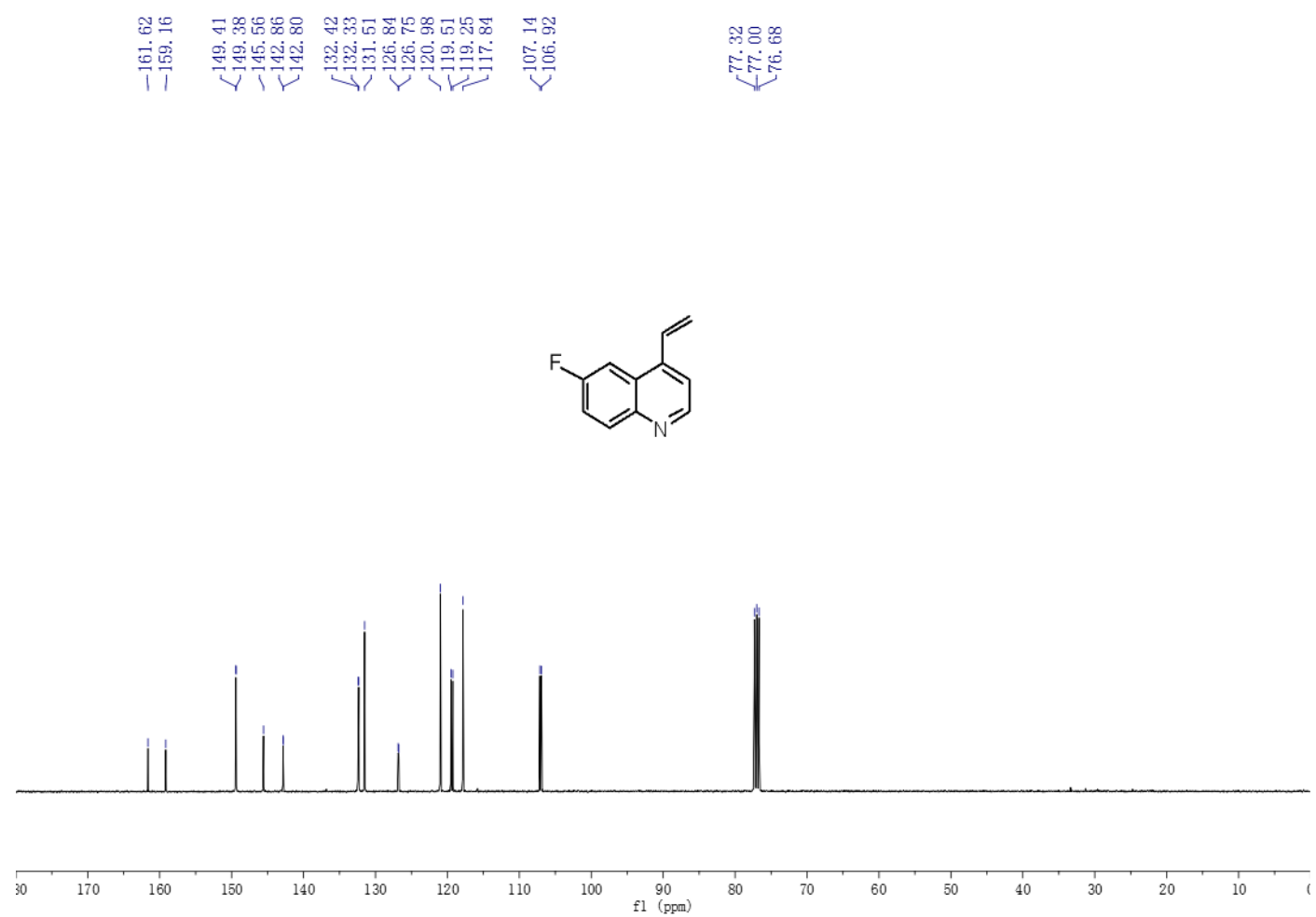
6-Methyl-4-vinylquinoline $\left[{ }^{1} \mathrm{H}_{-} \mathrm{NMR} \_400 \mathrm{MHz}\left(\mathrm{CDCl}_{3}: 7.26 \mathrm{ppm}\right)\right]$
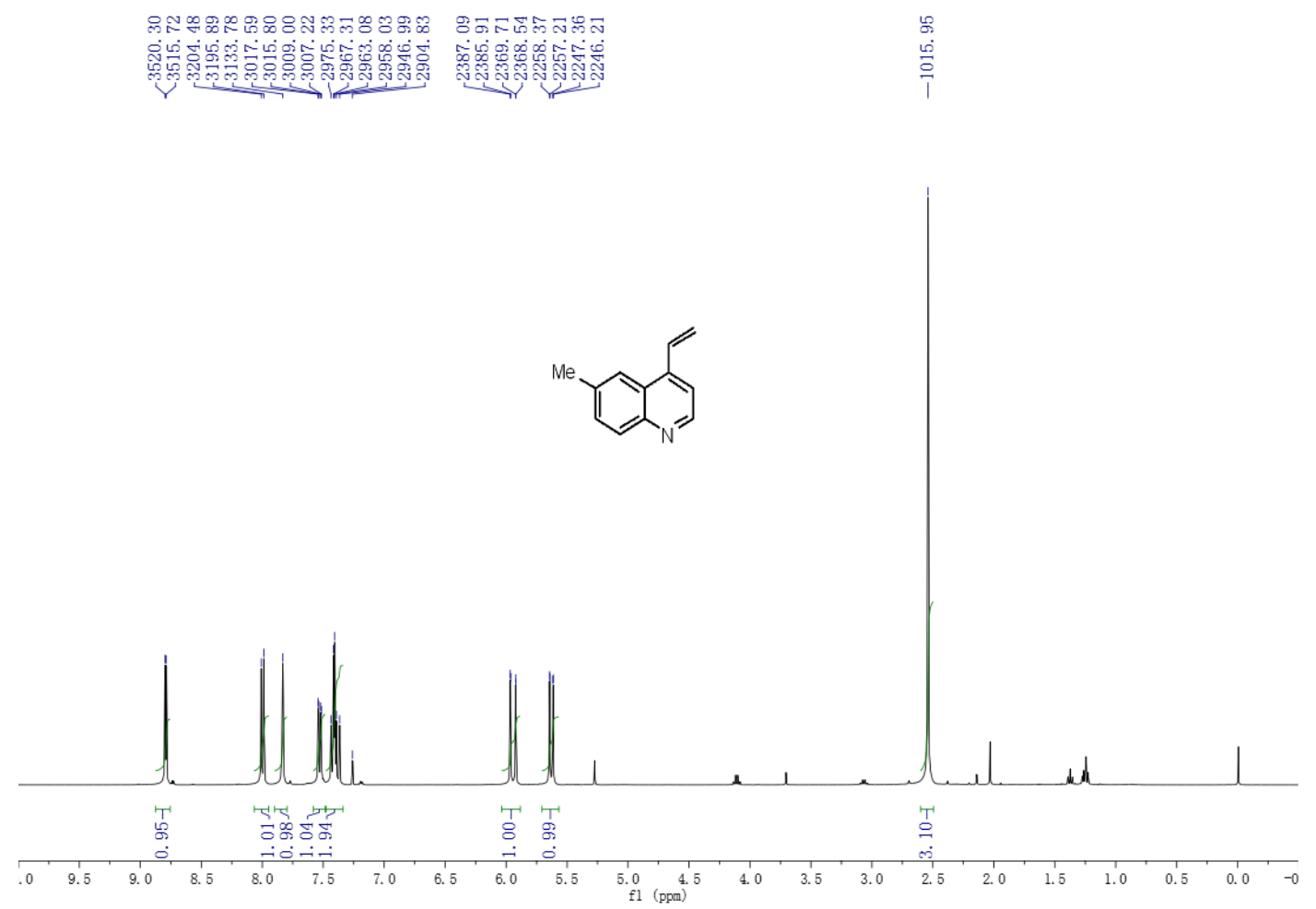

${ }^{13} \mathrm{C} \_N M R \_100 \mathrm{MHz}\left(\mathrm{CDCl}_{3}: 77.00 \mathrm{ppm}\right)$
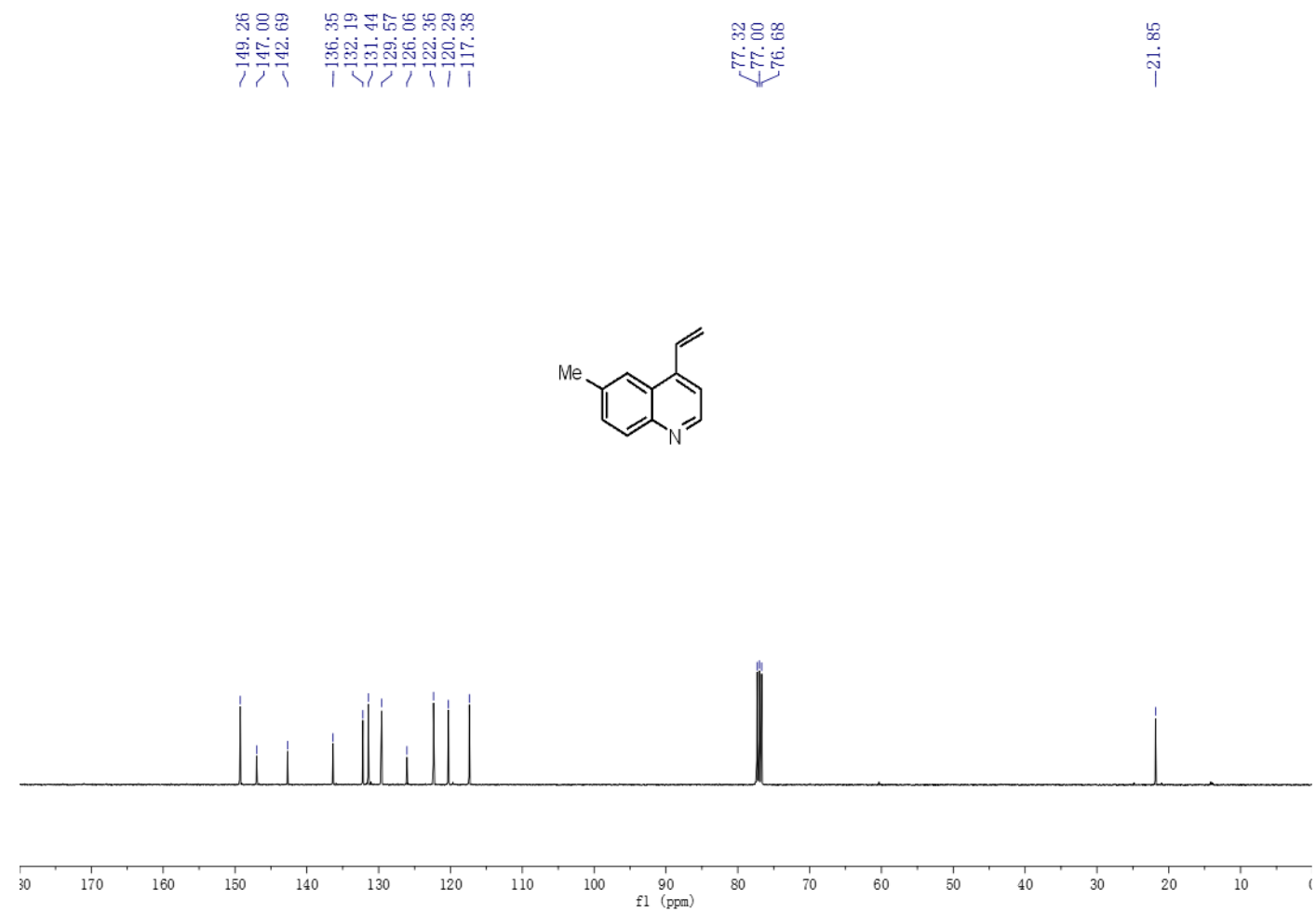
2-Methyl-4-vinylquinoline $\left[{ }^{1} \mathrm{H}_{-} \mathrm{NMR} \_400 \mathrm{MHz}\left(\mathrm{CDCl}_{3}: 7.26 \mathrm{ppm}\right)\right]$

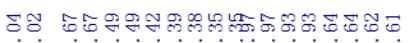

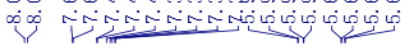

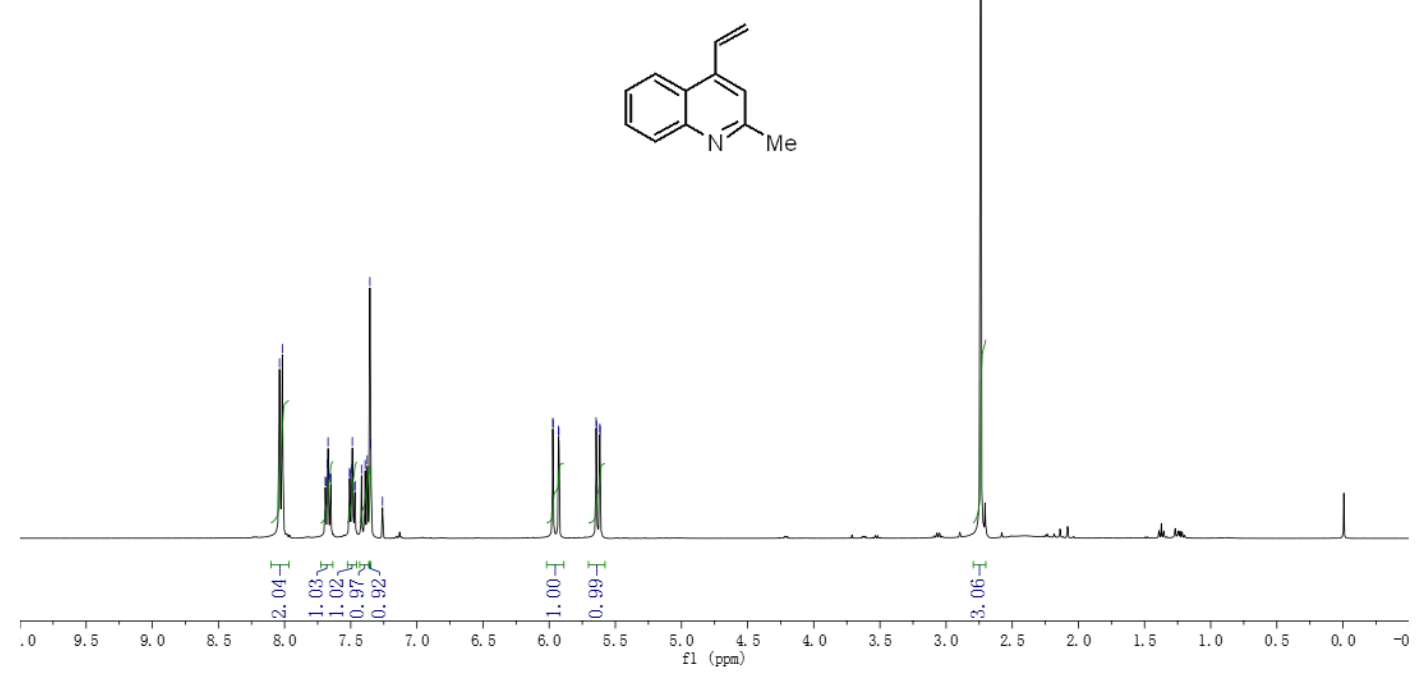

${ }^{13} \mathrm{C} \_\mathrm{NMR} \_100 \mathrm{MHz}\left(\mathrm{CDCl}_{3}: 77.00 \mathrm{ppm}\right)$
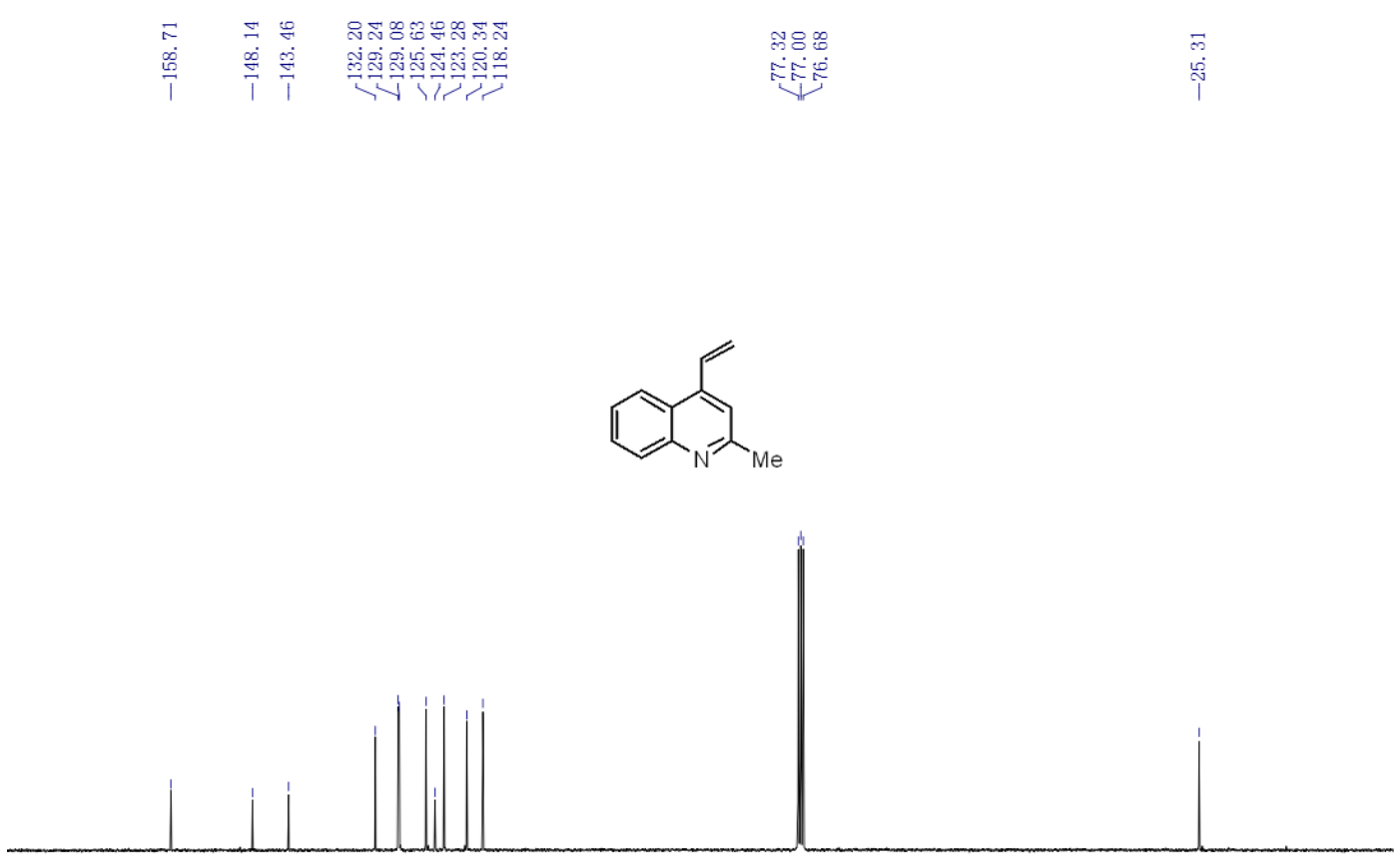

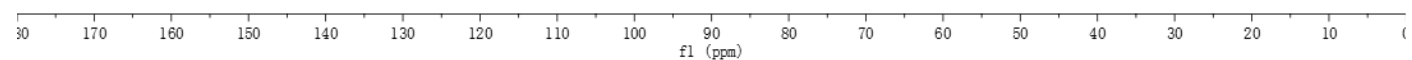


4-Phenyl-2-vinylquinoline $\left[{ }^{1} \mathrm{H}_{-} \mathrm{NMR} 4400 \mathrm{MHz}\left(\mathrm{CDCl}_{3}: 7.26 \mathrm{ppm}\right)\right]$

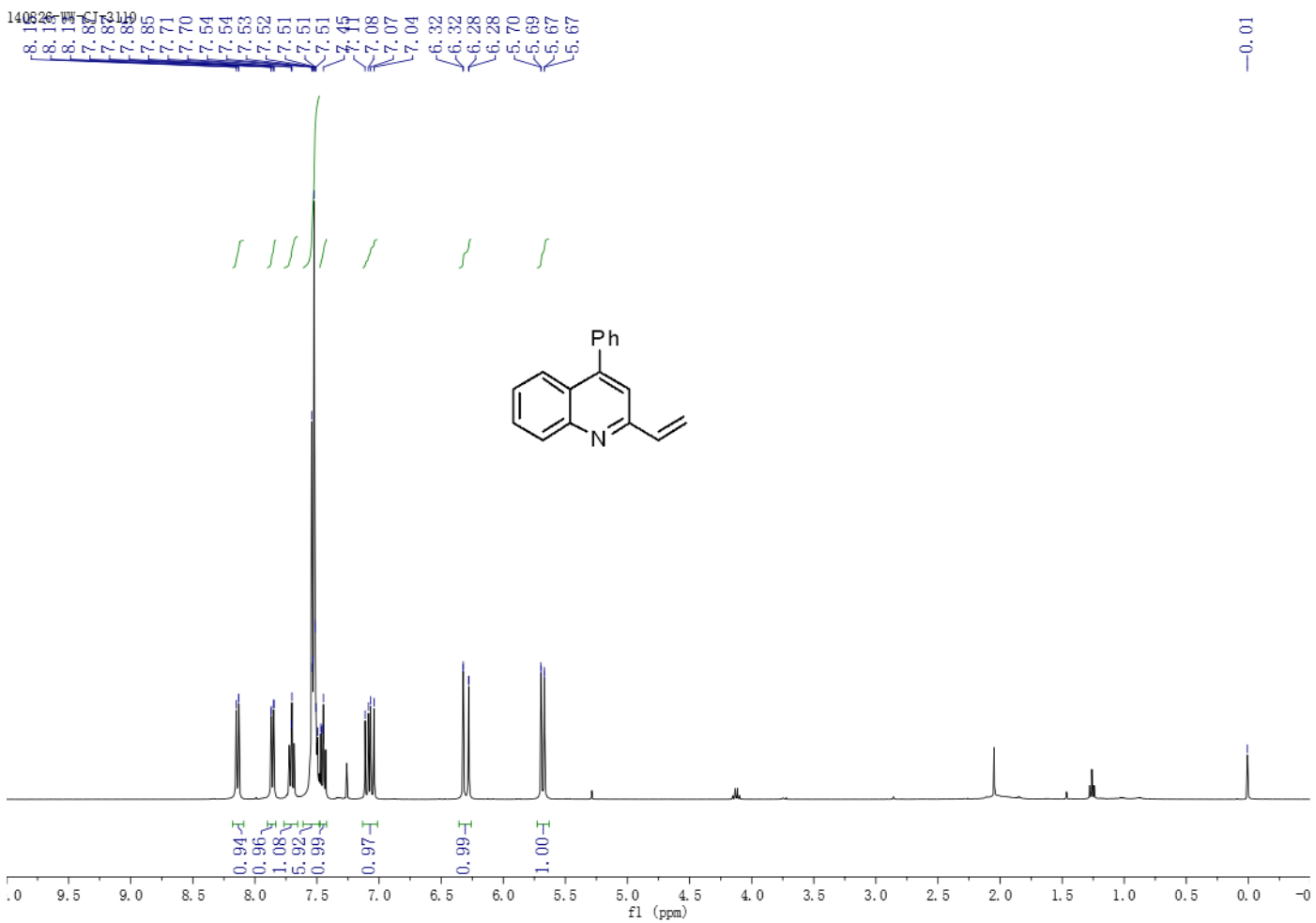

${ }^{13} \mathrm{C} \_\mathrm{NMR} \_100 \mathrm{MHz}\left(\mathrm{CDCl}_{3}: 77.00 \mathrm{ppm}\right)$

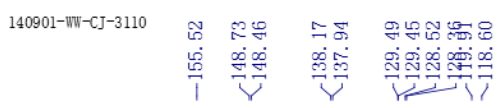

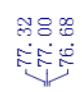

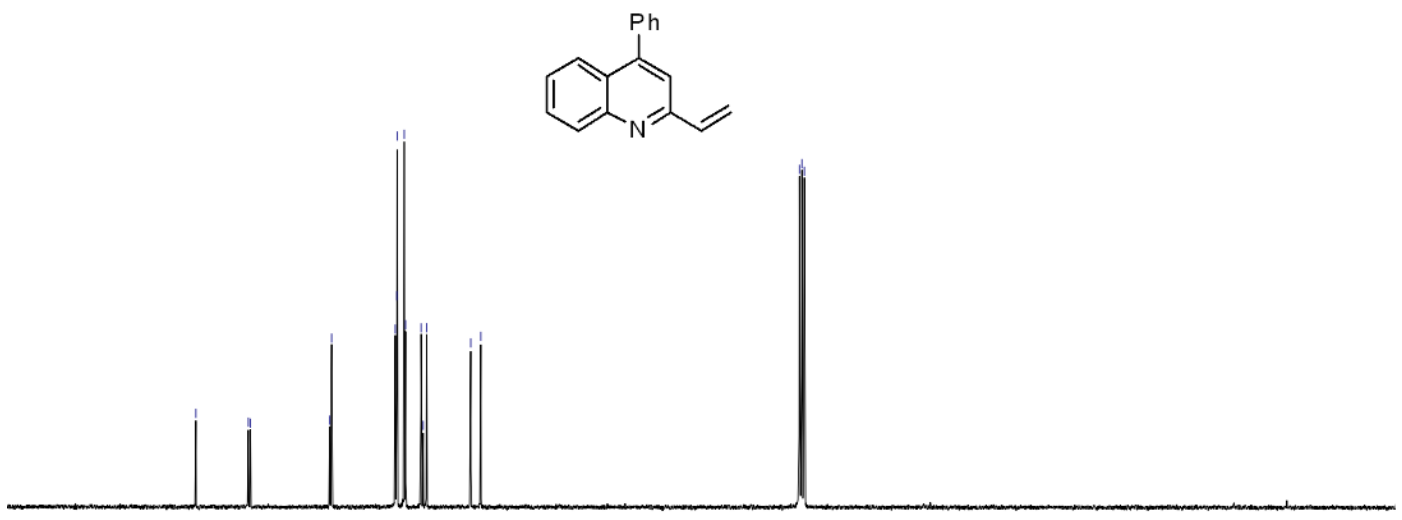

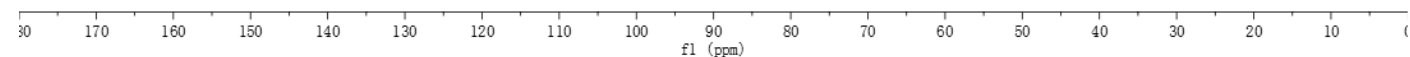




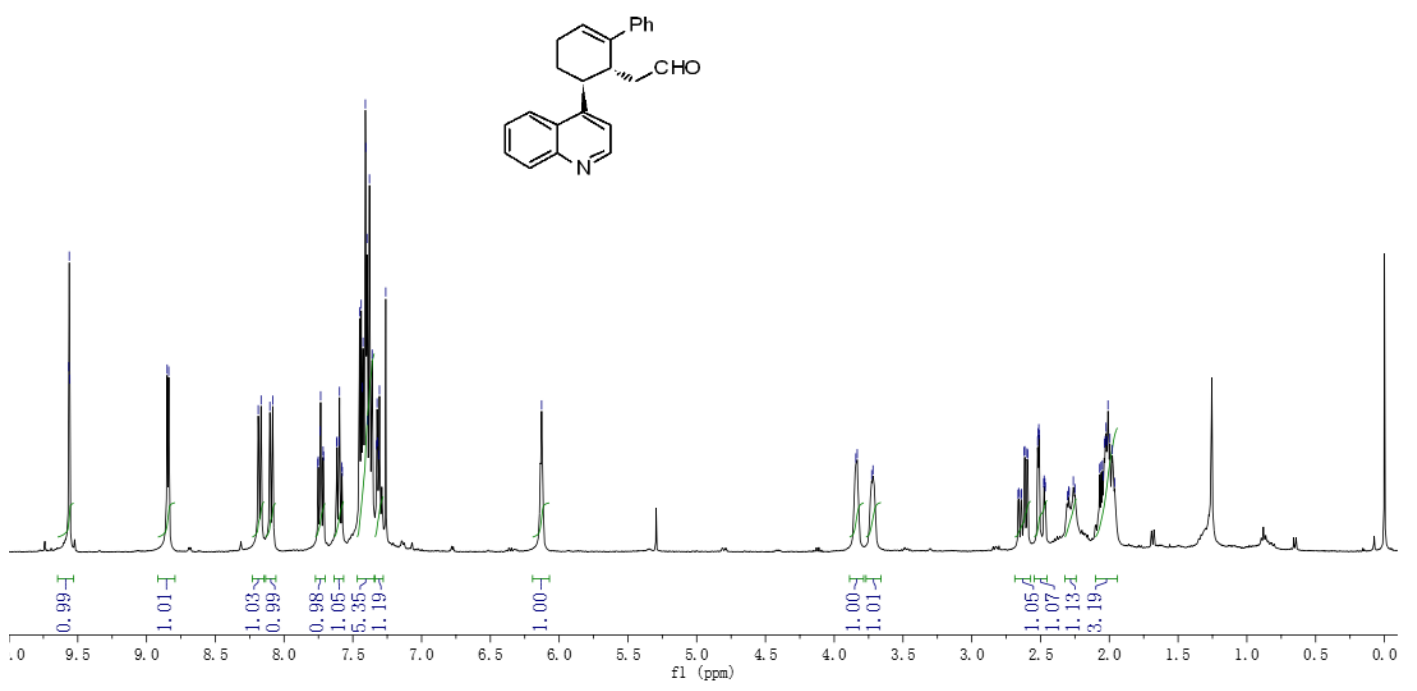

${ }^{13} \mathrm{C} \_\mathrm{NMR} \_100 \mathrm{MHz}\left(\mathrm{CDCl}_{3}: 77.00 \mathrm{ppm}\right)$

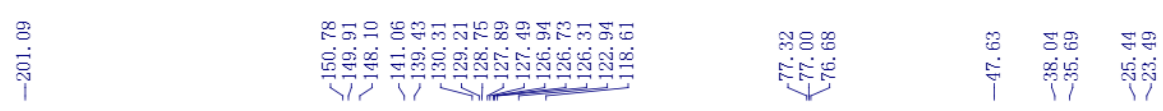
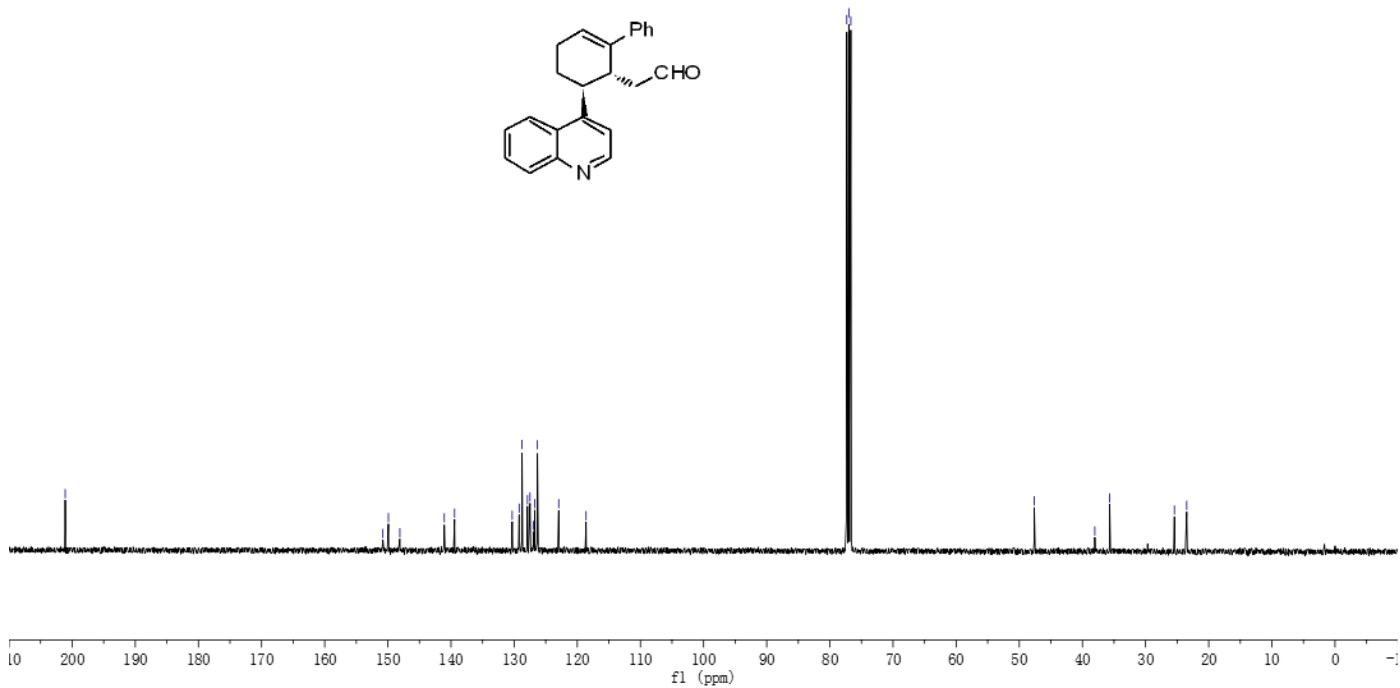
2-((2R,3R)-3-(Quinolin-4-yl)-2,3,4,5-tetrahydro-[1,1'-biphenyl]-2-yl)acetaldehyde (3a') $\left[{ }^{1} \mathrm{H} \_\mathrm{NMR} \_400 \mathrm{MHz} \_\left(\mathrm{CDCl}_{3}: 7.26 \mathrm{ppm}\right)\right]$

か

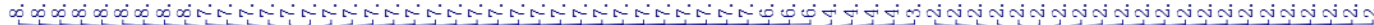

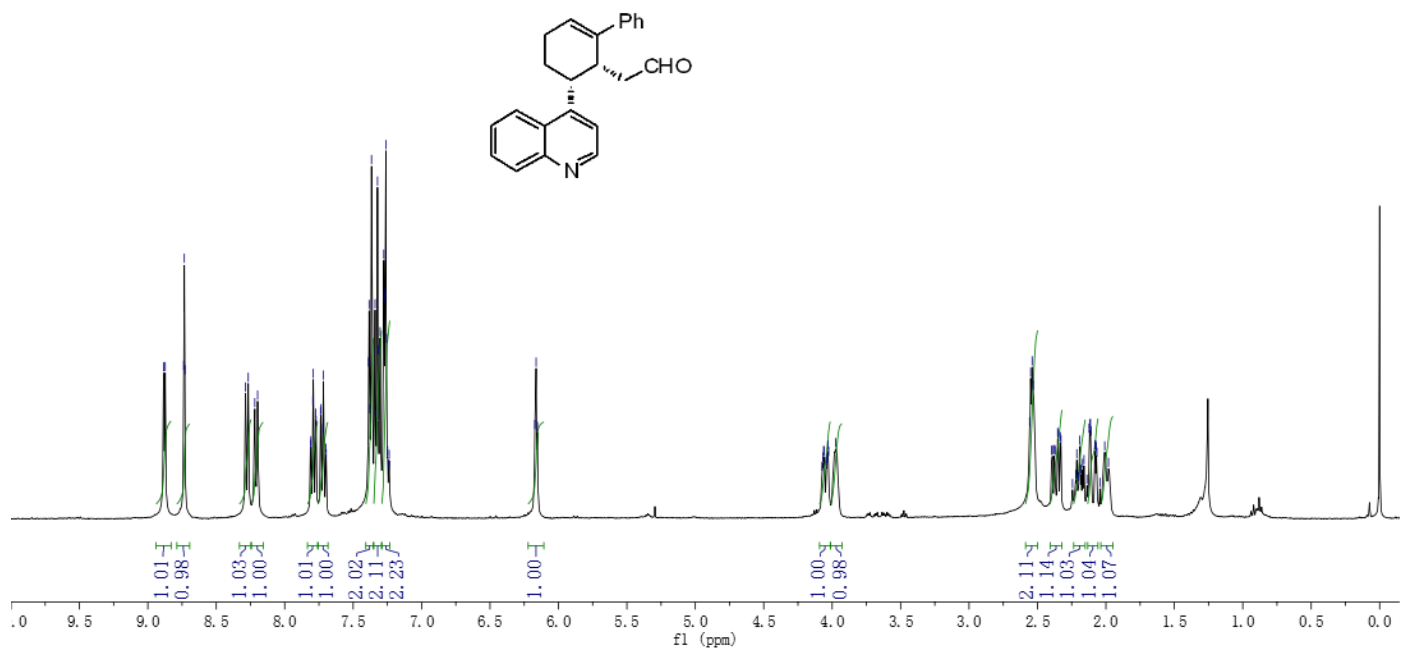

${ }^{13} \mathrm{C} \_\mathrm{NMR} \_100 \mathrm{MHz}\left(\mathrm{CDCl}_{3}: 77.00 \mathrm{ppm}\right)$

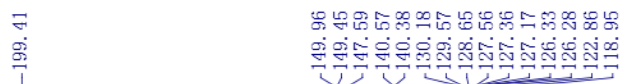

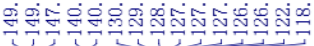
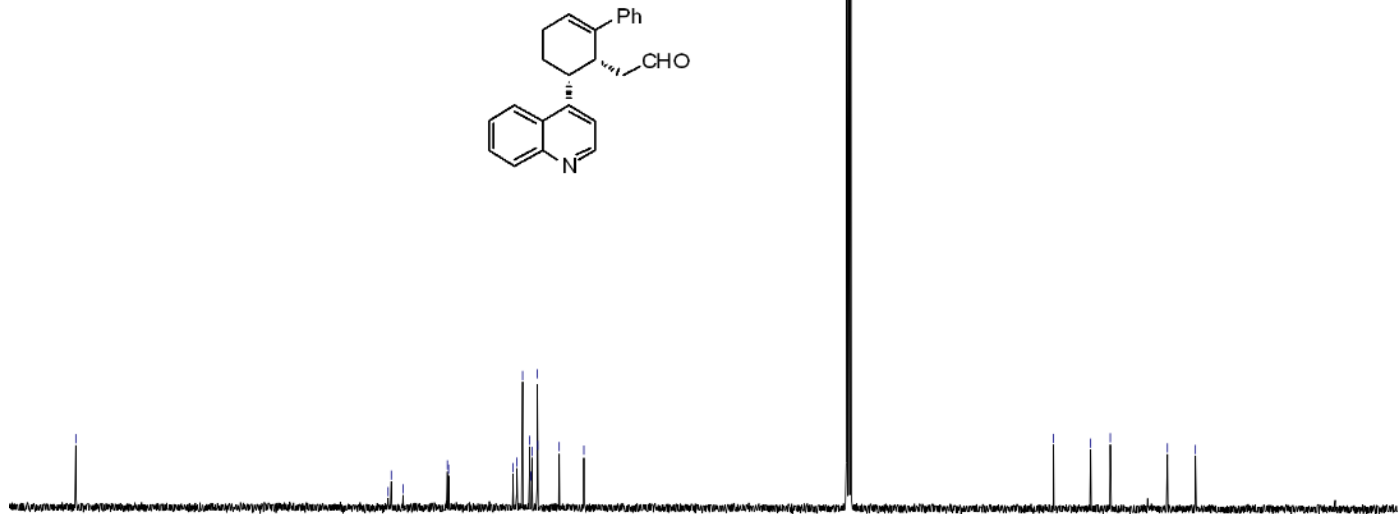

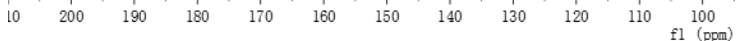




\section{2-((2R,3S)-3-(6-Methoxyquinolin-4-yl)-2,3,4,5-tetrahydro-[1,1'-biphenyl]-2-}

yl)acetaldehyde (3b) $\left[{ }^{1} \mathrm{H} \_\mathrm{NMR} \_400 \mathrm{MHz}\left(\mathrm{CDCl}_{3}: 7.26 \mathrm{ppm}\right)\right]$

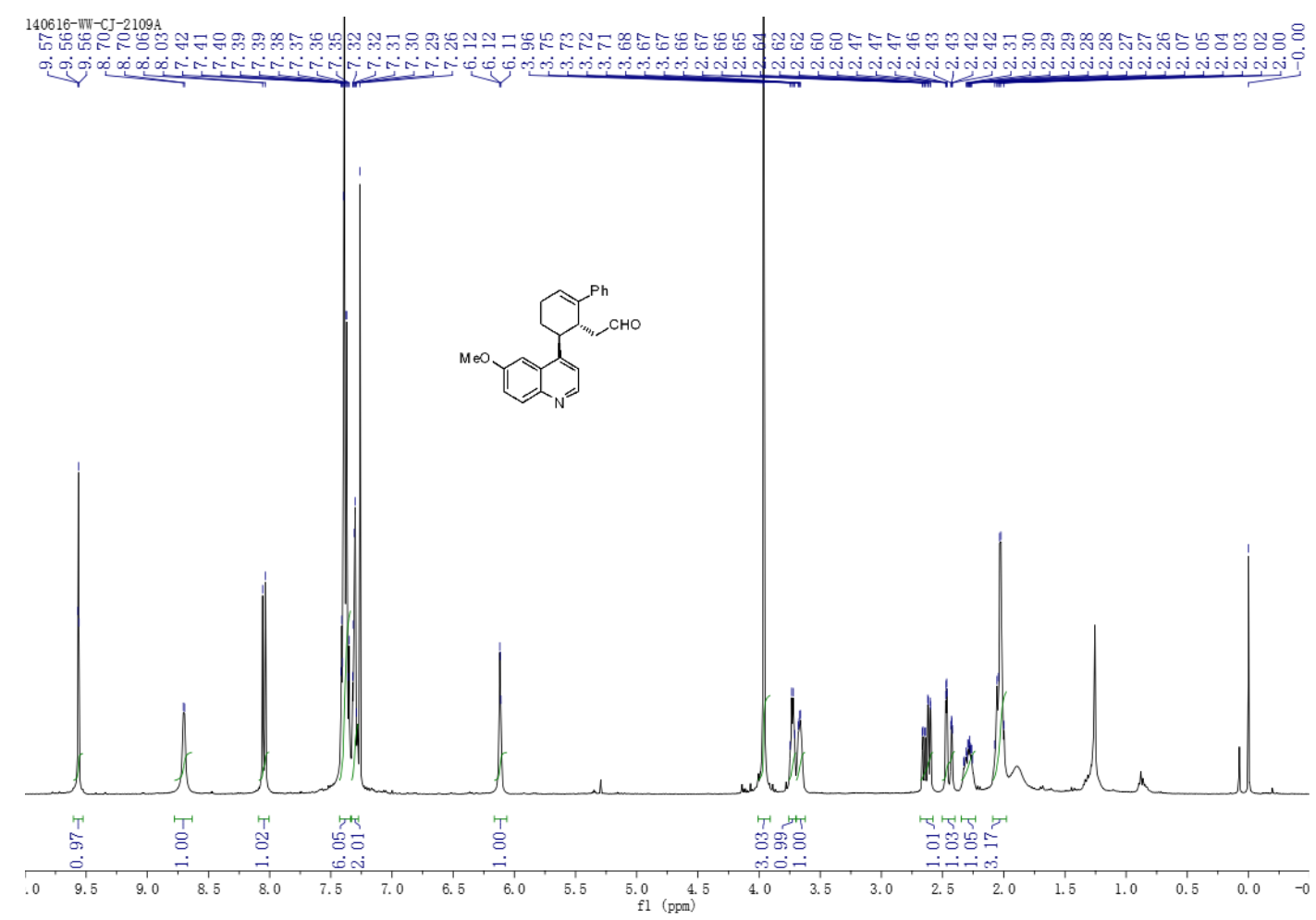

${ }^{13} \mathrm{C} \_N M R \_100 \mathrm{MHz}\left(\mathrm{CDCl}_{3}: 77.00 \mathrm{ppm}\right)$

\begin{tabular}{|c|c|c|c|c|c|c|c|}
\hline 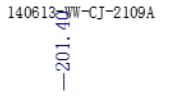 & $\begin{array}{l}\tilde{\infty} \\
\tilde{\omega} \\
\stackrel{1}{1}\end{array}$ & 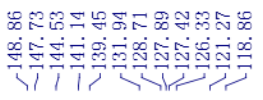 & 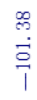 & 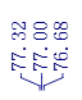 & $\begin{array}{l}\text { मी } \\
\text { ம் } \\
\text { | }\end{array}$ & $\stackrel{\oplus}{\stackrel{\oplus}{1}}$ & 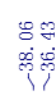 \\
\hline
\end{tabular}
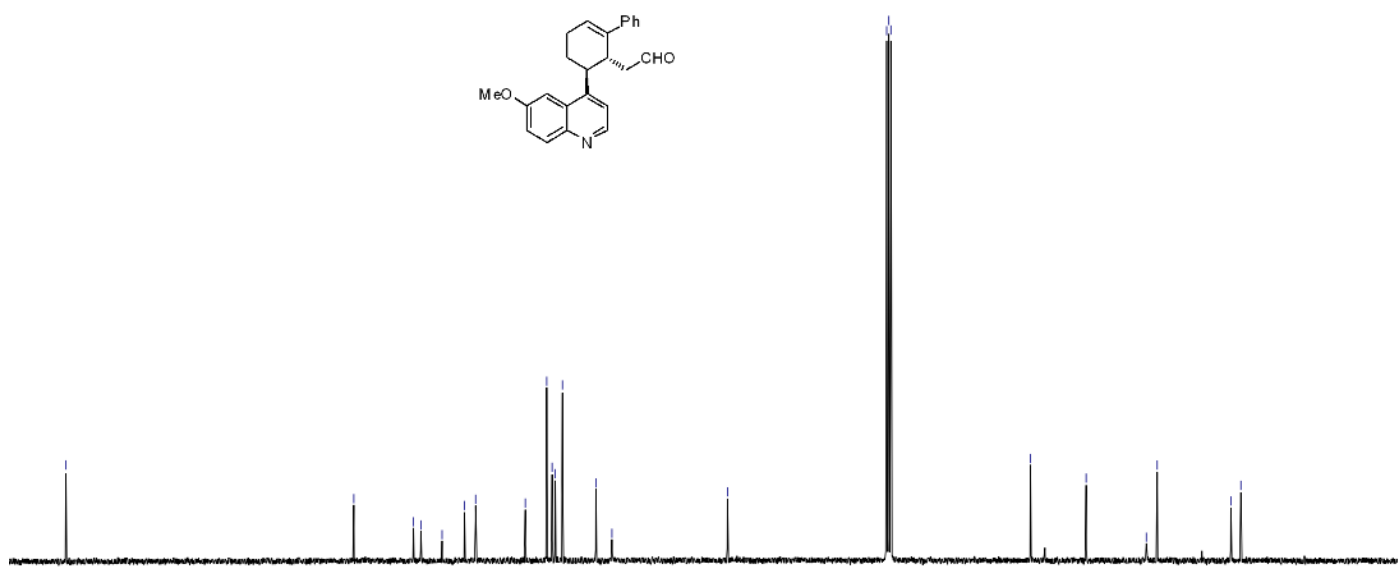

\begin{tabular}{lllllllllll}
\hline 10 & 200 & 190 & 180 & 170 & 160 & 150 & 140 & 130 & 120 & 110 \\
$\mathrm{fl} 1(\mathrm{ppm})$ & 100
\end{tabular} 
2-((2R,3R)-3-(6-Methoxyquinolin-4-yl)-2,3,4,5-tetrahydro-[1,1'-biphenyl]-2yl)acetaldehyde (3b') [ $\left.{ }^{1} \mathrm{H}_{-} \mathrm{NMR} \_400 \mathrm{MHz}\left(\mathrm{CDCl}_{3}: 7.26 \mathrm{ppm}\right)\right]$

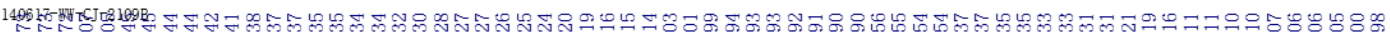

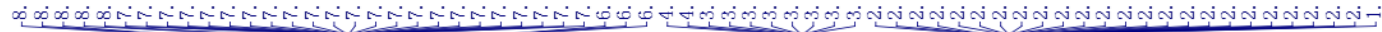

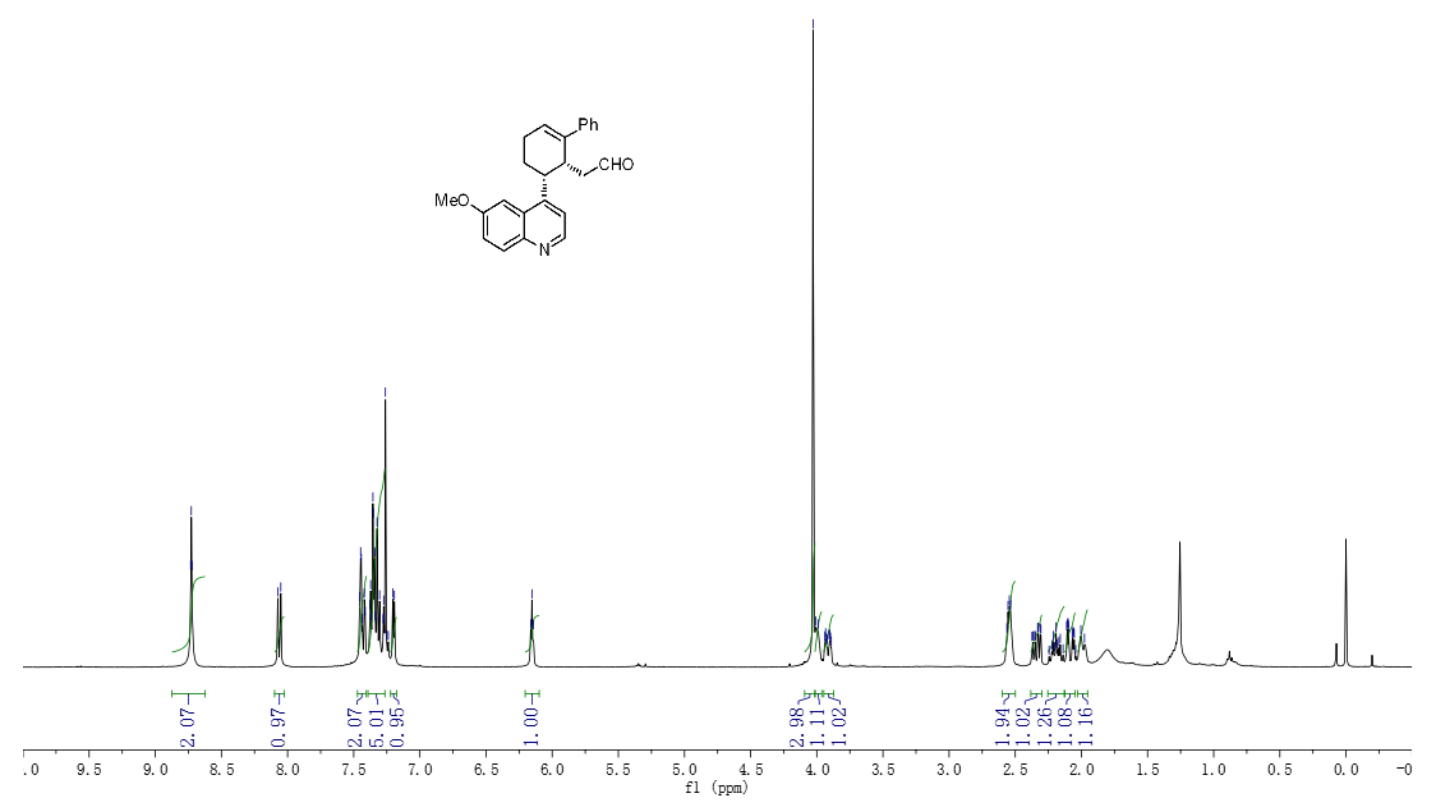

${ }^{13} \mathrm{C} \_$NMR_100 MHz_(CDCl $\left.3: 77.00 \mathrm{ppm}\right)$

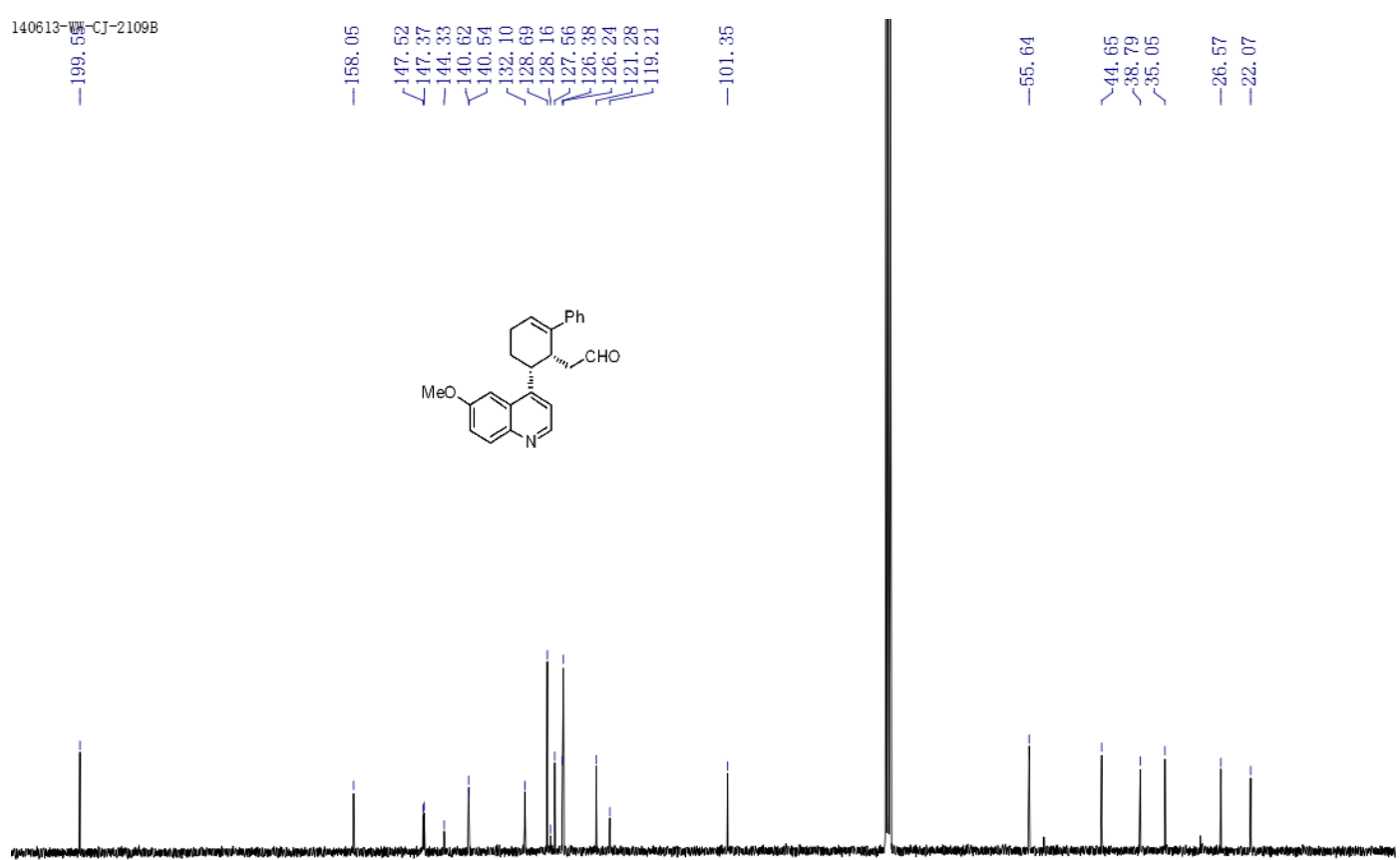

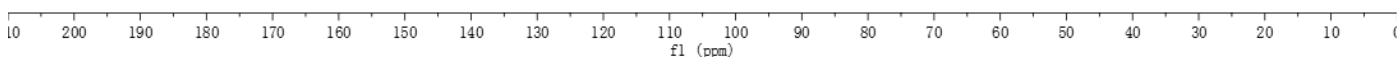


2-((2R,3S)-3-(7-Chloroquinolin-4-yl)-2,3,4,5-tetrahydro-[1,1'-biphenyl]-2yl)acetaldehyde (3c) $\left[{ }^{1} \mathrm{H}_{-} \mathrm{NMR} \_400 \mathrm{MHz}\left(\mathrm{CDCl}_{3}: 7.26 \mathrm{ppm}\right)\right]$

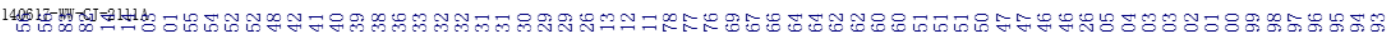
obo<smiles>O=CC[C@H]1C(c2ccccc2)=CCCC1c1ccnc2cc(Cl)ccc12</smiles>

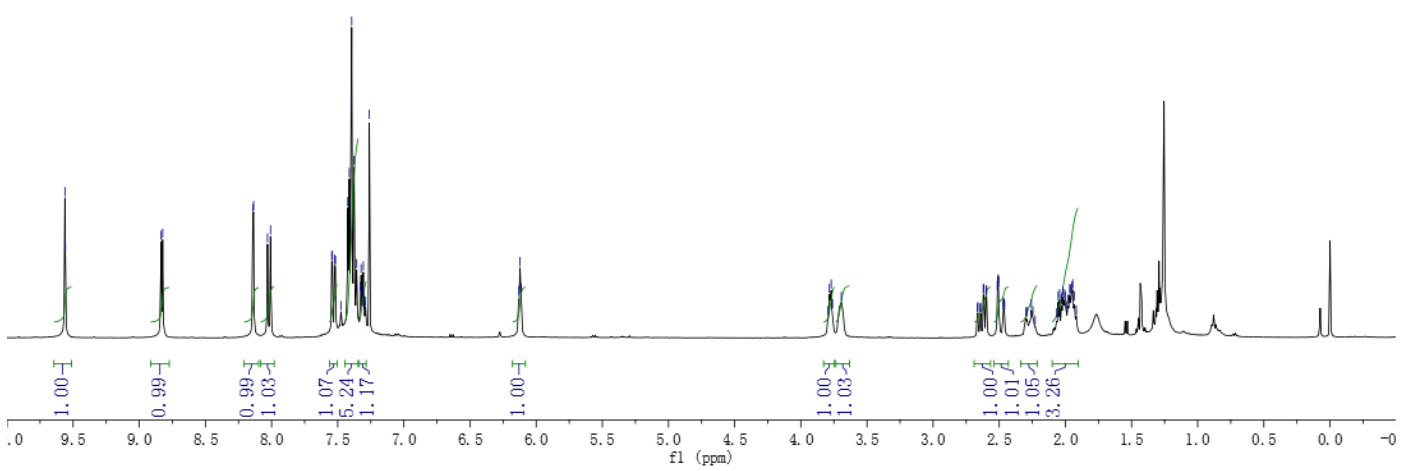

${ }^{13} \mathrm{C} \_\mathrm{NMR} \_100 \mathrm{MHz}\left(\mathrm{CDCl}_{3}: 77.00 \mathrm{ppm}\right)$

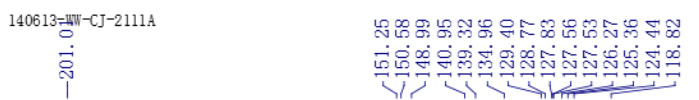

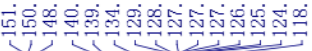<smiles>O=CC[C@H]1C(c2ccccc2)=CCCC1c1ccnc2cc(Cl)ccc12</smiles>

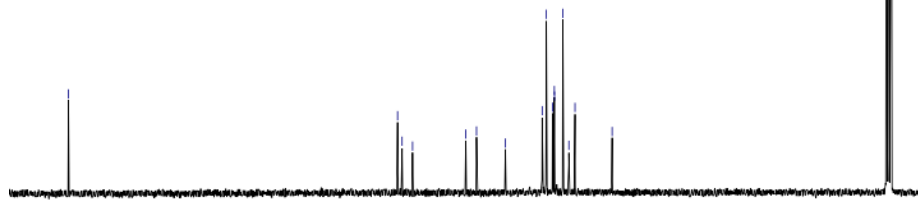

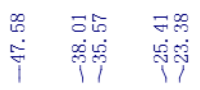

\begin{tabular}{llllllllllll}
\hline 10 & 200 & 190 & 180 & 170 & 160 & 150 & 140 & 130 & 120 & 110 & 100 \\
$\mathrm{fl}$ & $1 \mathrm{ppm})$
\end{tabular} 
2-((2R,3R)-3-(7-Chloroquinolin-4-yl)-2,3,4,5-tetrahydro-[1,1'-biphenyl]-2yl)acetaldehyde (3c') [ $\left.{ }^{1} \mathrm{H}_{-} \mathrm{NMR} \_400 \mathrm{MHz}\left(\mathrm{CDCl}_{3}: 7.26 \mathrm{ppm}\right)\right]$
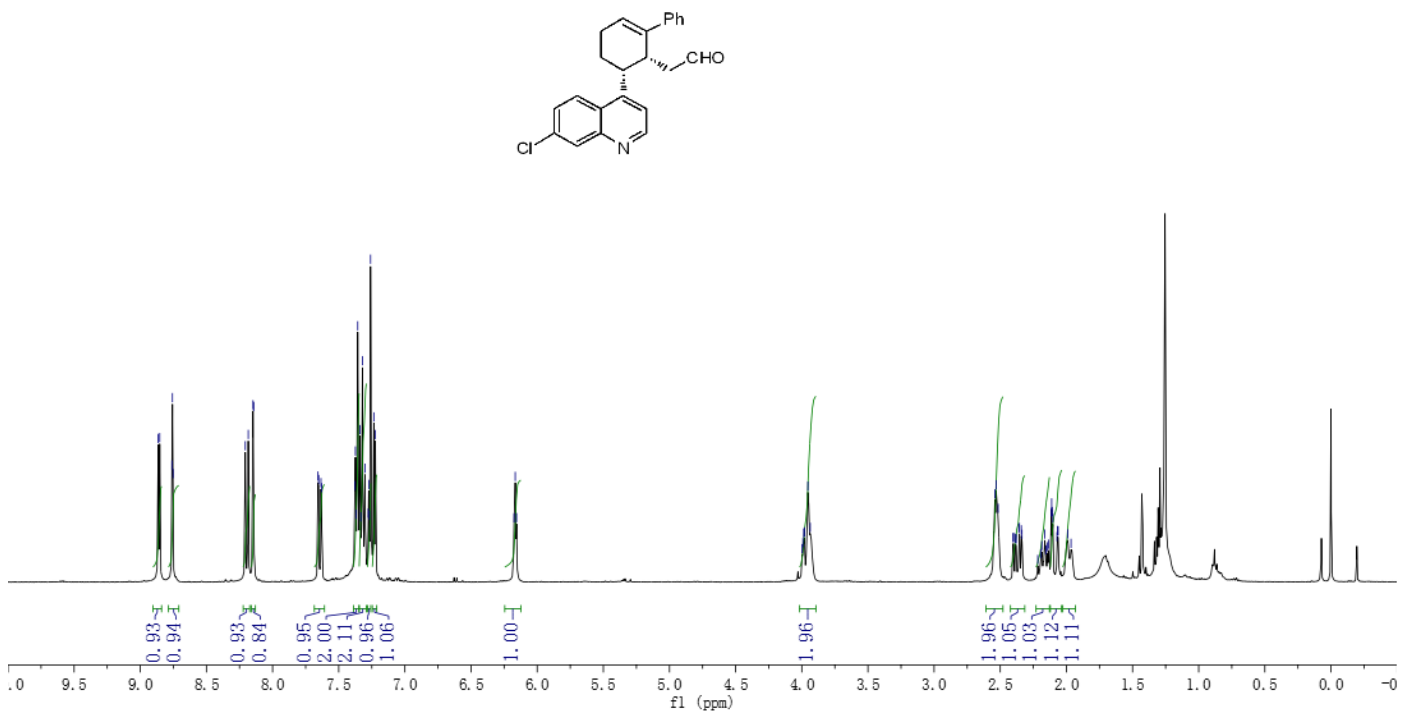

${ }^{13} \mathrm{C} \_N M R \_100 \mathrm{MHz}\left(\mathrm{CDCl}_{3}: 77.00 \mathrm{ppm}\right)$

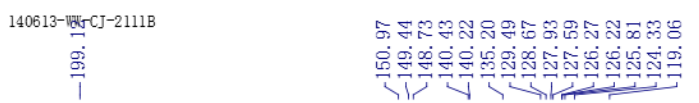

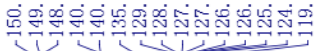
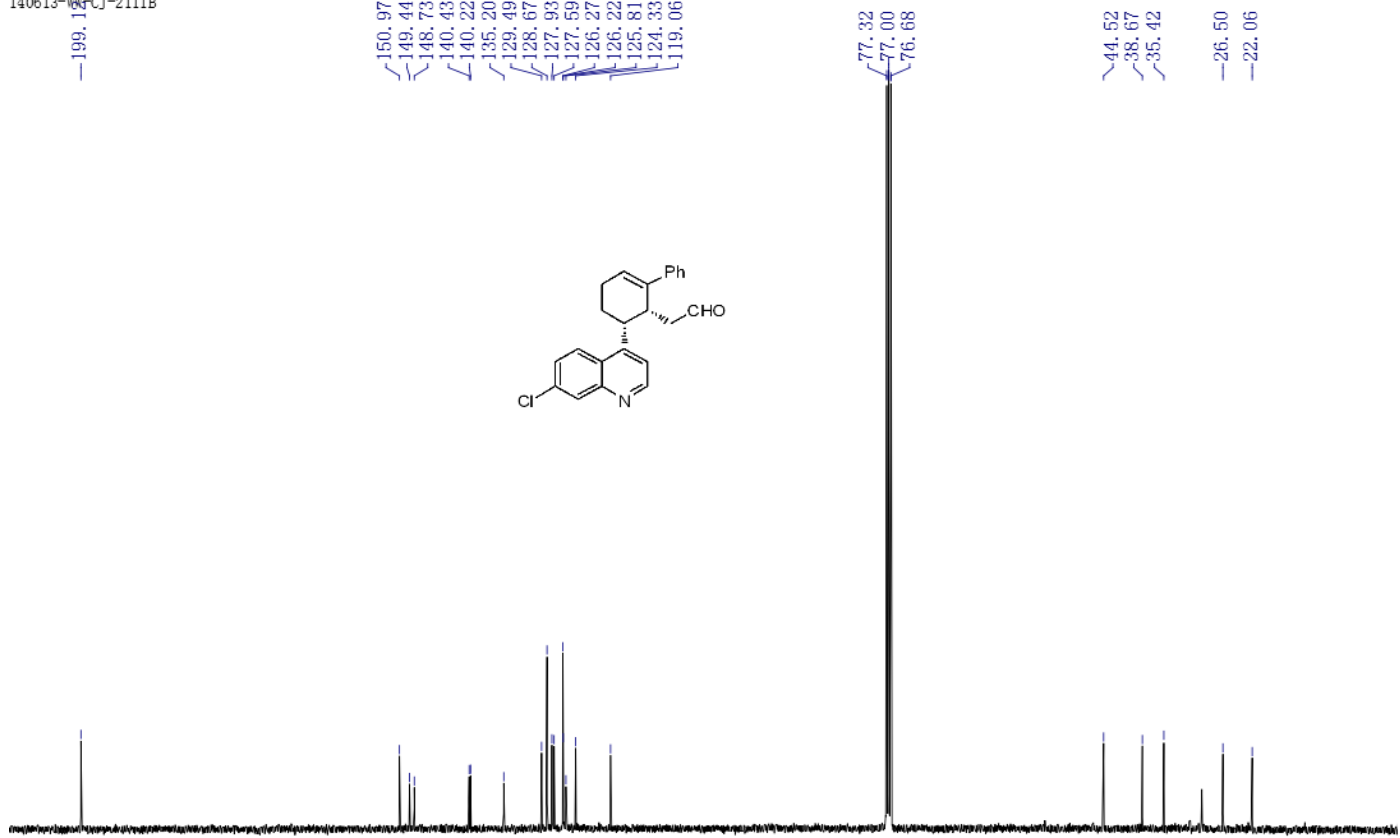

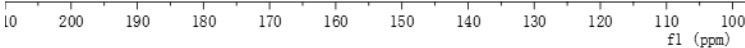


2-((2R,3S)-3-(7-(Trifluoromethyl)quinolin-4-yl)-2,3,4,5-tetrahydro-[1,1'-biphenyl]-2yl)acetaldehyde (3d) $\left[{ }^{1} \mathrm{H} \_\mathrm{NMR} \_400 \mathrm{MHz}\left(\mathrm{CDCl}_{3}: 7.26 \mathrm{ppm}\right)\right]$
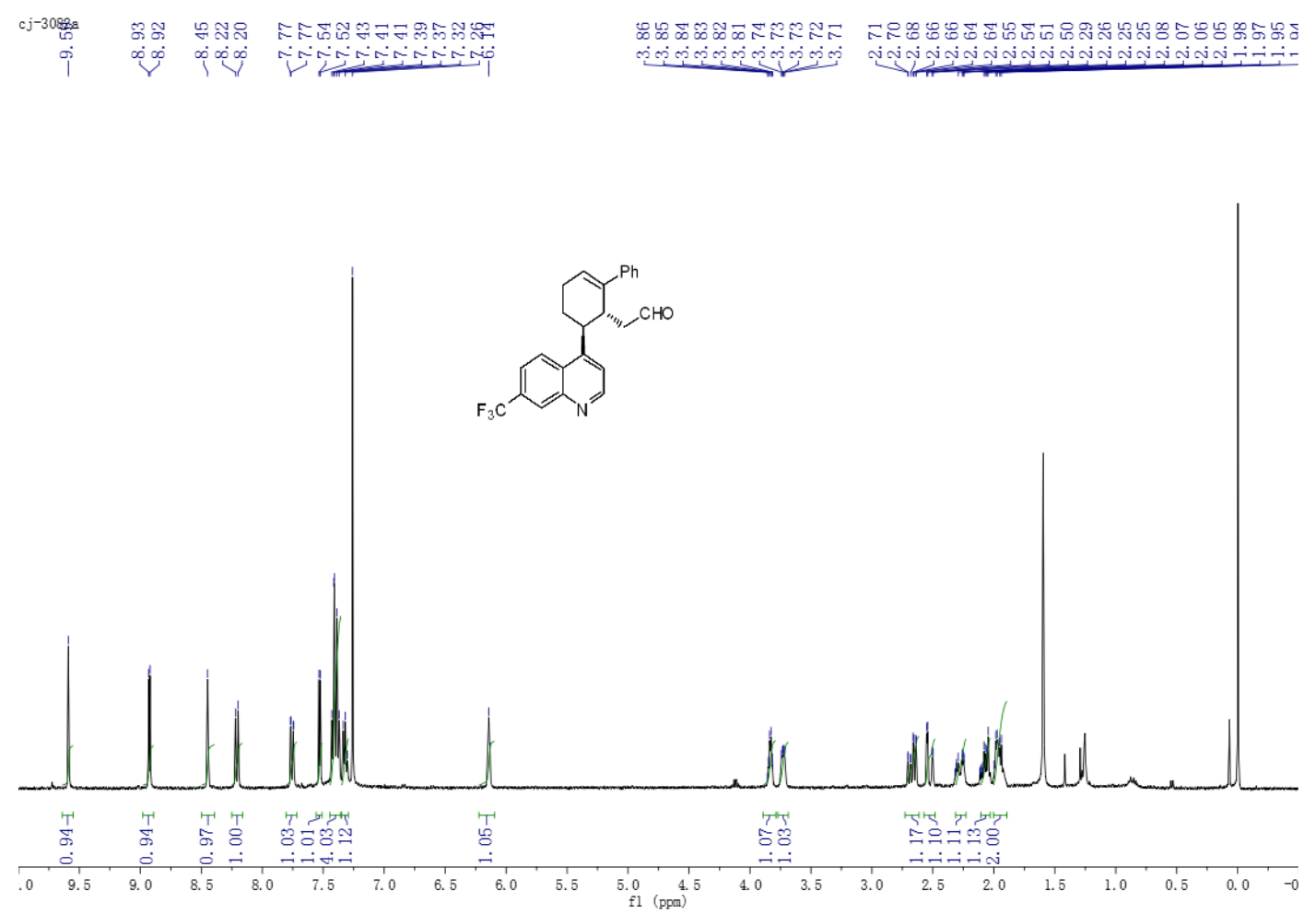

${ }^{13} \mathrm{C} \_N M R \_100 \mathrm{MHz}\left(\mathrm{CDCl}_{3}: 77.00 \mathrm{ppm}\right)$

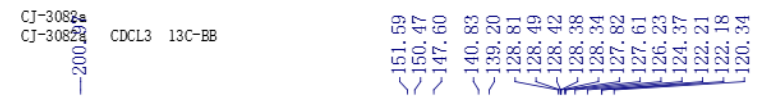

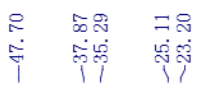

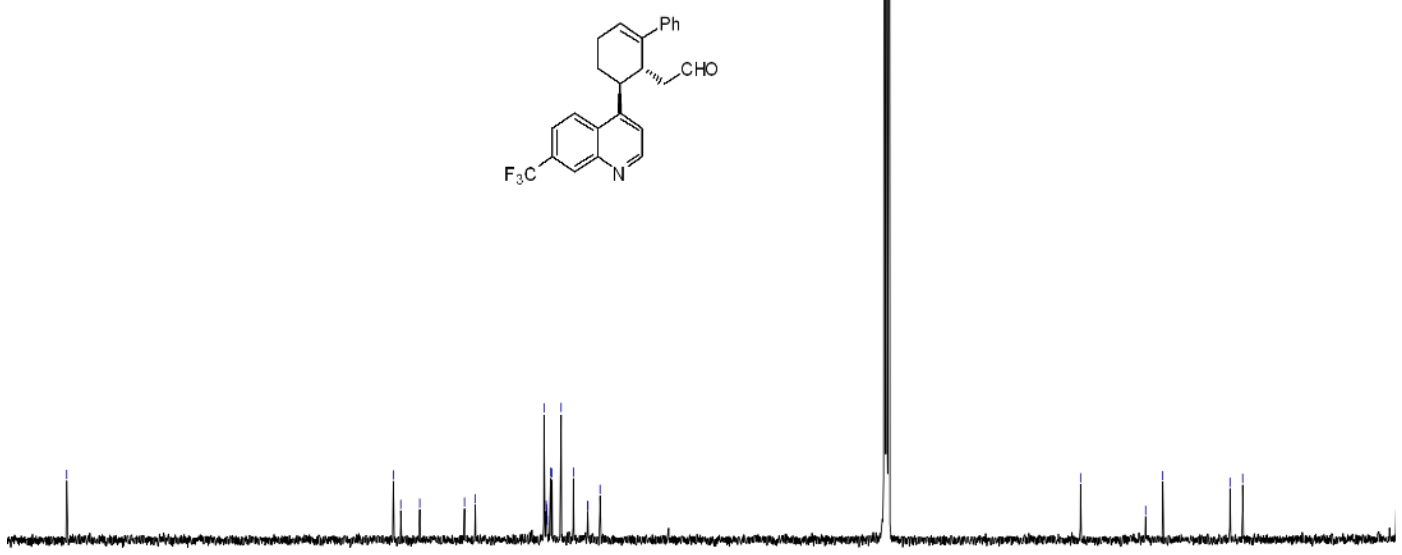

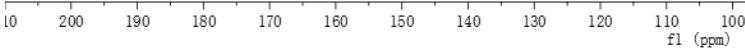


2-((2R,3R)-3-(7-(Trifluoromethyl)quinolin-4-yl)-2,3,4,5-tetrahydro-[1,1'-biphenyl]-2yl)acetaldehyde (3d') [ $\left.{ }^{1} \mathrm{H}_{-} \mathrm{NMR} \_400 \mathrm{MHz}\left(\mathrm{CDCl}_{3}: 7.26 \mathrm{ppm}\right)\right]$

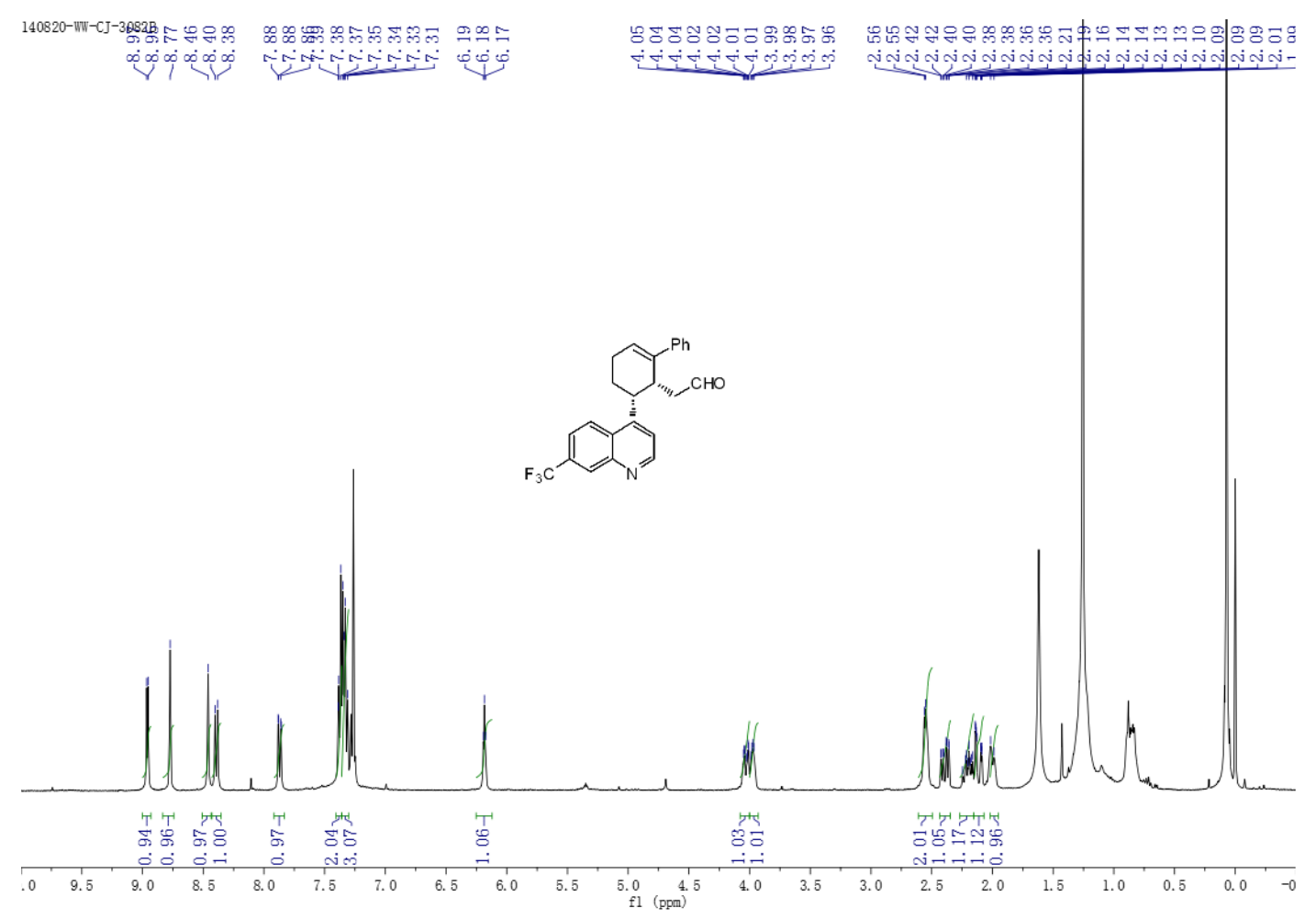

${ }^{13} \mathrm{C} \_N M R \_100 \mathrm{MHz}\left(\mathrm{CDCl}_{3}: 77.00 \mathrm{ppm}\right)$

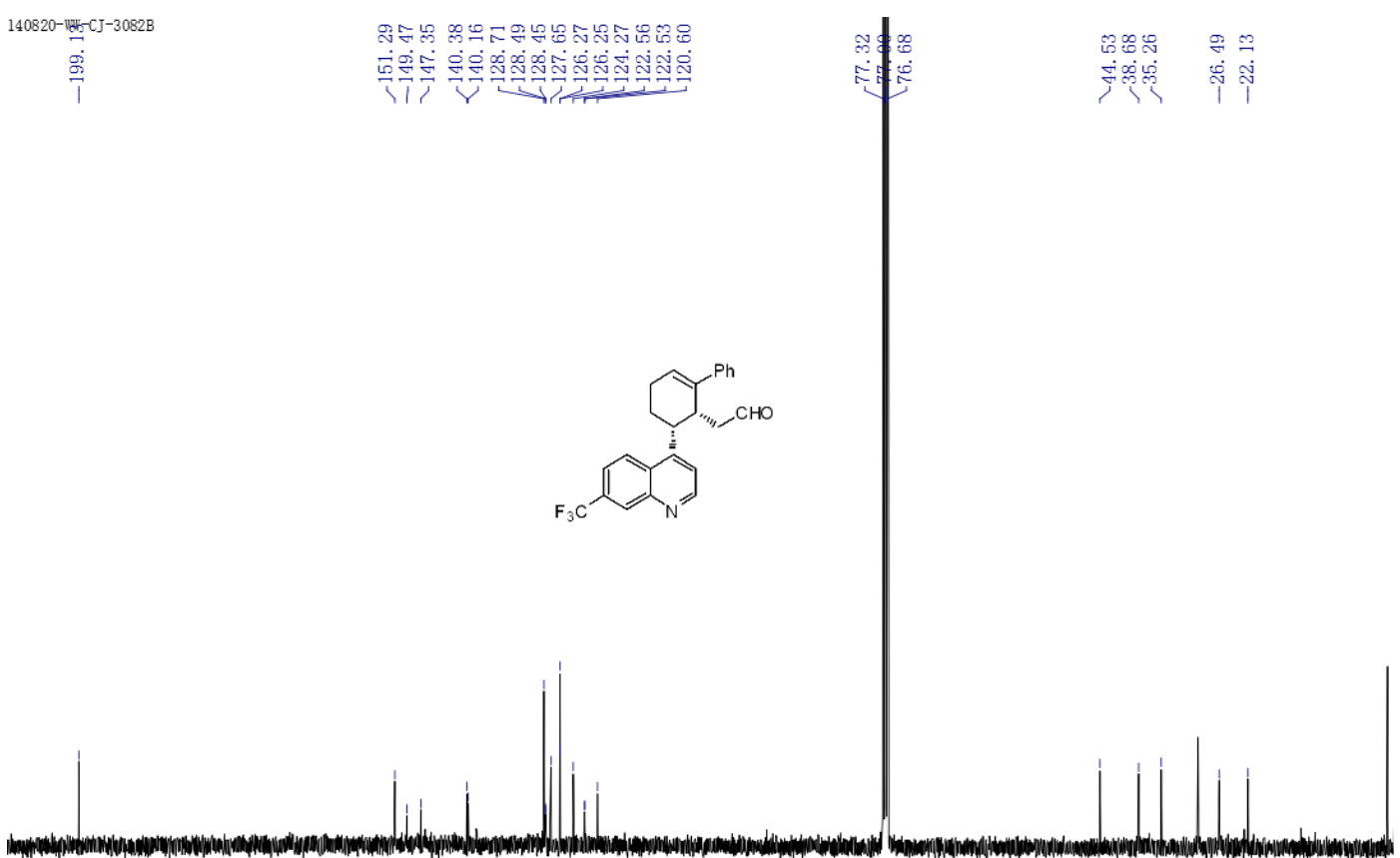

$\begin{array}{llllllllllll}10 & 200 & 190 & 180 & 170 & 160 & 150 & 140 & 130 & 120 & 110 & 100 \\ \mathrm{fl} & (\mathrm{ppm})\end{array}$ 
2-((2R,3S)-3-(6-Fluoroquinolin-4-yl)-2,3,4,5-tetrahydro-[1,1'-biphenyl]-2yl)acetaldehyde (3e) $\left[{ }^{1} \mathrm{H}_{-} \mathrm{NMR} \_400 \mathrm{MHz}\left(\mathrm{CDCl}_{3}: 7.26 \mathrm{ppm}\right)\right]$

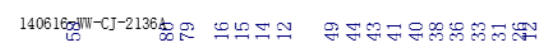

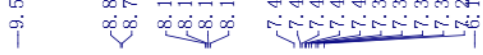

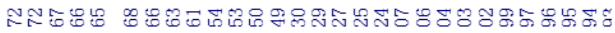

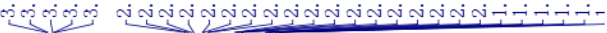<smiles>O=CC[C@H]1C(c2ccccc2)=CCCC1c1ccnc2ccc(F)cc12</smiles>

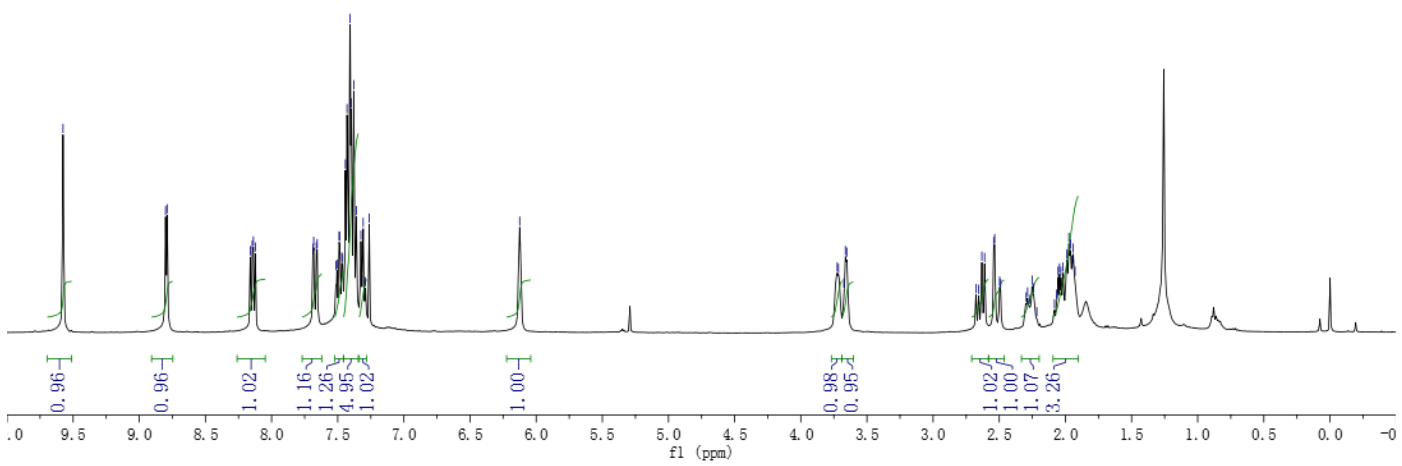

${ }^{13} \mathrm{C} \_N M R \_100 \mathrm{MHz}\left(\mathrm{CDCl}_{3}: 77.00 \mathrm{ppm}\right)$
$140616=-1 \mathrm{WW}-\mathrm{CJ}-2136 \mathrm{~A}$

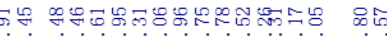
灾

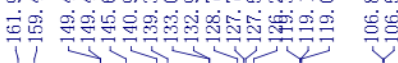

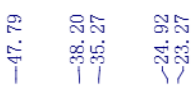
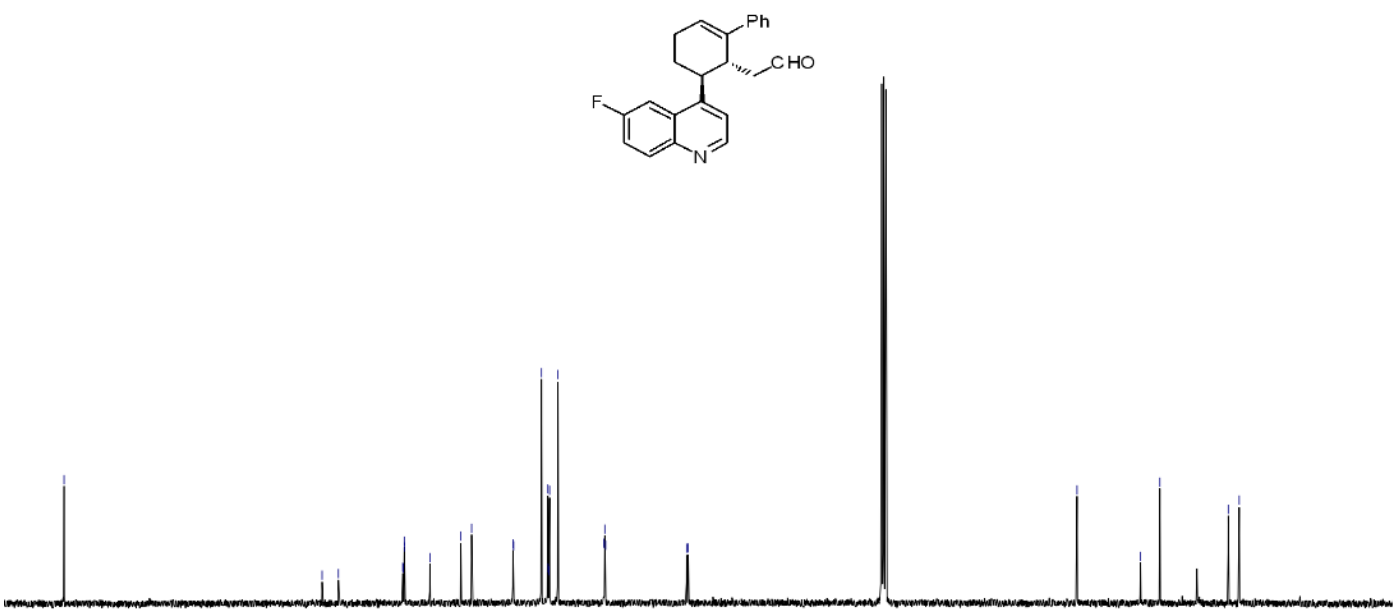

$\begin{array}{lllllllllll}10 & 200 & 190 & 180 & 170 & 160 & 150 & 140 & 130 & 120 & 110 \\ \mathrm{f} 1 \text { (ppm) }\end{array}$ 
2-((2R,3R)-3-(6-Fluoroquinolin-4-yl)-2,3,4,5-tetrahydro-[1,1'-biphenyl]-2yl)acetaldehyde (3e') [ $\left.{ }^{1} \mathrm{H}_{-} \mathrm{NMR} \_400 \mathrm{MHz}\left(\mathrm{CDCl}_{3}: 7.26 \mathrm{ppm}\right)\right]$
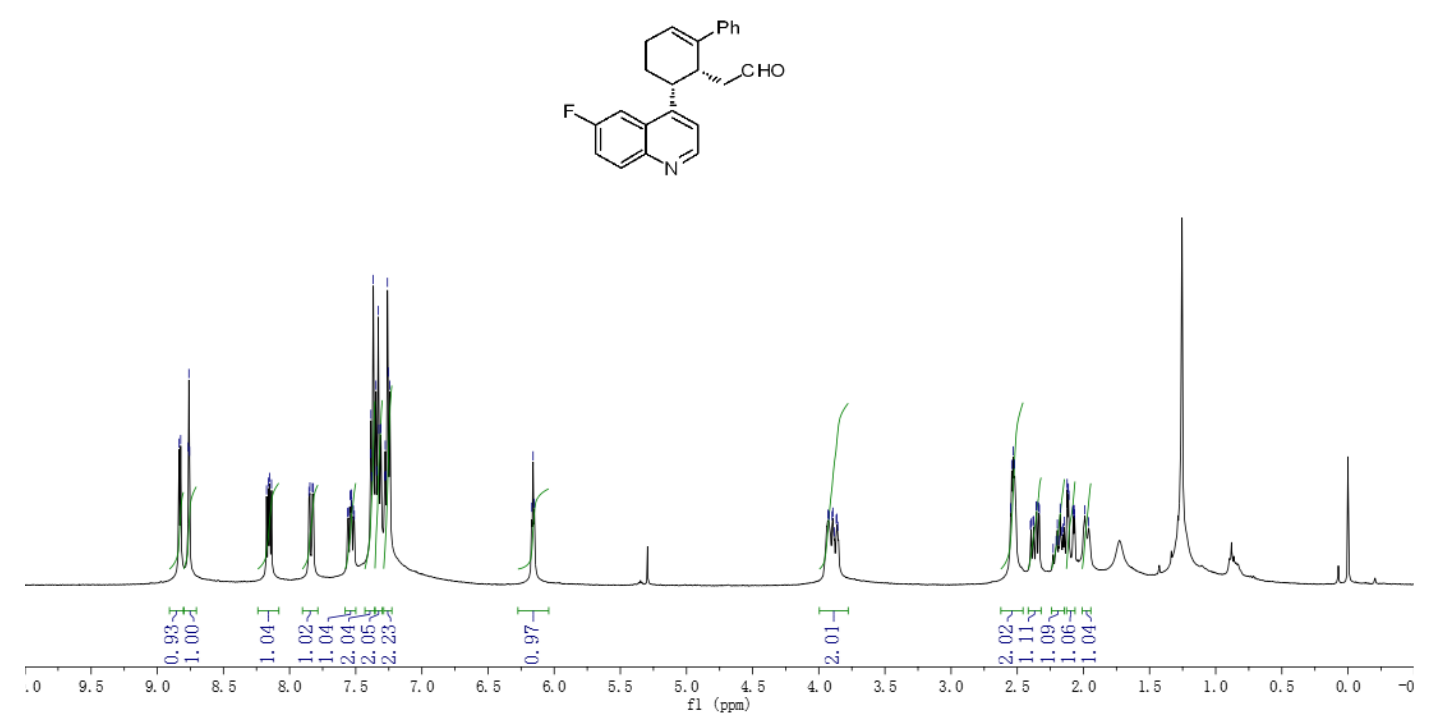

${ }^{13} \mathrm{C} \_$NMR_100 MHz_(CDCl $\left.3: 77.00 \mathrm{ppm}\right)$

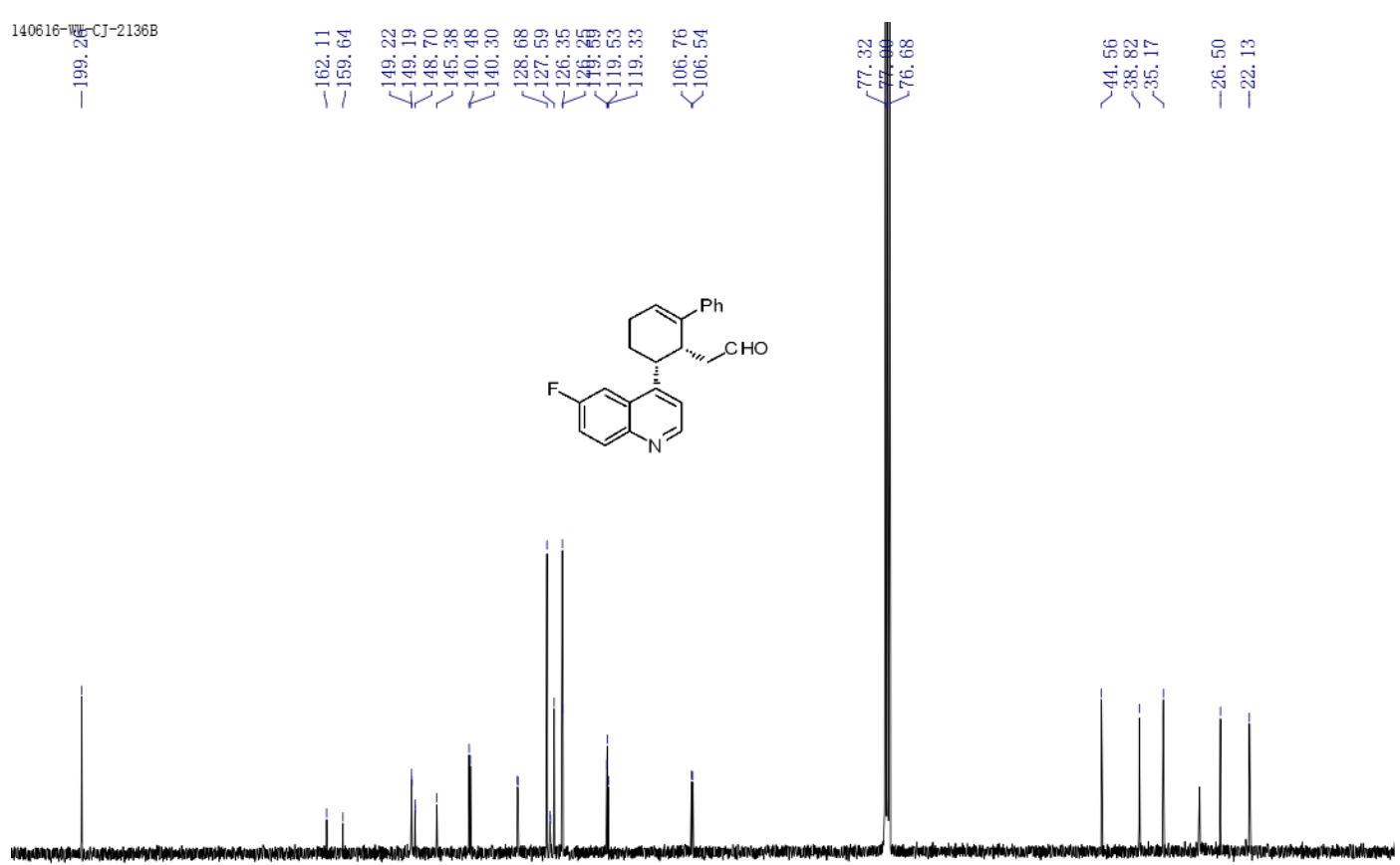

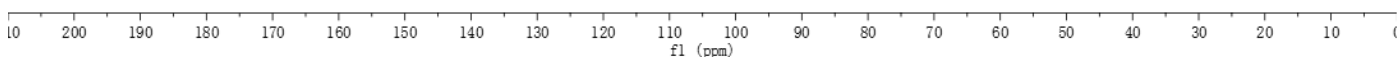


2-((2R,3S)-3-(6-methylquinolin-4-yl)-2,3,4,5-tetrahydro-[1,1'-biphenyl]-2yl)acetaldehyde (3f) $\left[{ }^{1} \mathrm{H} \_\mathrm{NMR} \_400 \mathrm{MHz}\left(\mathrm{CDCl}_{3}: 7.26 \mathrm{ppm}\right)\right]$

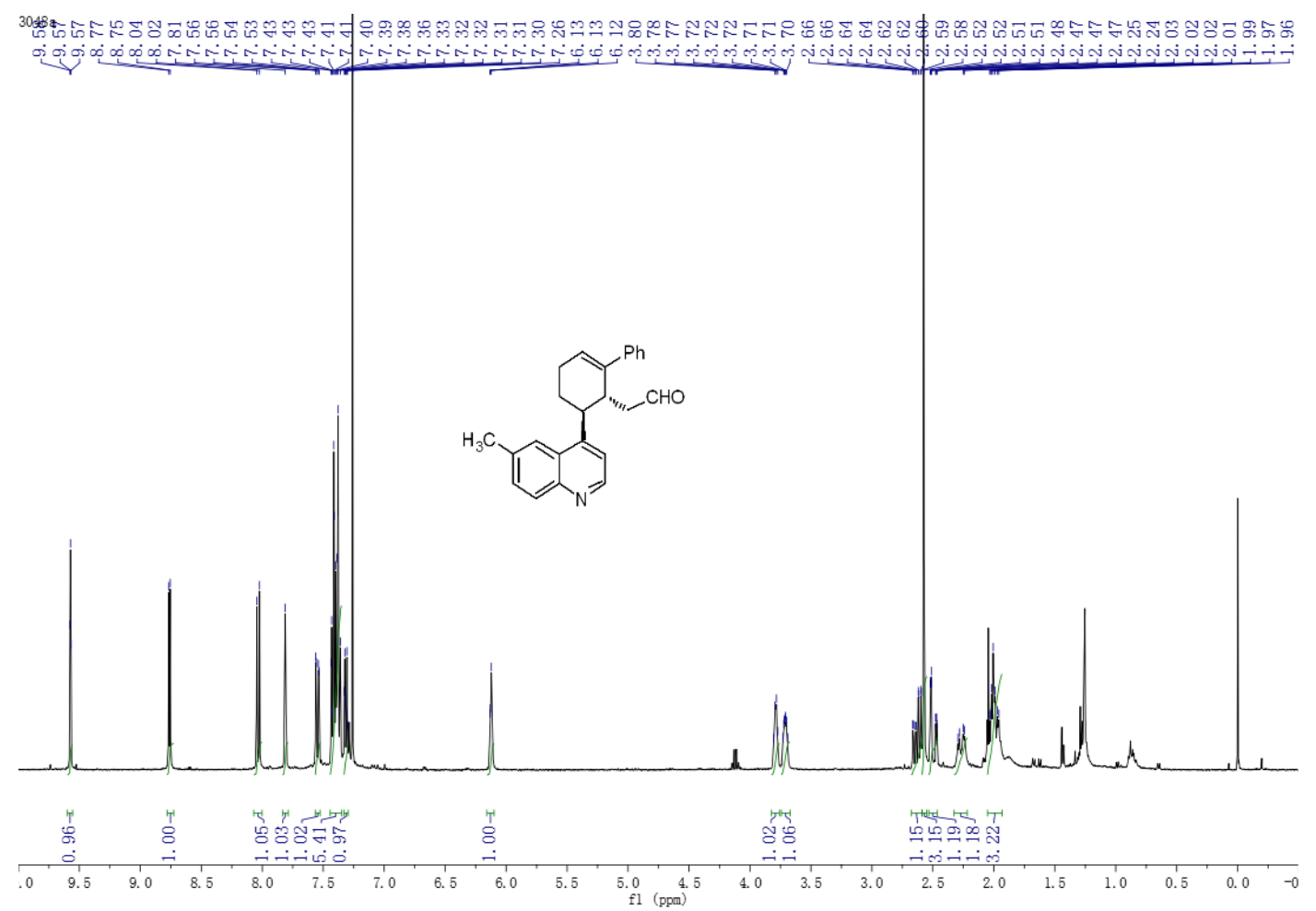

${ }^{13} \mathrm{C} \_N M R \_100 \mathrm{MHz}\left(\mathrm{CDCl}_{3}: 77.00 \mathrm{ppm}\right)$

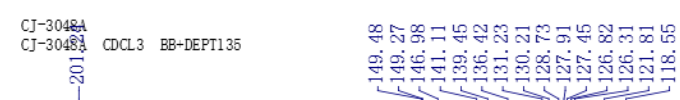
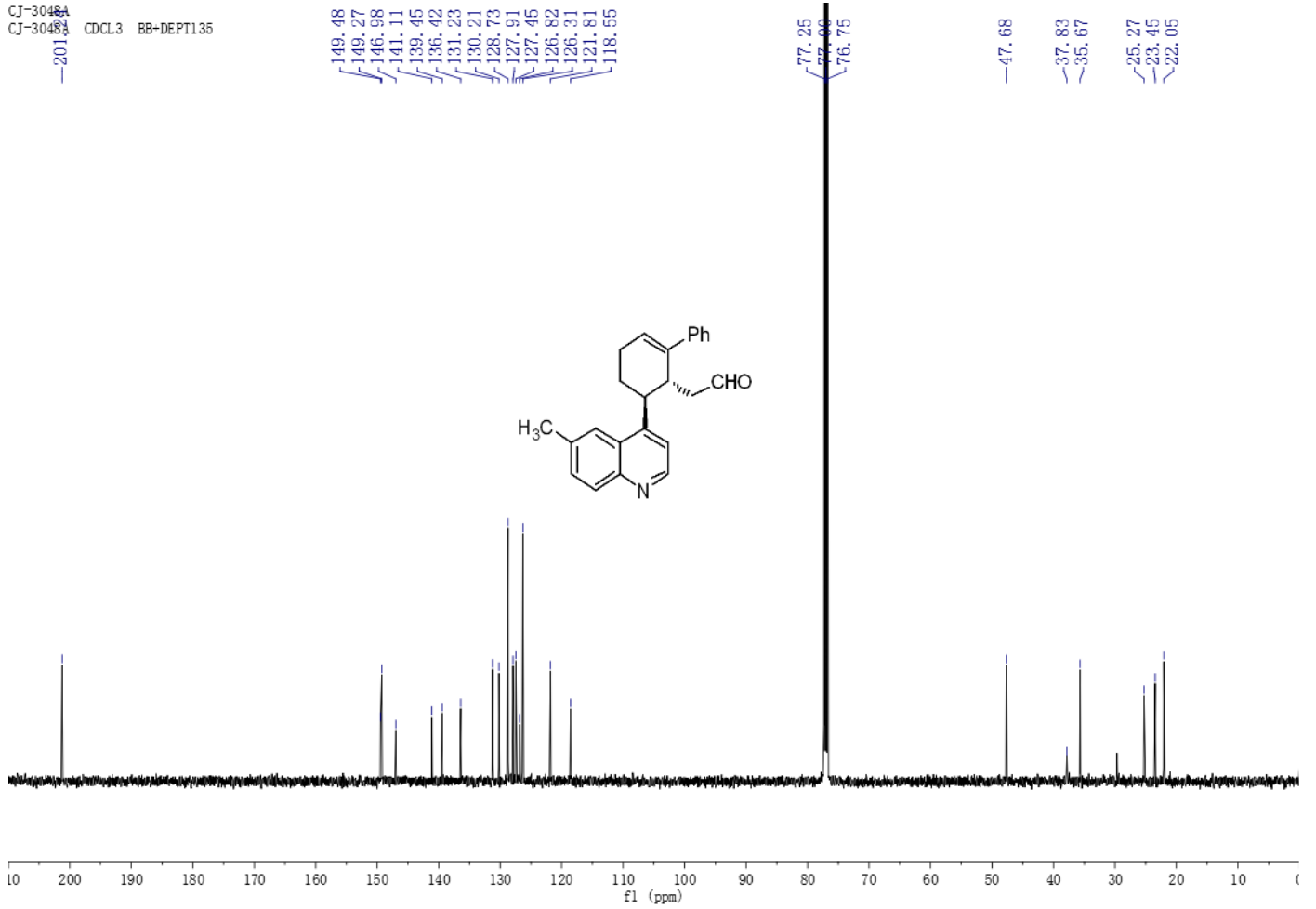
2-((2R,3R)-3-(6-methylquinolin-4-yl)-2,3,4,5-tetrahydro-[1,1'-biphenyl]-2yl)acetaldehyde (3f') [ $\left.{ }^{1} \mathrm{H}_{-} \mathrm{NMR} \_400 \mathrm{MHz}\left(\mathrm{CDCl}_{3}: 7.26 \mathrm{ppm}\right)\right]$

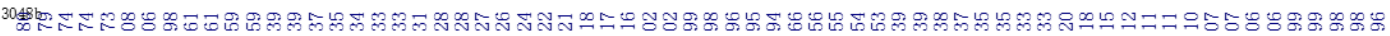

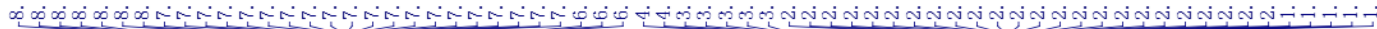

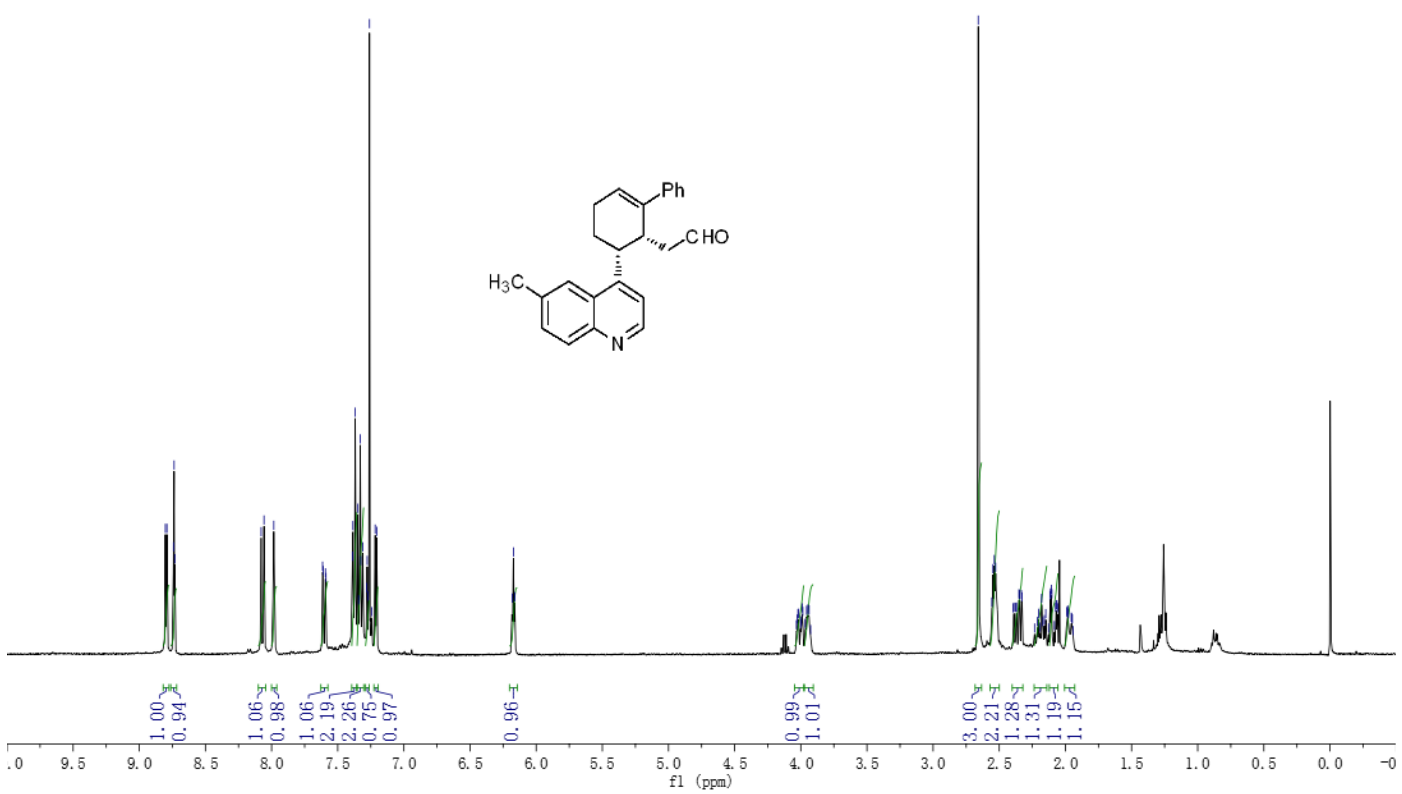

${ }^{13} \mathrm{C} \_\mathrm{NMR} \_100 \mathrm{MHz}\left(\mathrm{CDCl}_{3}: 77.00 \mathrm{ppm}\right)$

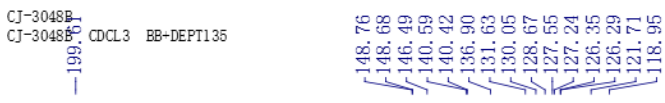
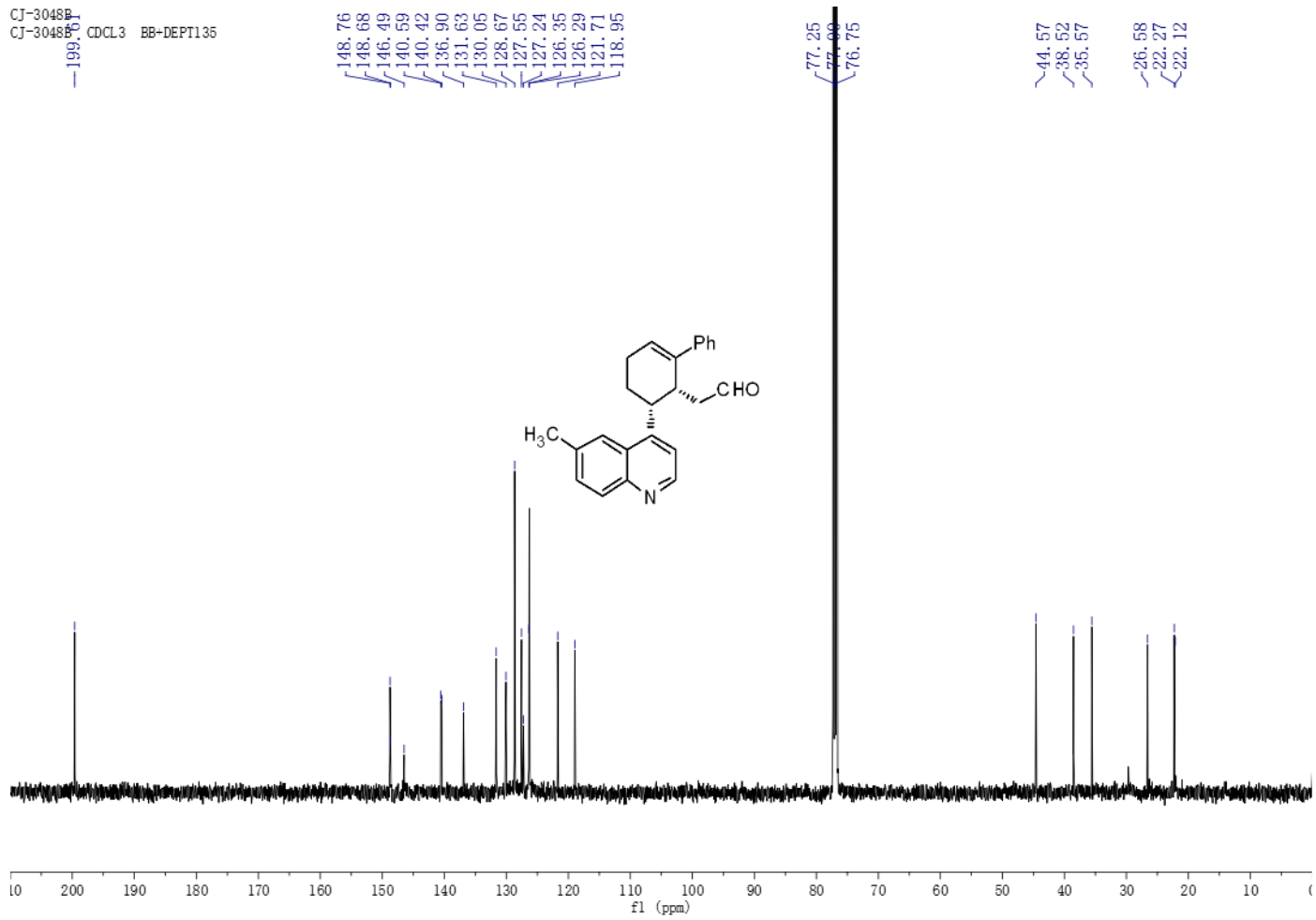
2-((2R,3S)-3-(2-Methylquinolin-4-yl)-2,3,4,5-tetrahydro-[1,1'-biphenyl]-2yl)acetaldehyde (3g) [ $\left.{ }^{1} \mathrm{H}_{-} \mathrm{NMR} \_400 \mathrm{MHz}\left(\mathrm{CDCl}_{3}: 7.26 \mathrm{ppm}\right)\right]$

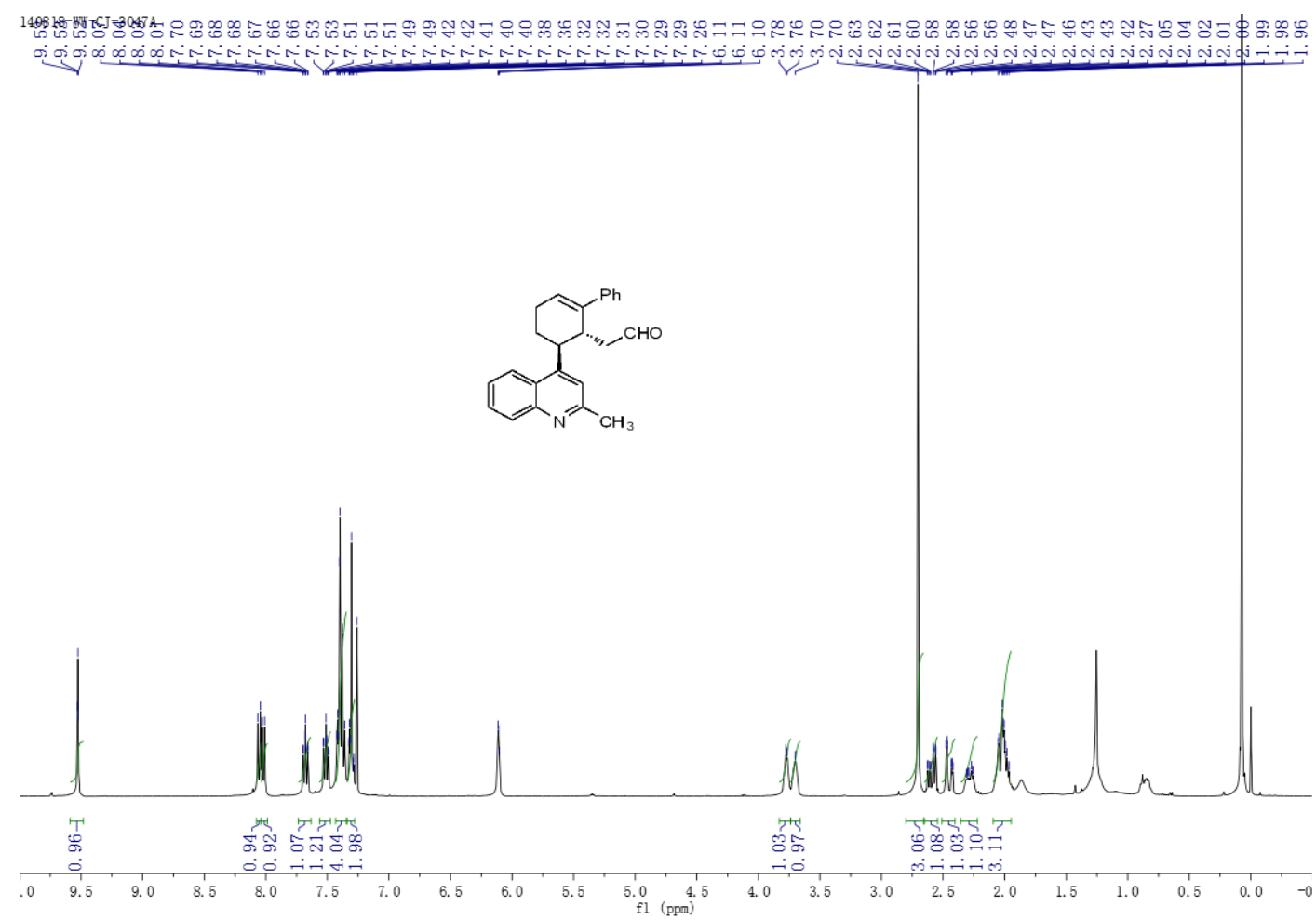

${ }^{13} \mathrm{C} \_N M R \_100 \mathrm{MHz}\left(\mathrm{CDCl}_{3}: 77.00 \mathrm{ppm}\right)$
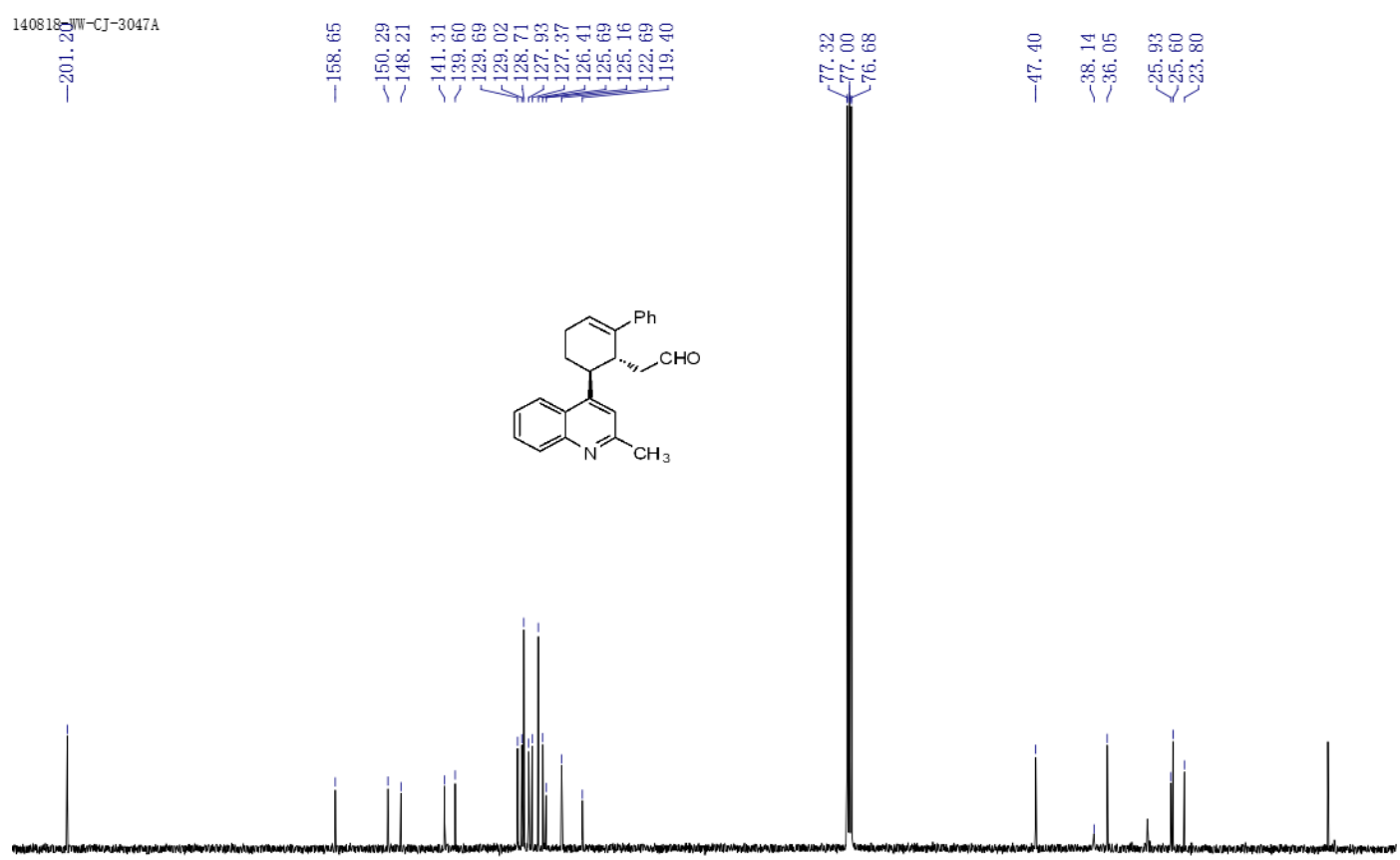

$\begin{array}{llllllllllll}10 & 200 & 190 & 180 & 170 & 160 & 150 & 140 & 130 & 120 & 110 & 100 \\ \mathrm{fl}(\mathrm{ppm}) & \end{array}$ 
2-((2R,3R)-3-(2-Methylquinolin-4-yl)-2,3,4,5-tetrahydro-[1,1'-biphenyl]-2yl)acetaldehyde (3g') [ $\left.{ }^{1} \mathrm{H}_{-} \mathrm{NMR} \_400 \mathrm{MHz}\left(\mathrm{CDCl}_{3}: 7.26 \mathrm{ppm}\right)\right]$

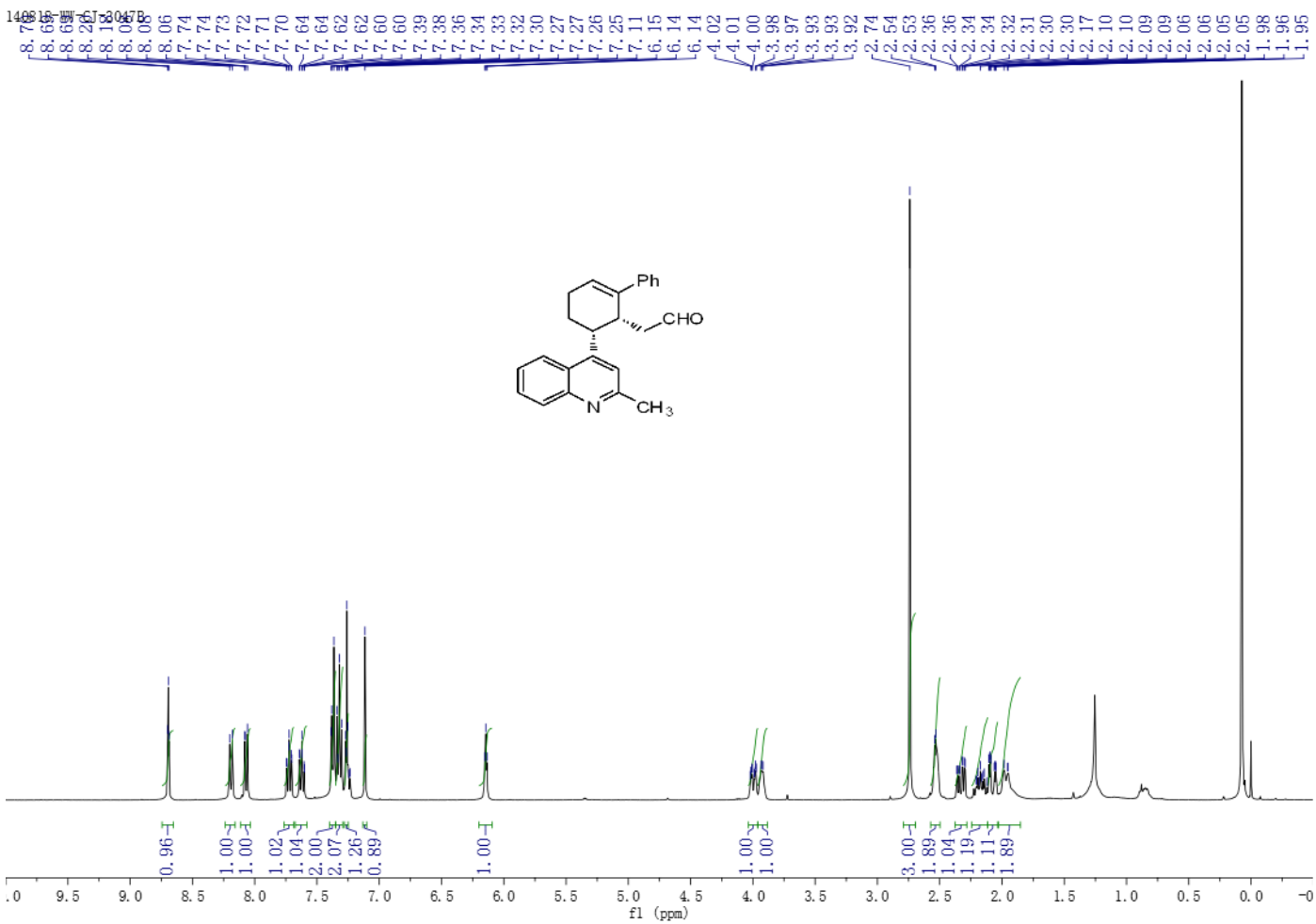

${ }^{13} \mathrm{C} \_N M R \_100 \mathrm{MHz}\left(\mathrm{CDCl}_{3}: 77.00 \mathrm{ppm}\right)$

\begin{tabular}{|c|c|c|c|c|}
\hline 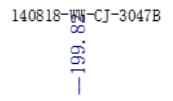 & $\begin{array}{l}\vec{b} \\
\infty \\
\stackrel{\infty}{0} \\
\stackrel{1}{T}\end{array}$ & 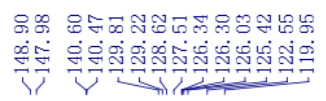 & 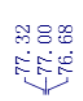 & 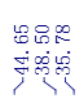 \\
\hline
\end{tabular}
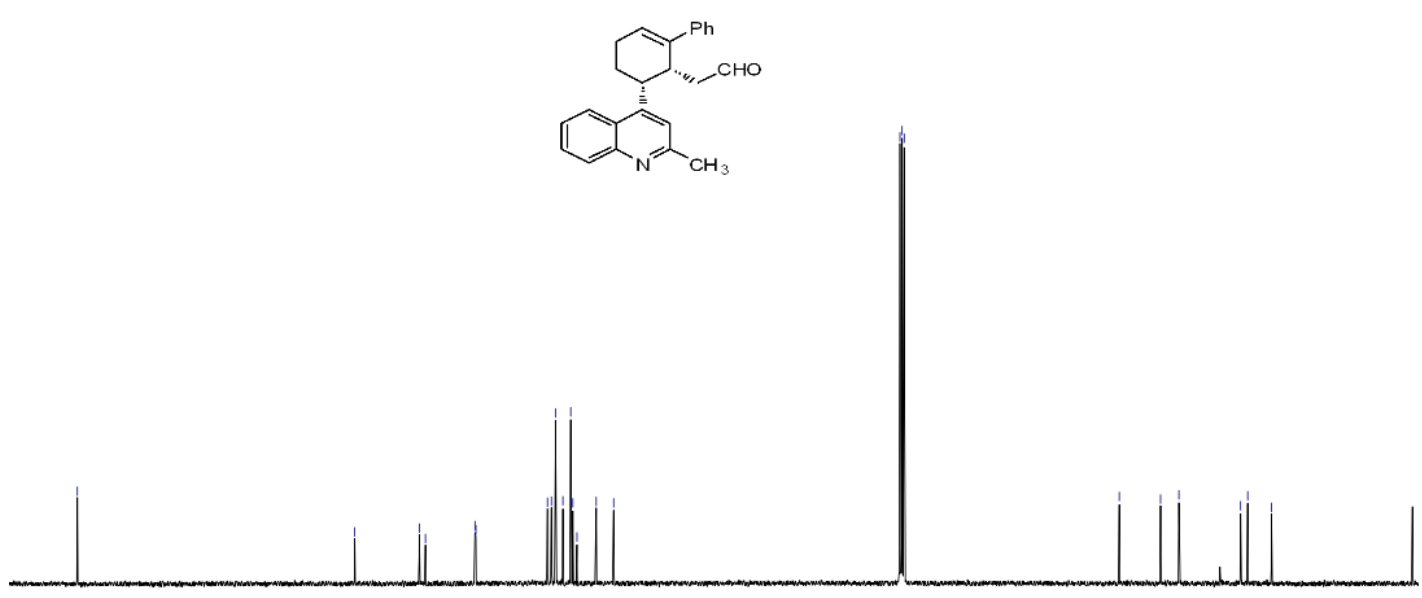

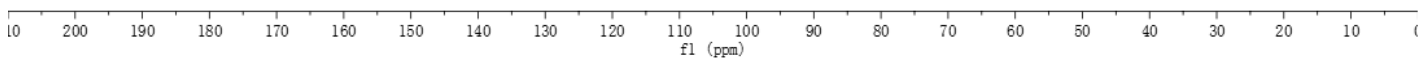


2-((1R,6S)-2-Methyl-6-(quinolin-4-yl)cyclohex-2-en-1-yl)acetaldehyde

(3h) $\left[{ }^{1} \mathrm{H} \_\mathrm{NMR} \_400 \mathrm{MHz}\left(\mathrm{CDCl}_{3}: 7.26 \mathrm{ppm}\right)\right]$

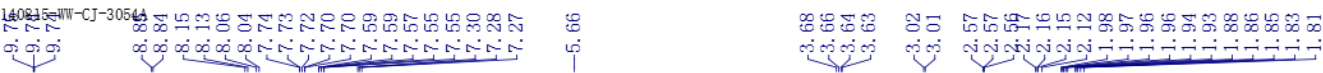

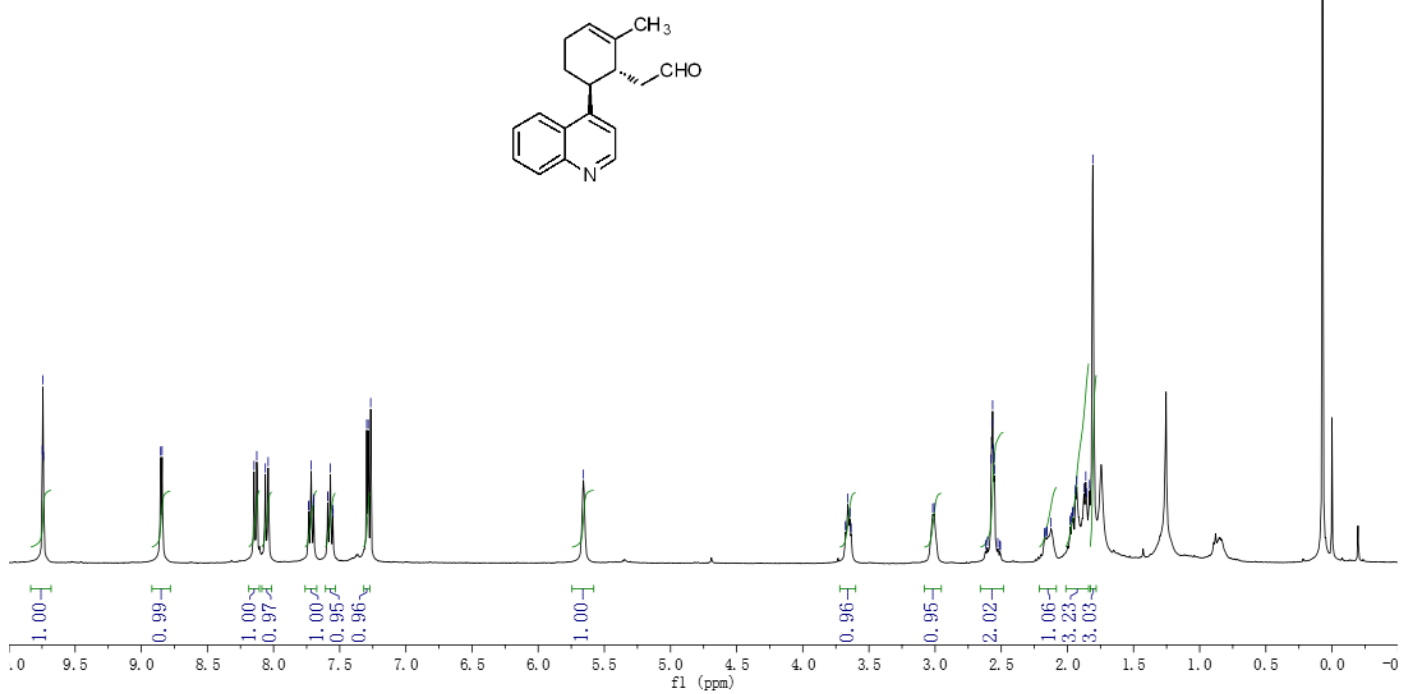

${ }^{13} \mathrm{C} \_\mathrm{NMR} \_100 \mathrm{MHz}\left(\mathrm{CDCl}_{3}: 77.00 \mathrm{ppm}\right)$

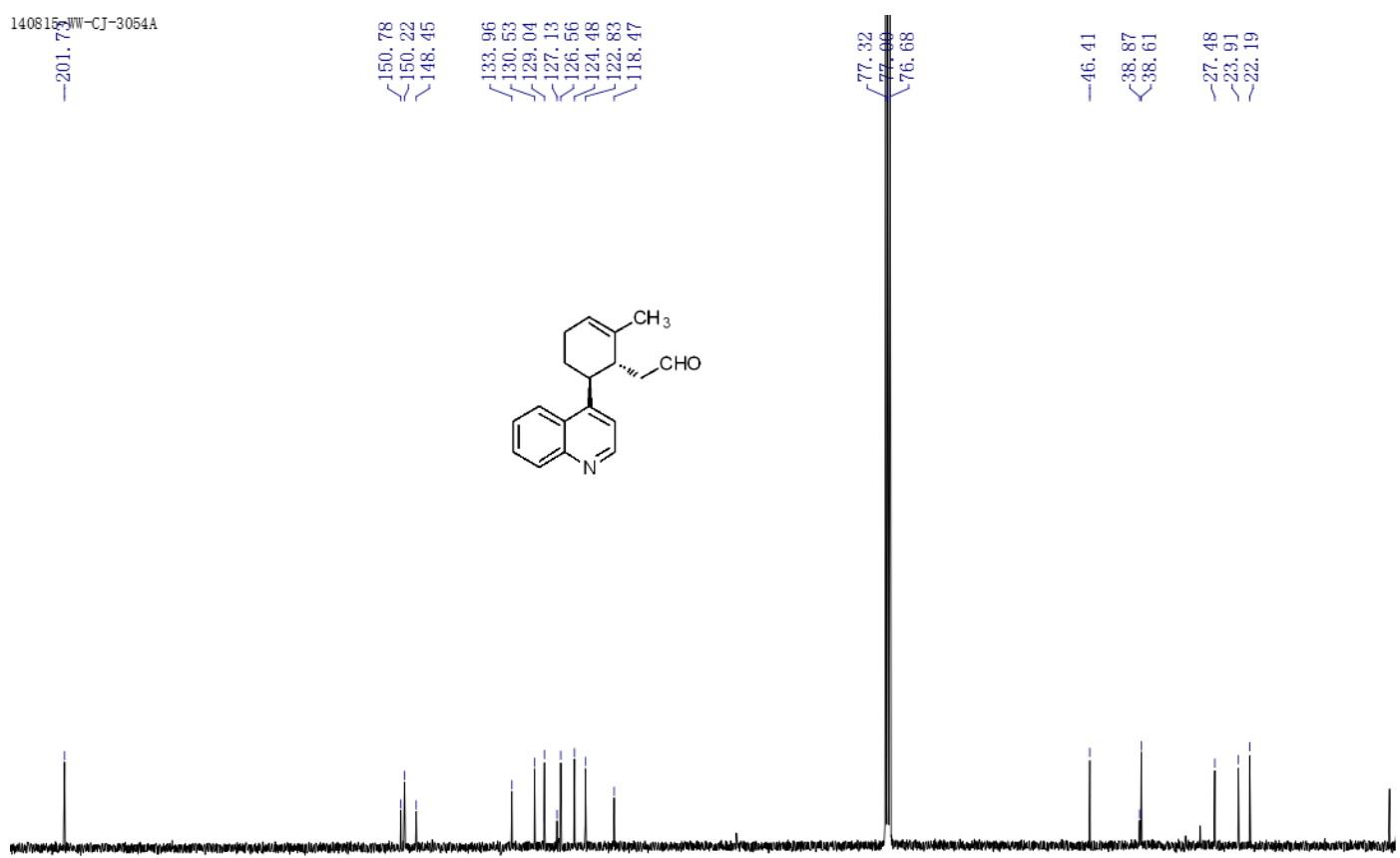

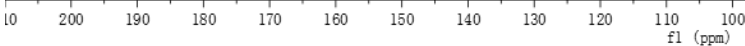


2-((1R,6R)-2-Methyl-6-(quinolin-4-yl)cyclohex-2-en-1-yl)acetaldehyde $\left[{ }^{1} \mathrm{H} \_\mathrm{NMR} \_400 \mathrm{MHz}\left(\mathrm{CDCl}_{3}: 7.26 \mathrm{ppm}\right)\right]$
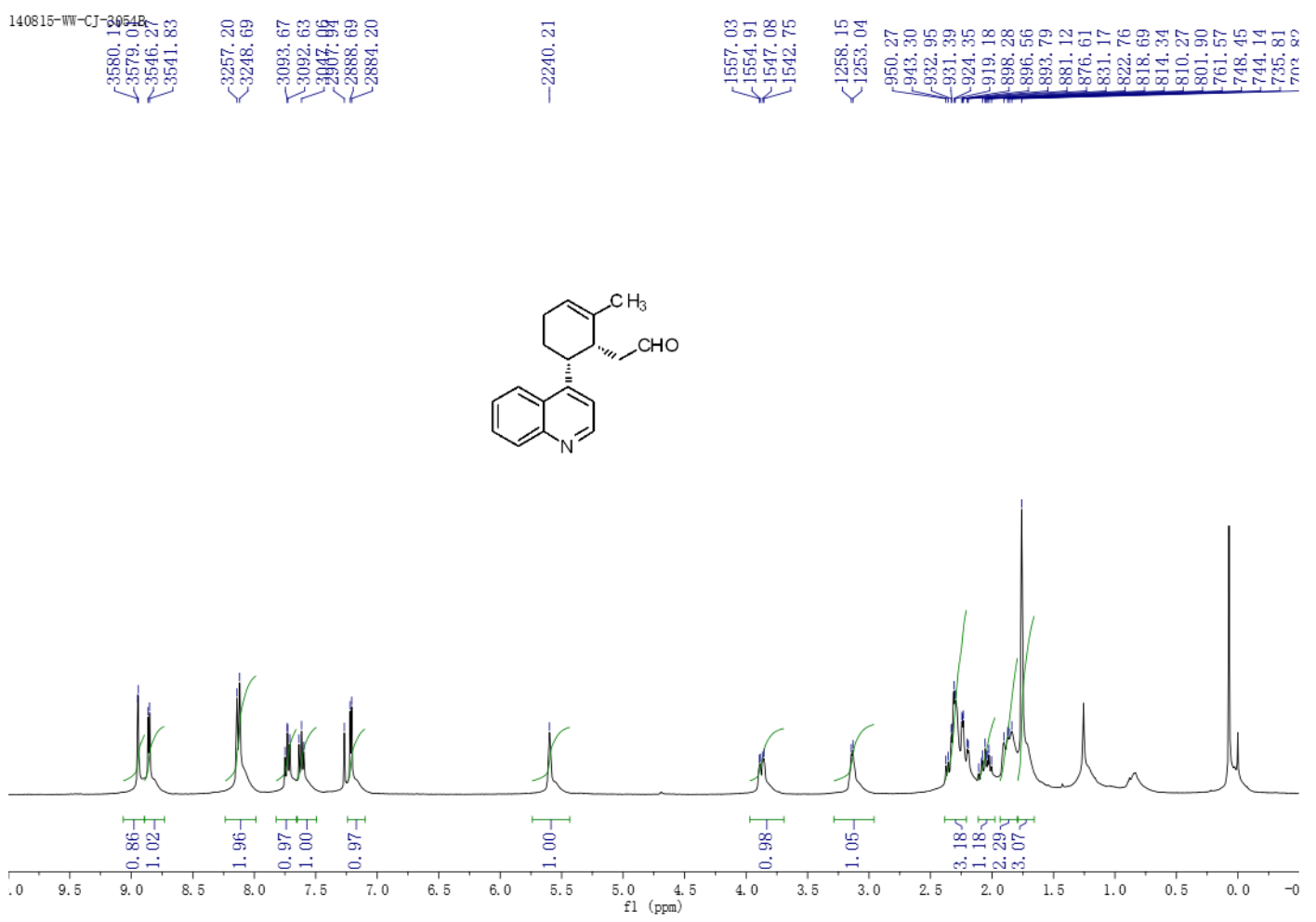

${ }^{13} \mathrm{C} \_\mathrm{NMR} \_100 \mathrm{MHz}\left(\mathrm{CDCl}_{3}: 77.00 \mathrm{ppm}\right)$

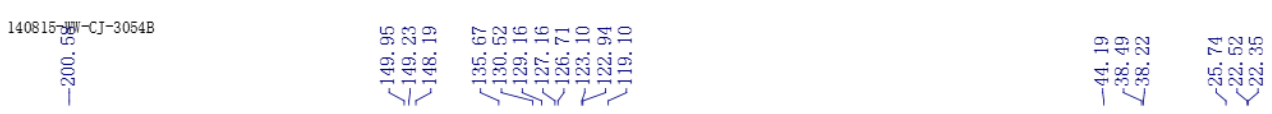
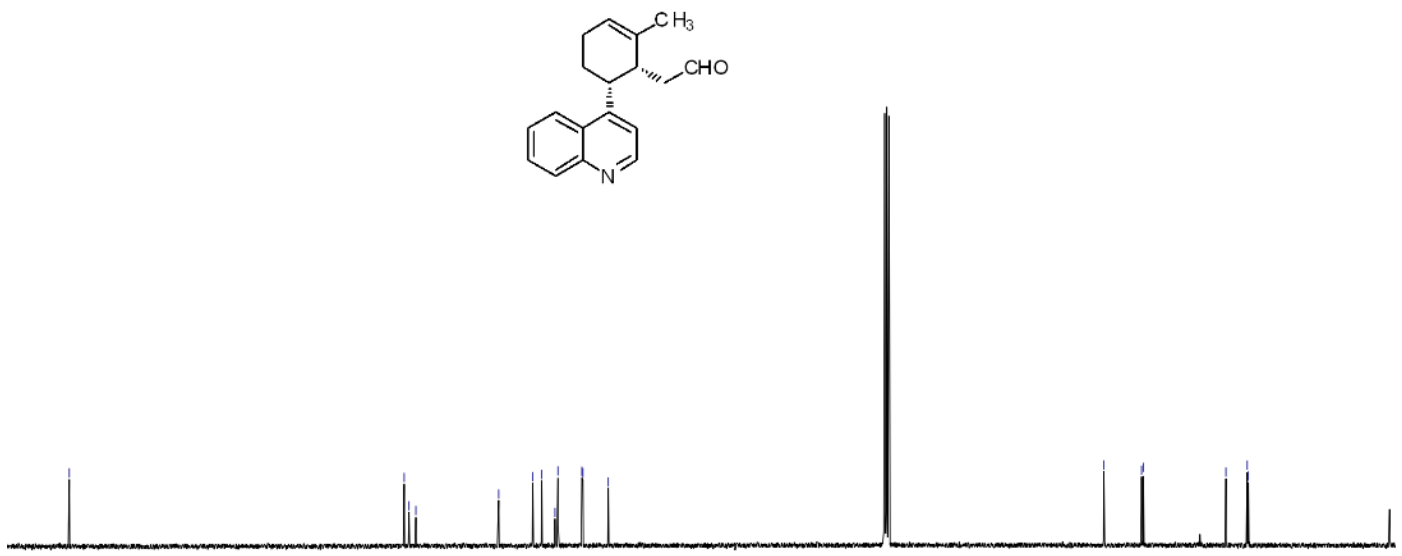

\begin{tabular}{lllllllllll}
\hline 10 & 200 & 190 & 180 & 170 & 160 & 150 & 140 & 130 & 120 & 110 \\
$\mathrm{fl} 1(\mathrm{ppm})$ & 100
\end{tabular} 
2-((2R,3S)-5-Methyl-3-(quinolin-4-yl)-2,3,4,5-tetrahydro-[1,1'-biphenyl]-2yl)acetaldehyde (3i) $\left[{ }^{1} \mathrm{H}_{-} \mathrm{NMR} \_400 \mathrm{MHz}\left(\mathrm{CDCl}_{3}: 7.26 \mathrm{ppm}\right)\right]$

140815-

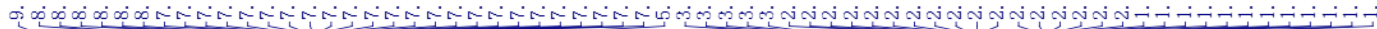<smiles>CC1C=C(P)C(CO)C(CO)C1c1ccnc2ccccc12</smiles>

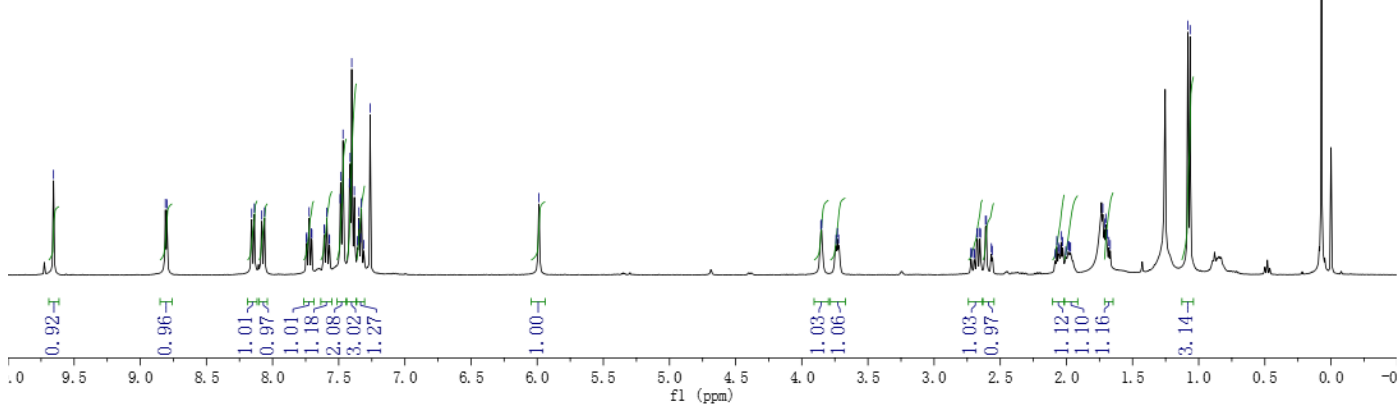

${ }^{13} \mathrm{C} \_\mathrm{NMR} \_100 \mathrm{MHz}\left(\mathrm{CDCl}_{3}: 77.00 \mathrm{ppm}\right)$

\begin{tabular}{|c|c|}
\hline $140815=$ =HIT $-\mathrm{CJ}-3055 \mathrm{~A}$ & \\
\hline ¿্ं & 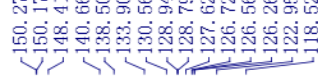 \\
\hline
\end{tabular}<smiles>CC1C=C(P)[C@H](CC=O)C(c2ccnc3ccccc23)C1</smiles>

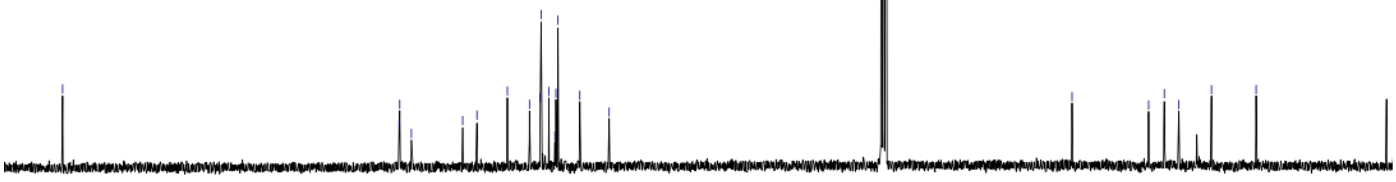

$\begin{array}{lllllllllll}10 & 200 & 190 & 180 & 170 & 160 & 150 & 140 & 130 & 120 & 110 \\ \mathrm{fl}(\mathrm{ppm}) & 100\end{array}$ 
2-((2R,3R)-5-Methyl-3-(quinolin-4-yl)-2,3,4,5-tetrahydro-[1,1'-biphenyl]-2yl)acetaldehyde (3i') [ $\left.{ }^{1} \mathrm{H}_{-} \mathrm{NMR} \_400 \mathrm{MHz}\left(\mathrm{CDCl}_{3}: 7.26 \mathrm{ppm}\right)\right]$
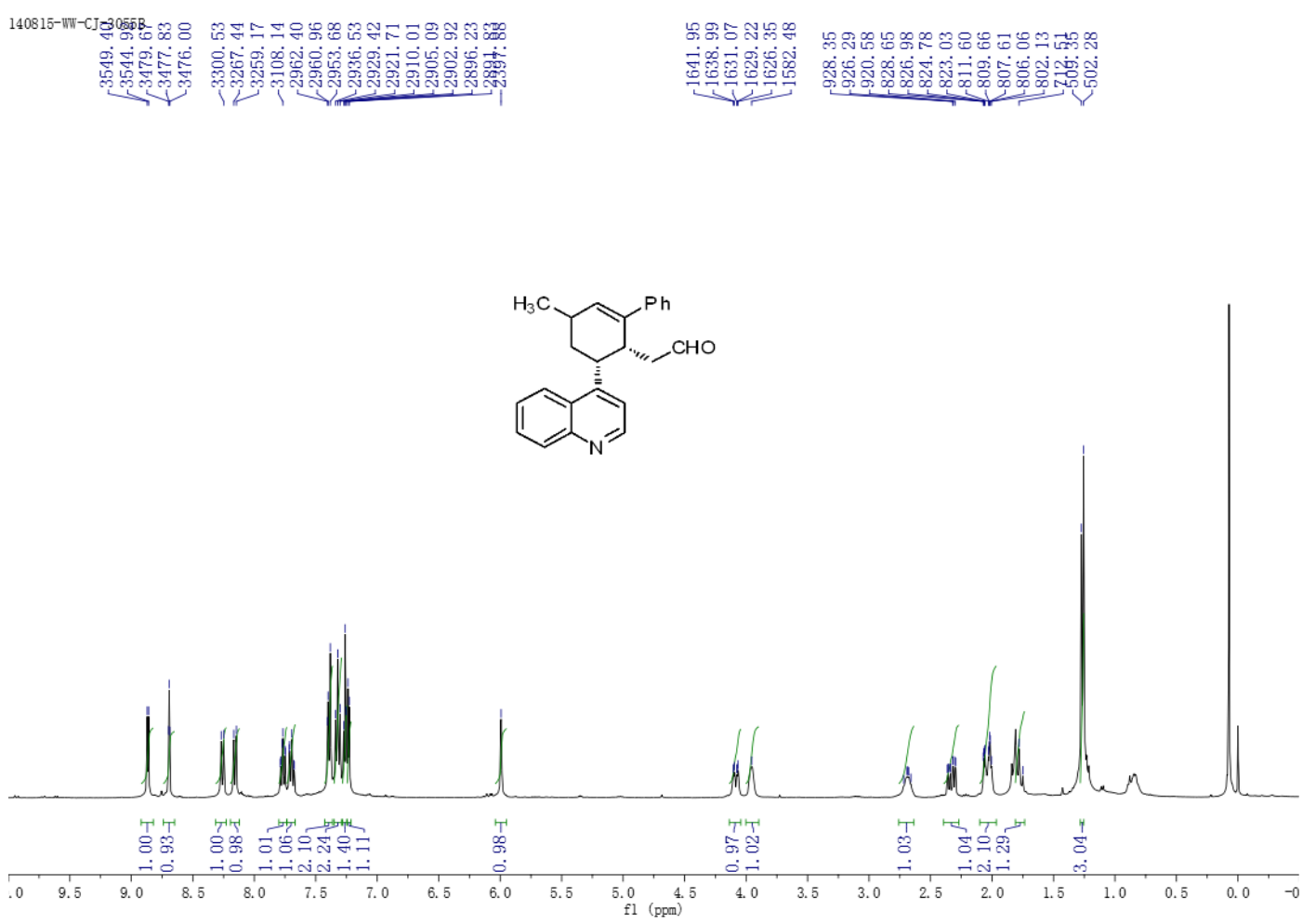

${ }^{13} \mathrm{C} \_N M R \_100 \mathrm{MHz}\left(\mathrm{CDCl}_{3}: 77.00 \mathrm{ppm}\right)$

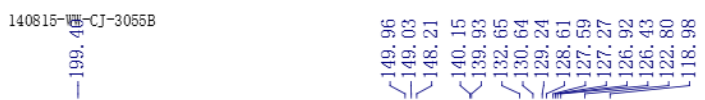

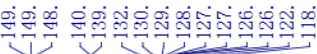
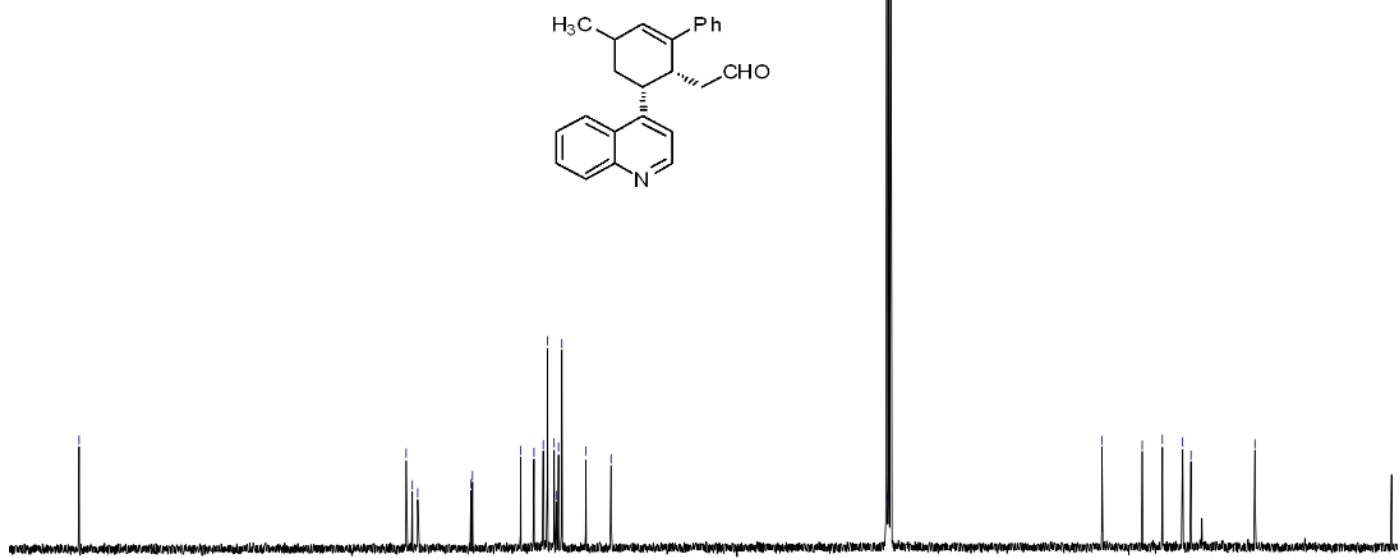

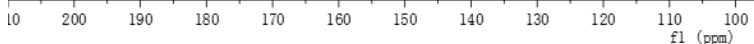


2-((1R,6S)-2-Ethyl-6-(quinolin-4-yl)cyclohex-2-en-1-yl)acetaldehyde (3j) [ ${ }^{1} \mathrm{H} \_\mathrm{NMR} 400$ $\left.\mathrm{MHz}\left(\mathrm{CDCl}_{3}: 7.26 \mathrm{ppm}\right)\right]$

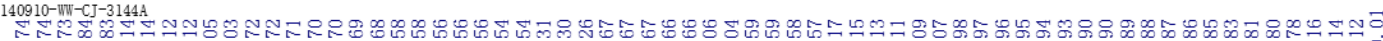

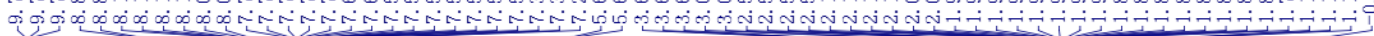

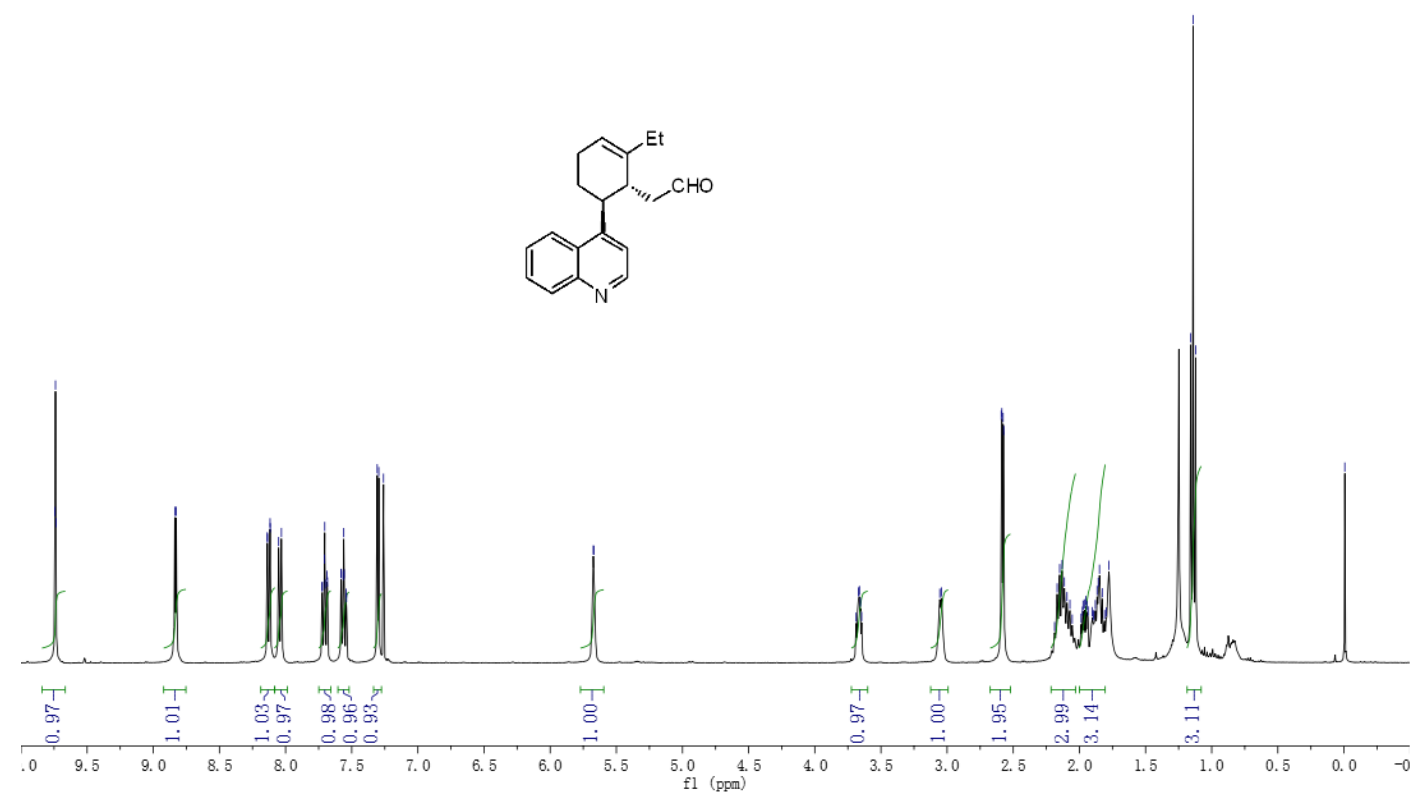

${ }^{13} \mathrm{C} \_\mathrm{NMR} \_100 \mathrm{MHz}\left(\mathrm{CDCl}_{3}: 77.00 \mathrm{ppm}\right)$

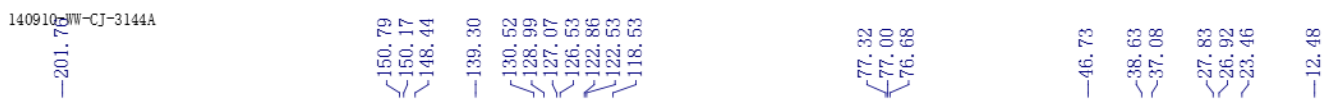<smiles>CCC1=CCCC(c2ccnc3ccccc23)C1CC=O</smiles>

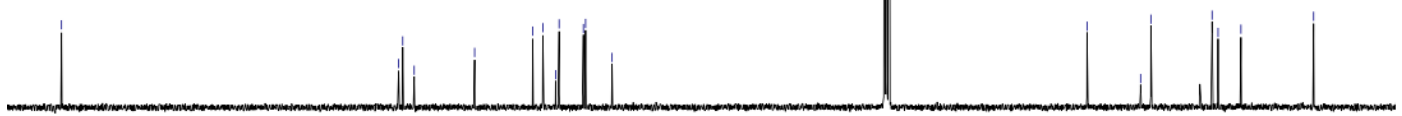

$\begin{array}{lllllllllll}10 & 200 & 190 & 180 & 170 & 160 & 150 & 140 & 130 & 120 & 110 \\ \mathrm{fl}(\mathrm{ppm})\end{array}$ 
2-((1R,6R)-2-Ethyl-6-(quinolin-4-yl)cyclohex-2-en-1-yl)acetaldehyde (3j') [ ${ }^{1}{ }^{H} \_N M R \_400$ $\left.\mathrm{MHz} \_\left(\mathrm{CDCl}_{3}: 7.26 \mathrm{ppm}\right)\right]$

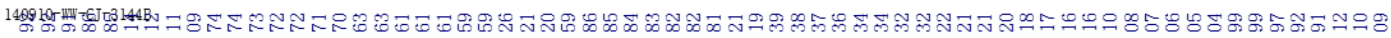

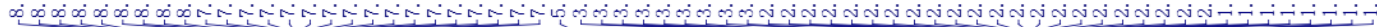

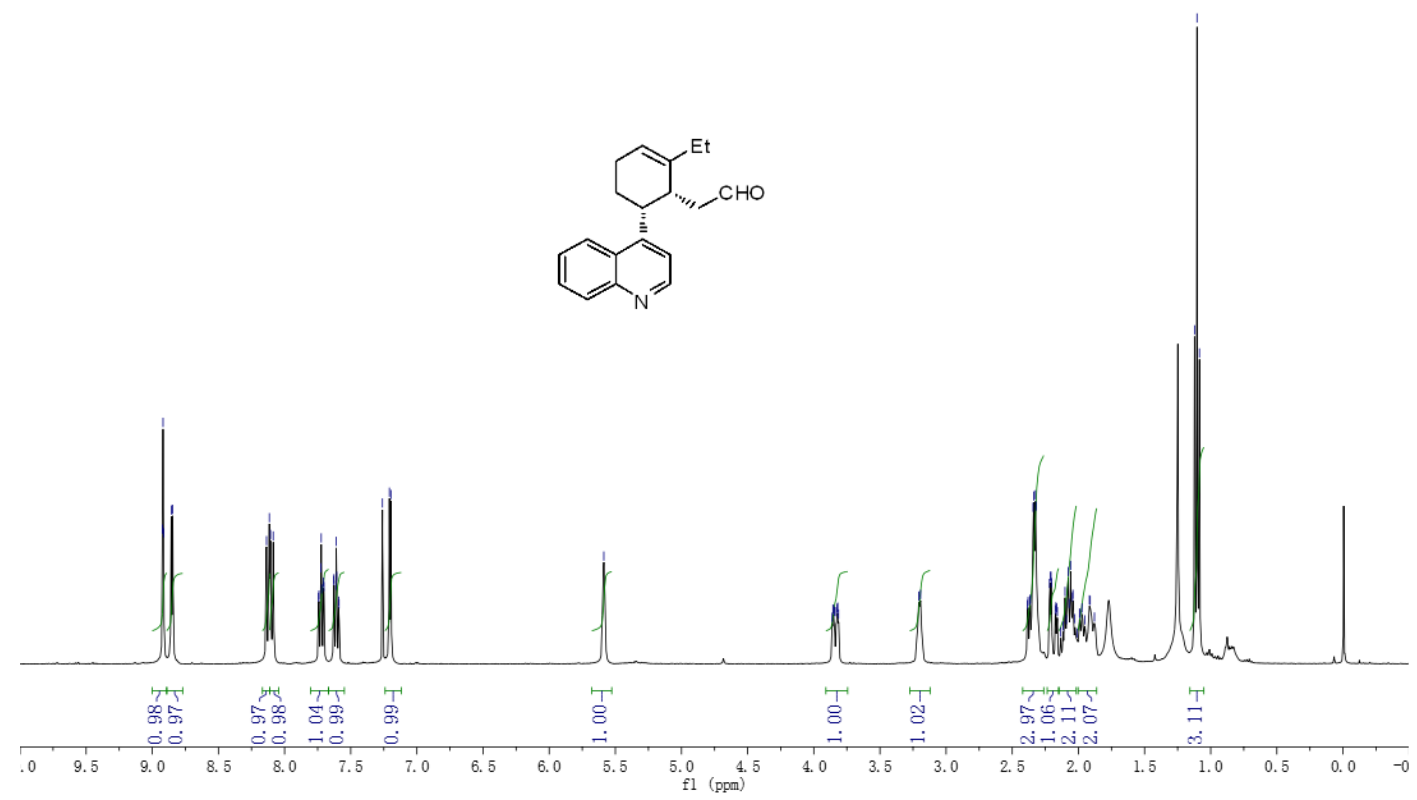

${ }^{13} \mathrm{C} \_\mathrm{NMR} \_100 \mathrm{MHz}\left(\mathrm{CDCl}_{3}: 77.00 \mathrm{ppm}\right)$

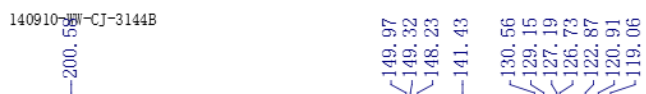

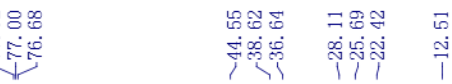
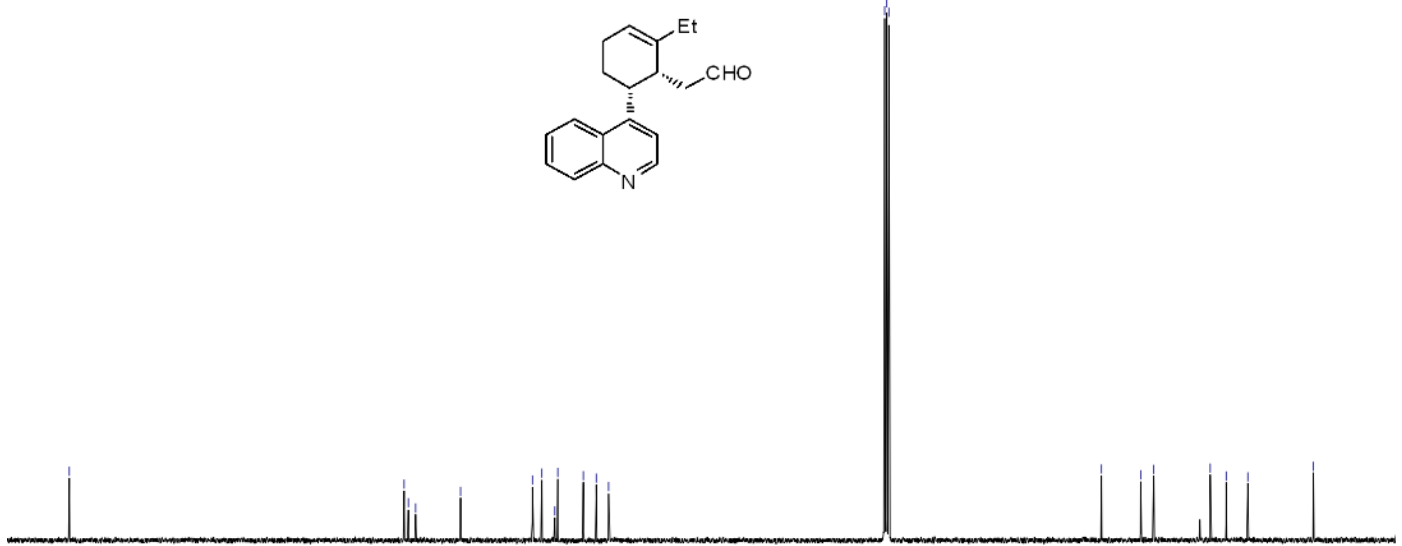

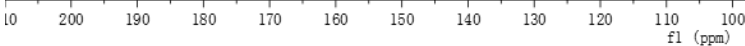


2-((2R,3S)-3-(4-Phenylquinolin-2-yl)-2,3,4,5-tetrahydro-[1,1'-biphenyl]-2yl)acetaldehyde (5) $\left[{ }^{1} \mathrm{H}_{-} \mathrm{NMR} \_400 \mathrm{MHz}\left(\mathrm{CDCl}_{3}: 7.26 \mathrm{ppm}\right)\right]$

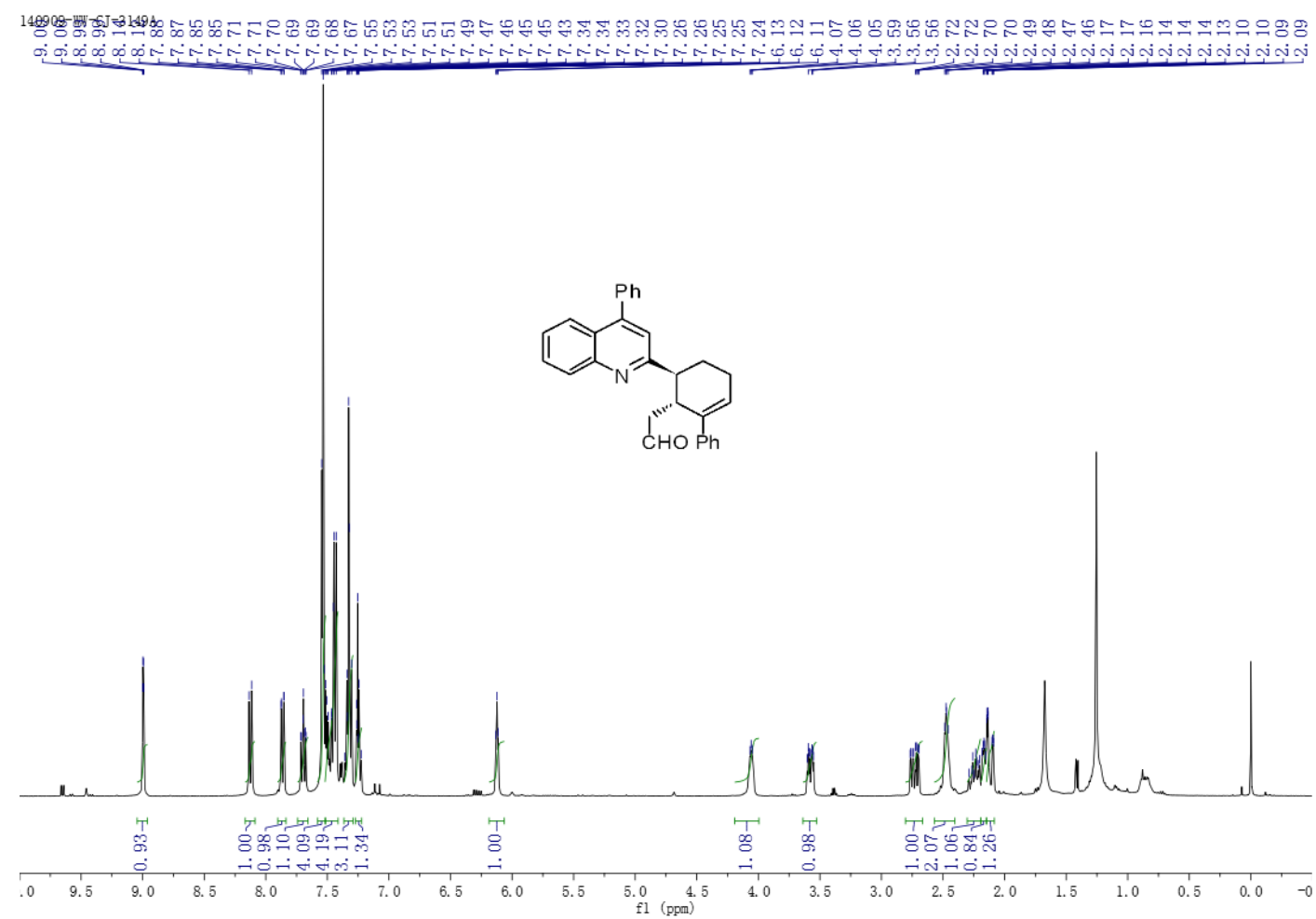

${ }^{13} \mathrm{C} \_\mathrm{NMR} \_100 \mathrm{MHz}\left(\mathrm{CDCl}_{3}: 77.00 \mathrm{ppm}\right)$

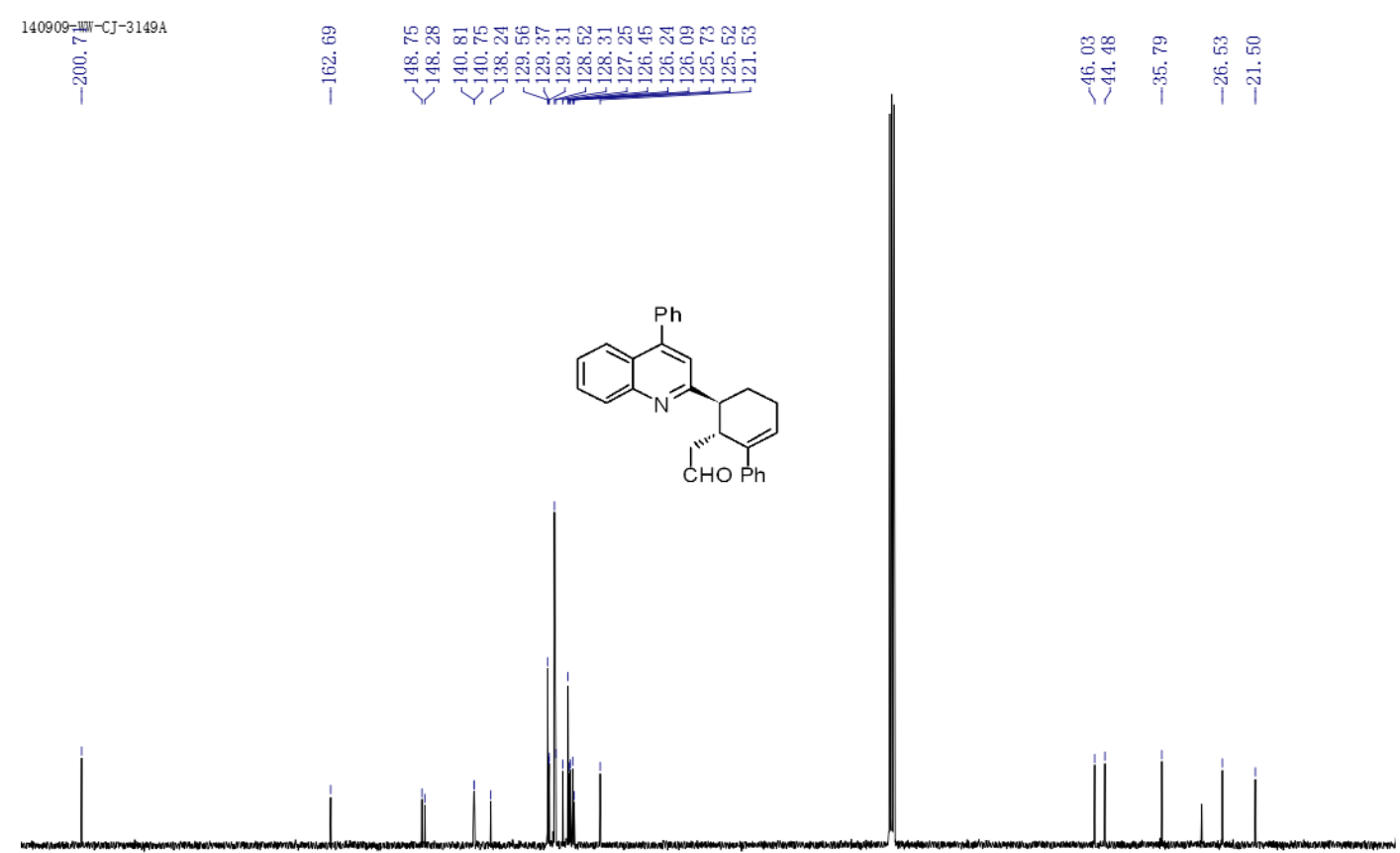

\begin{tabular}{llllllllllll}
\hline 10 & 200 & 190 & 180 & 170 & 160 & 150 & 140 & 130 & 120 & 110 & 100 \\
$\mathrm{fl} 1(\mathrm{ppm})$ & 1
\end{tabular} 


\section{2-((2R,3R)-3-(4-Phenylquinolin-2-yl)-2,3,4,5-tetrahydro-[1,1'-biphenyl]-2-}

yl)acetaldehyde (5') $\left[{ }^{1} \mathrm{H}_{-} \mathrm{NMR} \_400 \mathrm{MHz}\left(\mathrm{CDCl}_{3}: 7.26 \mathrm{ppm}\right)\right]$

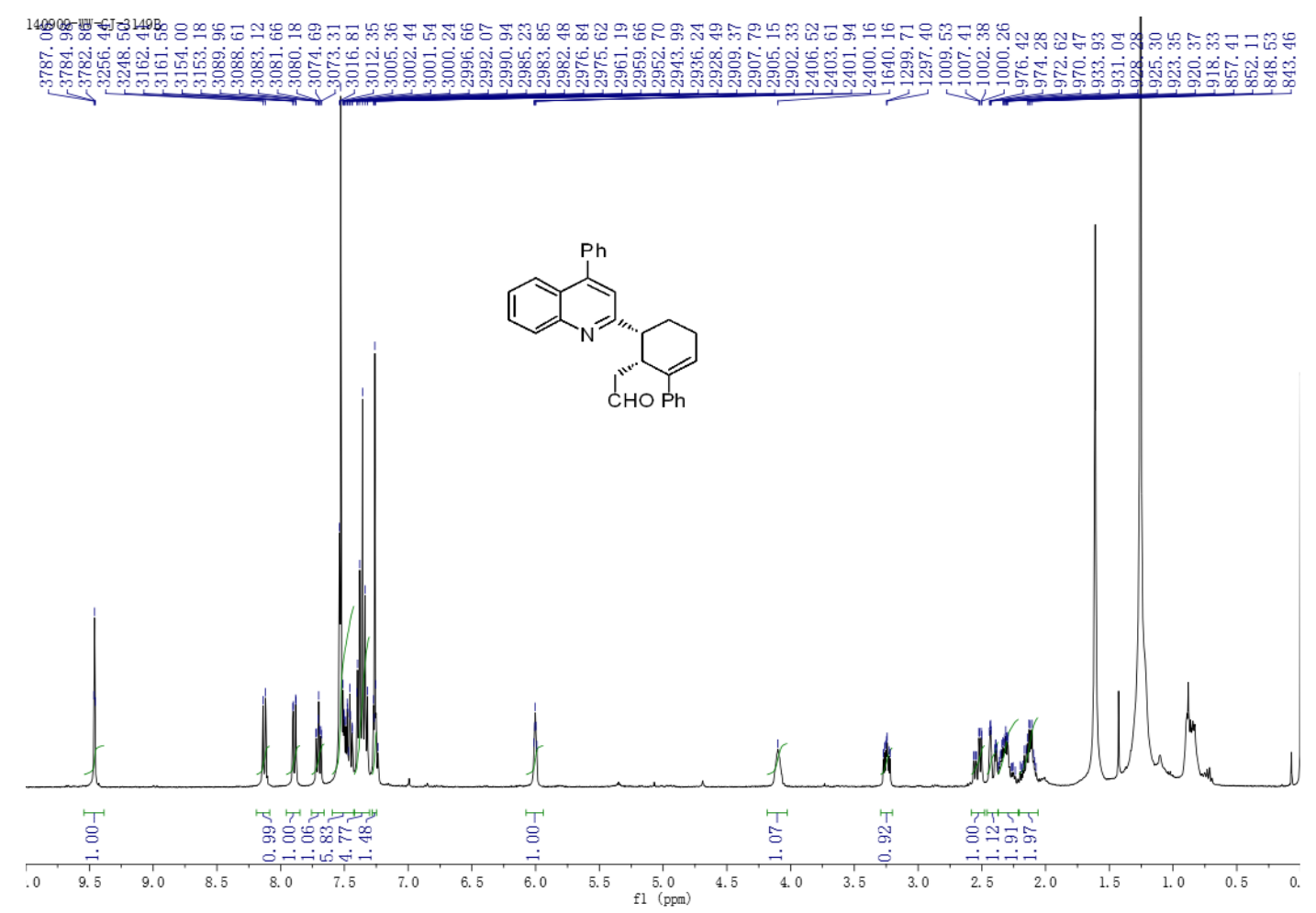

${ }^{13} \mathrm{C} \_N M R \_100 \mathrm{MHz}\left(\mathrm{CDCl}_{3}: 77.00 \mathrm{ppm}\right)$

\begin{tabular}{|c|c|c|c|c|}
\hline 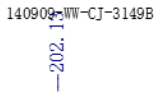 & 象 & 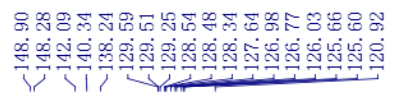 & 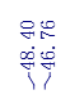 & $\begin{array}{l}8 \\
\dot{0} \\
\dot{0}\end{array}$ \\
\hline
\end{tabular}<smiles>OCCC1C(O)CCCC1c1cc2ccccc2[nH]1</smiles>

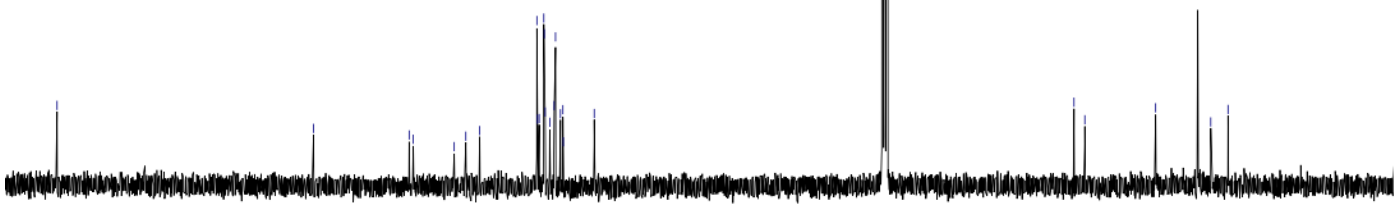

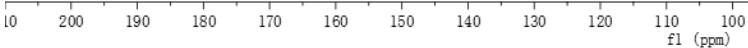




\section{Chiral HPLC analysis trace}

\section{<Chromatogram>}

mAU

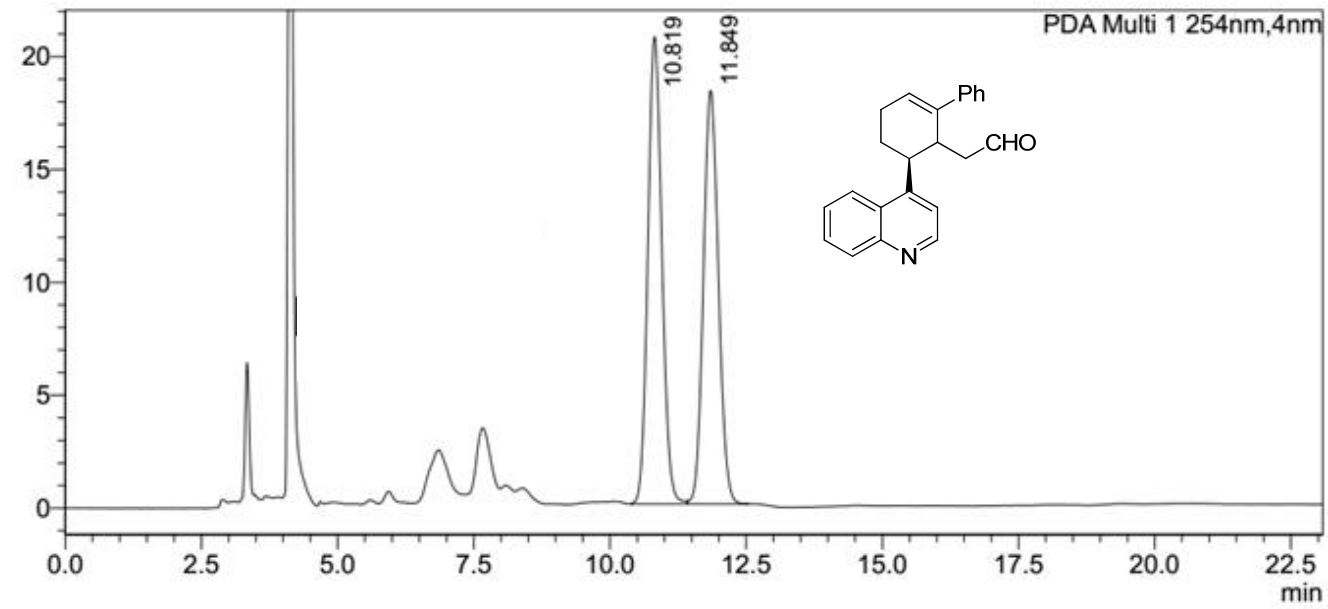

<Peak Table>

PDA Ch1 254nm

\begin{tabular}{|c|c|c|c|c|c|c|c|}
\hline Peak\# & Ret. Time & Area & Height & Conc. & Unit & Mark & Name \\
\hline 1 & 10.819 & 370735 & 19935 & 51.380 & $\%$ & & RT:10.819 \\
\hline 2 & 11.849 & 350822 & 18035 & 48.620 & $\%$ & V & RT:11.849 \\
\hline Total & & 721556 & 37971 & & & & \\
\hline
\end{tabular}

\section{<Chromatogram>}

mAU

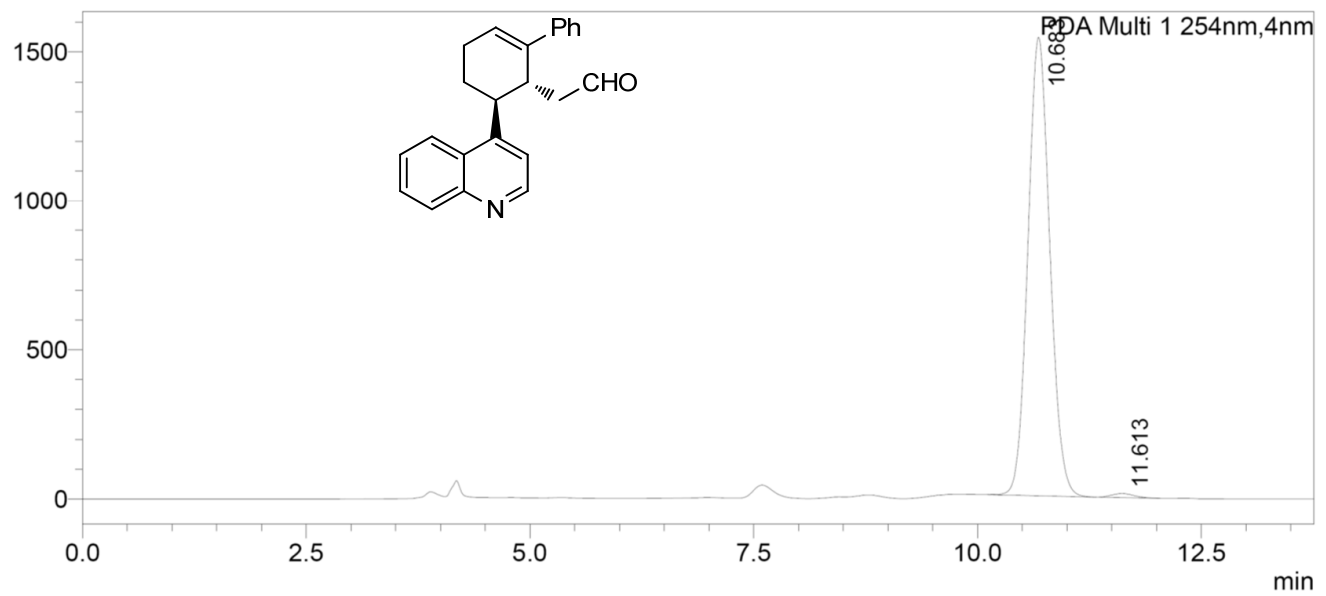

<Peak Table>

PDA Ch1 254nm

Peak\# Ret. Time

\begin{tabular}{ll}
1 & 10.683 \\
\hline
\end{tabular}

\begin{tabular}{|r|r|r|r|r|l|}
\hline \multicolumn{1}{|c|}{ Area } & Height & \multicolumn{1}{|c|}{ Conc. } & Unit & Mark & \multicolumn{2}{|c|}{ Name } \\
\hline 26165479 & 1531687 & 99.145 & $\%$ & & RT: 10.683 \\
\hline 225737 & 13297 & 0.855 & $\%$ & & RT:11.613 \\
\hline 26391216 & 1544984 & & & & \\
\hline
\end{tabular}




\section{$<$ Chromatogram>}

mAU

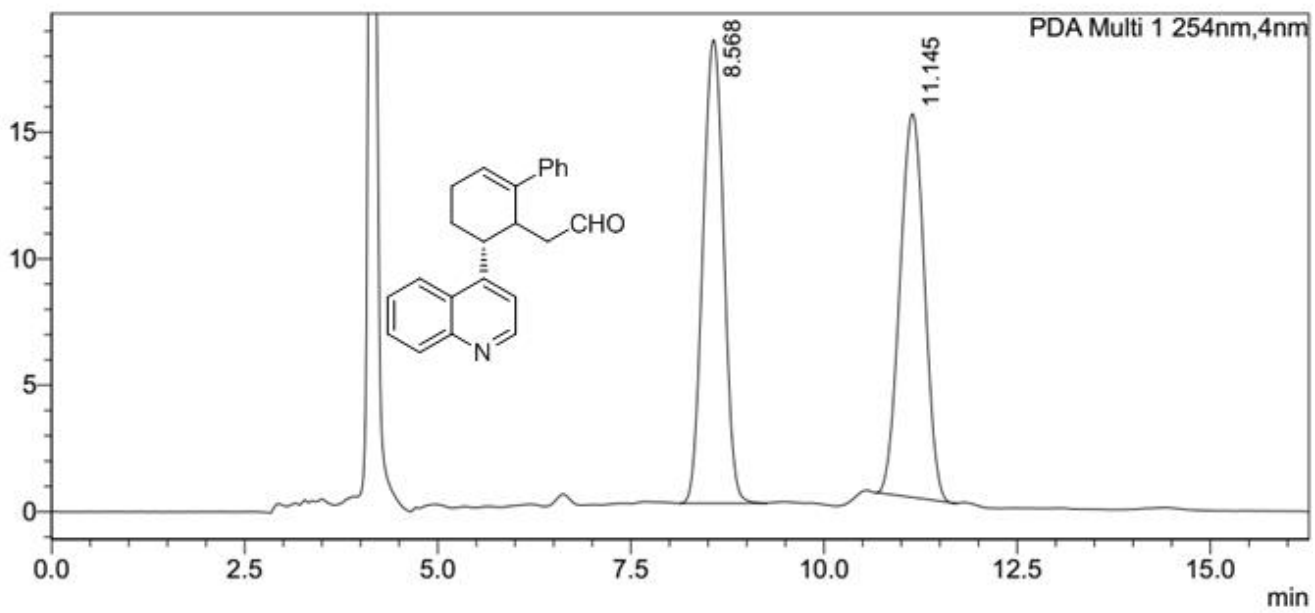

<Peak Table>

PDA Ch1 254nm

\begin{tabular}{|r|r|r|r|r|r|r|l|}
\hline Peak\# & Ret. Time & \multicolumn{1}{|c|}{ Area } & Height & Conc. & Unit & Mark & \multicolumn{2}{|l|}{ Name } \\
\hline 1 & 8.568 & 342421 & 17981 & 50.565 & $\%$ & & RT:8.568 \\
\hline 2 & 11.145 & 334770 & 14846 & 49.435 & $\%$ & & RT: 11.145 \\
\hline Total & & 677191 & 32828 & & & & \\
\hline
\end{tabular}

\section{$<$ Chromatogram >}

mAU

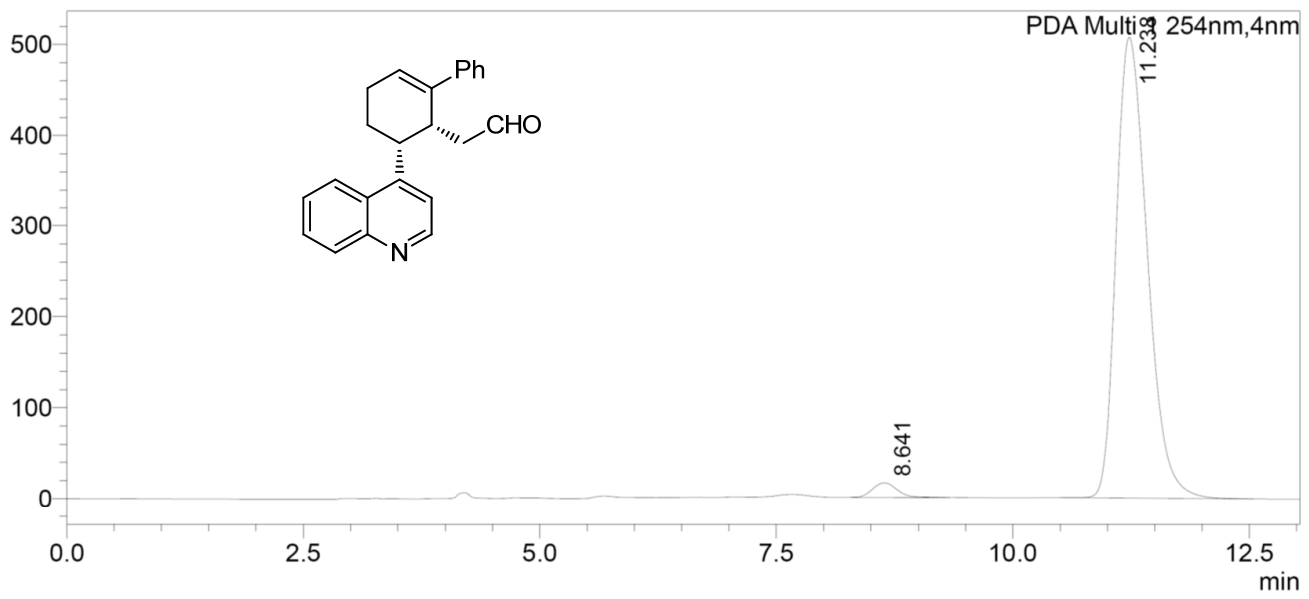

<Peak Table>

PDA Ch1 254nm

Peak\# Ret. Time

\begin{tabular}{|r|r|r|r|r|l|}
\hline \multicolumn{1}{|c|}{ Area } & Height & Conc. & Unit & Mark & \multicolumn{2}{|c|}{ Name } \\
\hline 290655 & 15671 & 2.444 & $\%$ & & RT:8.641 \\
\hline 11603529 & 501361 & $97.556 \%$ & & RT:11.238 \\
\hline 11894183 & 517032 & & & & \\
\hline
\end{tabular}




\section{<Chromatogram>}

MAU

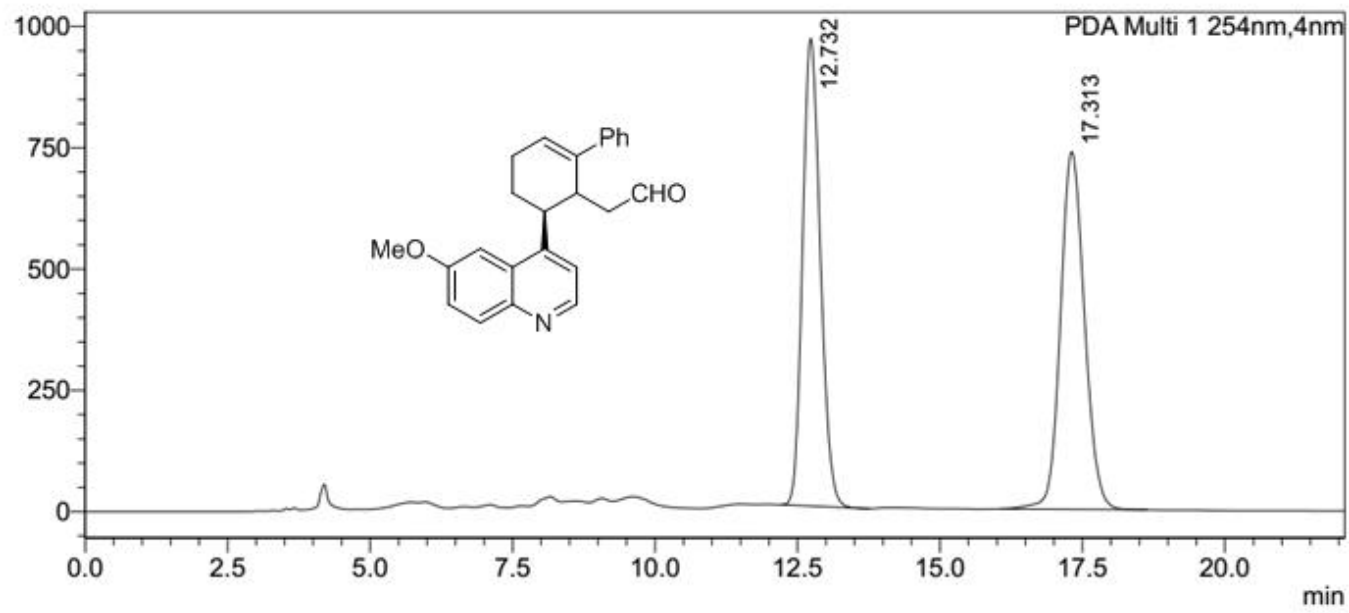

<Peak Table>

PDA Ch1 254nm

\begin{tabular}{|r|r|r|r|r|r|r|l|}
\hline Peak\# & Ret. Time & \multicolumn{1}{c|}{ Area } & Height & Conc. & Unit & Mark & Name \\
\hline 1 & 12.732 & 20877451 & 961091 & 49.158 & $\%$ & & RT:12.732 \\
\hline 2 & 17.313 & 21592869 & 734820 & 50.842 & $\%$ & & RT:17.313 \\
\hline Total & & 42470320 & 1695912 & & & & \\
\hline
\end{tabular}

$<$ Chromatogram $>$

mAU

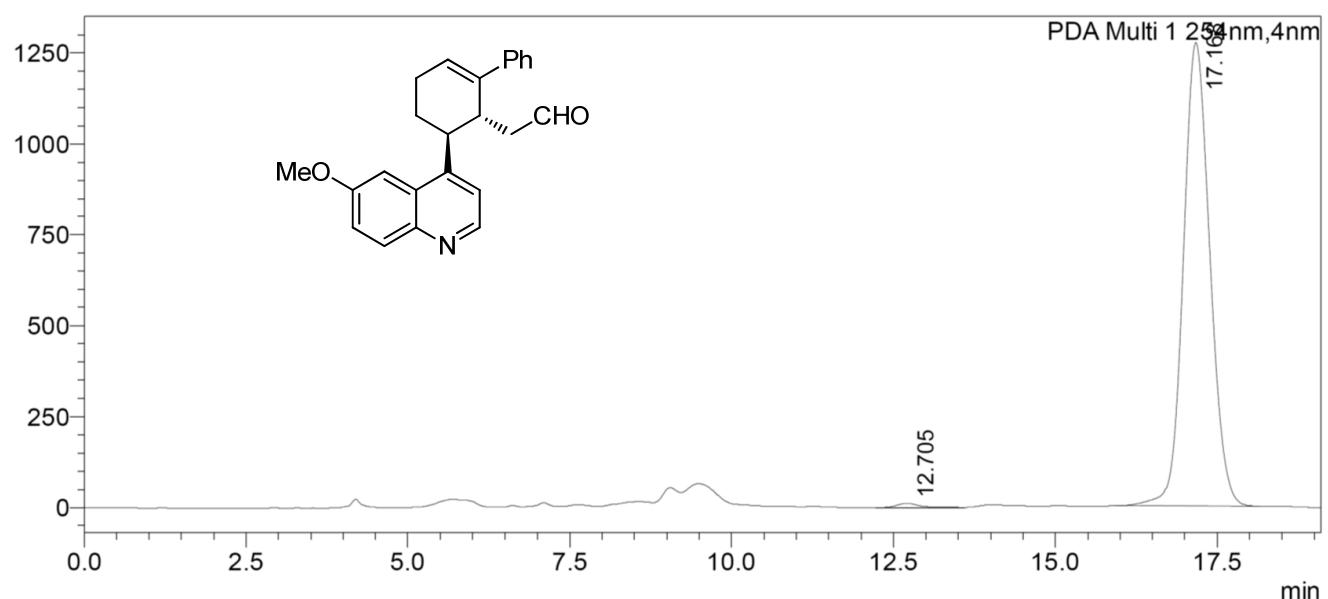

<Peak Table>

PDA Ch1 254nm Peak\# Ret. Time

\begin{tabular}{|c|c|c|c|c|c|c|c|}
\hline Peak\# & et. Time & Area & Height & Conc. & Unit & Mark & Name \\
\hline 1 & 12.705 & 288991 & 11480 & 0.785 & $\%$ & & RT:12.705 \\
\hline 2 & 17.168 & 36502057 & 1271517 & 99.215 & $\%$ & & RT:17.168 \\
\hline Total & & 36791048 & 1282997 & & & & \\
\hline
\end{tabular}


<Chromatogram>

MAU

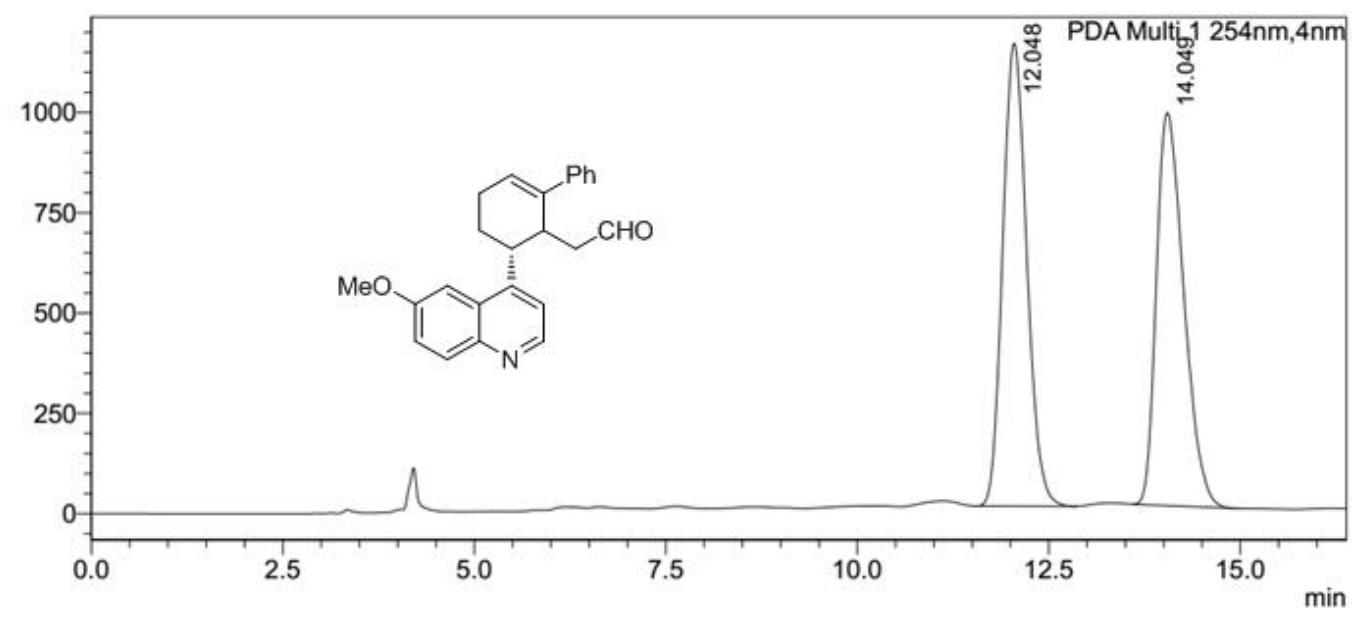

<Peak Table>

PDA Ch1 254nm

\begin{tabular}{|r|r|r|r|r|r|r|l|}
\hline Peak\# & Ret. Time & \multicolumn{1}{|c|}{ Area } & Height & Conc. & Unit & Mark & \multicolumn{2}{|c|}{ Name } \\
\hline 1 & 12.048 & 25171878 & 1150830 & 50.825 & $\%$ & & RT: 12.048 \\
\hline 2 & 14.049 & 24354956 & 975894 & 49.175 & $\%$ & & RT: 14.049 \\
\hline Total & & 49526834 & 2126724 & & & & \\
\hline
\end{tabular}

\section{<Chromatogram>}

UAU

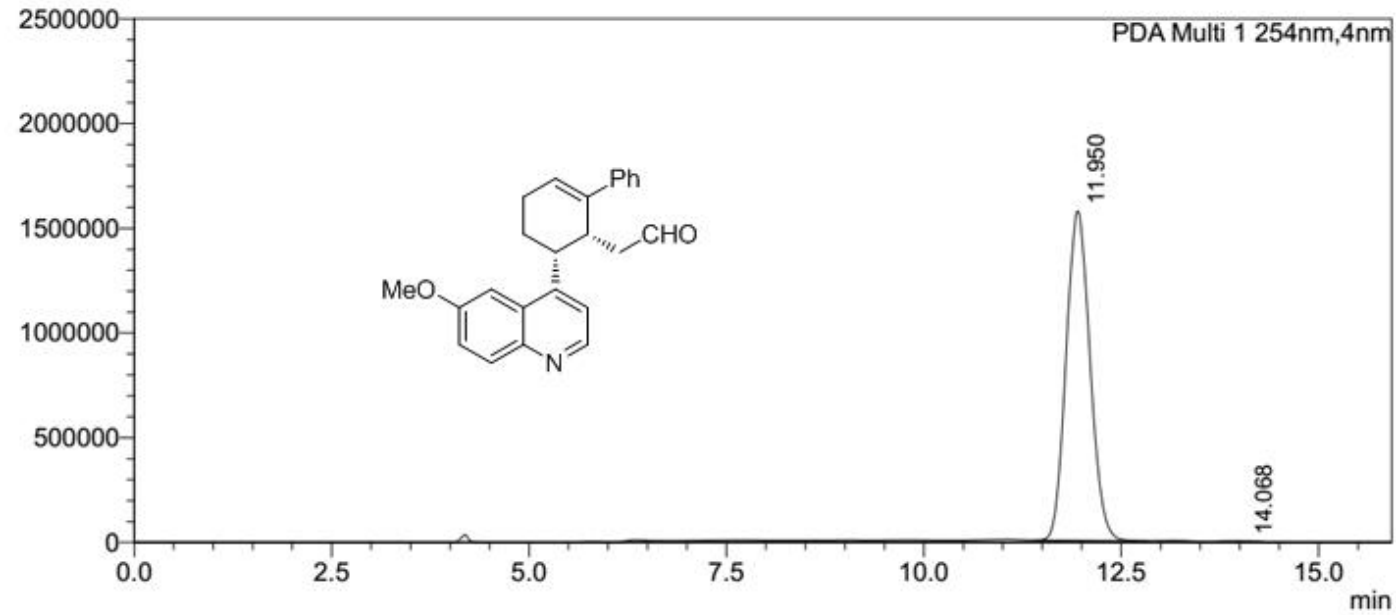

<Peak Table>

PDA Ch1 254nm Peak\# Ret. Time

\begin{tabular}{|c|c|c|c|c|c|c|c|}
\hline Peak\# & Ret. Time & Area & Height & Conc. & Unit & Mark & Name \\
\hline 1 & 11.950 & 33160673 & 1567251 & 99.773 & $\%$ & & RT:11.950 \\
\hline 2 & 14.068 & 75314 & 3720 & 0.227 & $\%$ & & RT:14.068 \\
\hline Total & & 33235988 & 1570971 & & & & \\
\hline
\end{tabular}




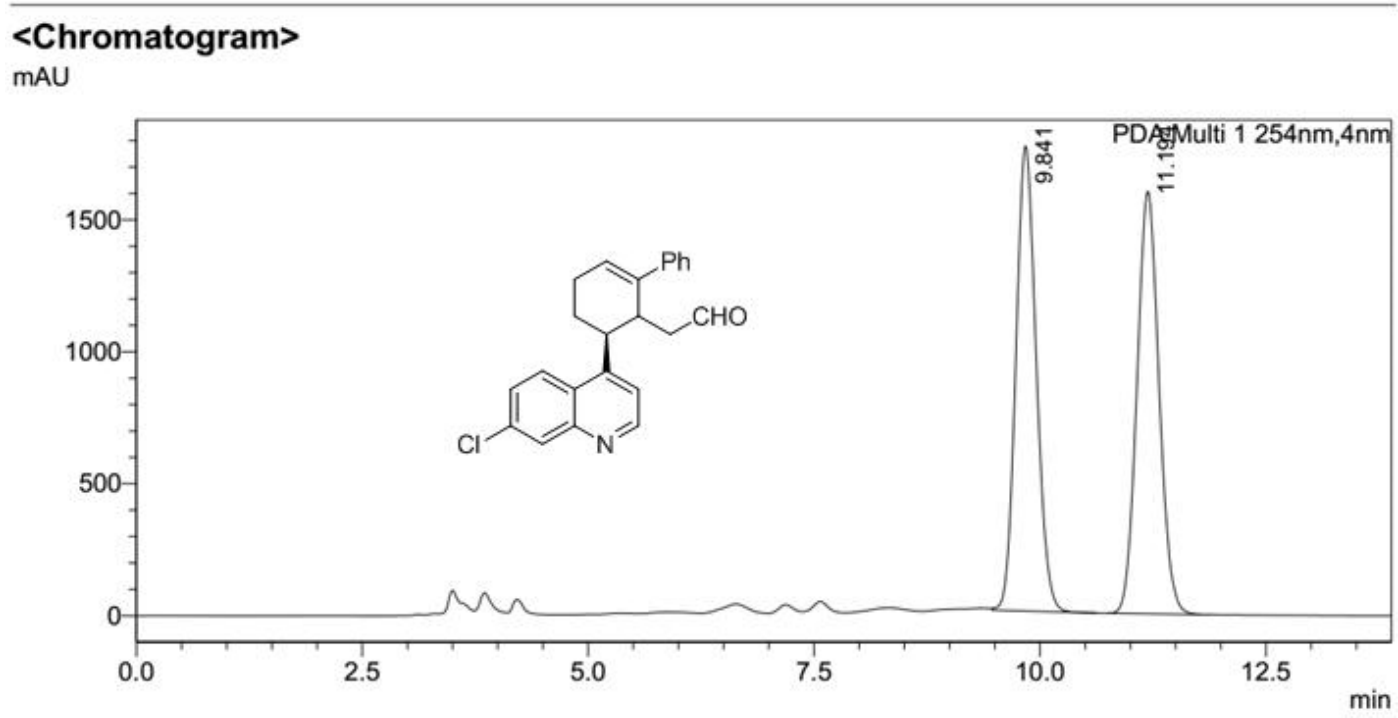

<Peak Table>

PDA Ch1 254nm

\begin{tabular}{|r|r|r|r|r|r|r|l|}
\hline Peak\# & Ret. Time & Area & Height & Conc. & Unit & Mark & \multicolumn{1}{|c|}{ Name } \\
\hline 1 & 9.841 & 27479671 & 1753810 & 50.322 & $\%$ & V & RT:9.841 \\
\hline 2 & 11.194 & 27127895 & 1594422 & 49.678 & $\%$ & & RT:11.194 \\
\hline Total & & 54607566 & 3348232 & & & & \\
\hline
\end{tabular}

\section{<Chromatogram>}

mAU

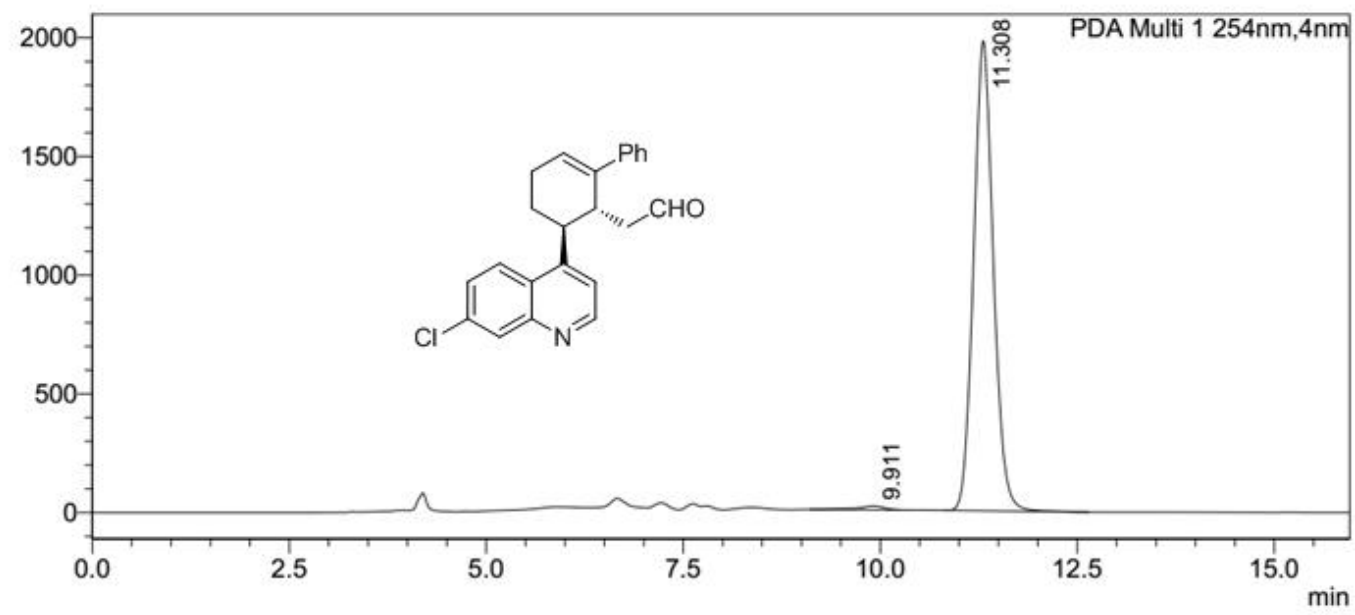

<Peak Table>

PDA Ch1 254nm Peak\# Ret. Time

\begin{tabular}{|c|c|c|c|c|c|c|c|}
\hline Peak\#\# & et. Time & Area & Height & Conc. & Unit & Mark & Name \\
\hline 1 & 9.911 & 415485 & 16821 & 1.189 & $\%$ & & RT:9.911 \\
\hline 2 & 11.308 & 34542954 & 1964145 & 98.811 & $\%$ & & RT:11.308 \\
\hline Total & & 34958439 & 1980966 & & & & \\
\hline
\end{tabular}




\section{$<$ Chromatogram>}

mAU

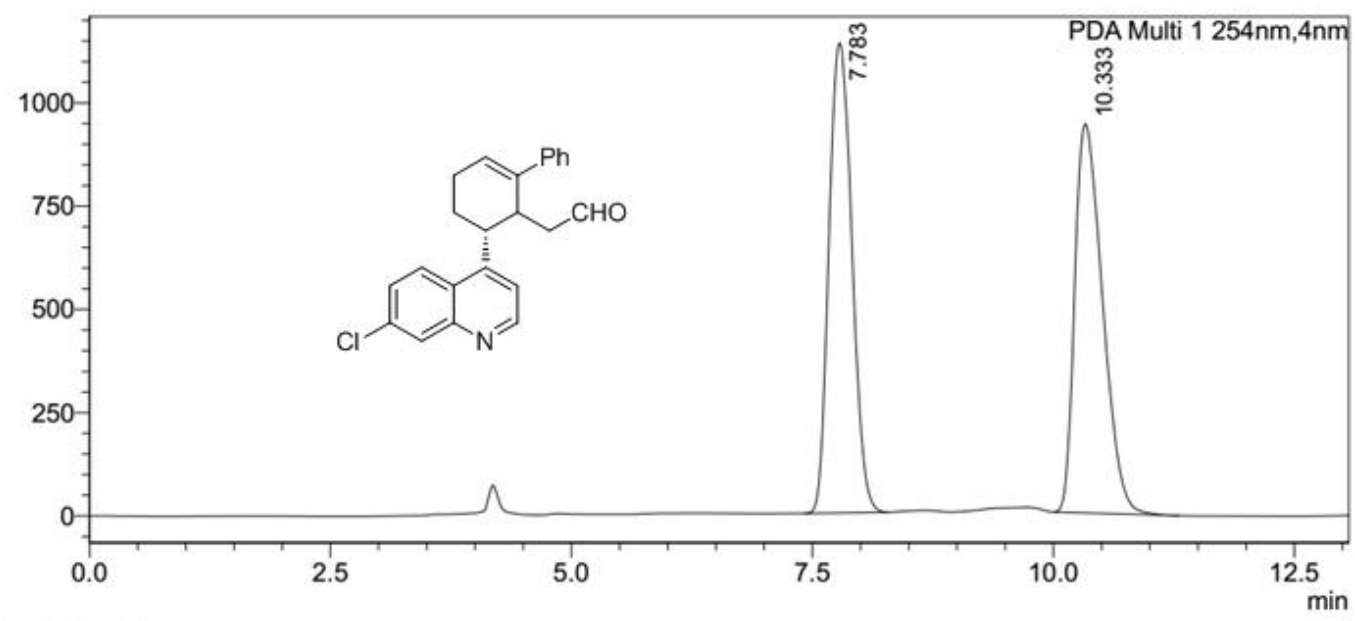

<Peak Table>

PDA Ch1 254nm

\begin{tabular}{|c|c|c|c|c|c|c|c|}
\hline Peak\# & Ret. Time & Area & Height & Conc. & Unit & Mark & Name \\
\hline 1 & 7.783 & 18914928 & 1127489 & 50.037 & $\%$ & & RT:7.783 \\
\hline 2 & 10.333 & 18886677 & 939325 & 49.963 & $\%$ & & RT:10.333 \\
\hline Total & & 37801605 & 2066814 & & & & \\
\hline
\end{tabular}

\section{<Chromatogram>}

$\mathrm{mAU}$

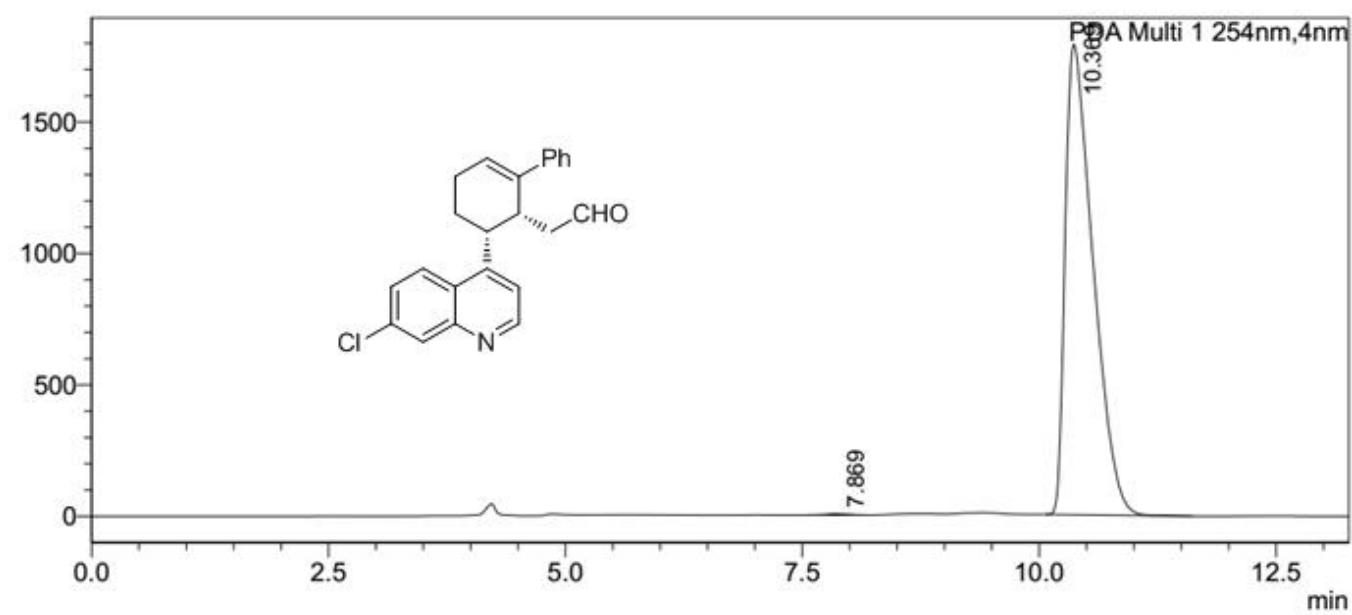

<Peak Table>

PDA Ch1 254nm

\begin{tabular}{rr} 
Peak\# Ret. Time \\
\hline 1 & 7.869
\end{tabular}

\begin{tabular}{|r|r|}
\hline 1 & 7.869 \\
\hline 2 & 10.369 \\
\hline
\end{tabular}

\begin{tabular}{|r|r|r|r|r|}
\hline \multicolumn{1}{|c|}{ Area } & Height & Conc. & Unit & Mark \\
\hline 78622 & 4327 & 0.215 & $\%$ & \\
\hline 36542601 & 1778724 & 99.785 & $\%$ & \\
\hline 36621223 & 1783051 & & & \\
\hline
\end{tabular}

RT:7.869

RT:10.369 


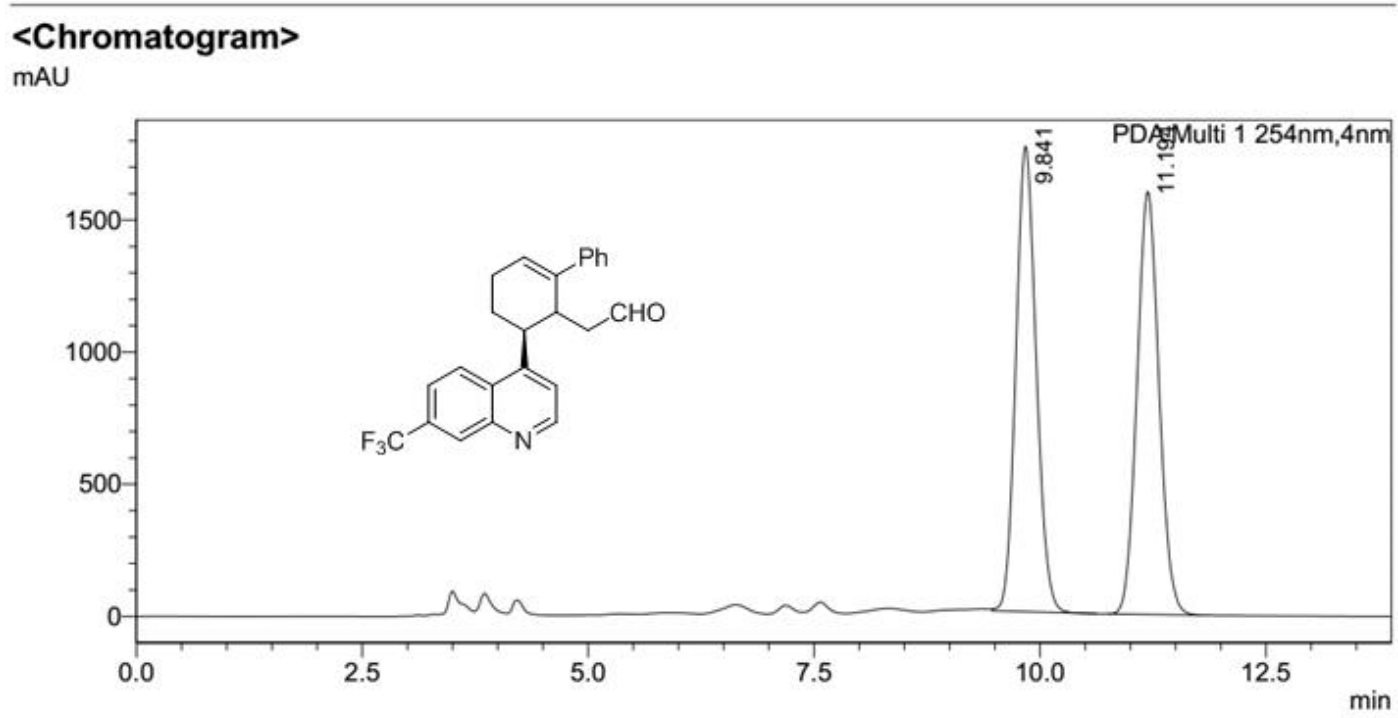

<Peak Table>

PDA Ch1 254nm

\begin{tabular}{|c|c|c|c|c|c|c|c|}
\hline Peak\# & Ret. Time & Area & Height & Conc. & Unit & Mark & Name \\
\hline 1 & 9.841 & 27479671 & 1753810 & 50.322 & $\%$ & V & RT:9.841 \\
\hline 2 & 11.194 & 27127895 & 1594422 & 49.678 & $\%$ & & RT:11.194 \\
\hline Total & & 54607566 & 3348232 & & & & \\
\hline
\end{tabular}

\section{<Chromatogram>}

mAU

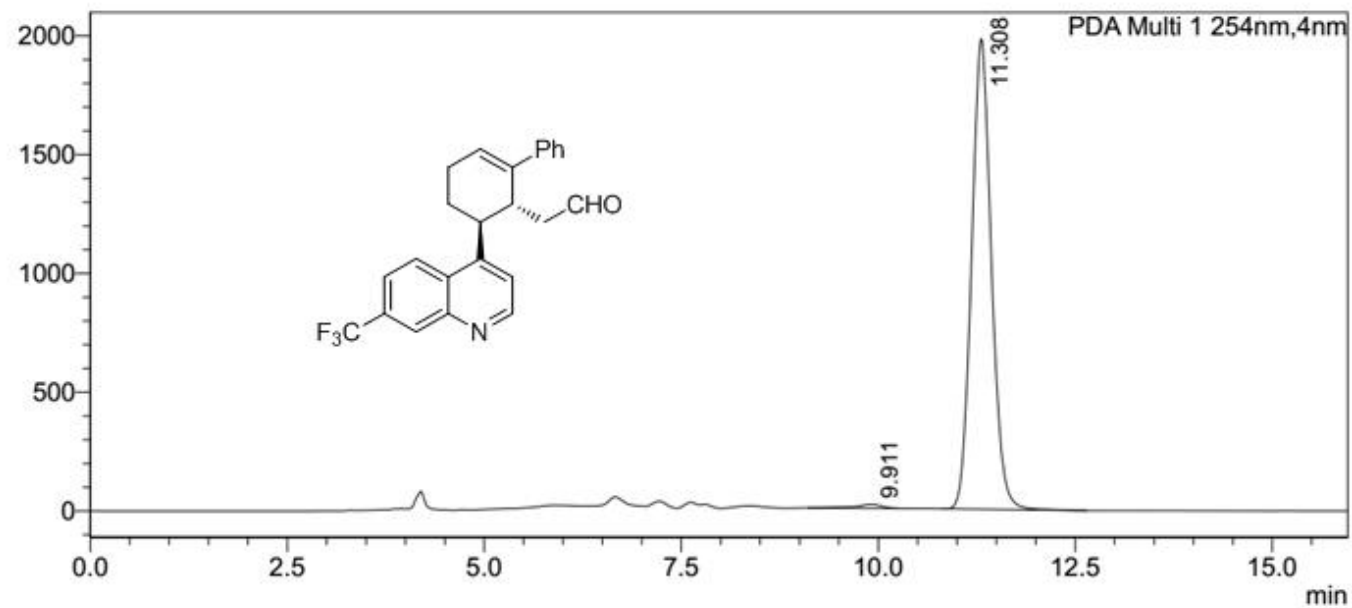

<Peak Table>

PDA Ch1 254nm

Peak\# Ret. Time

\begin{tabular}{|c|c|c|c|c|c|c|c|}
\hline Peak\# & Ret. Time & Area & Height & Conc. & Unit & Mark & Name \\
\hline 1 & 9.911 & 415485 & 16821 & 1.189 & $\%$ & & RT:9.911 \\
\hline 2 & 11.308 & 34542954 & 1964145 & 98.811 & $\%$ & & RT:11.308 \\
\hline Total & & 34958439 & 1980966 & & & & \\
\hline
\end{tabular}




\section{$<$ Chromatogram>}

mAU

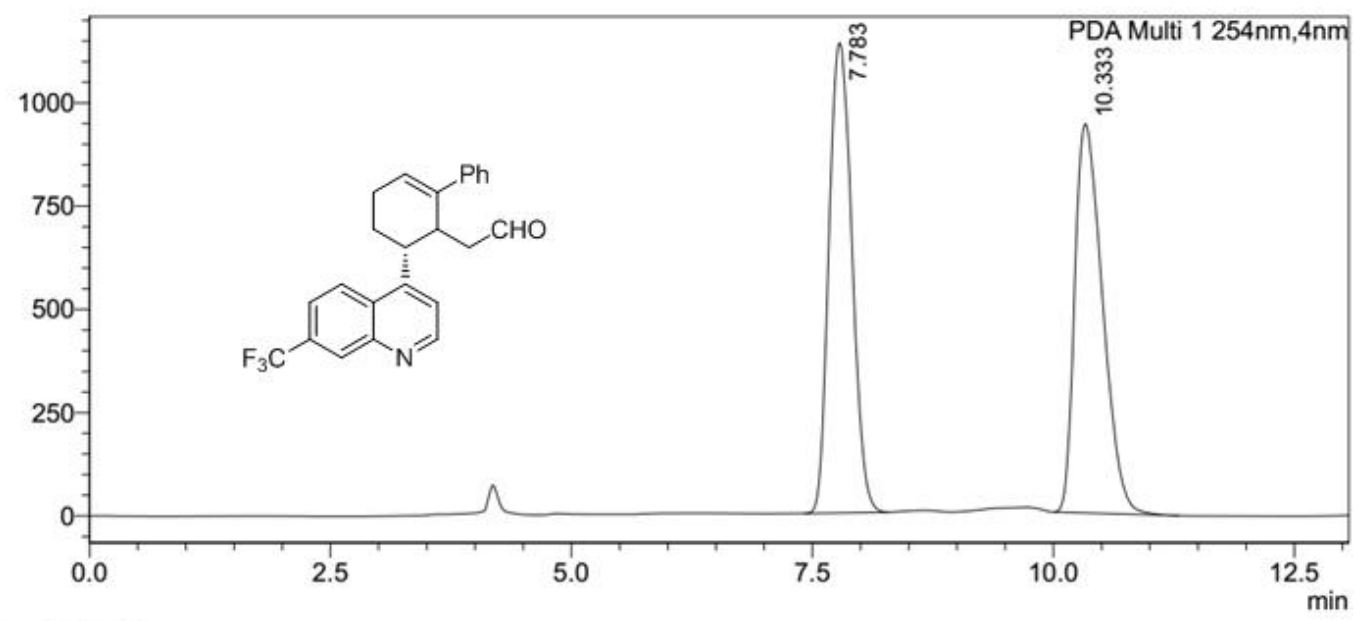

<Peak Table>

PDA Ch1 254nm

\begin{tabular}{|c|c|c|c|c|c|c|c|}
\hline Peak\# & Ret. Time & Area & Height & Conc. & Unit & Mark & Name \\
\hline 1 & 7.783 & 18914928 & 1127489 & 50.037 & $\%$ & & RT:7.783 \\
\hline 2 & 10.333 & 18886677 & 939325 & 49.963 & $\%$ & & RT:10.333 \\
\hline Total & & 37801605 & 2066814 & & & & \\
\hline
\end{tabular}

\section{<Chromatogram>}

$\mathrm{mAU}$

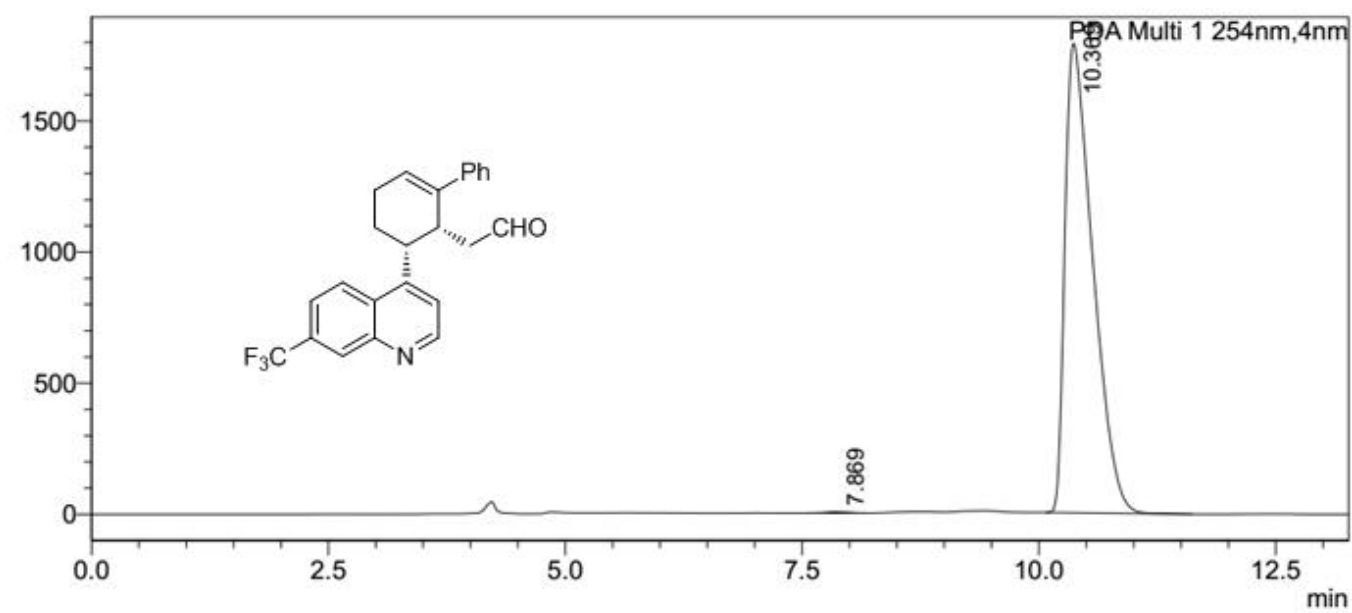

<Peak Table>

PDA Ch1 254nm

\begin{tabular}{|r|r|}
\hline Peak\# & Ret. Time \\
\hline 1 & 7.869 \\
\hline
\end{tabular}

\begin{tabular}{|c|c|c|c|c|c|c|c|}
\hline Peak\# & Ret. Time & Area & Height & Conc. & Unit & Mark & Name \\
\hline 1 & 7.869 & 78622 & 4327 & 0.215 & $\%$ & & RT:7.869 \\
\hline 2 & 10.369 & 36542601 & 1778724 & 99.785 & $\%$ & & RT:10.369 \\
\hline Total & & 36621223 & 1783051 & & & & \\
\hline
\end{tabular}




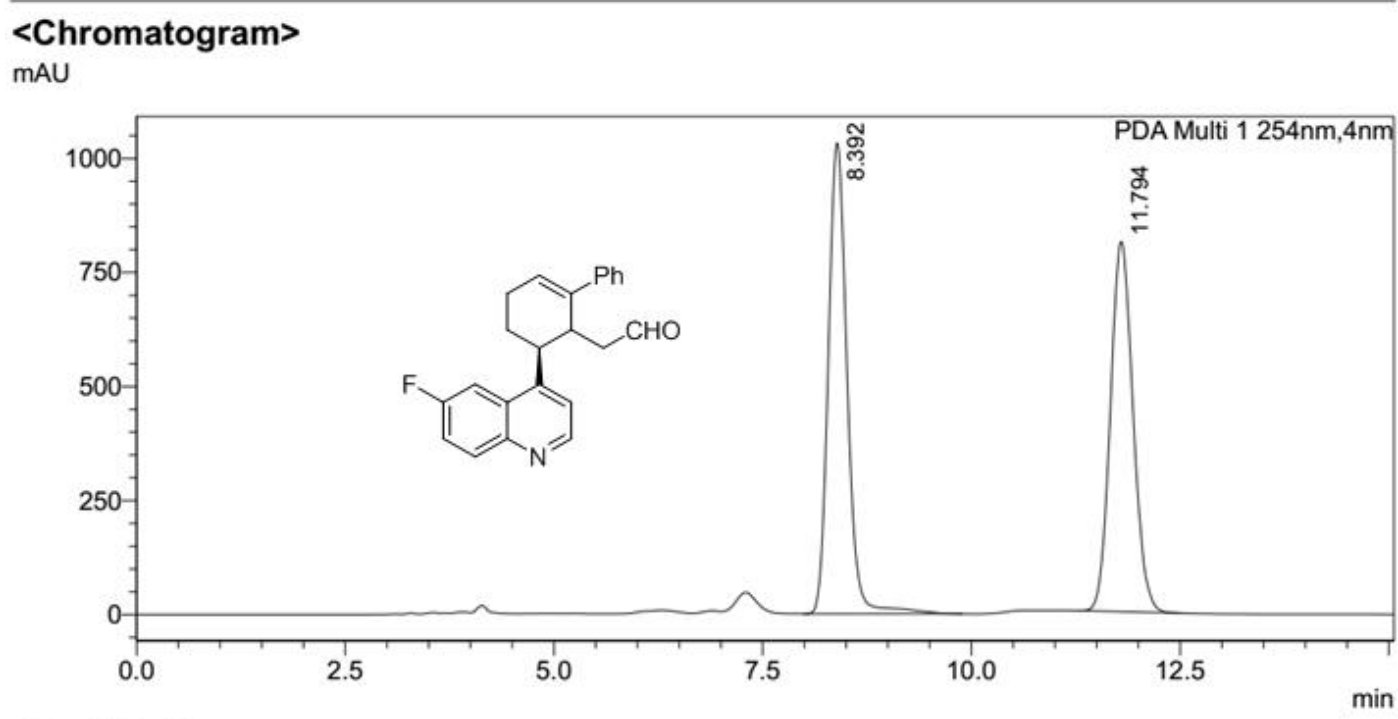

<Peak Table>

PDA Ch1 254nm

\begin{tabular}{|r|r|r|r|r|r|r|l|}
\hline Peak\# & Ret. Time & \multicolumn{1}{|c|}{ Area } & \multicolumn{1}{c|}{ Height } & Conc. & Unit & Mark & \multicolumn{2}{|c|}{ Name } \\
\hline 1 & 8.392 & 16153748 & 1019073 & 51.377 & $\%$ & & RT:8.392 \\
\hline 2 & 11.794 & 15287733 & 809123 & 48.623 & $\%$ & & RT:11.794 \\
\hline Total & & 31441481 & 1828195 & & & & \\
\hline
\end{tabular}

\section{<Chromatogram>}

mAU

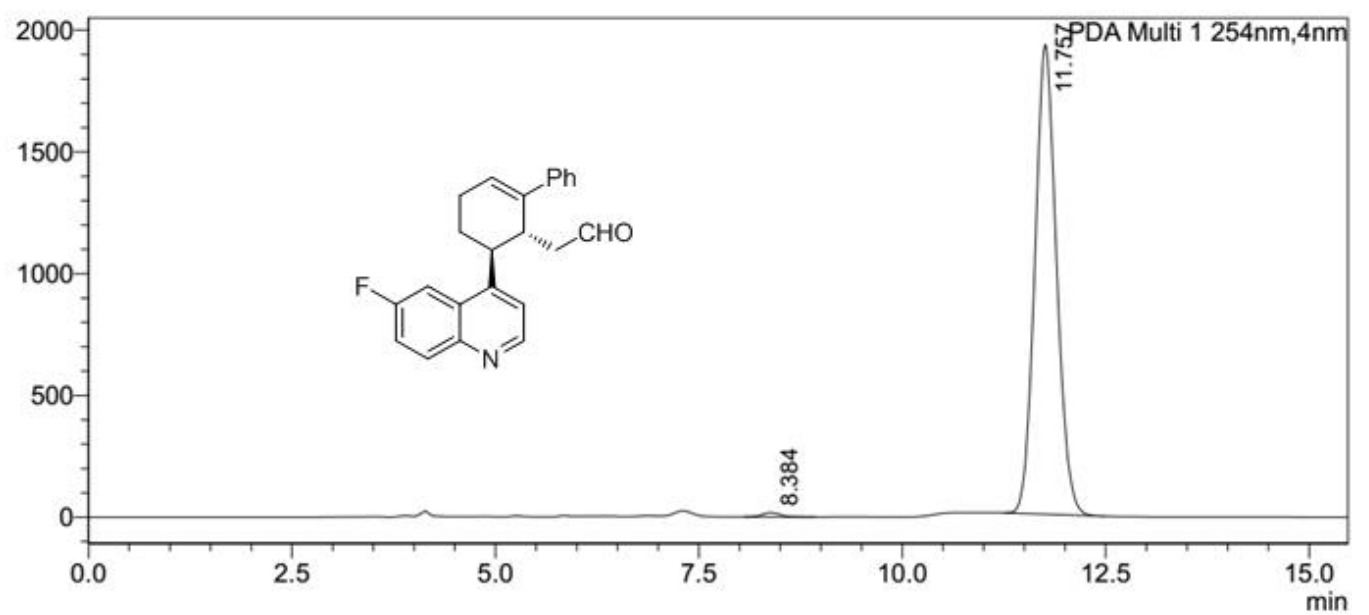

<Peak Table>

PDA Ch1 254nm

Peak\# Ret. Time

\begin{tabular}{|r|r|r|r|r|r|r|l|}
\hline Peak\# & Ret. Time & \multicolumn{1}{|c|}{ Area } & \multicolumn{1}{c|}{ Height } & \multicolumn{1}{c|}{ Conc. } & Unit & Mark & \multicolumn{2}{|c|}{ Name } \\
\hline 1 & 8.384 & 271098 & 17606 & 0.758 & $\%$ & & RT:8.384 \\
\hline 2 & 11.757 & 35494261 & 1917053 & 99.242 & $\%$ & & RT:11.757 \\
\hline Total & & 35765359 & 1934659 & & & & \\
\hline
\end{tabular}


$<$ Chromatogram>

mAU

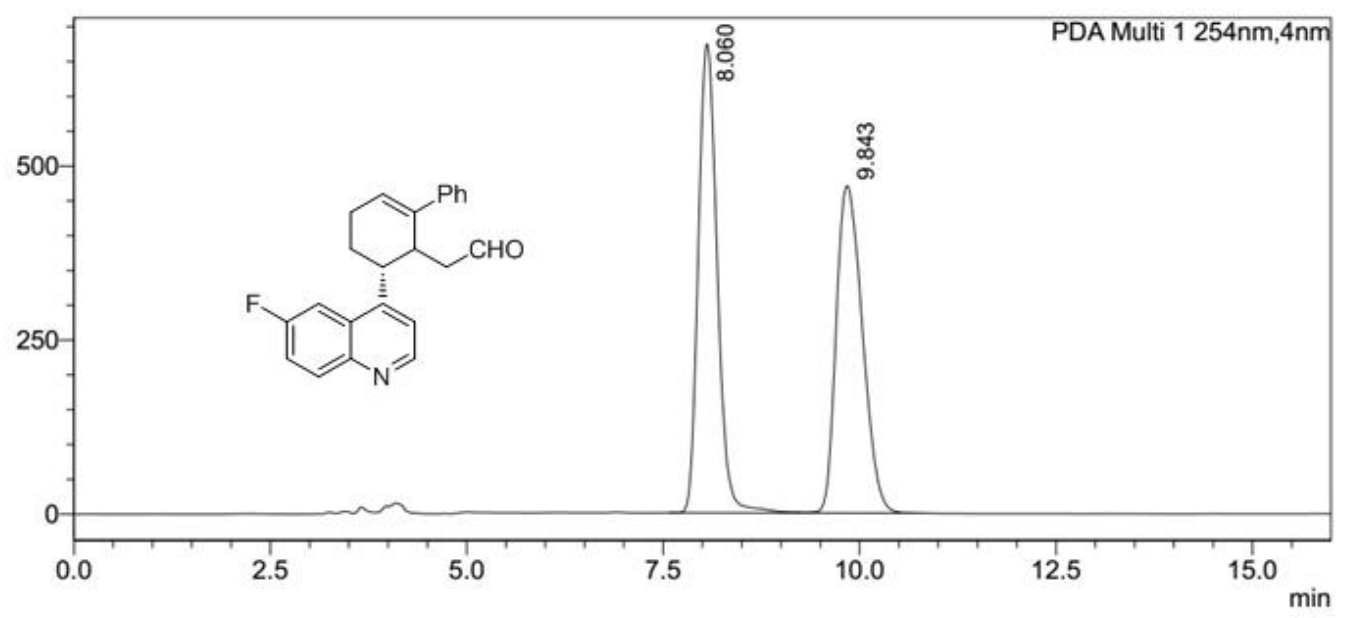

<Peak Table>

PDA Ch1 254nm

\begin{tabular}{|r|r|r|r|r|r|r|r|}
\hline Peak\# & Ret. Time & \multicolumn{1}{c|}{ Area } & Height & Conc. & Unit & Mark & Name \\
\hline 1 & 8.060 & 11264734 & 670928 & 50.839 & $\%$ & & RT:8.060 \\
\hline 2 & 9.843 & 10892906 & 469189 & 49.161 & $\%$ & V & RT:9.843 \\
\hline Total & & 22157640 & 1140117 & & & & \\
\hline
\end{tabular}

\section{<Chromatogram>}

mAU

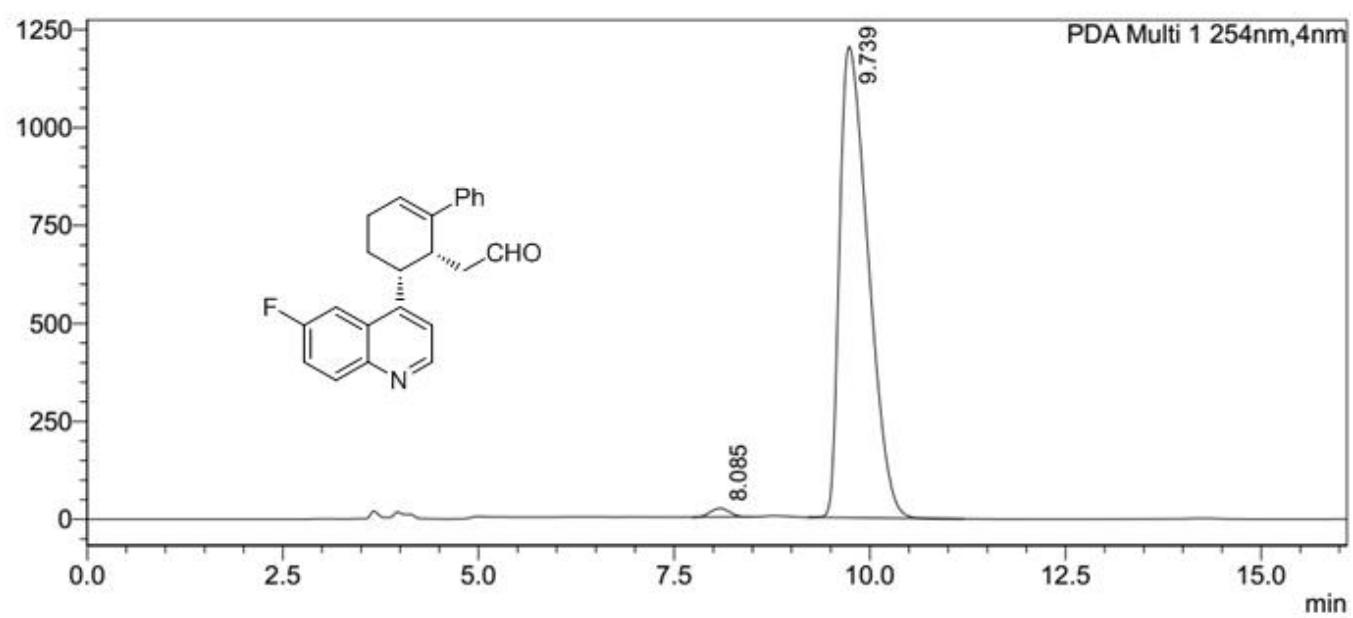

<Peak Table>

PDA Ch1 254nm

Peak\# Ret. Time

\begin{tabular}{|c|c|c|c|c|c|c|c|}
\hline Peak\# & Ret. Time & Area & Height & Conc. & Unit & Mark & Name \\
\hline 1 & 8.085 & 372032 & 21866 & 1.204 & $\%$ & & RT:8.085 \\
\hline 2 & 9.739 & 30530978 & 1198356 & 98.796 & $\%$ & & RT:9.739 \\
\hline Total & & 30903010 & 1220222 & & & & \\
\hline
\end{tabular}


<Chromatogram>

mAU

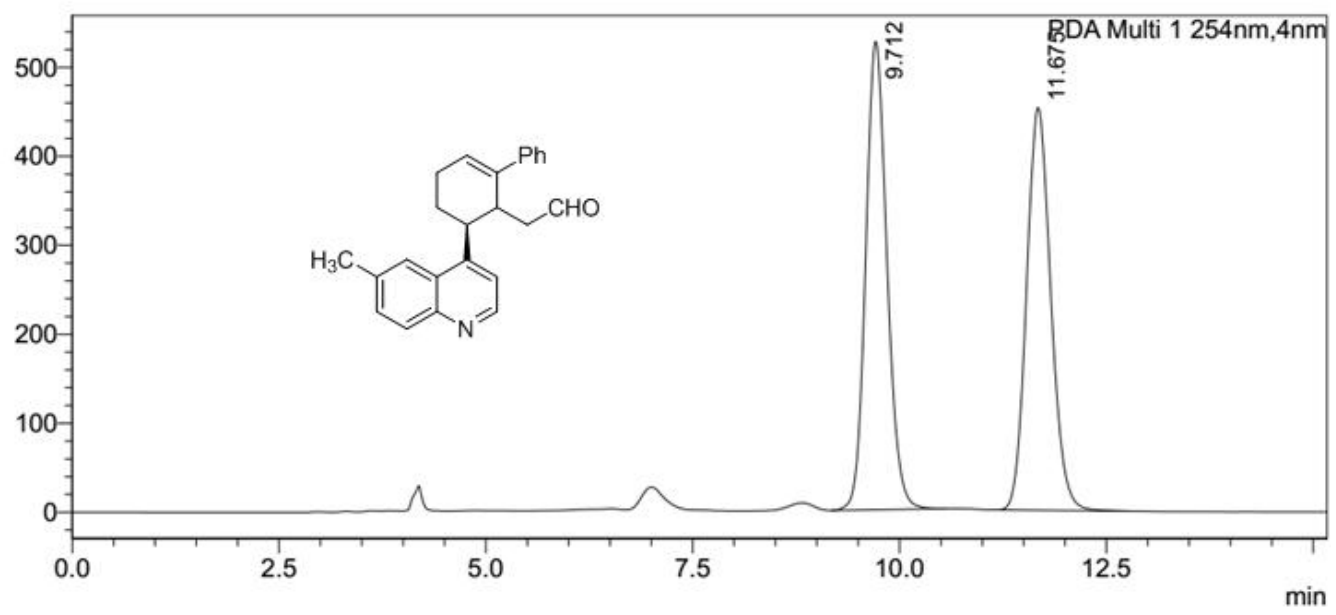

<Peak Table>

PDA Ch1 254nm

\begin{tabular}{|r|r|r|r|r|r|r|l|}
\hline Peak\# & Ret. Time & \multicolumn{1}{c|}{ Area } & Height & Conc. & Unit & Mark & \multicolumn{1}{c|}{ Name } \\
\hline 1 & 9.712 & 9421269 & 524455 & 50.487 & $\%$ & & RT:9.712 \\
\hline 2 & 11.675 & 9239643 & 451288 & 49.513 & $\%$ & & RT:11.675 \\
\hline Total & & 18660912 & 975743 & & & & \\
\hline
\end{tabular}

\section{$<$ Chromatogram >}

mAU

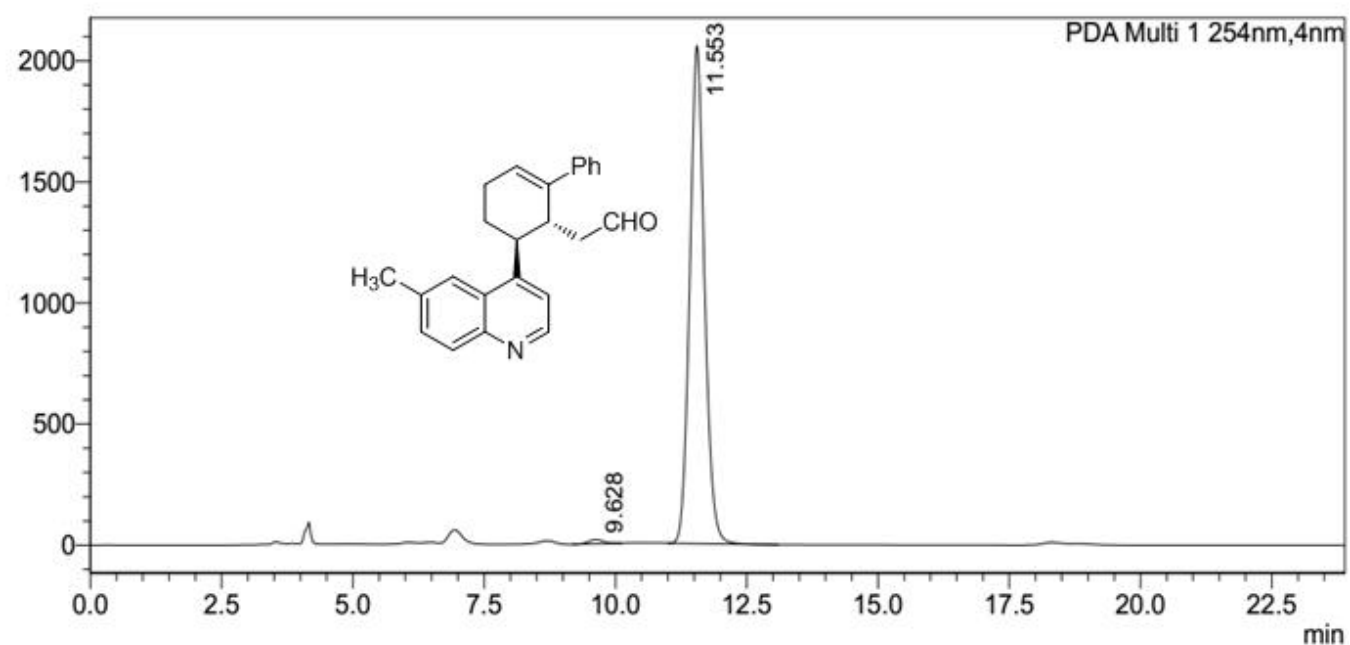

\section{<Peak Table>}

PDA Ch1 254nm

Peak\# Ret. Time

\begin{tabular}{|c|c|c|c|c|c|c|c|}
\hline Peak\# & Ret. Time & Area & Height & Conc. & Unit & Mark & Name \\
\hline 1 & 9.628 & 340725 & 17115 & 0.840 & $\%$ & & RT:9.628 \\
\hline 2 & 11.553 & 40237982 & 2038462 & 99.160 & $\%$ & & RT:11.553 \\
\hline Total & & 40578707 & 2055577 & & & & \\
\hline
\end{tabular}




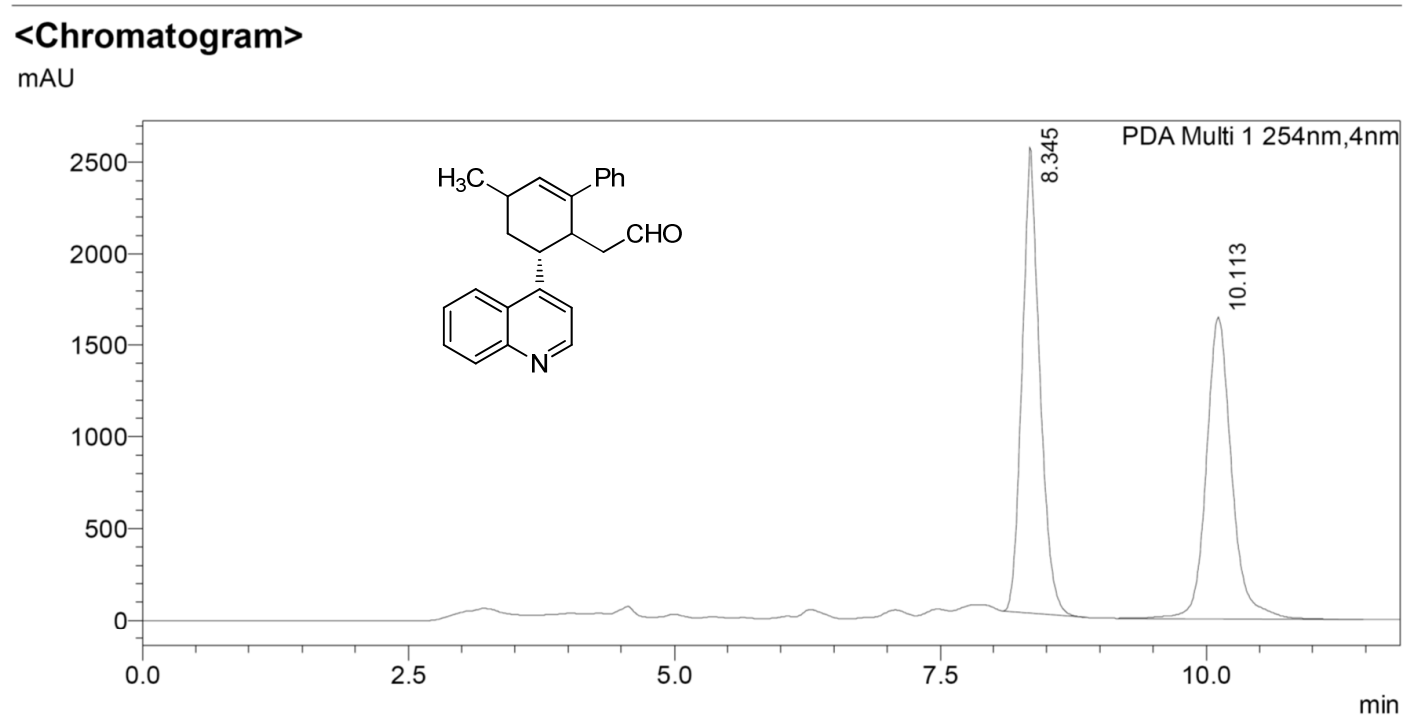

<Peak Table>

PDA Ch1 254nm

\begin{tabular}{|r|r|c|c|c|c|c|c|}
\hline Peak\# & Ret. Time & \multicolumn{1}{|c|}{ Area } & Height & Conc. & Unit & Mark & \multicolumn{2}{|c|}{ Name } \\
\hline 1 & 8.345 & 29261434 & 2539900 & 51.754 & $\%$ & & RT:8.345 \\
\hline 2 & 10.113 & 27278473 & 1639999 & 48.246 & $\%$ & & RT:10.113 \\
\hline Total & & 56539907 & 4179899 & & & & \\
\hline
\end{tabular}

\section{<Chromatogram>}

mAU

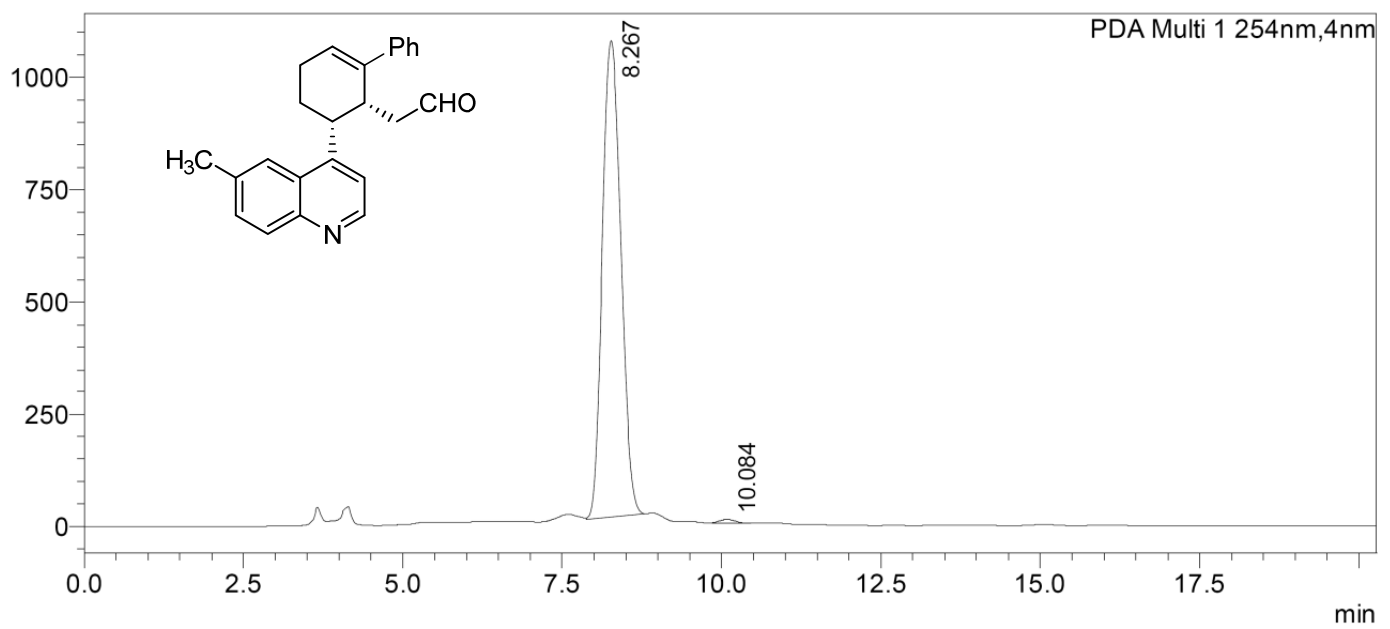

<Peak Table>

PDA Ch1 254nm

\begin{tabular}{|r|r|r|r|r|r|r|r|}
\hline Peak\# Ret. Time & \multicolumn{1}{|c|}{ Area } & Height & \multicolumn{1}{|c|}{ Conc. } & Unit & Mark & \multicolumn{2}{|c|}{ Name } \\
\hline 1 & 8.267 & 21497783 & 1055470 & 99.381 & $\%$ & M & RT:8.267 \\
\hline 2 & 10.084 & 133902 & 7755 & 0.619 & $\%$ & & RT:10.084 \\
\hline Total & & 21631685 & 1063224 & & & & \\
\hline
\end{tabular}


$<$ Chromatogram >

mAU

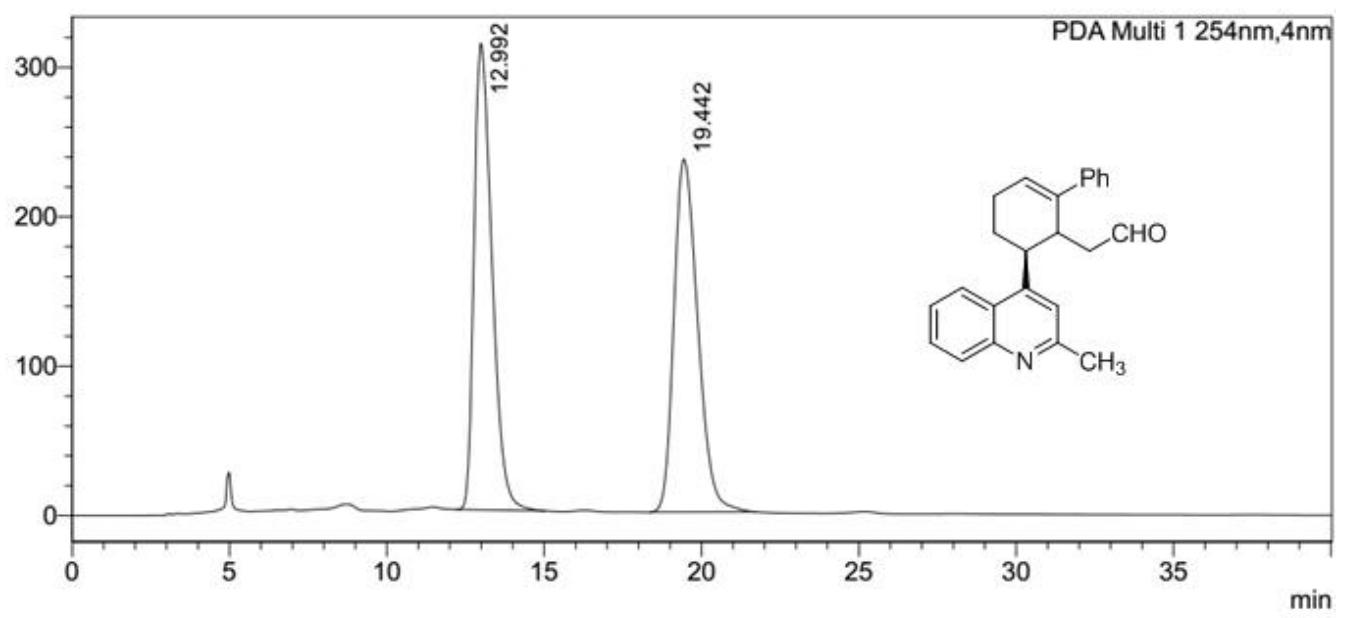

<Peak Table>

PDA Ch1 254nm

\begin{tabular}{|r|r|r|r|r|r|r|r|}
\hline Peak\# & Ret. Time & Area & Height & Conc. & Unit & Mark & \multicolumn{2}{|c|}{ Name } \\
\hline 1 & 12.992 & 12391658 & 311912 & 49.865 & $\%$ & & RT:12.992 \\
\hline 2 & 19.442 & 12458704 & 236141 & 50.135 & $\%$ & & RT:19.442 \\
\hline Total & & 24850362 & 548053 & & & & \\
\hline
\end{tabular}

<Chromatogram>

mAU

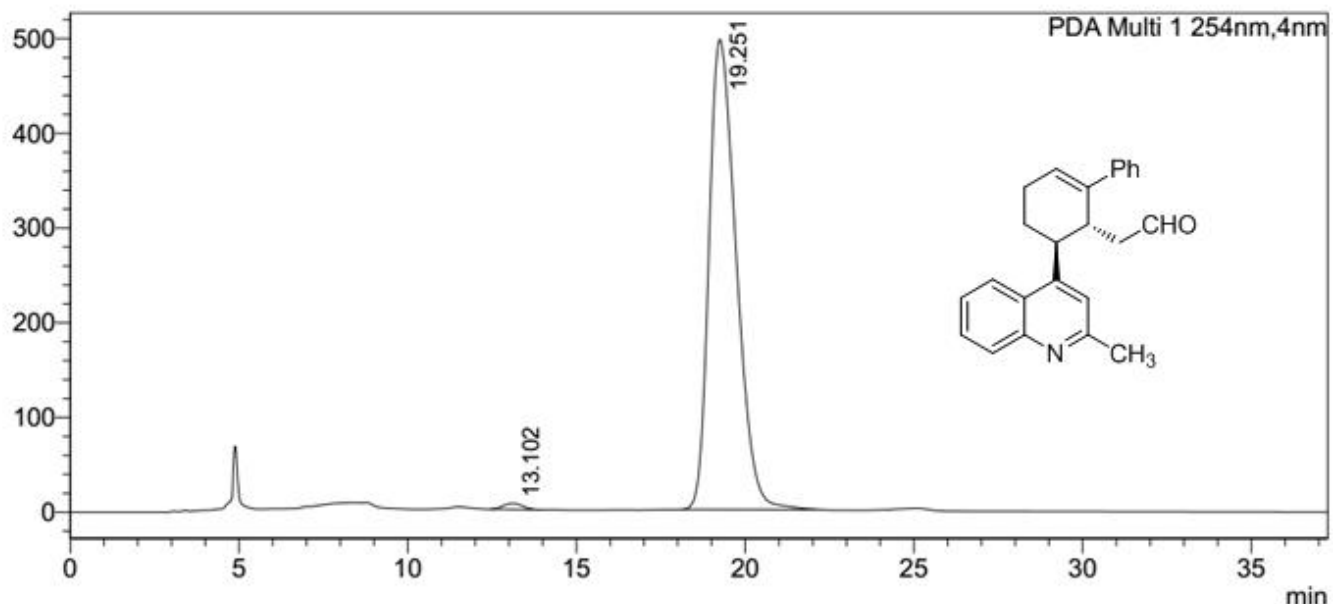

<Peak Table>

PDA Ch1 254nm Peak\# Ret. Time

\begin{tabular}{|r|r|r|r|r|r|r|r|}
\hline Peak\# & Ret. Time & \multicolumn{1}{|c|}{ Area } & \multicolumn{1}{c|}{ Height } & \multicolumn{1}{c|}{ Conc. } & Unit & Mark & \multicolumn{2}{|c|}{ Name } \\
\hline 1 & 13.102 & 253792 & 6333 & 0.902 & $\%$ & & RT: 13.102 \\
\hline 2 & 19.251 & 27888814 & 496205 & 99.098 & $\%$ & & RT:19.251 \\
\hline Total & & 28142606 & 502539 & & & & \\
\hline
\end{tabular}




\section{$<$ Chromatogram $>$}

mAU

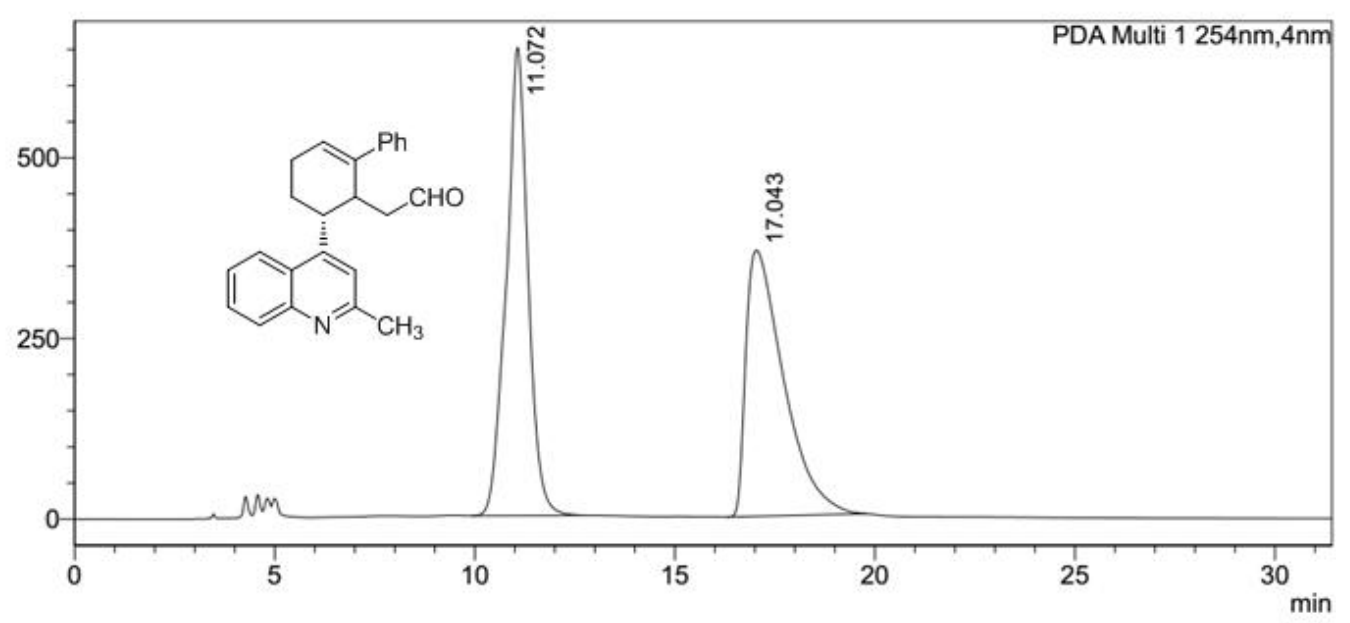

<Peak Table>

PDA Ch1 254nm

\begin{tabular}{|r|r|r|r|r|r|r|l|}
\hline Peak\# & Ret. Time & \multicolumn{1}{|c|}{ Area } & Height & Conc. & Unit & Mark & Name \\
\hline 1 & 11.072 & 24578851 & 646281 & 50.392 & $\%$ & & RT:11.072 \\
\hline 2 & 17.043 & 24196854 & 368156 & 49.608 & $\%$ & & RT:17.043 \\
\hline Total & & 48775705 & 1014437 & & & & \\
\hline
\end{tabular}

\section{<Chromatogram>}

mAU

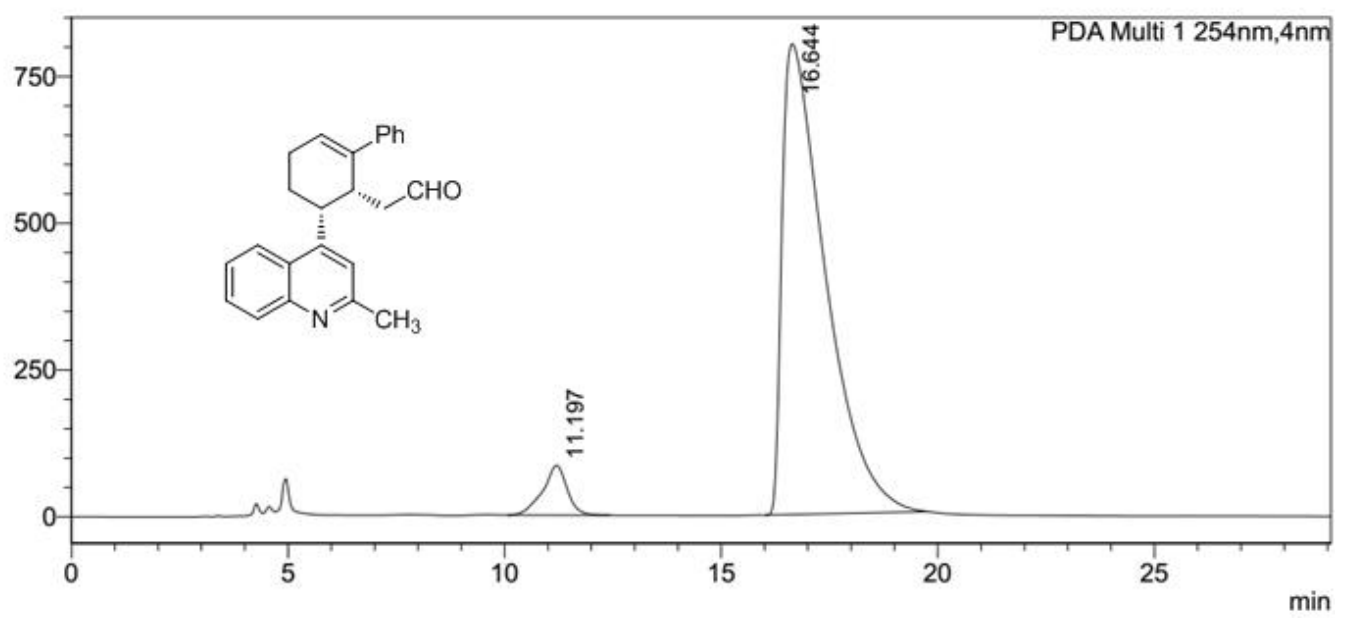

<Peak Table>

PDA Ch1 254nm

Peak\# Ret. Time

\begin{tabular}{|r|r|r|r|r|r|r|l|}
\hline Peak\# & Ret. Time & \multicolumn{1}{c|}{ Area } & \multicolumn{1}{c|}{ Height } & \multicolumn{1}{c|}{ Conc. } & Unit & Mark & \multicolumn{2}{|c|}{ Name } \\
\hline 1 & 11.197 & 3341376 & 84681 & 5.646 & $\%$ & & RT: 11.197 \\
\hline 2 & 16.644 & 55839128 & 800811 & 94.354 & $\%$ & & RT: 16.644 \\
\hline Total & & 59180504 & 885492 & & & & \\
\hline
\end{tabular}




\section{<Chromatogram>}

mAU

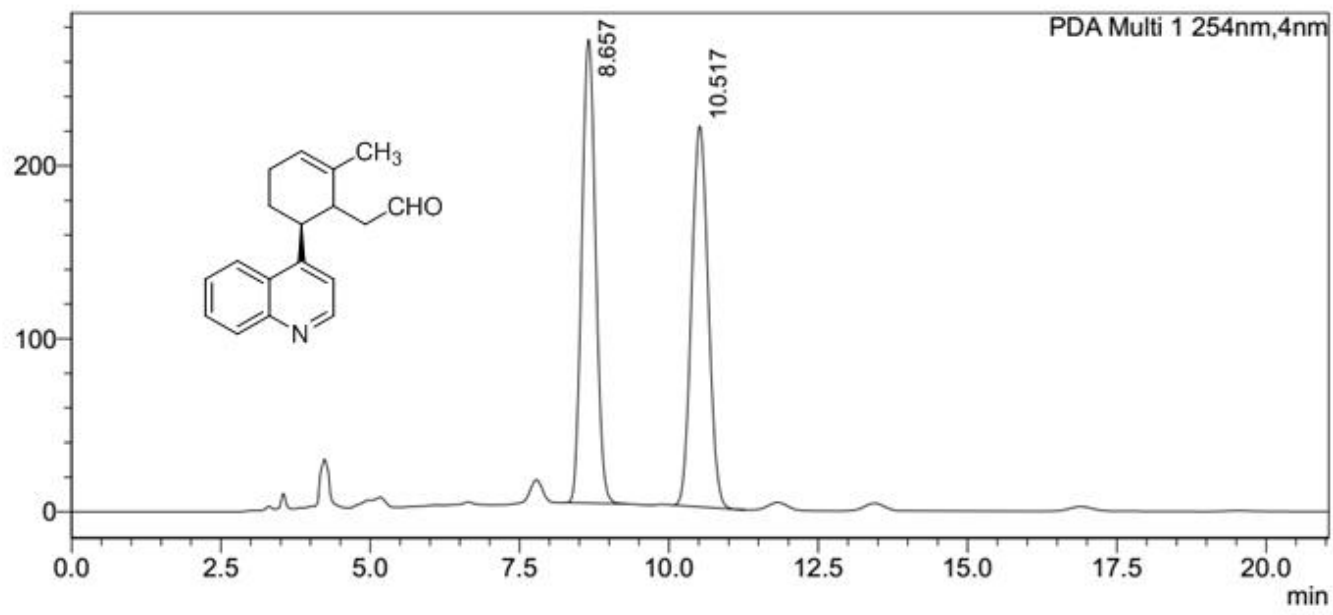

<Peak Table>

PDA Ch1 254nm

\begin{tabular}{|r|r|r|r|r|r|r|l|}
\hline Peak\# & Ret. Time & \multicolumn{1}{|c|}{ Area } & Height & Conc. & Unit & Mark & \multicolumn{1}{c|}{ Name } \\
\hline 1 & 8.657 & 4311604 & 267192 & 50.179 & $\%$ & & RT:8.657 \\
\hline 2 & 10.517 & 4280798 & 219598 & 49.821 & $\%$ & & RT:10.517 \\
\hline Total & & 8592402 & 486790 & & & & \\
\hline
\end{tabular}

\section{$<$ Chromatogram >}

mAU

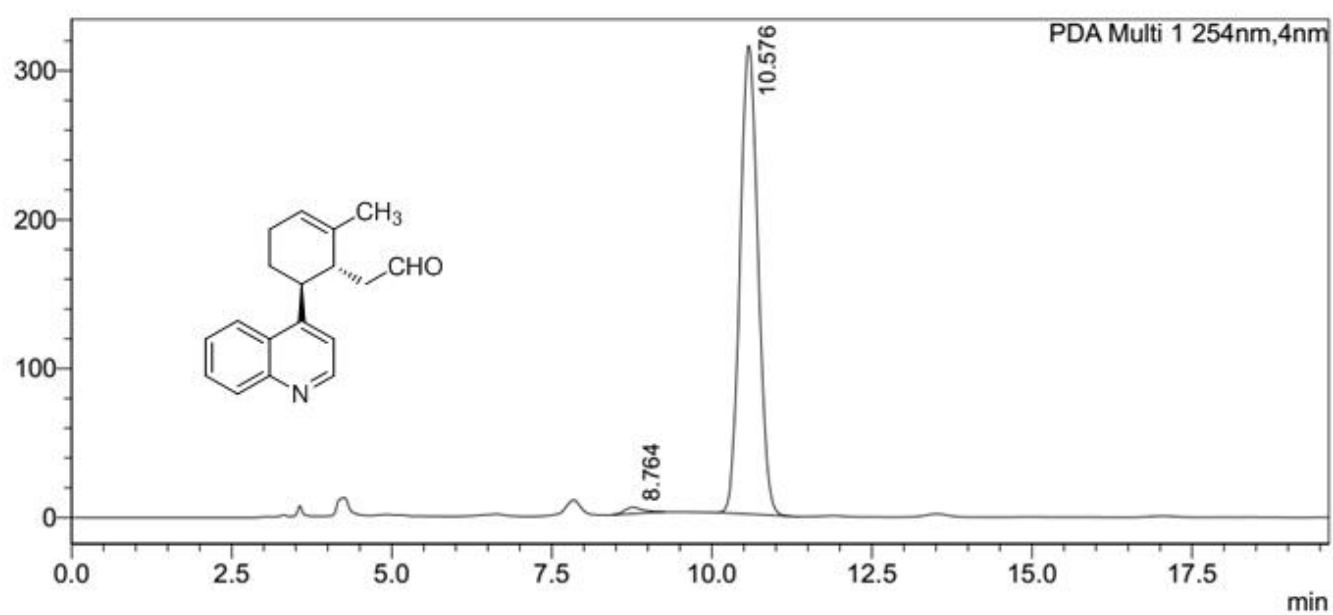

<Peak Table>

PDA Ch1 254nm

Peak\# Ret. Time

\begin{tabular}{|r|r|r|r|r|r|r|l|}
\hline Peak\# & Ret. Time & \multicolumn{1}{c|}{ Area } & Height & \multicolumn{1}{c|}{ Conc. } & Unit & Mark & \multicolumn{1}{c|}{ Name } \\
\hline 1 & 8.764 & 87484 & 4310 & 1.404 & $\%$ & & RT:8.764 \\
\hline 2 & 10.576 & 6143213 & 313641 & 98.596 & $\%$ & & RT:10.576 \\
\hline Total & & 6230697 & 317951 & & & & \\
\hline
\end{tabular}




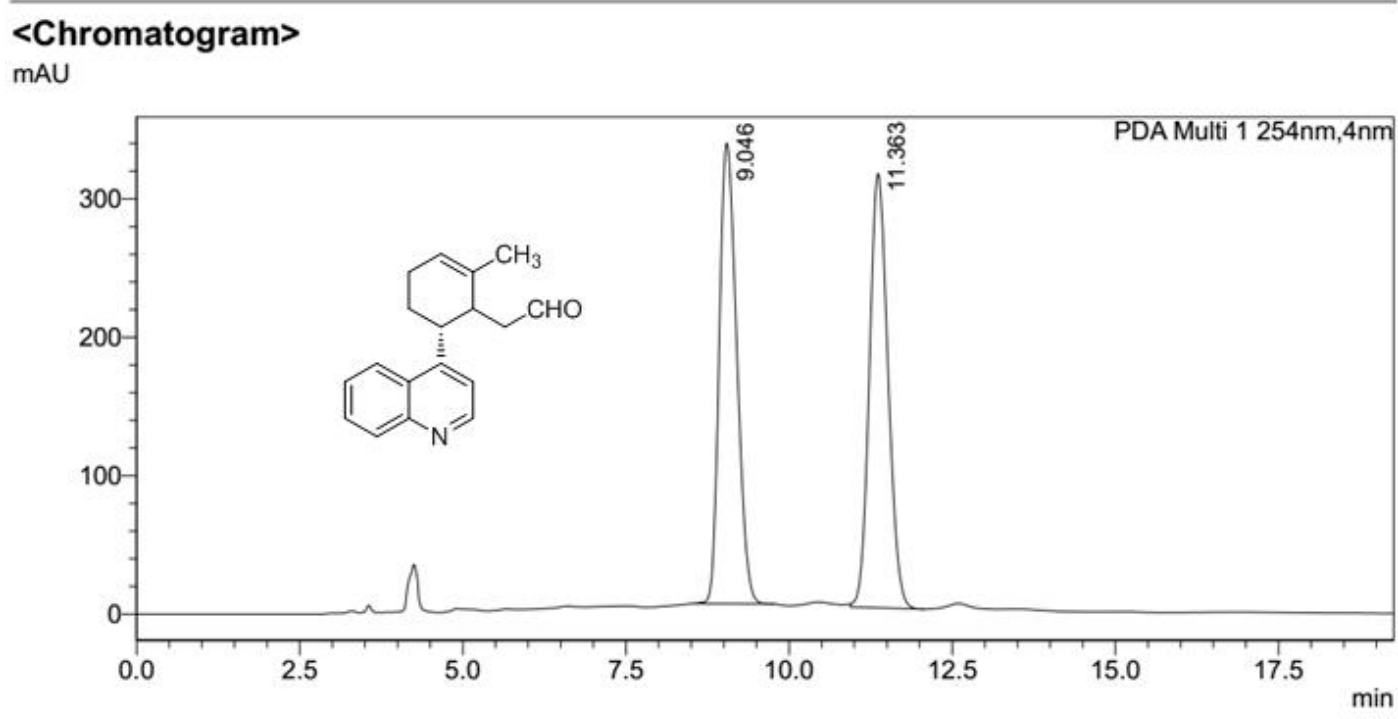

<Peak Table>

PDA Ch1 254nm

\begin{tabular}{|r|r|r|r|r|r|r|l|}
\hline Peak\# & Ret. Time & \multicolumn{1}{c|}{ Area } & Height & Conc. & Unit & Mark & Name \\
\hline 1 & 9.046 & 6196973 & 331831 & 49.912 & $\%$ & & RT:9.046 \\
\hline 2 & 11.363 & 6218844 & 311394 & 50.088 & $\%$ & V & RT:11.363 \\
\hline Total & & 12415818 & 643225 & & & & \\
\hline
\end{tabular}

\section{<Chromatogram>}

$\mathrm{mAU}$

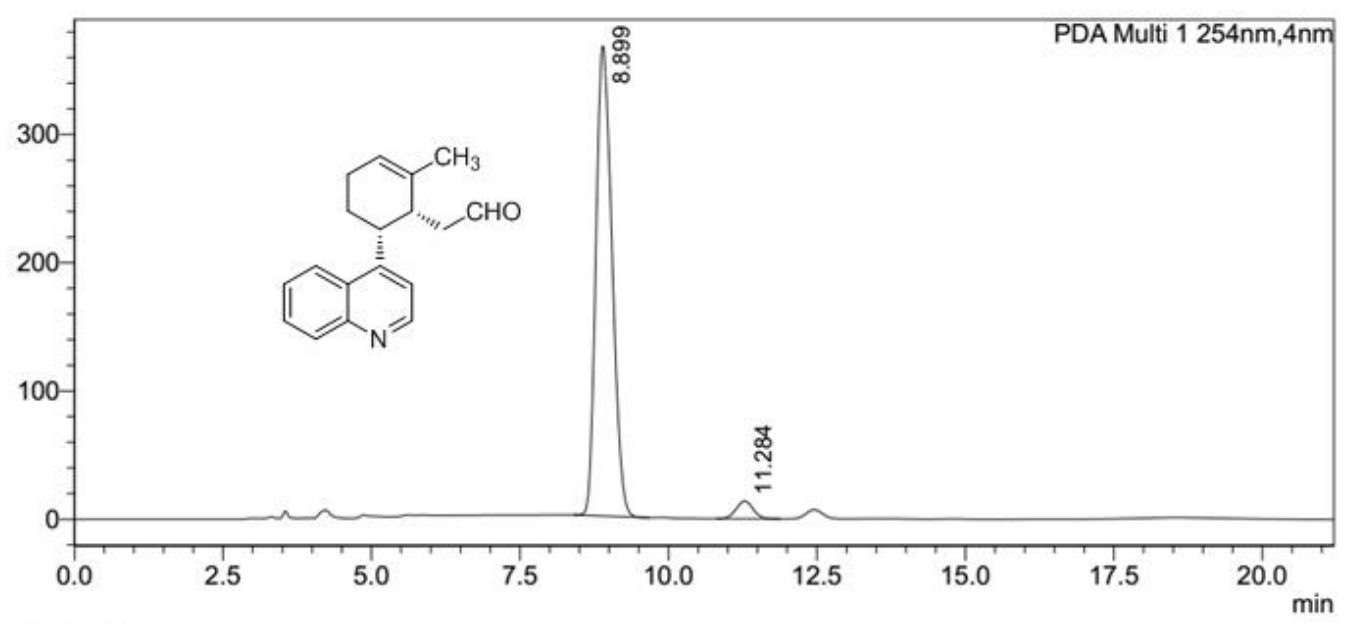

<Peak Table>

PDA Ch1 254nm

Peak\# Ret. Time

\begin{tabular}{|r|r|r|r|r|r|r|l|}
\hline Peak\# & Ret. Time & \multicolumn{1}{c|}{ Area } & Height & \multicolumn{1}{c|}{ Conc. } & Unit & Mark & \multicolumn{1}{c|}{ Name } \\
\hline 1 & 8.899 & 6927617 & 363738 & 96.163 & $\%$ & & RT:8.899 \\
\hline 2 & 11.284 & 276385 & 13829 & 3.837 & $\%$ & & RT:11.284 \\
\hline Total & & 7204002 & 377567 & & & & \\
\hline
\end{tabular}


$<$ Chromatogram>

mAU

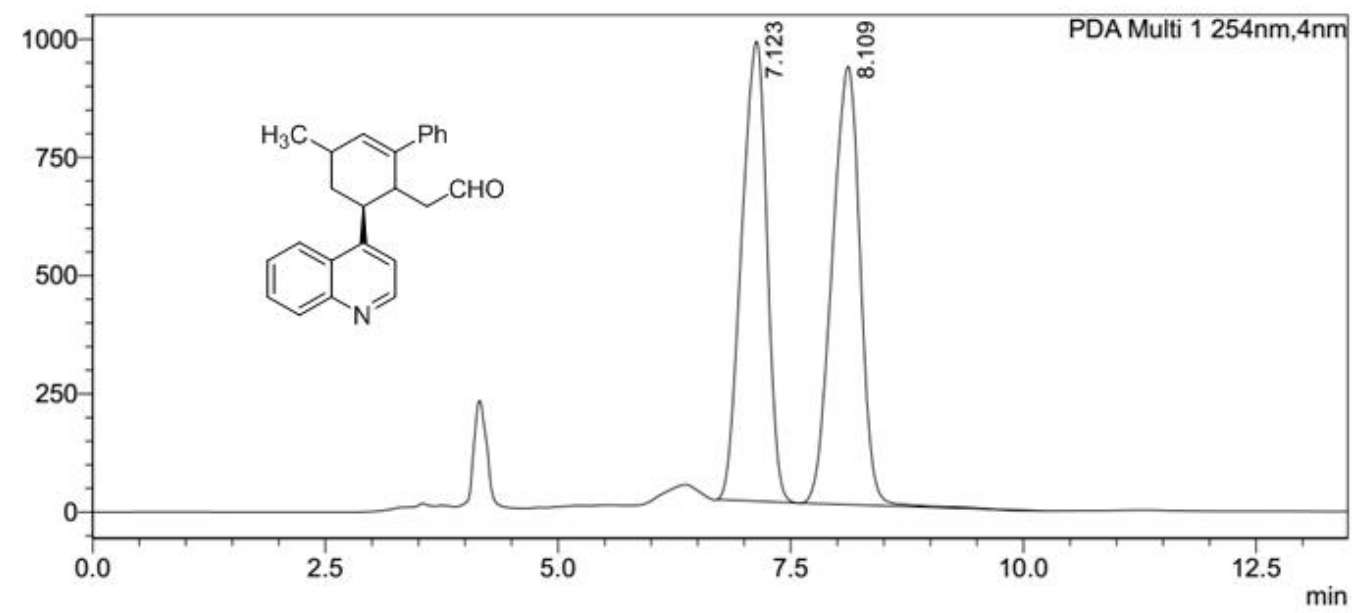

<Peak Table>

PDA Ch1 254nm

\begin{tabular}{|r|r|r|r|r|r|r|l|}
\hline Peak\# & Ret. Time & \multicolumn{1}{c|}{ Area } & Height & \multicolumn{1}{c|}{ Conc. } & Unit & Mark & Name \\
\hline 1 & 7.123 & 18311707 & 957461 & 48.494 & $\%$ & & RT:7.123 \\
\hline 2 & 8.109 & 19448959 & 913732 & 51.506 & $\%$ & & RT:8.109 \\
\hline Total & & 37760666 & 1871193 & & & & \\
\hline
\end{tabular}

<Chromatogram>

MAU

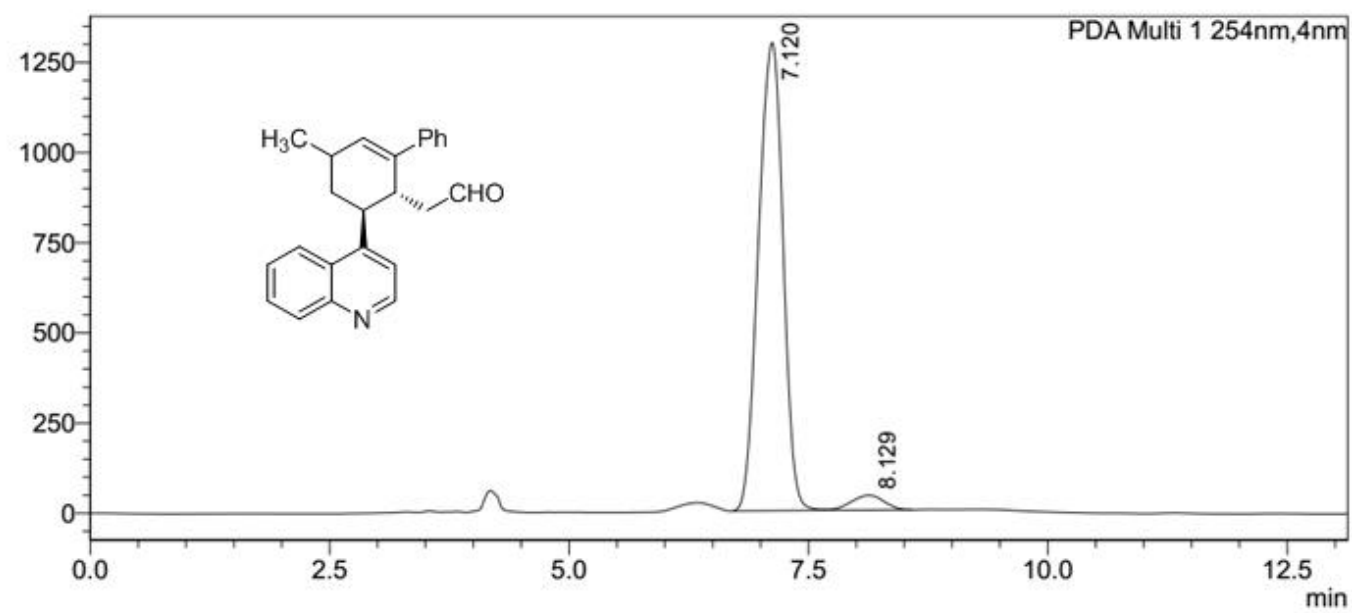

<Peak Table>

PDA Ch1 254nm

\begin{tabular}{rr|} 
Peak\# Ret. Time \\
\hline 1.120
\end{tabular}

\begin{tabular}{|r|r|r|r|r|r|r|r|}
\hline Peak\# & Ret. Time & \multicolumn{1}{c|}{ Area } & \multicolumn{1}{c|}{ Height } & \multicolumn{1}{c|}{ Conc. } & Unit & Mark & \multicolumn{2}{|c|}{ Name } \\
\hline 1 & 7.120 & 23234554 & 1294412 & $95.989 \%$ & & RT:7.120 \\
\hline 2 & 8.129 & 970944 & 40798 & $4.011 \%$ & V & RT:8.129 & \\
\hline Total & & 24205498 & 1335210 & & & & \\
\hline
\end{tabular}




\section{<Chromatogram>}

mAU

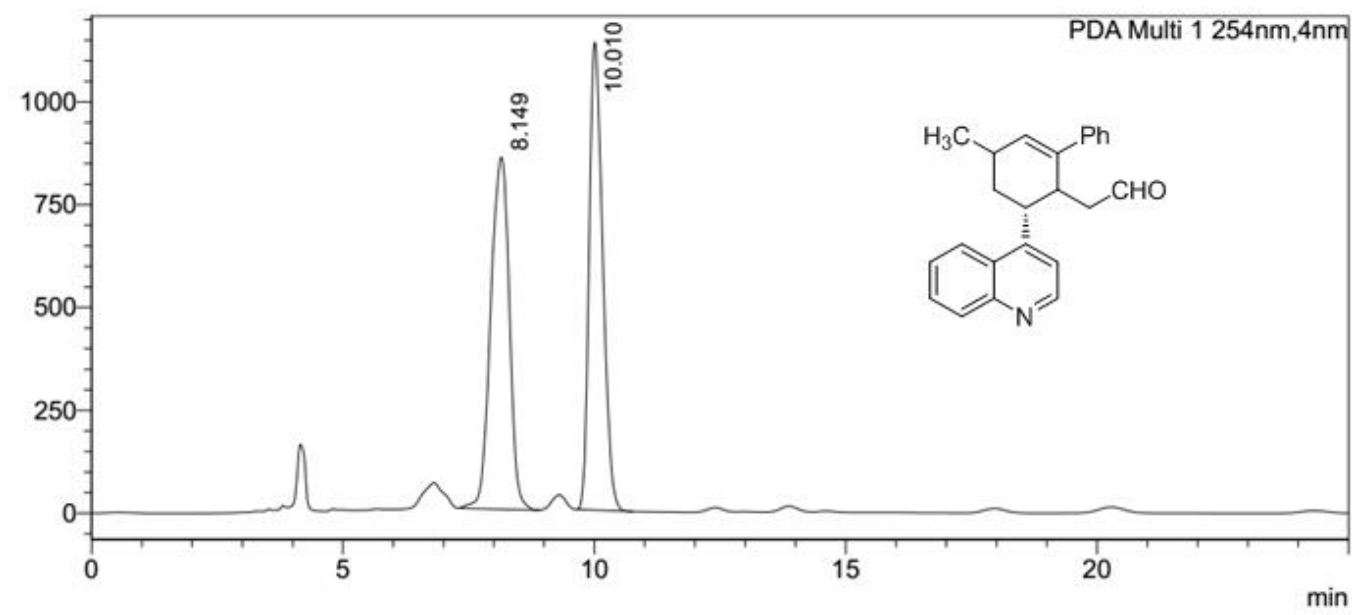

<Peak Table>

PDA Ch1 254nm

\begin{tabular}{|r|r|r|r|r|r|r|l|}
\hline Peak\# & Ret. Time & Area & Height & Conc. & Unit & Mark & \multicolumn{2}{|c|}{ Name } \\
\hline 1 & 8.149 & 22112948 & 854717 & 50.958 & $\%$ & & RT:8.149 \\
\hline 2 & 10.010 & 21281107 & 1134276 & 49.042 & $\%$ & & RT: 10.010 \\
\hline Total & & 43394055 & 1988993 & & & & \\
\hline
\end{tabular}

$<$ Chromatogram >

mAU

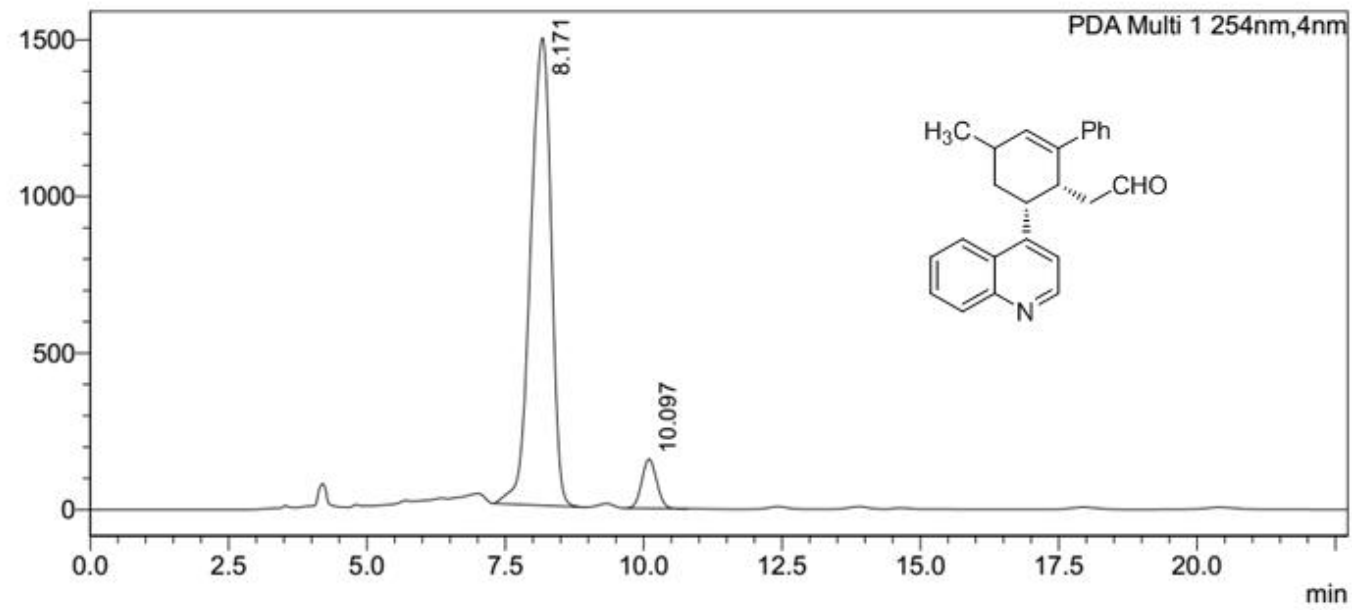

<Peak Table>

PDA Ch1 254nm

Peak\# Ret. Time

\begin{tabular}{|r|r|r|r|r|r|r|l|}
\hline Peak\# & Ret. Time & \multicolumn{1}{c|}{ Area } & \multicolumn{1}{c|}{ Height } & \multicolumn{1}{c|}{ Conc. } & Unit & Mark & \multicolumn{1}{c|}{ Name } \\
\hline 1 & 8.171 & 38685158 & 1489838 & 92.949 & $\%$ & & RT:8.171 \\
\hline 2 & 10.097 & 2934525 & 157018 & 7.051 & $\%$ & & RT:10.097 \\
\hline Total & & 41619683 & 1646856 & & & & \\
\hline
\end{tabular}


$<$ Chromatogram>

mAU

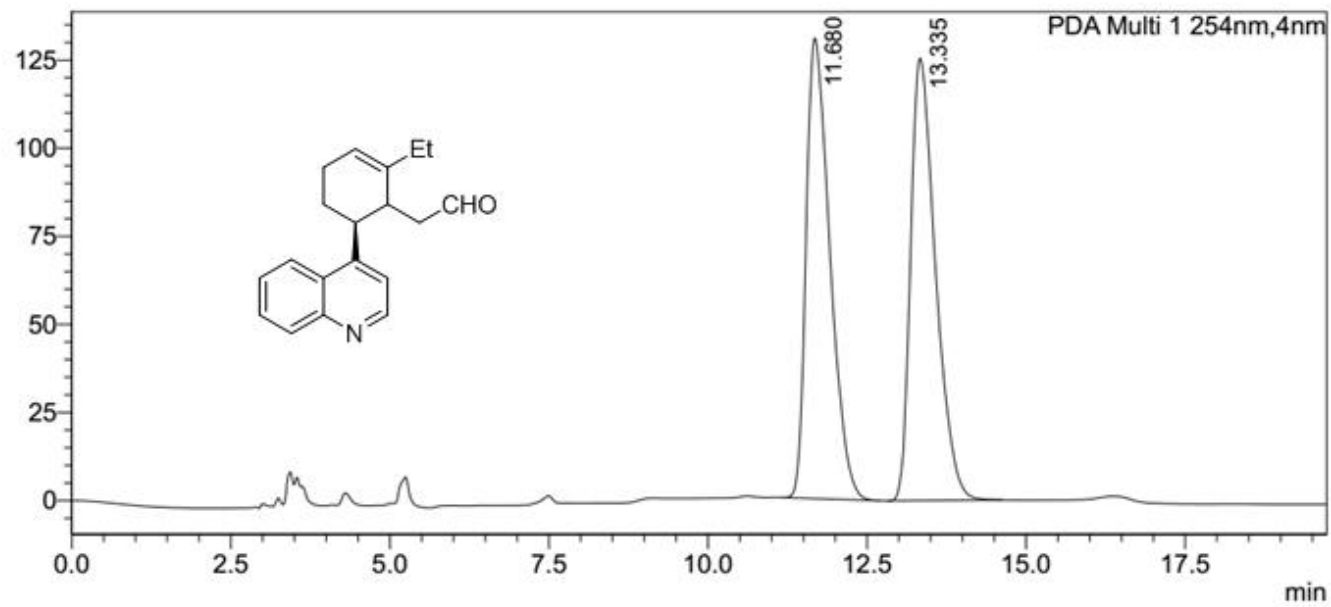

<Peak Table>

PDA Ch1 254nm

\begin{tabular}{|r|r|r|r|r|r|r|r|}
\hline Peak\# & Ret. Time & Area & Height & Conc. & Unit & Mark & \multicolumn{2}{|c|}{ Name } \\
\hline 1 & 11.680 & 3395958 & 130706 & 49.804 & $\%$ & & RT: 11.680 \\
\hline 2 & 13.335 & 3422644 & 125528 & 50.196 & $\%$ & & RT: 13.335 \\
\hline Total & & 6818602 & 256234 & & & & \\
\hline
\end{tabular}

\section{$<$ Chromatogram >}

mAU

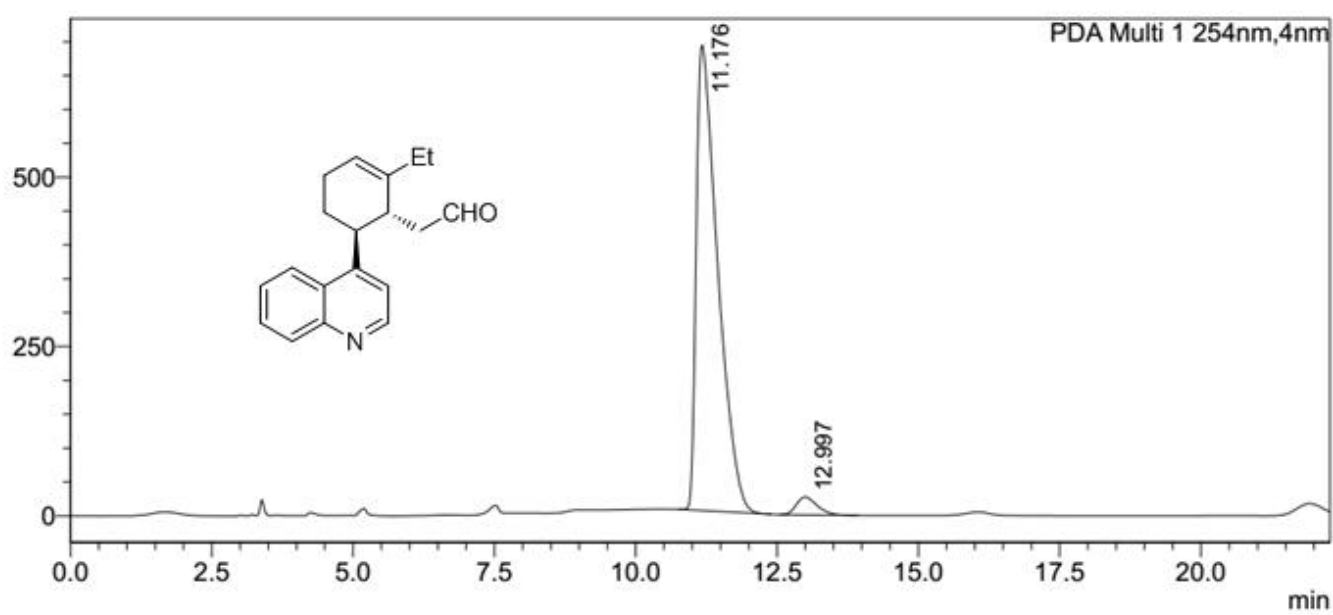

<Peak Table>

PDA Ch1 254nm Peak\# Ret. Time

\begin{tabular}{|r|r|r|r|r|r|r|r|}
\hline Peak\# & Ret. Time & \multicolumn{1}{c|}{ Area } & \multicolumn{1}{c|}{ Height } & \multicolumn{1}{c|}{ Conc. } & Unit & Mark & \multicolumn{2}{|c|}{ Name } \\
\hline 1 & 11.176 & 18104173 & 687246 & 96.408 & $\%$ & & RT: 11.176 \\
\hline 2 & 12.997 & 674447 & 26344 & 3.592 & $\%$ & & RT:12.997 \\
\hline Total & & 18778620 & 713590 & & & & \\
\hline
\end{tabular}


<Chromatogram>

MAU

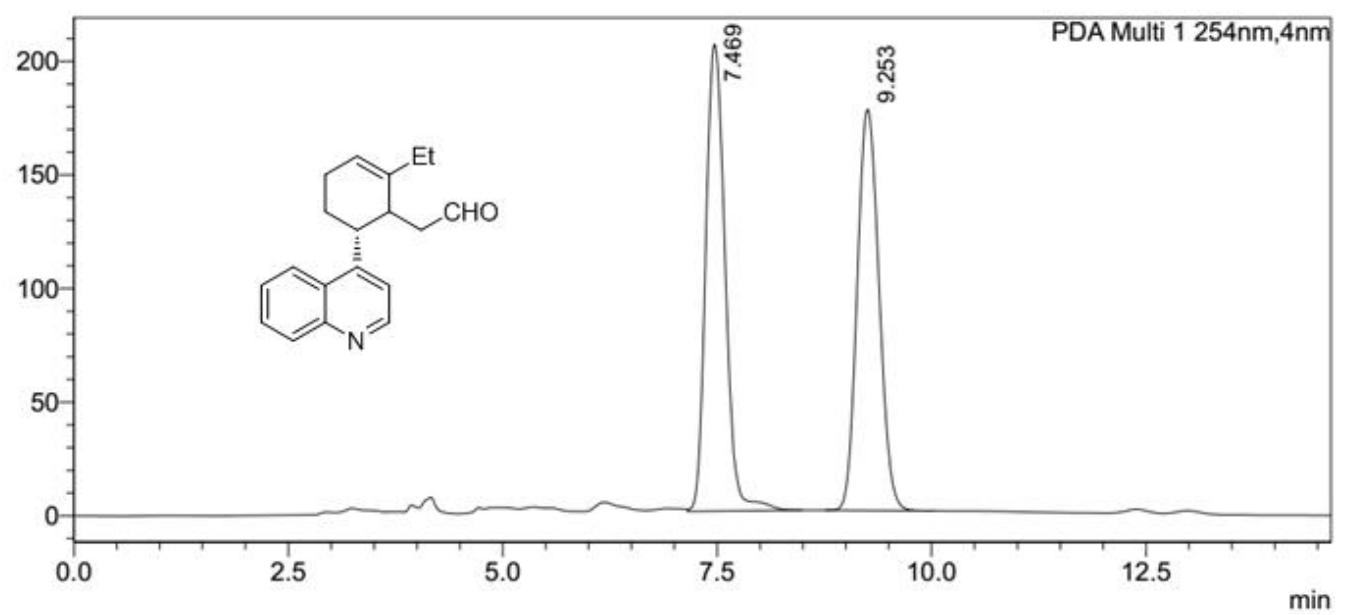

<Peak Table>

PDA Ch1 254nm

\begin{tabular}{|r|r|r|r|r|r|r|l|}
\hline Peak\# & Ret. Time & Area & Height & Conc. & Unit & Mark & Name \\
\hline 1 & 7.469 & 3227206 & 205526 & 50.836 & $\%$ & V & RT:7.469 \\
\hline 2 & 9.253 & 3121005 & 176448 & 49.164 & $\%$ & & RT:9.253 \\
\hline Total & & 6348211 & 381974 & & & & \\
\hline
\end{tabular}

$<$ Chromatogram>

mAU

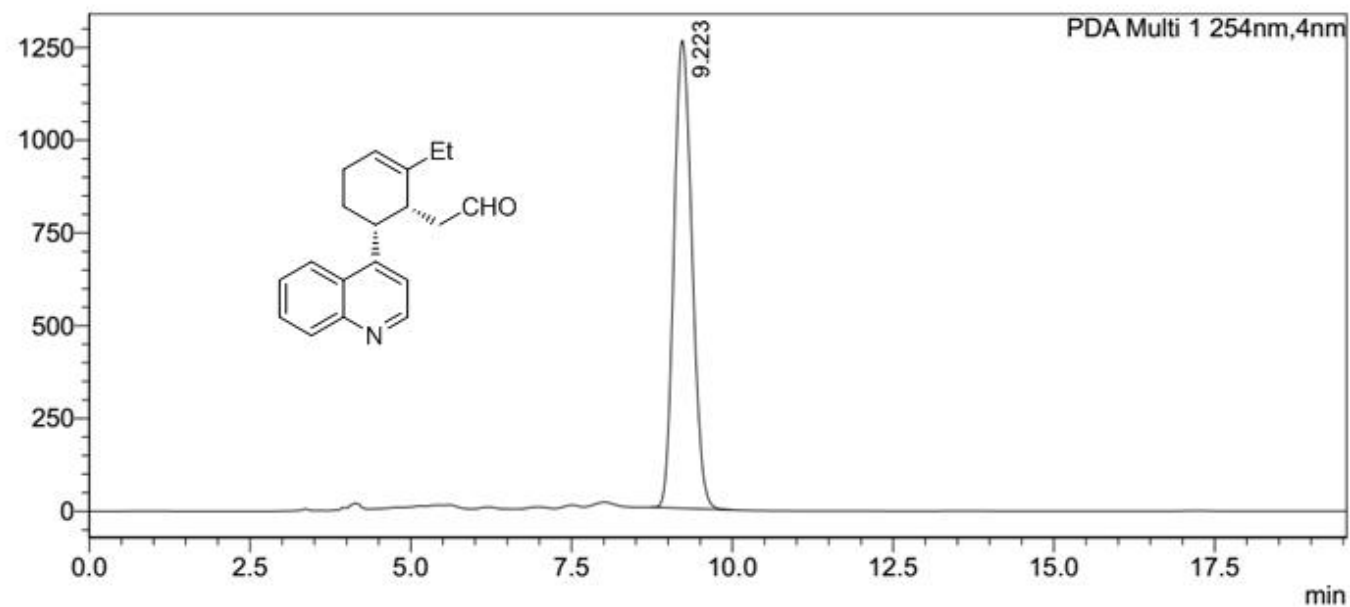

<Peak Table>

PDA Ch1 254nm

\begin{tabular}{|c|c|c|c|c|c|c|c|}
\hline Peak\# & Ret. Time & Area & Height & Conc. & Unit & Mark & Name \\
\hline 1 & 9.223 & 24693903 & 1261364 & 100.000 & $\%$ & & RT:9.223 \\
\hline Tota & & 24693903 & 1261364 & & & & \\
\hline
\end{tabular}




\section{<Chromatogram>}

mAU

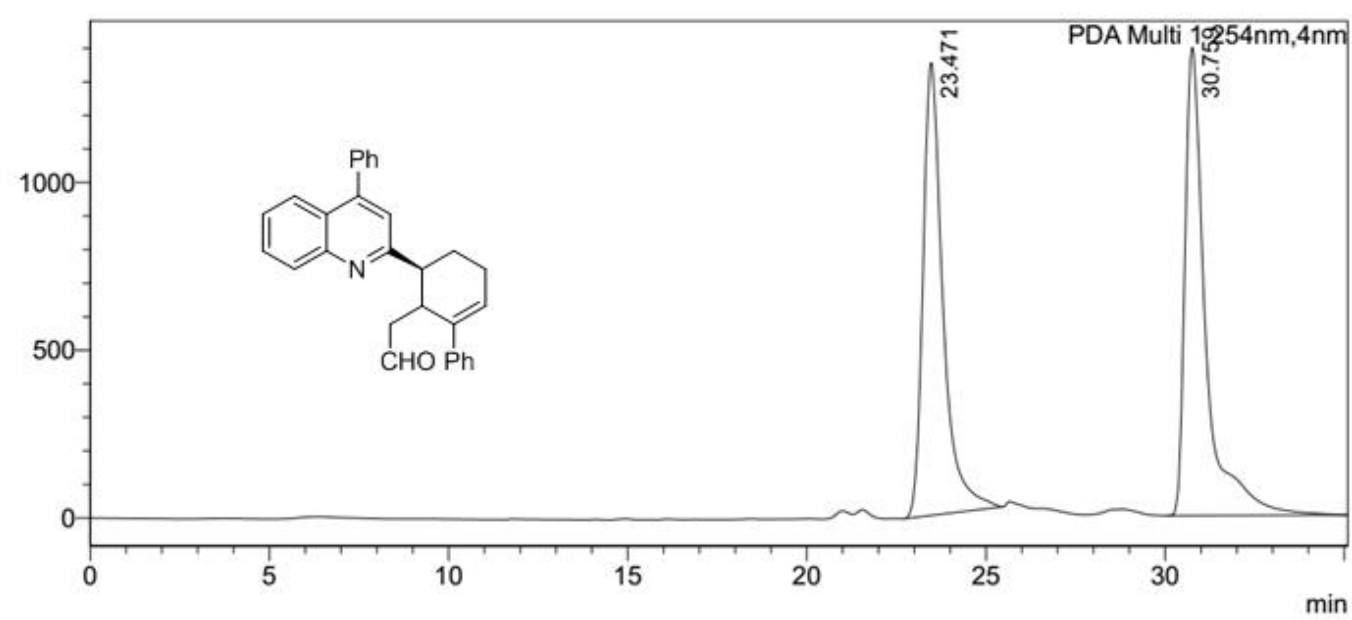

<Peak Table>

PDA Ch1 254nm

\begin{tabular}{|c|c|c|c|c|c|c|c|}
\hline Peak\# & et. Time & Area & Height & Conc. & Unit & Mark & Name \\
\hline 1 & 23.471 & 52313491 & 1349795 & 48.170 & $\%$ & & RT:23.471 \\
\hline 2 & 30.759 & 56287601 & 1395728 & 51.830 & $\%$ & & RT:30.759 \\
\hline Total & & 108601092 & 2745523 & & & & \\
\hline
\end{tabular}

\section{<Chromatogram>}

mAU

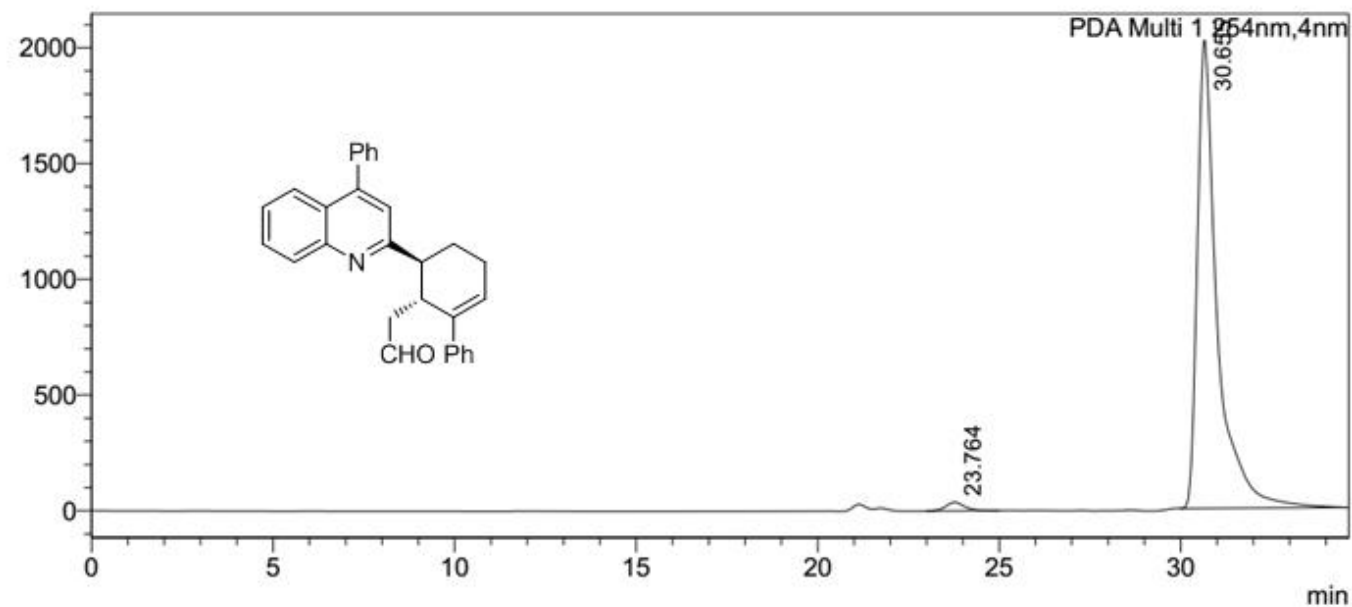

<Peak Table>

PDA Ch1 254nm

\begin{tabular}{|r|r|}
\hline Peak\# Ret. Time \\
\hline 1 & 23.764 \\
\hline
\end{tabular}

\begin{tabular}{|r|r|}
\hline 1 & 23.76 \\
\hline 2 & 30.655 \\
\hline
\end{tabular}

\begin{tabular}{|r|r|r|r|r|}
\hline \multicolumn{1}{|c|}{ Area } & Height & Conc. & Unit & Mark \\
\hline 1400290 & 37711 & 1.771 & $\%$ & \\
\hline 77662980 & 2022694 & 98.229 & $\%$ & V \\
\hline 79063270 & 2060405 & & & \\
\hline
\end{tabular}

RT:23.764

RT:30.655

Name 


\section{<Chromatogram>}

mAU

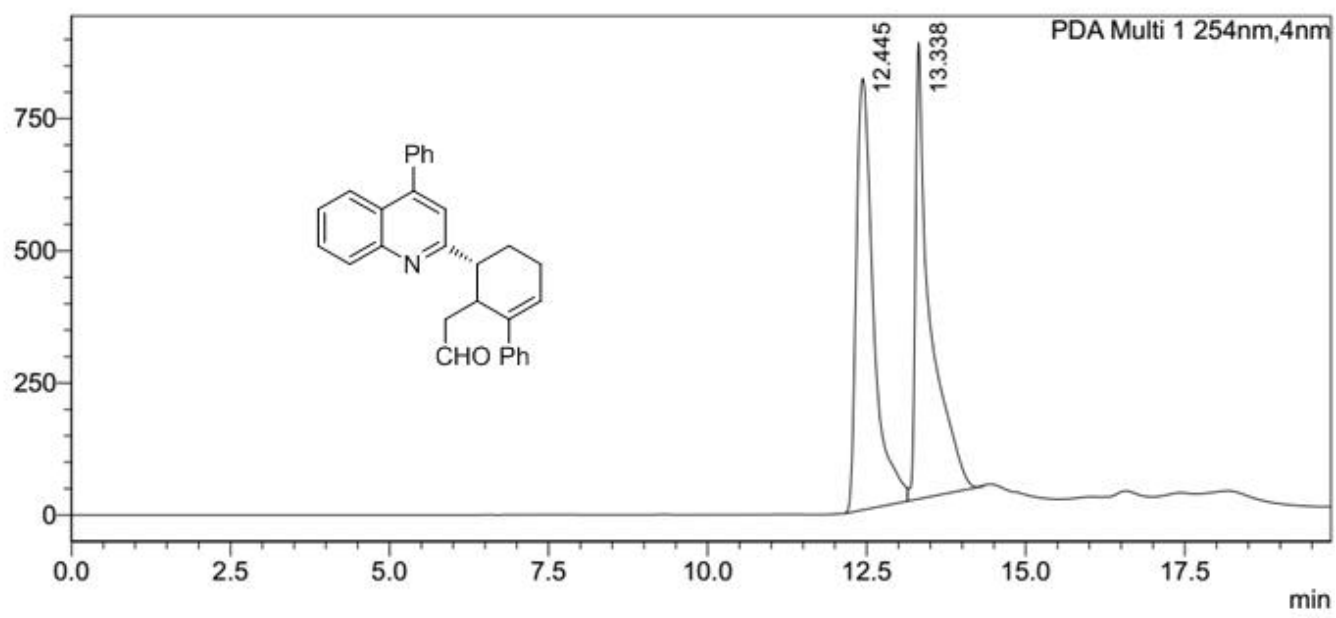

<Peak Table>

PDA Ch1 254nm

\begin{tabular}{|r|r|r|r|r|r|r|r|}
\hline Peak\# & Ret. Time & \multicolumn{1}{c|}{ Area } & Height & Conc. & Unit & Mark & Name \\
\hline 1 & 12.445 & 15403385 & 802921 & 52.485 & $\%$ & & RT:12.445 \\
\hline 2 & 13.338 & 13944513 & 784353 & 47.515 & $\%$ & V & RT:13.338 \\
\hline Total & & 29347898 & 1587274 & & & & \\
\hline
\end{tabular}

<Chromatogram>

mAU

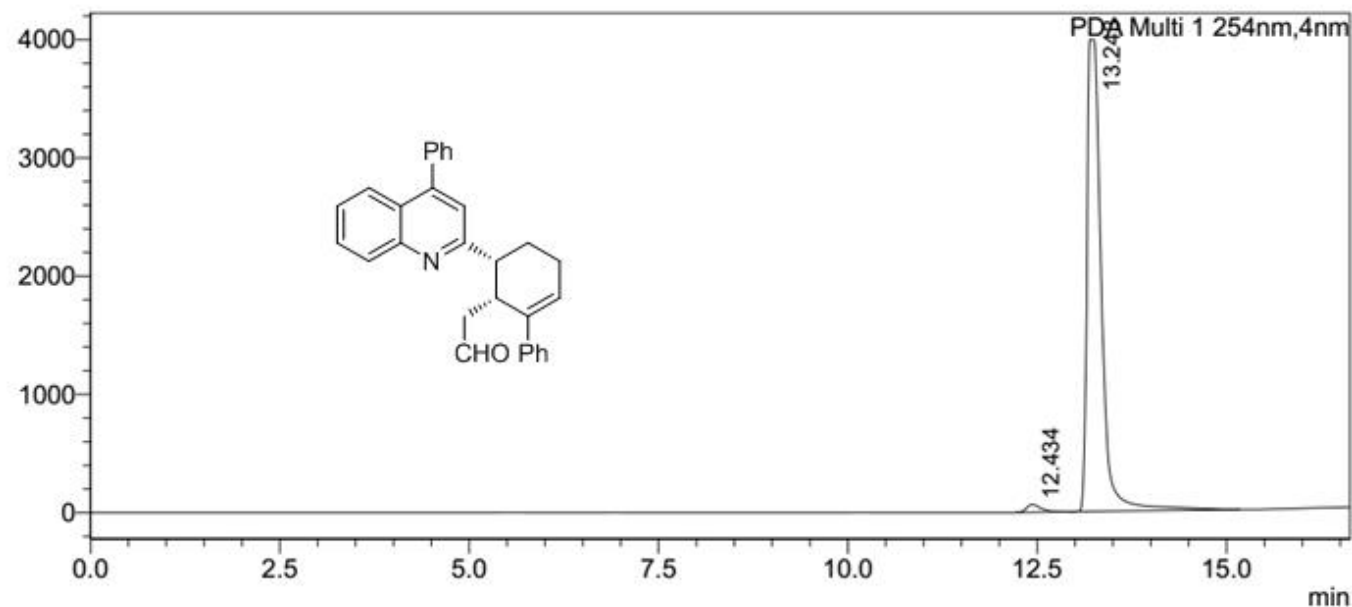

<Peak Table>

PDA Ch1 254nm

Peak\# Ret. Time

\begin{tabular}{|r|r|r|r|r|r|r|r|}
\hline Peak\# & Ret. Time & \multicolumn{1}{|c|}{ Area } & \multicolumn{1}{c|}{ Height } & \multicolumn{1}{|c|}{ Conc. } & Unit & Mark & \multicolumn{2}{|c|}{ Name } \\
\hline 1 & 12.434 & 900935 & 65909 & 1.685 & $\%$ & & RT:12.434 \\
\hline 2 & 13.243 & 52577478 & 3990009 & 98.315 & $\%$ & & RT:13.243 \\
\hline Total & & 53478413 & 4055918 & & & & \\
\hline
\end{tabular}

